\title{
A Methodology for Validating Building Energy Analysis Simulations
}

\section{Technical Report} NREL/TP-550-42059

April 2008

\author{
R. Judkoff, D. Wortman, B. O’Doherty, \\ and J. Burch \\ National Renewable Energy Laboratory
}

This work was performed during the early 1980 s and the report was written in 1983 but not published. It was, however, distributed informally to some experts in the field and was referenced as SERI/TR-254-1508. It was recently prepared for publication, but the information has not been updated.

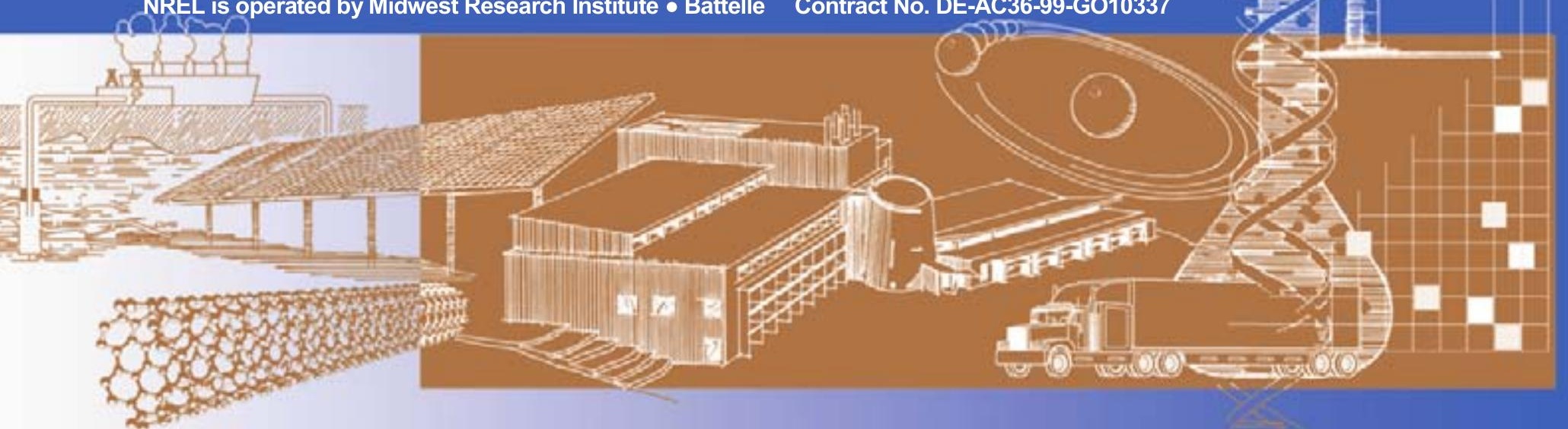




\section{A Methodology for Validating Building Energy Analysis Simulations}

R. Judkoff, D. Wortman, B. O'Doherty, and J. Burch

National Renewable Energy Laboratory

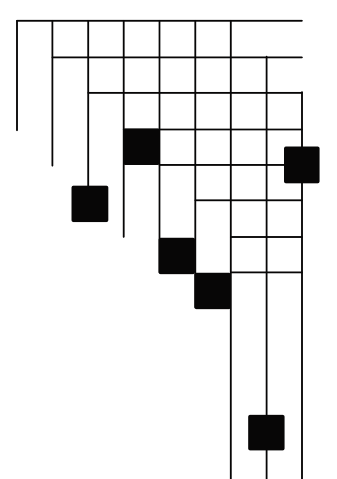

Prepared under Task No. 54004000 


\section{NOTICE}

This report was prepared as an account of work sponsored by an agency of the United States government. Neither the United States government nor any agency thereof, nor any of their employees, makes any warranty, express or implied, or assumes any legal liability or responsibility for the accuracy, completeness, or usefulness of any information, apparatus, product, or process disclosed, or represents that its use would not infringe privately owned rights. Reference herein to any specific commercial product, process, or service by trade name, trademark, manufacturer, or otherwise does not necessarily constitute or imply its endorsement, recommendation, or favoring by the United States government or any agency thereof. The views and opinions of authors expressed herein do not necessarily state or reflect those of the United States government or any agency thereof.

Available electronically at http://www.osti.gov/bridge

Available for a processing fee to U.S. Department of Energy and its contractors, in paper, from:

U.S. Department of Energy

Office of Scientific and Technical Information

P.O. Box 62

Oak Ridge, TN 37831-0062

phone: 865.576 .8401

fax: 865.576 .5728

email: mailto:reports@adonis.osti.gov

Available for sale to the public, in paper, from:

U.S. Department of Commerce

National Technical Information Service

5285 Port Royal Road

Springfield, VA 22161

phone: 800.553.6847

fax: 703.605.6900

email: orders@ntis.fedworld.gov

online ordering: http://www.ntis.gov/ordering.htm 


\section{Foreword}

This is a publication of work that was almost completed in August 1983. Final publication was never completed at that time because of funding issues. There was, however, a limited distribution of the final draft to leading experts in the field, and the report has been referenced in a number of documents nationally and internationally. Since that time great strides have been made in computer hardware. It is now possible for a building design practitioner to run a full-blown simulation of building energy performance on a laptop computer, and there are literally hundreds of such computer programs throughout the world. Thus, there is renewed interest in the theory of how to validate building energy simulation programs. We have therefore cleaned up the few cosmetic edits that remained in the previous final draft and formally published it as NREL/TP-550-42059 (originally SERI/TR-2541508). Although the simulation programs referred to in this report have long since been replaced by many subsequent versions of software, the underlying theory of how to validate, diagnose, and design good validation experiments has remained substantially unchanged since we first proposed this methodology. 


\section{Executive Summary}

\section{Objective}

To develop a validation methodology for building energy analysis simulations (BEAS), collect high-quality, unambiguous empirical data for validation, and apply the validation methodology to the DOE-2.1, BLAST-2MRT, BLAST-3.0, DEROB-3, DEROB-4, and SUNCAT 2.4 computer programs.

\section{Discussion}

This report covers background information, literature survey, validation methodology, comparative studies, analytical verification, empirical validation, comparative evaluation of codes, and conclusions. Section 1.0 establishes the historical context in which the Solar Energy Research Institute (SERI) studies evolved. The history of computerized building energy analysis is traced and the case is made that earlier methods do not contain algorithms that can accurately determine all heat flow quantities, especially for natural heating and cooling applications. These programs, though versatile for conventional buildings, are highly questionable for analyzing innovative design options. Newer state-of-the-art programs, such as DOE-2.I, BLAST-3.0, DEROB-4, and SUNCAT-2.4, have not yet been sufficiently validated over a wide enough range of parameters to be used with confidence. Researchers, representatives of the building industry, and several government-sponsored planning groups have expressed the need for a systematic approach to the validation issue.

Section 2.0 reviews a sampling of the literature on the validation of building energy analysis simulations, which shows that previous validation studies left four areas needing further investigation:

- Validation with empirical data from full-scale buildings: In previous studies there generally have not been sufficient data to understand observed differences between calculated and empirical results. Little effort has been directed toward performing follow-up experiments and reducing ambiguities in the data. Furthermore, data are lacking for buildings using natural environmental control systems.

- Validation with empirical data from test cells: Many validation studies have been done using single-zone test cells; however, few investigators have confidence in the extrapolation from single- to multi-zone predictions. Nevertheless, there are few or no data from multi-zone test cells or multi-zone unoccupied buildings.

- Analytical verification and code-to-code comparisons: Most comparative software studies have been done on conventional buildings. Studies on buildings that use natural environmental control systems are lacking. Additionally, previous comparative validation studies have not exploited the combined analytical and comparative approach.

- Validation methodology: Although validation studies have been performed, no systematic validation methodology has been developed. 
Section 3.0 presents the SERI methodological approach to validation. All differences between measured and calculated results are attributed to four external and three internal error types in building energy analysis techniques. We define various levels of validation according to the degree of control exercised over these error sources. Additionally, we identify four types of extrapolations inherent in validation studies. The relative strengths and weaknesses of empirical, analytical, and comparative validation techniques are shown with respect to these extrapolations, the control of error sources, and the simulation process. Finally, we present a systematic approach to validation that includes these three validation techniques. When properly applied, the methodology assists in establishing the parametric range within which a building energy analysis simulation can be used with confidence.

Section 4.0 discusses the two comparative studies we conducted at SERI. In the first study, we compared the DOE-2.1, BLAST-2MRT, DEROB-3, and SUNCAT-2.4 programs by modeling a simple high- and low-mass direct gain building with Madison, Wisconsin, TMY (typical meteorological year) data. One of the programs yielded annual results significantly different from the others. We subsequently uncovered a flaw in the thermal solution algorithm that required the code author to extensively rewrite this program. The other three programs compared closely for annual results but differed somewhat in hourly temperature profiles.

In the second comparative study, DOE-2.1.BLAST-3.0, DEROB-4, and SUNCAT-2.4 were again compared except that Albuquerque, New Mexico, TMY data were also used. In this case we observed a large scatter in the annual results from all four programs. The large differences indicated the need for further investigation to determine which, if any, of these programs was correct.

Section 5.0 describes the analytical verification studies we conducted to determine if the differences between the building energy analysis simulations shown in the second comparative study could be attributed to the faulty numerical solution of specific heat transfer mechanisms.

A set of analytical solutions was derived for simple cases that could also be modeled by the computer programs. Any differences between the analytical solution and the results obtained with the simulation indicate a mistake in the numerical solution algorithm of the code for that specific combination of heat transfer mechanisms, given the boundary conditions.

The major test types were:

- Temperature decay

- Steady-state overall conductivity

- Infiltration

- Glazing assembly conductivity

- Glazing assembly transmissivity

- Mass charging by radiation. 
Except for DEROB-3, we found no major errors, which suggested that the differences uncovered in the comparative studies were due to differences in how the codes treat other aspects of the problem. Potential sources of variation include modeling of other mechanisms, component boundary conditions, component coupling, and other errors. Examples include infiltration, ground coupling, moisture, optics (external and internal), sky infrared, wind, internal convection, and external radiation (short and long wave). This also suggests the need for rigorous empirical validation with high-quality detailed data.

Section 6.0 describes the empirical data currently being collected that involve a $1000-\mathrm{ft}^{2}$ house moved to the SERI field test site from Denver, Colorado, and a $120-\mathrm{ft}^{2}$ two-zone test cell constructed at the test site. These facilities have been carefully instrumented with approximately 250 sensors in each. There is also a complete weather station at the site. Sensors consist of thermocouple rakes, differential thermocouple pairs, pyranometers, pyrheliometers, anemometers, heat-flux transducers, and shielded thermocouples. The data acquisition methodology was developed specifically to meet special validation data needs and in some cases involved the design of new measurement devices. Most notable of these was a multi-zone infiltration monitoring system. The data acquisition methodology has been adopted by all Class A validation data collection sites. We report on the empirical validation results in a forthcoming SERI publication, Empirical Validation: Using Measured Class A Data (SERI/TR-2S4-1840).

In Section 7.0 we compare four building energy analysis simulation programs (DOE-2.I, BLAST-3.0, DEROB-4, and SUNCAT-2.4) in several areas including user interface, solution technique, radiation modeling, convection and conduction modeling, systems, and scheduling. We also compared the programs on the basis of their relative adaptability to new algorithms for innovative design. This will be useful to those in the process of selecting a code for a particular application.

\section{Conclusions}

- Previous validation of computer simulations have not established the error to be associated with these simulations, nor have these studies indicated parameter ranges over which the error is not significant.

- Comparative studies show that large differences can exist between state-of-the-art simulations, even for a very simple passive solar building.

- Analytical studies prove that the component conduction mechanism is accurately treated (except for DEROB-3), indicating the differences between simulations are from other mechanisms that are not so easily modeled, such as radiation and internal convection.

- Empirical studies should measure key inputs, should propagate errors to define total problem uncertainty, and should resolve potential compensating errors with mechanism level data. 


\section{Table of Contents}

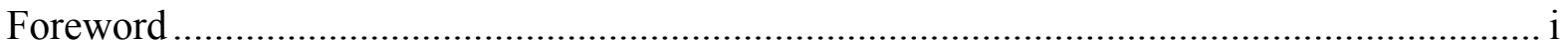

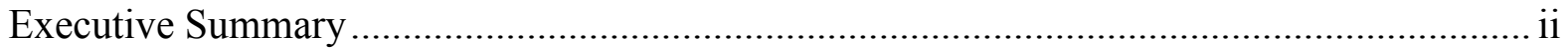

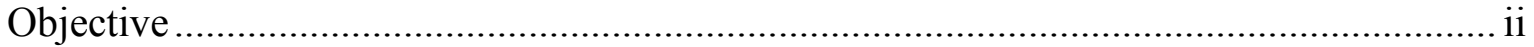

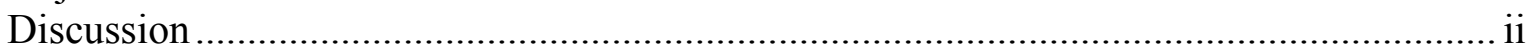

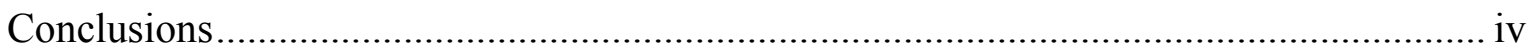

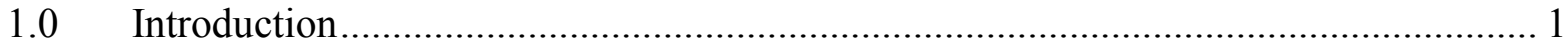

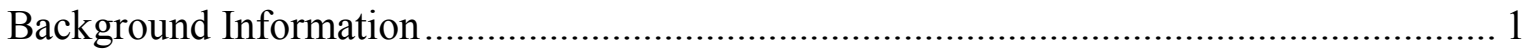

1.1 Historical Context of Building Energy Analysis Information ................................. 1

1.2 History of SERI Validation Work...................................................................... 4

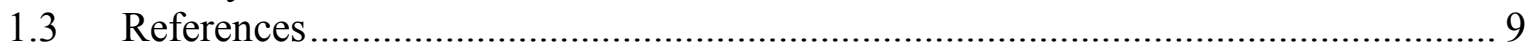

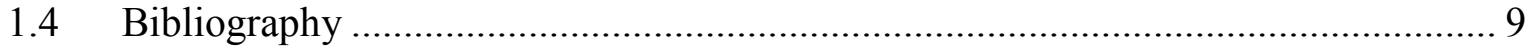

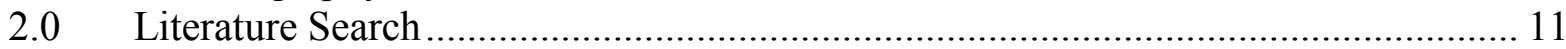

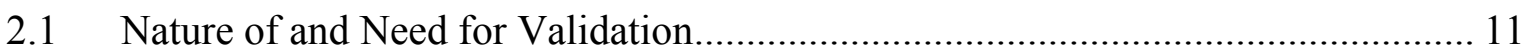

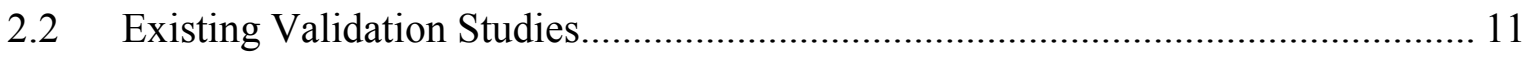

2.3 Suggestions for Further Validation ............................................................... 14

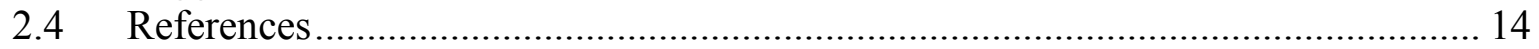

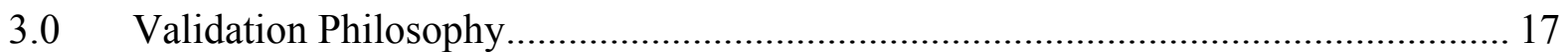

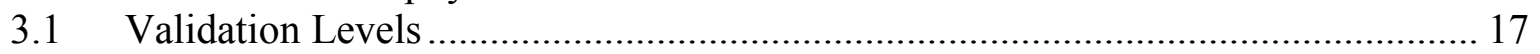

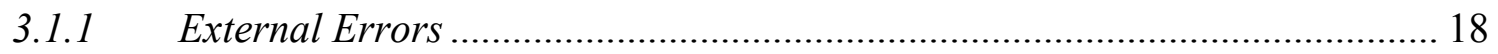

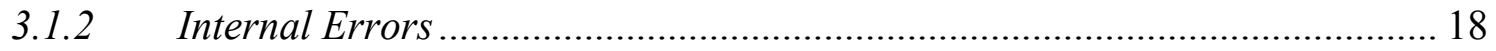

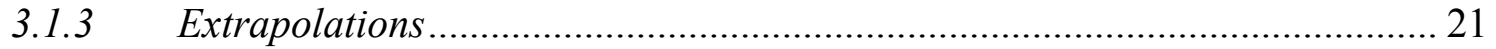

3.2 Methodological Approach ............................................................................. 23

3.2.1 Comparative Studies .............................................................................. 23

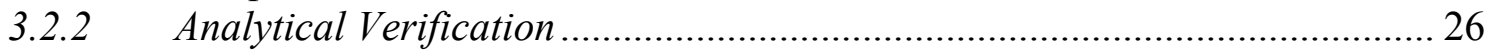

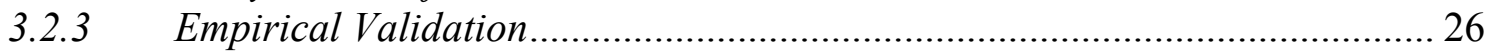

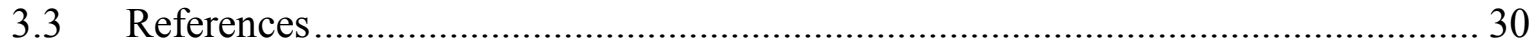

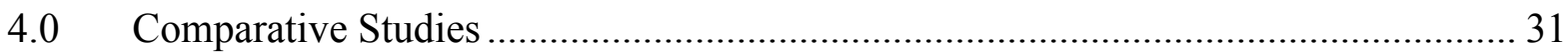

4.1 Phase I Comparative Study: DOE-2.1. BLAST-2MRT, DEROB-3, and

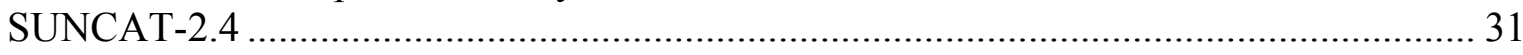

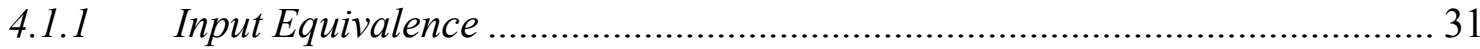

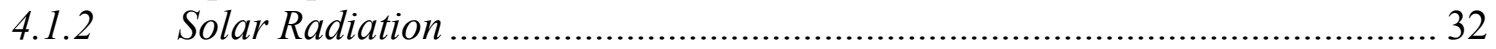

4.1.3 Hourly Free-Float Temperature Profiles …………………......................... 35

4.1.4 Annual Heating and Cooling Loads ........................................................... 36

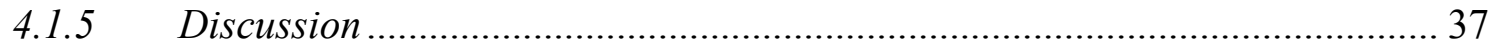

4.1.6 Conclusions ............................................................................................ 39

4.2 Phase II Comparative Study: DOE-2.1, BLAST-3.0, DEROB-4, and

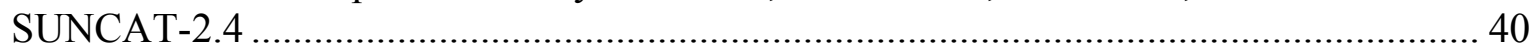

4.2.1 Test Building Characteristics ................................................................... 40

4.2.2 Annual Heating and Cooling Loads .......................................................... 41

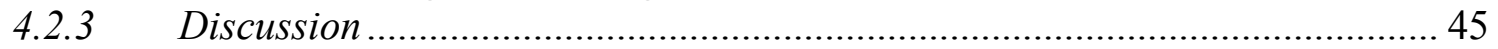

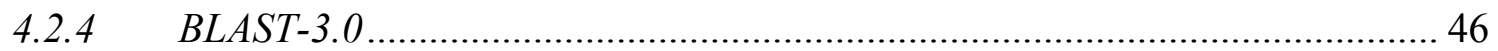

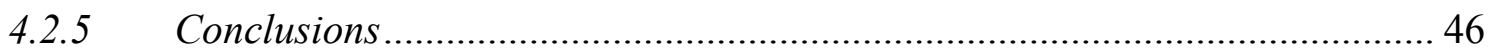

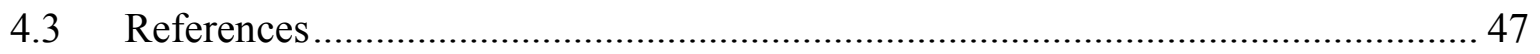

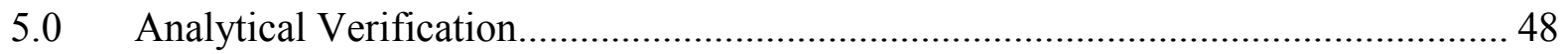




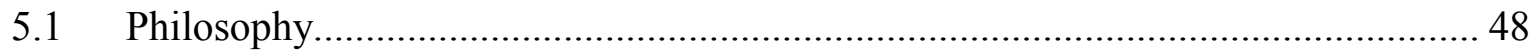

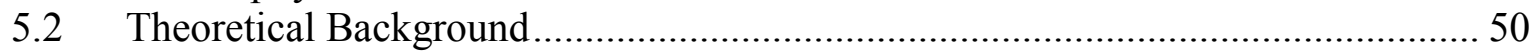

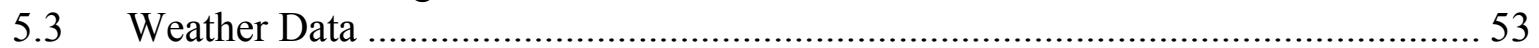

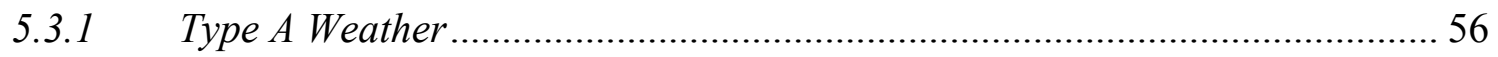

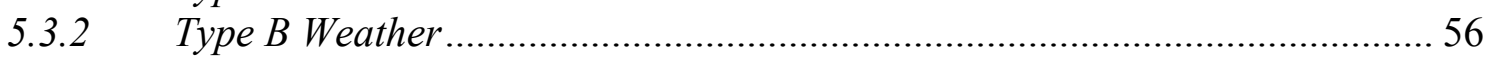

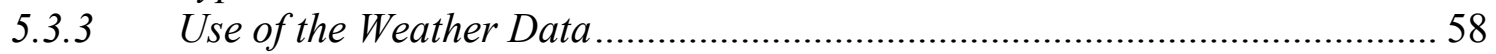

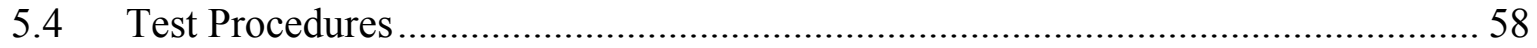

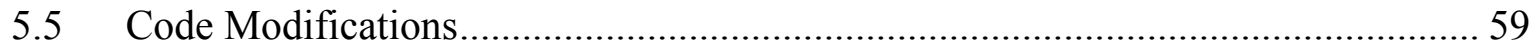

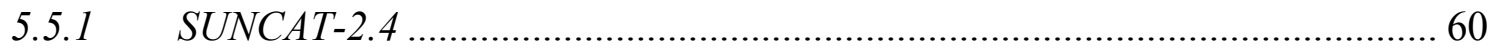

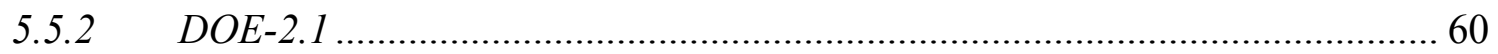

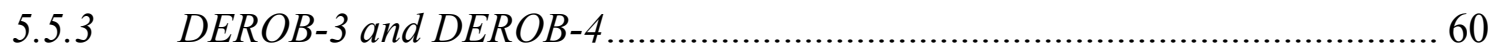

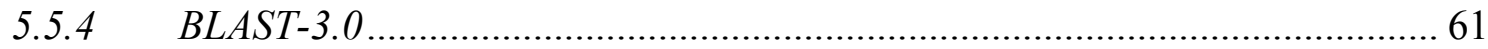

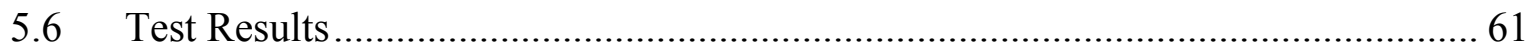

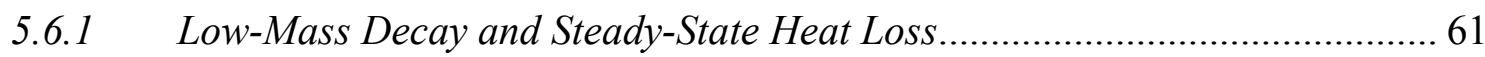

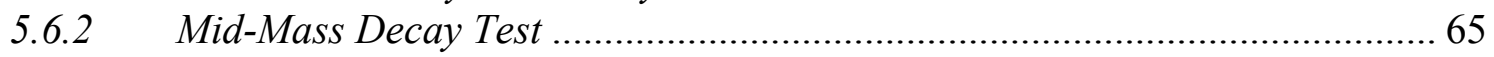

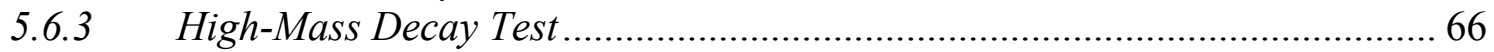

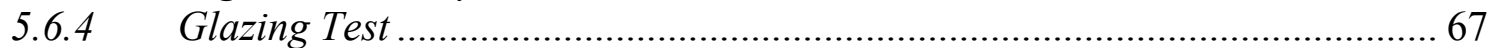

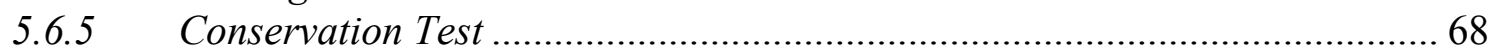

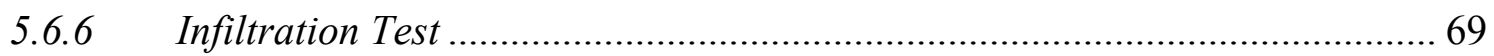

5.6.7 Solar Charging and Window Transmissivity ............................................. 71

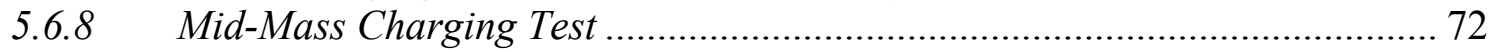

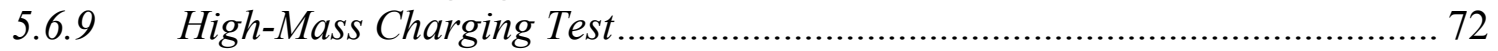

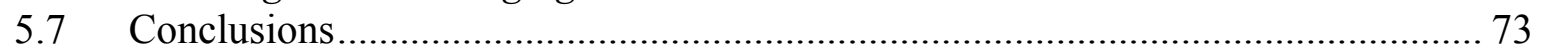

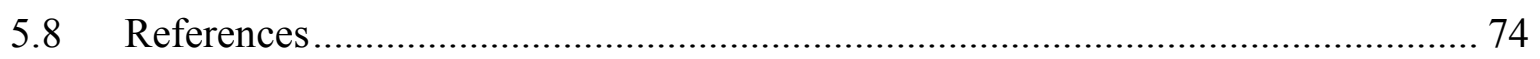

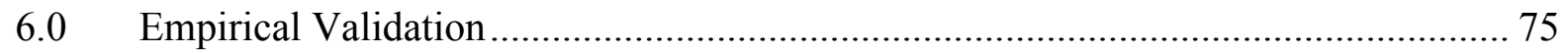

6.1 Empirical Validation Philosophy: Qualitative Presentation ..................................... 75

6.1.1 Take Measurements in Unoccupied Controlled Buildings ............................ 75

6.1.2 Minimize External Error Sources ................................................................ 78

6.1.3 Record Data for Individual Heat Transfer Mechanisms and the Overall

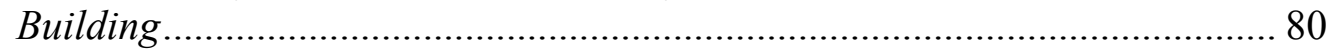

6.1.4 Modify Structure to Eliminate Inappropriate Mechanisms ............................ 81

6.1.5 Provide Consistency Checks on Data ....................................................... 82

6.1.6 Defined Methods for Comparison Including Effects of Measurement Errors 82

6.2 Implementation of Validation Philosophy: Technical Presentation ........................ 84

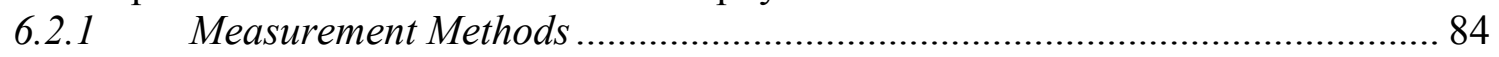

6.2.2 SERI Field Site Installation ..................................................................... 91

6.2.3 Minimizing External Error Sources: Measurement of Input Parameters ...... 98

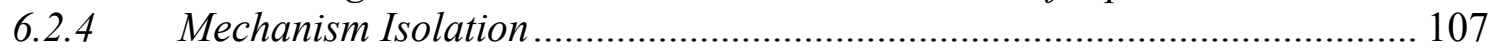

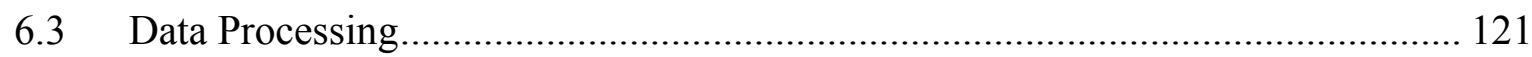

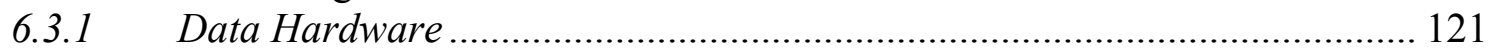

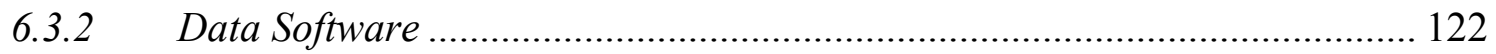

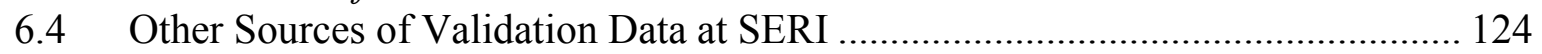

6.4.1 Performance Test Group Cells, Nos. 1 to 4 …….......................................... 124

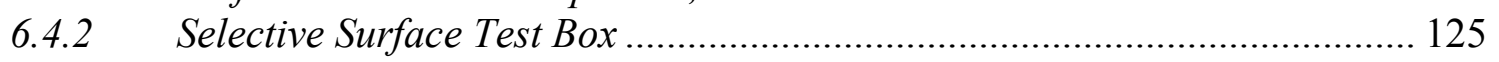

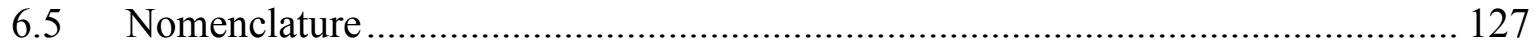

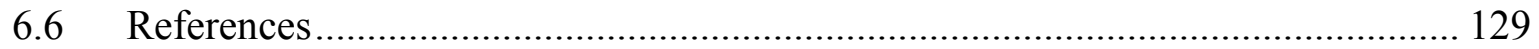




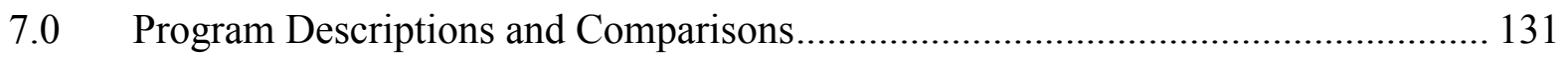

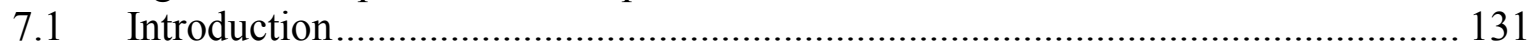

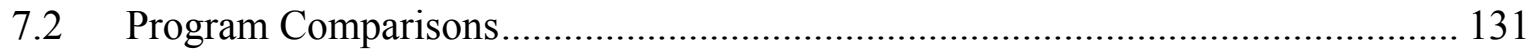

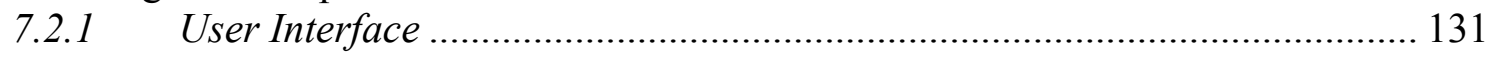

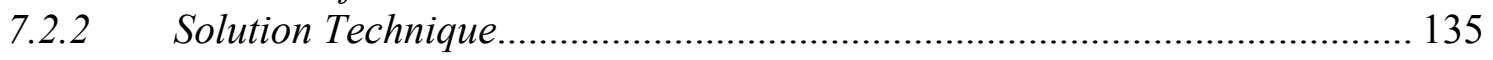

7.2.3 Radiation Modeling ……………………….......................................... 135

7.2.4 Convection and Conduction Modeling .................................................... 136

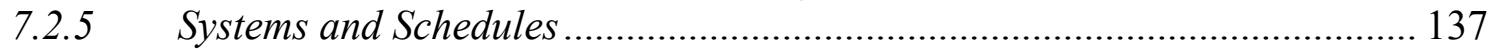

7.2.6 Passive Solar Applications ……………................................................... 137

7.3 SUNCAT 2.4

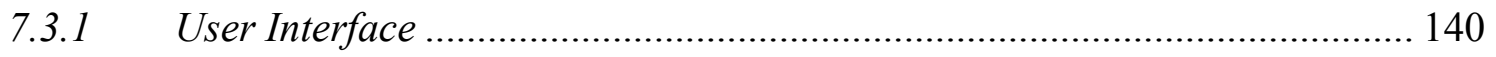

7.3.2 Solution Technique................................................................................. 141

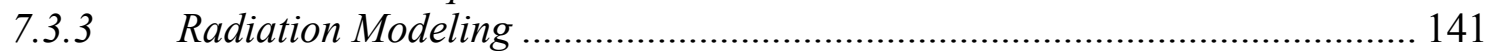

7.3.4 Convection and Conduction Modeling .................................................... 142

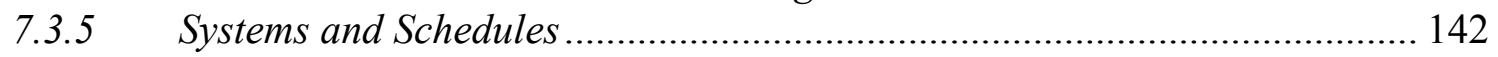

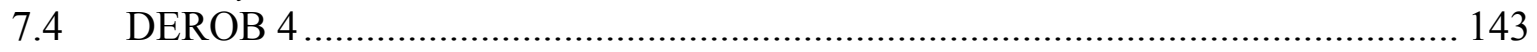

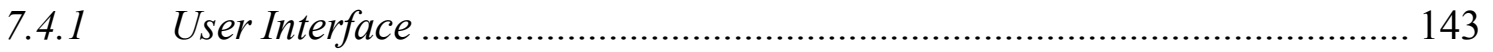

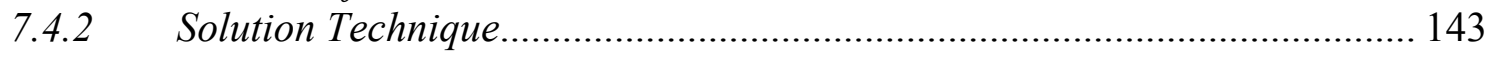

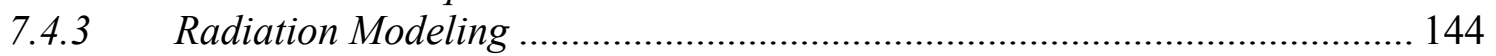

7.4.4 Convection and Conduction Modeling ………......................................... 145

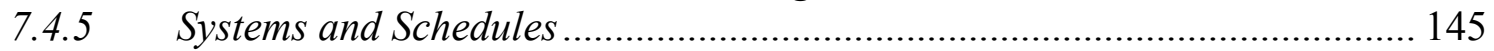

7.5 BLAST 3.0

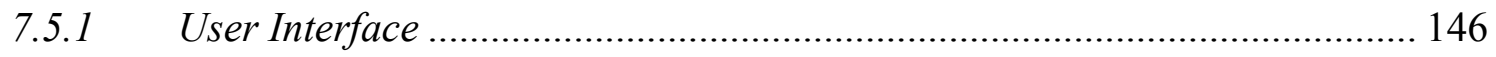

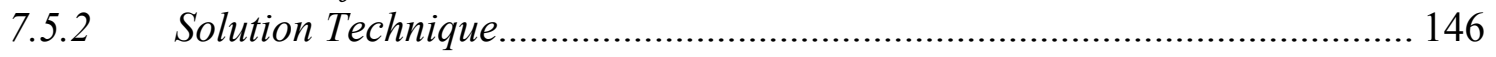

7.5.3 Radiation Modeling ………………………............................................. 147

7.5.4 Convection and Conduction Modeling ................................................... 147

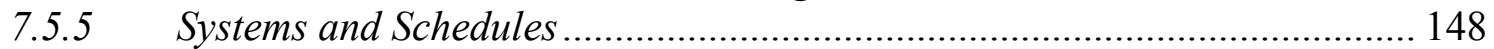

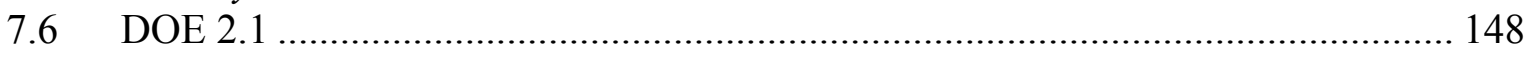

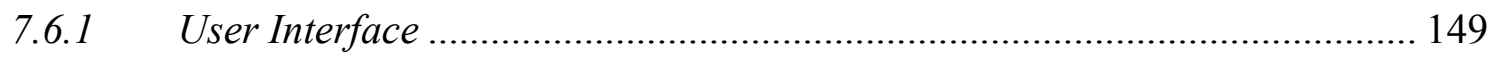

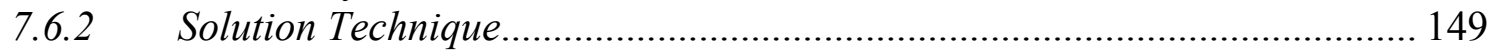

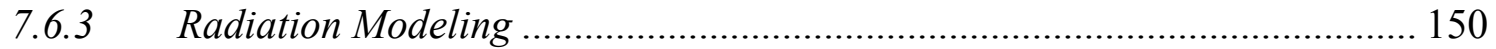

7.6.4 Convection and Conduction Modeling ..................................................... 151

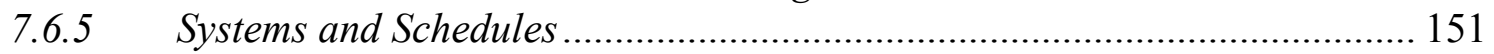

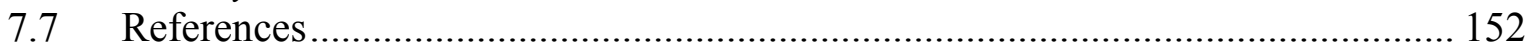

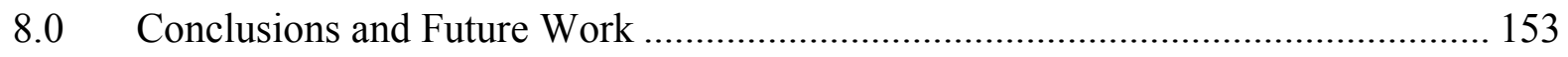

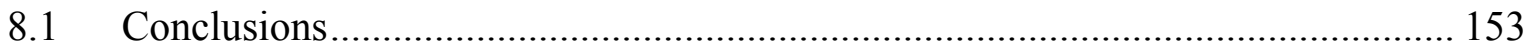

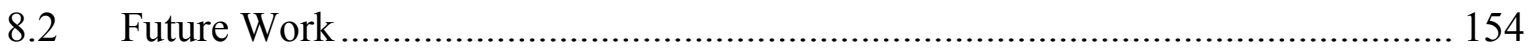

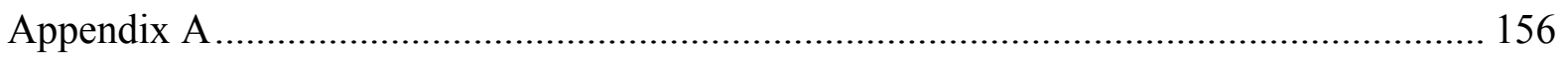

A.1 Low-Mass Decay and Steady-State Heat Loss Test ............................................. 156

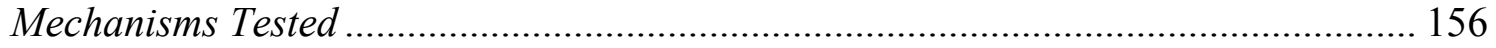

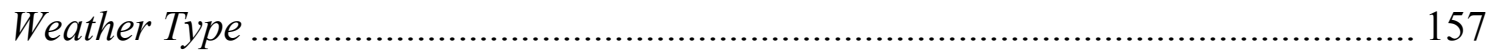

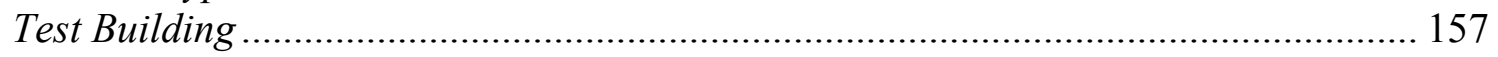

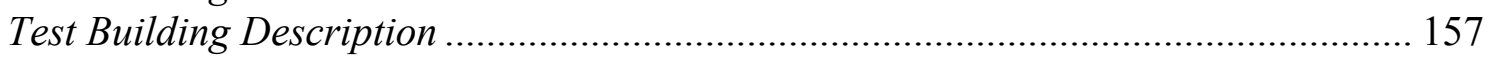

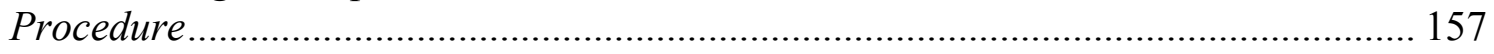

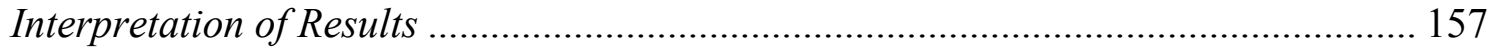

Notes on Implementation of the Test ......................................................................... 159 
A.2 Mid-Mass Decay and Steady-State Heat Loss Test........................................ 160

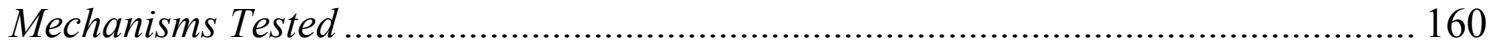

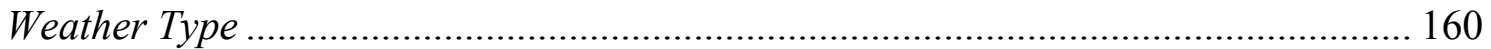

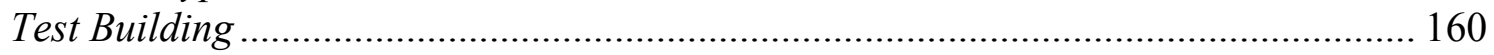

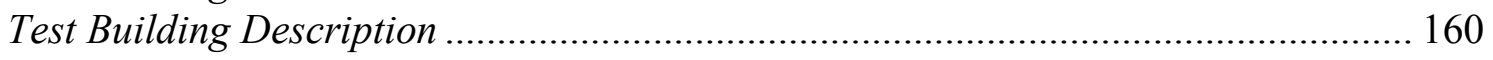

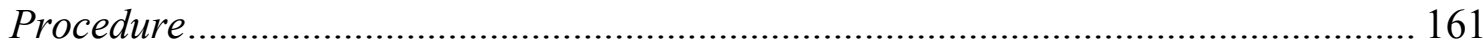

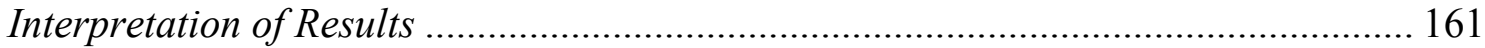

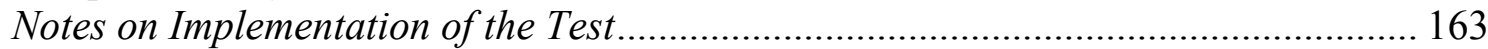

A.3 High-Mass Decay and Steady-State Beat Loss Test......................................... 163

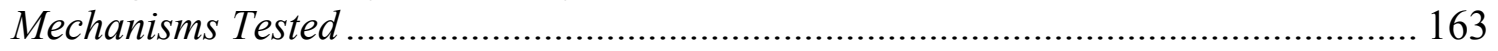

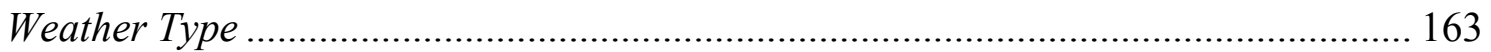

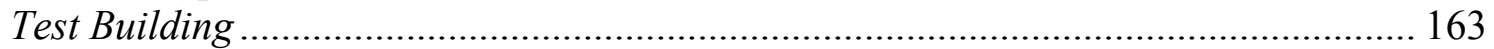

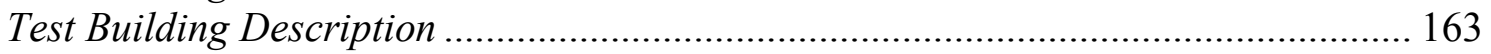

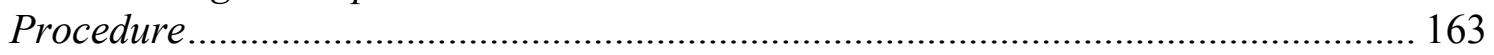

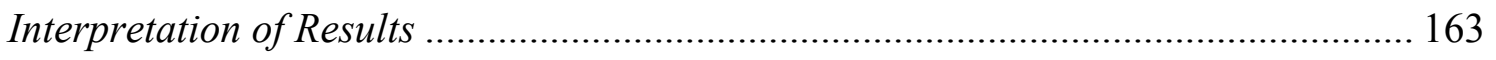

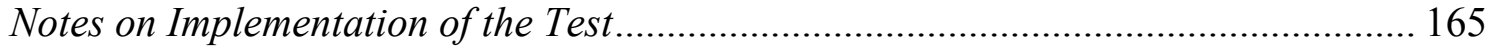

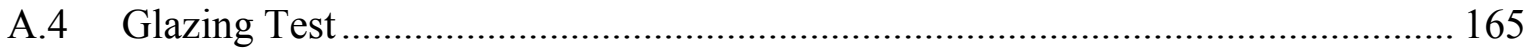

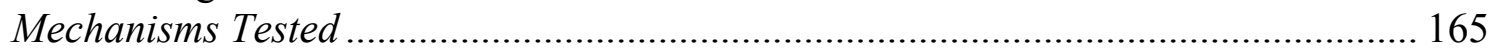

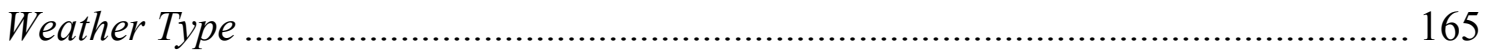

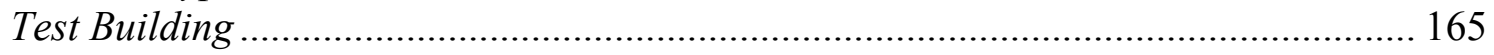

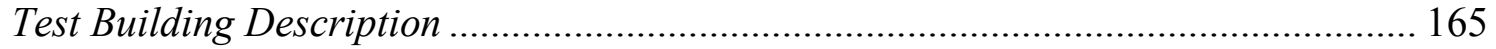

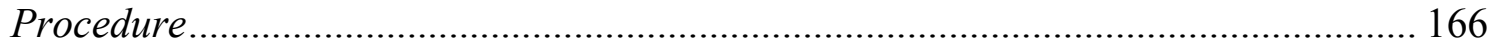

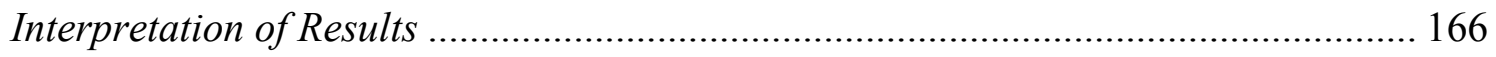

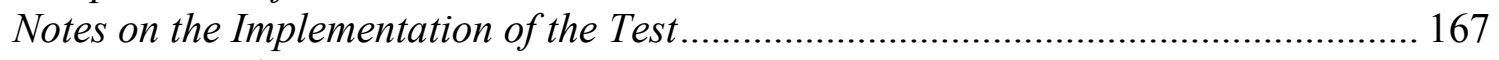

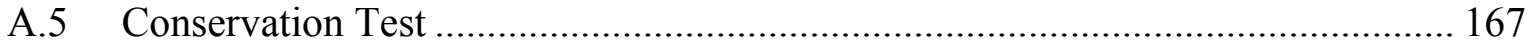

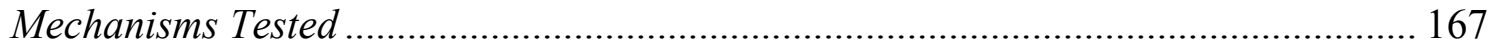

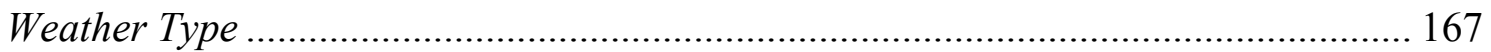

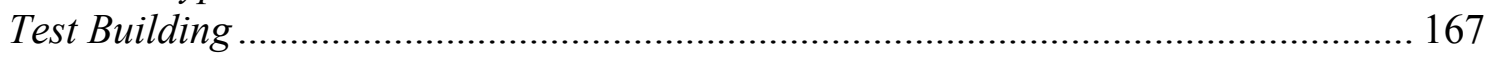

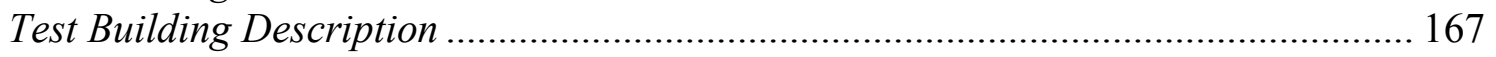

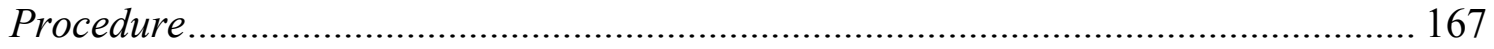

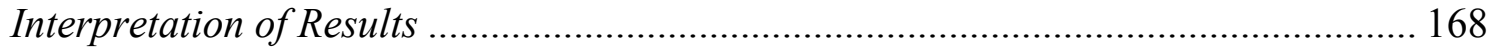

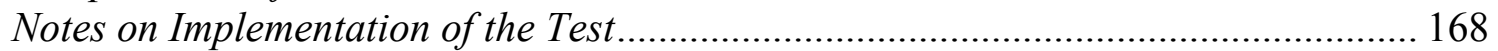

A.6 Infiltration Test ........................................................................................ 169

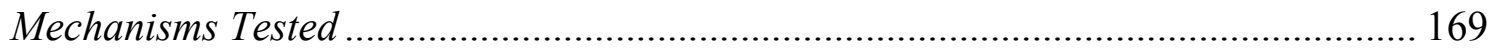

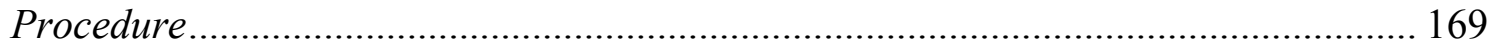

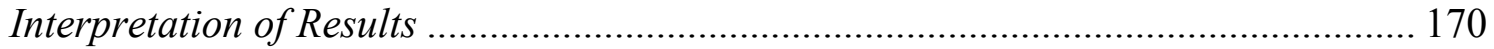

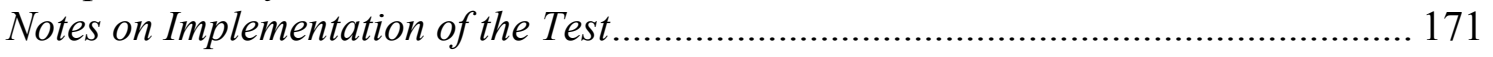

A.7 Solar, Charging, and Window Transmissivity Test ....................................... 171

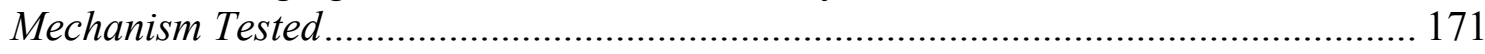

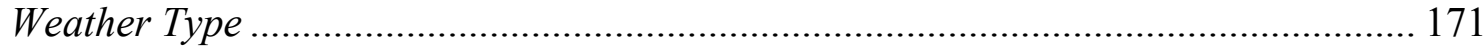

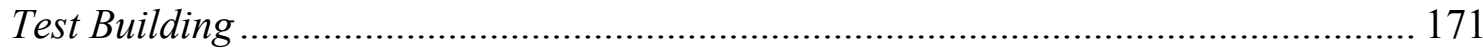

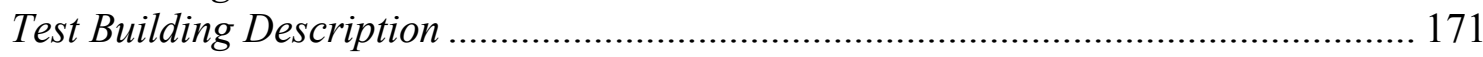

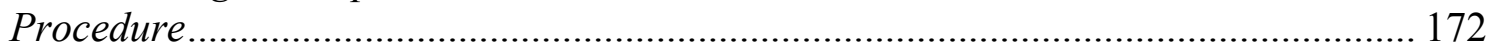

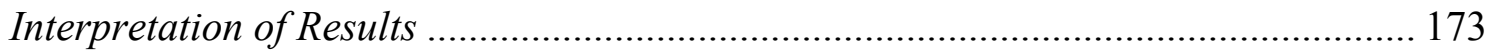

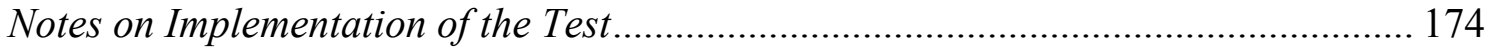




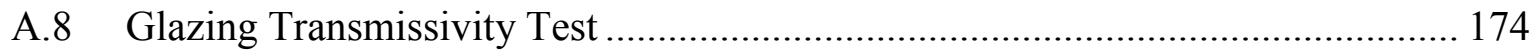

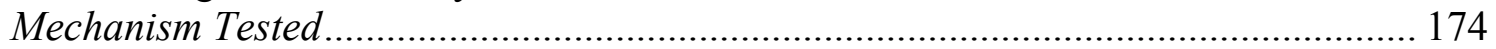

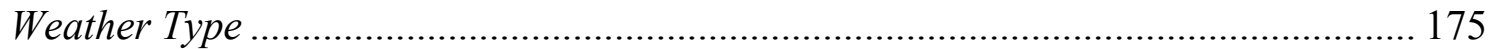

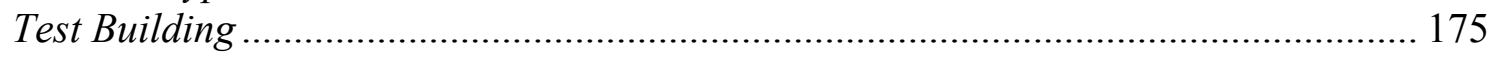

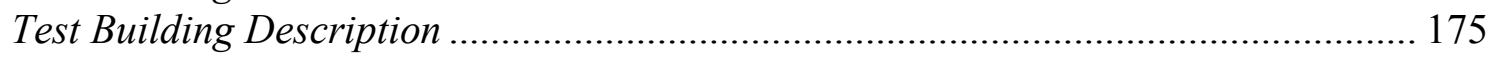

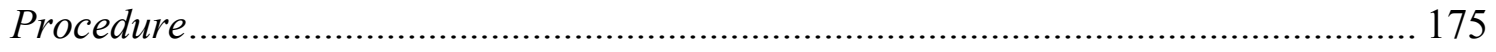

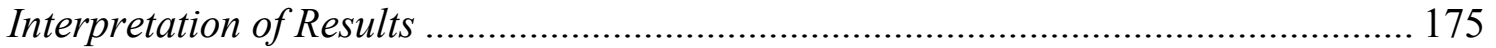

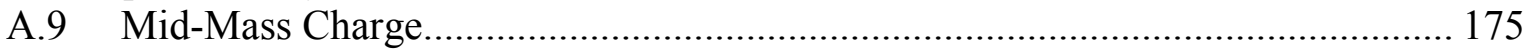

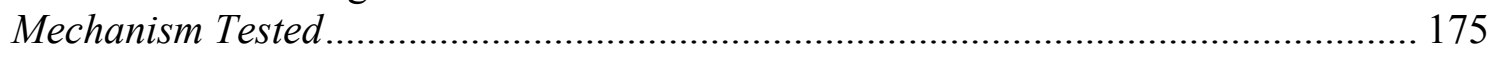

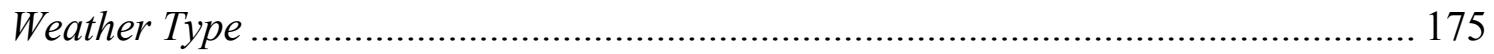

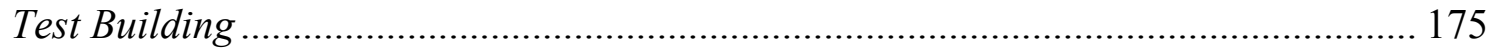

Test Building Description .............................................................................. 175

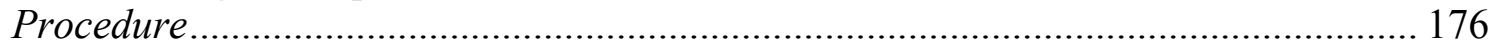

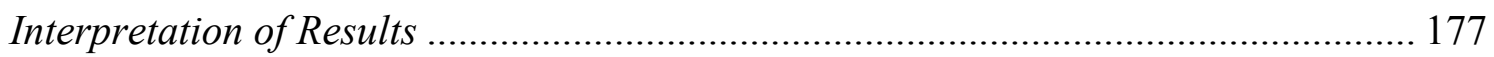

Notes on Implementation of the Test ........................................................................ 177

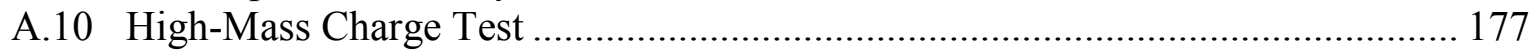

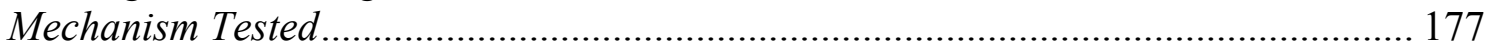

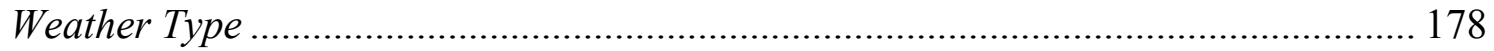

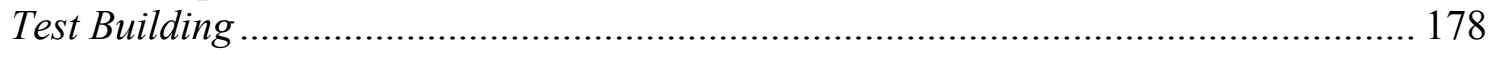

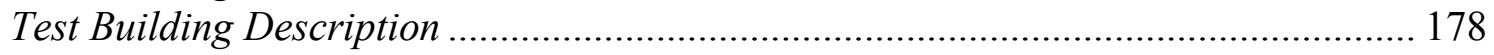

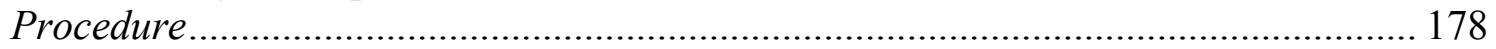

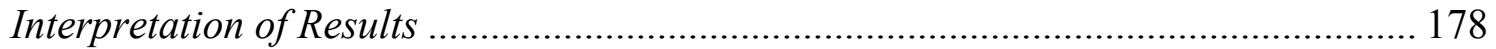

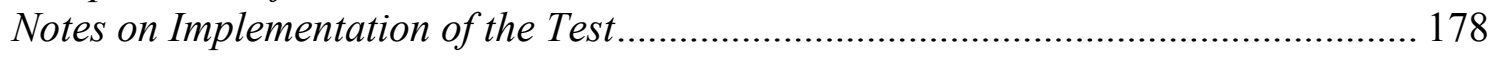

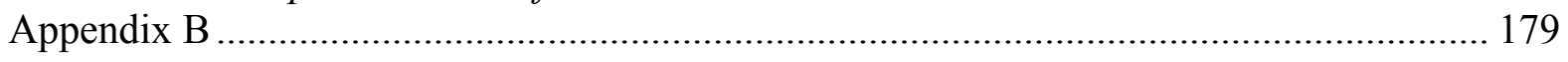




\subsection{Introduction}

\section{Background Information}

This section outlines the history of building energy analysis simulation as it pertains to the work done at the Solar Energy Research Institute (SERI). The section titled, History of SERI Validation Work summarizes the factors we considered when choosing which simulations to validate.

\subsection{Historical Context of Building Energy Analysis Information}

Using computer programs for building energy analysis is not new. Since the late 1960s, the number of computer programs in both the public and private sectors has proliferated. Figures 1-1 and 1-2 show this development. With almost 15 years of development behind some of these programs, it is tempting to conclude that they are sufficiently accurate. This is not true for several reasons.

Until the oil embargo of 1973, these programs were used for sizing heating, ventilating, and air conditioning (HVAC) equipment or for justifying to the consumer a new piece of equipment. Little emphasis was placed on the ability to predict envelope loads accurately in other than conventional building types. Therefore, the authors of these programs made simplifying assumptions and chose solution approaches that, although quite reasonable for computational efficiency, were not adequate for innovative building designs.

In 1973, it became evident that the approach to the use of energy in buildings would have to change. At first, the trend was toward active solar systems that presented little difficulty for building energy analysis simulations (BEAS), since the solar components could be added much as another HVAC system. The original TRNSYS program was exclusively an active solar system simulation and was incorporated into such BEAS as DOE [1,2] and BLAST $[3,4]$ without necessitating major revamping of these programs.

By 1976-1977, more attention was being devoted to passive and innovative design strategies involving architectural modification of the building design to reduce load and to use environmental sources and sinks of energy. However, the existing BEAS were no longer appropriate under these new conditions. Adding new subroutines to existing programs did not ensure accurate energy analysis. Instead, fundamental changes to the analysis approach were often necessary to handle these innovative design strategies. For example, a major overhaul of the DOE code was required to accurately analyze strongly solar-driven, thermally massive buildings. The DOE program still does not include Trombe walls, even though they are a popular passive solar design. 


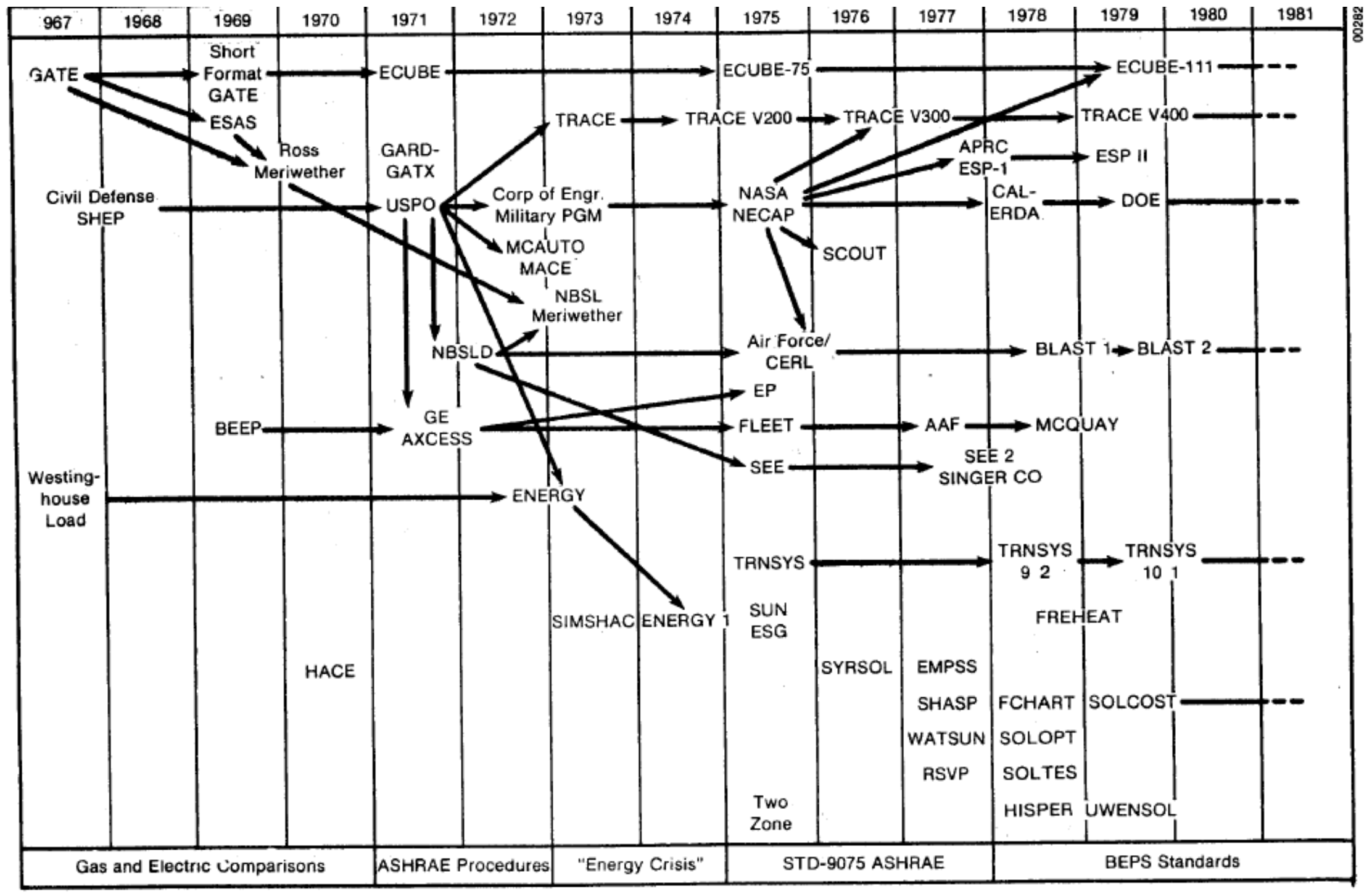

Figure 1-1. History of energy analysis 


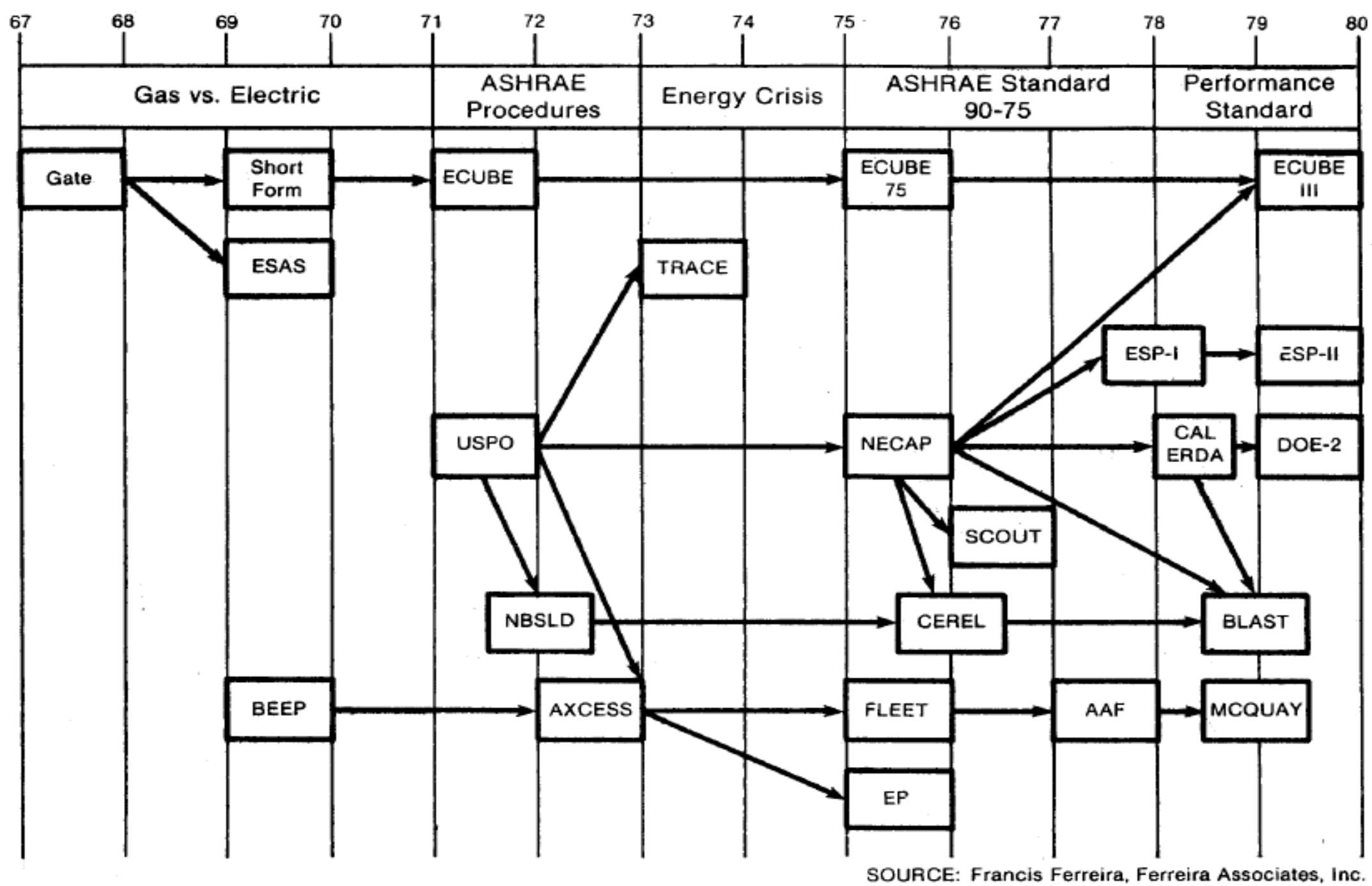

Figure 1-2. History of energy analysis computer programs 
The attempt on the part of code developers to accurately analyze passive design options has fostered an entirely new generation of building energy analysis simulations. There is still considerable controversy about what techniques are optimal. Calculating the impact of a wide variety of innovations on total building load will involve considerable study of building physics and the development of new algorithms. The question of validation is crucial because there has not yet been time for extensive testing and application of this new generation of BEAS. Nevertheless, many simplified design tools, energy audit procedures, and rules of thumb are already being generated from these simulations. These tools will have a significant impact on the design of new buildings and the retrofit of existing buildings. Inaccuracies in these tools could have a negative impact on public acceptance of energy-efficient systems.

\subsection{History of SERI Validation Work}

This task was not originally intended to validate building energy simulations. In 1978, we surveyed the state-of-the-art of passive solar design and analysis tools and selected several to be installed at SERI for use in parametric studies, design tool development, and design evaluation. At that time we shared the view expressed by the U.S. Department of Energy (DOE) and other researchers that the building energy simulations were generally reliable with small modifications needed to increase analysis capability for passive buildings.

We began our survey with a review of manual and programmable calculator techniques. The methods surveyed are shown in Tables $1 \mathrm{~A}$ and $1 \mathrm{~B}$.

The survey consisted of a telephone call to the author of the program in which the following questions were asked:

- Describe the hardware necessary to run the method. Can it be done manually or with a four-function calculator? If not, specify the required equipment as follows:

○ Programmable calculator, brand, and model number

- Microcomputer, brand, model number, language, core

○ Minicomputer, brand, model number, language, core

- Mainframe computer, brand, model number, language, core.

- Describe the solution technique.

- Describe the target audience and level of expertise required to understand the documentation and run the method.

- Describe the documentation.

- Describe the capabilities of the method for analysis of passive energy strategies. What building types and systems are appropriate? Can multi-zone buildings be analyzed?

- Describe limitations and built-in assumptions of the method.

- Describe near-term planned improvements.

- Describe user support services.

- Describe other methods you know of. 
Table 1A. Survey of Passive Design Tools for Manual and Programmable Calculators (Descriptions, Authors, and Availability)

\begin{tabular}{|l|l|c|c|l|}
\hline \multicolumn{1}{|c|}{ Description } & \multicolumn{1}{c|}{ Authors } & \multicolumn{2}{c|}{ Availability } \\
\cline { 3 - 5 } & \multicolumn{1}{|c|}{$\begin{array}{c}\text { Cost } \\
\text { (\$) }\end{array}$} & Date & \\
\hline TEANET & $\begin{array}{l}\text { J.T. Kohler and } \\
\text { P.W. Sullivan }\end{array}$ & 95 & 1978 & $\begin{array}{l}\text { Total Environmental Action, Inc. } \\
\text { Church Hill } \\
\text { Harrisville, NH 04350 }\end{array}$ \\
\hline PEGFIX and PEGFLOAT & W. Glennis & 75 both & $1978 \begin{array}{l}\text { Princeton Energy Group } \\
729 \text { Alexander Road } \\
\text { Princeton, NJ 08540 }\end{array}$ \\
\hline Solarcon Program ST33 & R.W. Graeff & 138 & 1979 & Solarcon, Inc. \\
\hline $\begin{array}{l}\text { A Design Handbook for Direct Heat Transfer Passive } \\
\text { Solar Systems }\end{array}$ & R.M. Lebens & 10 & 1978 & $\begin{array}{l}\text { Northeast Solar Energy Association } \\
\text { P.O. Box 541, 22 High Street } \\
\text { Brattleboro, VT 05301 }\end{array}$ \\
\hline $\begin{array}{l}\text { A Simple Empirical Method for Estimating the } \\
\text { Performance of a Passive Solar Heated Building of the } \\
\text { Thermal Storage Wall Type }\end{array}$ & $\begin{array}{l}\text { J.D. Balcomb and } \\
\text { R.D. Farland }\end{array}$ & -- & 1978 & $\begin{array}{l}\text { Report \#LA-UR-78-1159 } \\
\text { Available from NTIS } \\
5285 \text { Port Royal Road } \\
\text { Springfield, VA 22161 }\end{array}$ \\
\hline SEEC VI - Passive Solar Heating & $\begin{array}{l}\text { C.B. Winn, D. Barley, } \\
\text { G. Johnson, J. Lellar }\end{array}$ & 125 & 1978 & Solar Environmental Engineering Co., Inc. \\
\hline $\begin{array}{l}\text { Sunshine Power Programs for Modeling Solar Energy } \\
\text { Components and Systems }\end{array}$ & G. Shrameck & $30-60$ & 1977 & $\begin{array}{l}\text { Sunshine Power Co. } \\
1018 \text { Lancor Drive } \\
\text { San Jose, CA 95129 }\end{array}$ \\
\hline $\begin{array}{l}\text { Mazria Design Patterns (Rule-of-Thumb) in The } \\
\text { Passive Solar Energy Book }\end{array}$ & E. Mazria & 11 & 1979 & $\begin{array}{l}\text { Rodale Press } \\
\text { Emmaus, PA 18049 }\end{array}$ \\
\hline $\begin{array}{l}\text { An Averaging Technique for Predicting the } \\
\text { Performance of a Solar Energy Collector System }\end{array}$ & G.H. Stickford & -- & 1976 & Sharing the Sun, Vol. 4, 1976, pp. 295-315 \\
\hline
\end{tabular}


Table 1B. Survey of Passive Design Tools for Manual and Programmable Calculators (Applications, Column Types, System Types, Tools Required, Bases of Method, and Output)

\begin{tabular}{|c|c|c|c|c|c|c|c|c|c|c|c|c|c|c|c|c|c|c|}
\hline \multirow[t]{2}{*}{ Description } & \multicolumn{4}{|c|}{ Application } & \multicolumn{2}{|c|}{$\begin{array}{l}\text { Column } \\
\text { Type }\end{array}$} & \multicolumn{2}{|c|}{$\begin{array}{l}\text { System } \\
\text { Type }\end{array}$} & \multicolumn{4}{|c|}{ Tools Required } & \multicolumn{3}{|c|}{$\begin{array}{l}\text { Basis of } \\
\text { Method }\end{array}$} & \multicolumn{3}{|c|}{ Output } \\
\hline & 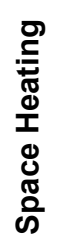 & 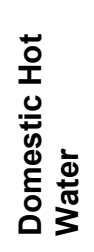 & 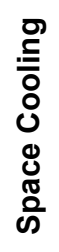 & $\frac{0}{\frac{0}{E}} \frac{n}{n}$ & $\frac{\overline{0}}{\frac{0}{\partial}}$ & 言 & $\sum_{\substack{0 \\
\hdashline}}^{0}$ & 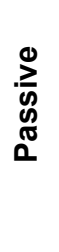 & $\begin{array}{l}\frac{0}{0} \\
\frac{0}{0} \\
\frac{0}{0} \\
\frac{0}{0} \\
\frac{c}{0} \\
\frac{0}{\pi} \\
\frac{0}{0}\end{array}$ & 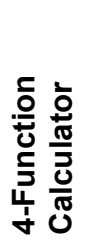 & 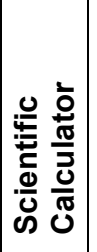 & $\begin{array}{l}\frac{0}{0} \\
\frac{0}{\pi} \\
\frac{\varepsilon}{\varepsilon} \\
\frac{0}{\pi} \\
\frac{\pi}{5} \\
\frac{0}{2} \\
\frac{0}{\pi} \\
0 \\
0\end{array}$ & ס & 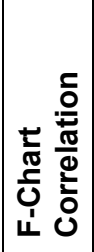 & 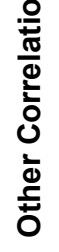 & 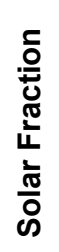 & 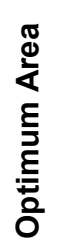 & 衣 \\
\hline TEANET & $\bullet$ & & & & & & & $\bullet$ & & & & $\bullet$ & $\bullet$ & & & & & $\bullet$ \\
\hline PEGFIX and PEGFLOAT & $\bullet$ & & & & & & & $\bullet$ & & & & $\bullet$ & $\bullet$ & & & & & $\bullet$ \\
\hline Solarcon Program ST33 & $\bullet$ & & & & & & & $\bullet$ & & & & $\bullet$ & $\bullet$ & & & $\bullet$ & & \\
\hline $\begin{array}{l}\text { A Design Handbook for Direct } \\
\text { Heat Transfer Passive Solar } \\
\text { Systems }\end{array}$ & $\bullet$ & & & $\bullet$ & & & & $\bullet$ & $\bullet$ & & & $\bullet$ & $\bullet$ & & & $\bullet$ & & \\
\hline $\begin{array}{l}\text { A Simple Empirical Method for } \\
\text { Estimating the Performance of } \\
\text { a Passive Solar Heated } \\
\text { Building of the Thermal } \\
\text { Storage Wall Type }\end{array}$ & $\bullet$ & & & & & & & $\bullet$ & $\bullet$ & $\bullet$ & & & & & $\bullet$ & $\bullet$ & & \\
\hline $\begin{array}{l}\text { SEEC VI - Passive Solar } \\
\text { Heating }\end{array}$ & - & & & $\bullet$ & & & & $\bullet$ & & & & $\bullet$ & & & $\bullet$ & $\bullet$ & & \\
\hline $\begin{array}{l}\text { Sunshine Power Programs for } \\
\text { Modeling Solar Energy } \\
\text { Components and Systems }\end{array}$ & $\bullet$ & $\bullet$ & & $\bullet$ & $\bullet$ & $\bullet$ & $\bullet$ & $\bullet$ & & & & $\bullet$ & $\bullet$ & $\bullet$ & $\bullet$ & $\bullet$ & & $\bullet$ \\
\hline $\begin{array}{l}\text { Mazria Design Patterns (Rule- } \\
\text { of-Thumb) in the Passive Solar } \\
\text { Energy Book }\end{array}$ & $\bullet$ & & & & $\bullet$ & $\bullet$ & & $\bullet$ & $\bullet$ & & & & $\bullet$ & & & $\bullet$ & $\bullet$ & $\bullet$ \\
\hline $\begin{array}{l}\text { An Averaging Technique for } \\
\text { Predicting the Performance of } \\
\text { a Solar Energy Collector } \\
\text { System }\end{array}$ & $\bullet$ & $\bullet$ & & & $\bullet$ & $\bullet$ & . & $\bullet$ & $\bullet$ & & $\bullet$ & & $\bullet$ & & & $\bullet$ & & \\
\hline
\end{tabular}


We followed this phone survey with a written order for documentation and software (in the case of automated techniques). Installation of the method was considered complete when after a careful review of the documentation we were able to achieve a benchmark run that agreed with the sample problem provided by the author. Any discrepancies, bugs, or anomalies discovered in the documentation or method were discussed with the authors until resolved.

As a result of this survey we drew several conclusions:

1. Advertisements in trade journals and discussions with authors gave the impression that a method was more powerful and less limited than we found in practice.

2. The number of bugs, anomalies, and inconsistencies found in the documentation and software indicated that these techniques were more developmental than expected.

3. None of the manual or programmable calculator methods were comprehensive or flexible enough for our research purposes. We needed a more detailed level of simulation.

As a result of conclusion 3, we began to survey the state-of-the-art of detailed hourly building energy simulations, focusing on those computer programs that claimed some degree of passive analysis capability. We used the same surveying methodology as with the simpler techniques except that we used both the initial telephone contact and the review of documentation to reduce the number of codes we would have to install on the SERI computer. The cost of installing these large programs (e.g., DOE-2.1 contains more than 60,000 card images) restricted us to a maximum of four codes. Because the documentation review was such an important part of the selection process for the large codes, we attempted to formalize the process by borrowing appropriate techniques from previous reviews and surveys [5-10].

We chose the following criteria to guide our selection of programs for installation on the SERI computer:

- Public domain (or university)

- Overall building energy analysis capabilities

- Passive analysis capabilities

- User characteristics

- Documentation

- Variety of modeling and solution techniques.

We decided to restrict our inquiry to public domain or university programs to avoid legal difficulties, reduce costs, and ensure that other researchers would have access to the algorithms in these codes. The evaluation of overall building energy analysis capabilities focused on envelope load and included an assessment of how easy it would be to add additional passive analysis capabilities as algorithms became available. User characteristics included ease and speed of input, output report capabilities, and clarity of documentation.

Finally, we chose programs to represent different modeling and solution approaches as a quality control measure. For example, we gain little if two programs that use similar assumptions and 
solution techniques agree; however, if programs using different assumptions and solution approaches agree, confidence in our understanding of the physical systems increases.

Based on these criteria, we narrowed the field to the following programs:

- PASOLE, developed by Los Alamos National Laboratory (LANL), Q-11 Group

- DEROB, developed by Francisco Arumi Noe, University of Texas at Austin

- BLAST, developed by the Corps of Engineers Construction Engineering Research Laboratory and Lawrence Berkeley Laboratory (CERL/LBNL)

- DOE, developed by the DOE Los Alamos National Lab WX-4 Group, and Lawrence Berkeley National Laboratory DOE/LANL WX-4/LBL

- SUNCAT, developed by NCAT, Larry Palmitre/Terry Wheeling, National Center for Appropriate Technology

- UWLITE, developed by Ashley Emery and Deen Heerwaagen, University of Washington

- UWENSOL, developed by Ashley Emery and Deen Heerwaagen, University of Washington

- SOLPAS, developed by Martin Marietta Corporation.

We eliminated SOLPAS because it was too restrictive in the type of building for which it could be used and PASOLE because it was weak in user interface and documentation. SUNCAT appeared to have equal capabilities with much better user characteristics than PASOLE. We eliminated UWLITE and UWENSOL when we learned that neither the software nor the documentation would be developed enough for our use during this project. DOE, BLAST, DEROB, and SUNCAT met our criteria.

The DOE program represented a response factor technique. BLAST-3.0 represented an approach in which the thermal response of the walls was found using response factors, and zone interactions were calculated with a nodal network approach. DEROB and SUNCAT both used a finite differencing approach. DEROB used implicit integration for wall nodes and a set of simultaneous equations for zone effects. SUNCAT used explicit integration for wall nodes and energy balance equations for air temperature. The energy balance equations were solved simultaneously for a maximum of two zones coupled advectively (see Section 7.0 for details). These four programs were representative of the various modeling and solution approaches common to building energy analysis simulations.

Once we had completed the installation and benchmarking of these four programs, we began a small comparative study (see Section 4.0 for details) to gain experience with these complex simulations. We chose a simple direct gain building with a high- and low-mass parametric option and entered an equivalent building description as input to each of the four computer programs. We expected similar results from these yearly simulations for all four codes; any differences would indicate input errors caused by our inexperience with these codes. Instead, we found that even for a very simple building disagreement in the calculation of annual loads of $\pm 25 \%$ was common. In some cases the spread was as high as $60 \%$. These unexpected results emphasized the 
need for detailed validation work beyond the limited studies already done by independent code authors. We decided to deemphasize the applied use of BEAS and instead focus our efforts on the development of a validation methodology. The four BEAS-DOE, BLAST, SUNCAT, and DEROB - would be used to both test and be tested by the validation methodology as it was developed.

\subsection{References}

1. Klein et al. BLAST User Reference Manual. Champaign, IL: University of Illinois at Urbana.

2. York, D.A. and Capiello, C.C. 1981. DOE-2 Engineers Manual, Ver. 2.1A. Los Alamos, NM: Los Alamos National Laboratory.

3. Merriam, R.L. and Feldmand, S.J. 19??. A.D. Little Inc., Solar Heating and Cooling System Simulation Programs. Palo Alto, CA: Electric Power Research Institute.

4. Smith, Hinchman, and Grylls Associates Inc. 19?? Value Engineering Analysis of Computer Simulation Energy Profile Systems for Institutional and Administrative Buildings. NASA.

5. Verstegen, P.L. 1978. Survey of Currently Used Simulation Methods. McLean, VA: Science Applications, Inc.

6. Solar/Passive Building Design Computer Programs: A Brief Survey with Comments. 1979. New England Solar Energy Association.

7. Cuba, J.F. and Crall, G. 1975. Bibliography on Available Computer Programs in the General Area of Heating, Refrigerating, Air Conditioning, and Ventilating, ASHRAE Research Project GRP-153. Columbus, OH: Battelle Laboratories, Rann Document Center National Science Foundation.

8. Science Applications, Inc. 1978. Survey of Component Modeling Activities. McLean, VA: SAI.

\subsection{Bibliography}

Boston Architectural Center. 1964. Architecture and the Computer: Proceedings BAC Conference, December 5, 1964. Boston, MA: Boston Architectural Center.

Forwood, B. 1971. The Development of a Computer-Aided Building Environment and Services Simulation Model, Sydney, Australia: Department of Architectural Science, University of Sydney.

Fulbright, B.E., Ferden, S.L., and Stalling, R.D. 1975. ESOP (Energy Systems Optimization Program): Its Description and Comparative Analysis, Houston, TX: NASA Johnson Space Center.

Government Services Administration. 1977. Energy Conservation Computer Software. Washington, DC: GSA.

Graven, R.M. 1974. A Comparison of Computer Programs Used for Modeling Solar Heating and Air-Conditioning Systems for Buildings. Berkeley, CA: Lawrence Berkeley Laboratory, University of California. 
Kusuda, T. 1971. Use of Computers for Environmental Engineering Related to Buildings. Washington, DC: National Bureau of Standards.

Lee, K., Teicholz, E., and Stewart, C. 1970. Computer Architecture Programs. Boston, MA: Center for Environmental Research.

Lokmanhekim, M. and Henninger, R.H. 1972. "Computerized Energy Requirement Analysis and Heating/Cooling Load Calculations of Buildings," ASHRAE Journal, April.

Lokmanhekim, M., ed. 1971. Procedure for Determining Heating and Cooling Loads for Computerized Energy Calculations--Algorithms for Building Heat Transfer Subroutines, ASHRAE.

McClure, C.J.R. 1971. “Optimizing Building Energy Use,” ASHRAE Journal, September.

National Electrical Contractors Association, Inc., Basics of Building Automation. 1973. Washington, DC: National Electrical Contractors Association, Inc.

National Science Foundation: Solar Heating and Cooling for Buildings Workshop: Part I; Technical Sessions, Washington, DC, 21-22 March 1973.

Negroponte, N. 1971. The Architecture Machines: Toward a More Humane Environment. Cambridge, MA: MIT Press.

North Carolina Research Group. 1968. AIA Computer Research Survey 1967-1968.

Oak Ridge National Laboratory/NSF. 1974. Inventory of Current Energy Research and Development. Vol. I, II, and III. U.S. GPO. Stock Numbers 5270-02174 and 4270-02175.

Reinschmidt, K.F. 1974. "Computer Methods for Building System Design," Presented at the ASCE National Meeting on Structural Engineering, Baltimore, Maryland, 19-23 April, 1974.

Science Applications, Inc. 1979. Validation Status of Solar Heating and Cooling Systems Models. McLean, VA: SAI, March.

Webster et al., 1978. User Perspectives of Selected Solar Design Tools. Los Alamos, NM: Los Alamos Scientific Laboratory 


\subsection{Literature Search}

This section describes some of the recent work done in the area of validation of building energy analysis tools and in particular efforts to validate the computer programs BLAST, DEROB, DOE, and SUNCAT. First, we discuss the nature of and need for validation. Then we present the validation efforts performed for each code. Finally, we list suggestions for further validating building energy analysis computer programs.

\subsection{Nature of and Need for Validation}

Building energy analysis simulations are used to predict energy flows in buildings. This includes temperatures, envelope losses, system performance, and electrical loads. The building in question may be an existing structure, a modification of an existing structure, or a new design. The accuracy of prediction of the building's performance depends on three main factors:

- Accuracy of the input data

- Applicability of the tool to the building and climate being analyzed

- Ability of the tool to predict real building performance when given perfect input data [1].

The accuracy of the input data is an important factor. The weather data available for the prediction are generally historical data for a site other than that of the building being analyzed. The data do not perfectly reflect the microclimate at the building site. The accuracy of the building description is constrained by the level of detail incorporated in the analysis tool, the accuracy to which the building properties are known, and the user's skill, experience, and available time [2].

To be truly applicable to a specific problem, the tool should predict building performance for that specific type of building in that particular climate, or it should predict performance for a statistically significant set of buildings and climates [3]. However, it is rarely feasible to collect sufficient experimental data or to apply a given analysis tool to a sufficient number and range of test cases to achieve complete confidence for all situations. Therefore, engineering judgment is commonly used to select test cases that represent typical applications of the tool. Analysis tool developers agree that models that have been verified for a few climates can be used with some degree of confidence to predict performance in other climates. However, they have less confidence in extrapolating from a single-zone structure to a multi-zone structure, and even less in predicting the performance of one building type (e.g., an indirect-gain south-aperture storage wall) based on the results of another model (e.g., a south-aperture direct-gain wall) [4.5].

The validation effort described in this report investigates the ability of the simulations to predict real building performance when given accurate input data. The four computer codes selected were: BLAST [6], DOE [7], DEROB [8], and SUNCAT [9]. For each code, some validation effort has already taken place. The remainder of this section reviews those validation studies.

\subsection{Existing Validation Studies}

The BLAST program was compared with empirical results from a direct gain test cell, a thermally massive building, and two Army buildings that were the size of small commercial buildings. The direct gain tests [10] compared the hourly air temperature of the test cell to 
BLAST predictions. Two time periods were-compared: one in September, one in December. During the former, the average temperature difference was $0.4^{\circ} \mathrm{C}\left(0.7^{\circ} \mathrm{F}\right)$, during the latter $0.8^{\circ} \mathrm{C}$ $\left(1.4^{\circ} \mathrm{F}\right)$. The investigators considered these results quite good. However, they identified several sources of ambiguity:

- The split between beam and diffuse components of solar radiation was not measured.

- Results are a strong function of the assumed infiltration rate.

The questions remaining after such a test are:

1. How well do single-zone test cell results indicate results for full-scale buildings?

2. How is the accuracy with which air temperature is predicted related to the accuracy with which thermal loads are predicted?

3. How do we explain the discrepancies that exist? (With limited experimental data taken, code validators must speculate about the causes of differences between observed and predicted air temperatures.)

The thermally massive building [10] was a well-insulated structure constructed in a large environmental chamber at the U.S. National Bureau of Standards (NBS). It allowed the testing of BLAST to be extended to a full-scale system including internal gains but excluding solar radiation. Two tests were run: a cooling load test and a night ventilation test. In the cooling load test, the zone temperature was allowed to float during half the day and constrained to a cooling set point during the other half. In the night ventilation test, the temperature was always allowed to float. Exterior air was introduced into the zone when the exterior air temperature fell below the interior air temperature. Temperatures were measured for both tests and cooling loads were measured for the cooling load test. Agreement for this test was not as good as that for the direct gain test cell. The investigators attributed these discrepancies to lack of experimental information or input ambiguities.

The test of the two Army buildings differed substantially from the two previous tests [11]. The buildings were occupied and the energy load was measured in terms of hourly electricity use. Thus, internal gain and occupancy schedules, infiltration, and the performance of cooling system equipment had to be estimated. BLAST predicted electricity consumption to within $-10 \%$ for a one-week period. However, the hourly differences were larger. Furthermore, input refinements based on measured data led to increases in deviations in one case, indicating self-canceling errors in the previous case. Trying to predict the performance of the Army buildings is closer to the situation faced by a typical user and reflects more closely the accuracies that probably will be achieved using a computer code. However, the ambiguities introduced by building occupancy and cooling system performance precluded resolution of the discrepancies that were observed.

The DOE program has been tested against empirical results from numerous buildings $[2,12]$. DOE-2 hourly predictions for temperature and heating loads were compared to data taken from the high-mass building in the NBS environmental chambers. Agreement was qualitatively evaluated as quite good by Kerrisk et al. [12]. Heat extraction rate for a conventional wood frame NBS test house in Houston, Texas, also was qualitatively assessed as showing good agreement 
between predicted and measured values. Hourly air temperatures for a direct gain office and warehouse in Pecos, New Mexico (monitored by Los Alamos Sandia Laboratories [LASL]), were predicted. Agreement was not considered good; investigators observed temperature differences of up to $4^{\circ} \mathrm{C}\left(7.2^{\circ} \mathrm{F}\right)$. However, the office also contained a water wall, and they attributed the differences to unmodeled manual operation of vents between the water wall and the office space. Finally, results of predicted hourly air temperature versus observed hourly globe temperature for the Williamson house (adobe construction with exterior insulation, ground floor and clerestory windows, and a slab-on-grade floor) are presented. Considering the difference between globe and air temperature, agreement was again qualitatively evaluated as good by these investigators.

Additionally, seven commercial buildings [2], 10 schools [13], and one hotel [14] have been modeled using DOE-2. Monthly and yearly energy use were predicted and compared to results measured for each building. Generally, the predictions were within 10\%-15\% of the observed yearly values. However, the monthly energy consumption values differed by as much as $50 \%$ $100 \%$. The magnitude of the monthly differences suggests the need for further investigation.

Besides the comparisons with empirical data, BLAST and DOE simulations have been compared to each other [2]. Carroll [15] compared DOE-2, BLAST-2, and NBSLD [16] (developed by NBS) for a single-family residence under various conditions. The three codes compared quite closely for annual and monthly loads. The greatest differences $(-12 \%)$ occur in the most severe climate. DOE-2 consistently predicted lower loads (and a greater time lag in hourly temperature profiles) because standard ASHRAE weighting factors were used. The least massive standard factors seemed to result in too much thermal mass. Custom weighting factors have been incorporated into DOE-2 since that time [12].

The DEROB code has been tested against empirical results from seven test cells [17], a passively heated building [18] and two houses [19,20]. Each test cell represents a different passive solar strategy (e.g., direct gain, Trombe wall). Hourly air temperatures are presented with varying degrees of agreement between simulated and observed values. Again, the code validator is left to speculate about the differences that exist. The first house tested was the Bruce Hunn residence [19], a two-story Trombe wall structure containing a rock bin for storage. Hourly air and Trombe wall temperatures are reported for seven days between December 27 and January 2. The mean absolute difference for the air temperatures was $1^{\circ} \mathrm{C}\left(1.8^{\circ} \mathrm{F}\right)$. Agreement for the Trombe wall was not as close, and the modeled temperature variation was less than what was observed.

The second house tested was the Williamson House, a direct gain passive solar structure located in Santa Fe, New Mexico. Hourly temperature profiles for interior air, the clerestory storage wall, the living room floor, and an interior wall were compared over a five-day period, December 26-30. The average difference for the air temperature was $1.8^{\circ} \mathrm{C}\left(3^{\circ} \mathrm{F}\right)$ with greater differences for wall and floor temperatures. Note that globe temperature was measured and compared to the air temperature predicted by the code. In addition to comparisons with empirical data, DEROB was tested against the TRNSYS energy analysis code [21]. A conventional residence and a small commercial building were studied. Thermal loads for one winter and one summer week were calculated for the residence. The observed and calculated loads differed by approximately $10 \%$. 
However, the hourly load variations differed significantly. Yearly loads were calculated for the light commercial building and differed by approximately $10 \%$.

The SUNCAT code has been tested against empirical results from a direct gain test cell [22]. Hourly values for air temperatures were compared for the six-month period November 1978 through April 1979. Root mean square (RMS) differences between hourly modeled and measured temperatures were approximately $1.1^{\circ} \mathrm{C}\left(2^{\circ} \mathrm{F}\right)$, indicating good agreement over the sixmonth period.

In summary, the accuracy with which a building's energy performance can be predicted using these particular tools depends on the accuracy of the input data. Using the most accurate input data they can obtain, knowledgeable users (usually the code authors) are generally able to predict hourly temperature variations. However, in most of these studies it is not possible for the reader to determine if this agreement is because of the assumptions made for unknown inputs or the accuracy of the codes. Building energy consumption has usually been predicted with accuracies no better than $10 \%$ and with errors as large as $50 \%$.

\subsection{Suggestions for Further Validation}

The following situations require further investigation:

- Code-to-code comparisons have been performed, but few comparisons have involved strongly solar-driven buildings with considerable mass storage.

- Single-zone test cell results have been used extensively, but few code developers have confidence in the extrapolation from single- to multiple zone predictions.

- Comparisons with empirical data raise some questions. However, these questions cannot be resolved because of insufficient data, and little effort has been directed toward performing follow-up experiments to investigate observed differences.

- Most previous studies have not measured input parameters sufficiently to eliminate significant input uncertainty. Additionally the process by which input values were obtained is generally not well documented.

- Although validation studies have been performed, no overall validation methodology has been developed.

The work discussed in the remainder of this report addresses these issues.

\section{$2.4 \quad$ References}

1. Black, A.W. 1978. "Is Bigger Really Better? A Heretical View of Computer Energy Programs.” ASHRAE Journal, January.

2. Diamond, S.C., Hunn, B.D., and Cappiello, CC. 1981. DOE-2 Verification Project. Phase I Interim Report. Los Alamos, NM: Los Alamos Scientific Laboratory, Report No. LA-8295-MS.

3. Cohen, J.S. 1980. Statistical Problems in Design Technique Validation. Golden, CO: Solar Energy Research Institute, SERI/RR-721-377. 
4. Bickel/CM, Inc. 1980. Performance Data for Passive Systems. A Proposed Data Format. Golden, CO: Solar Energy Research Institute, SERI/TR-0924-1.

5. Balcomb, J.D. 1978. "Simulation of Passive Solar Buildings." In Passive Solar: State of the Art, Vol. II, pp. 411-430.

6. Hittle, D.C. 1979. The Buildings Loads Analysis and System Thermodynamics Program, Version 2.0 Users Manual, Vols. I and II. Champaign, IL: U.S. Army Construction Engineering Research Laboratory.

7. DOE-2 Users Guide Version 2.1, Report \#LBL-8689, Rev. 1, May 1980.

8. Arumi, F.N., et al., The DEROB III System: Users Manual Vols. I and II, Austin, TX: Solenco.

9. Palmiter, L. and Wheeling, T. 1979. SUNCAT 2.4 User Notes. Butte, MT: Center for Appropriate Technology.

10. Bauman, F., Anderson, B., Carroll, W., Kammerud, R., and Friedman, N.E. 1981. Verification of BLAST by Comparison with Measurements of a Solar-Dominated Test Cell and a Thermally Massive Building, proceedings of the ASME Solar Energy Division Third Annual Conference on Systems Simulation and Economic Analysis/ Solar Heating and Cooling Operational Results. Berkeley, CA: Lawrence Berkeley Laboratory.

11. Herron, D. et al. 1980. Comparison of Building Loads Analysis and System Thermodynamics (BLAST) Computer Program Simulations and Measured Energy Use for Army Buildings. Report AD/A-085 573. Champaign, IL: Construction Engineering Research Laboratory.

12. Kerrisk, J. F., Moore, J.E., Schnurr, N.M., and Hunn, B.D. 1980. "Passive Solar Design Calculations with the DOE-2 Computer Program," The 5th National Passive Solar Conference, Proceedings of the International Solar Energy Society, October.

13. Sigworth, H.W., et al. 1978. DOE-1 Simulations of Ten Elementary Schools Base Case Reports. Report No. 8449. Berkeley, CA: Lawrence Berkeley Laboratory.

14. Griffin, T.M. 1979. A Comparison of DOE-2 Simulation Results and Actual Energy Data for the Marriott Hotel Boston, Massachusetts, W.S. Fleming and Associates Inc., report to the Marriott Corporation.

15. Carroll, W.L. 1979. Annual Heating and Cooling Requirements and Design Day Performance for a Residential Model in Six Climates: A Comparison of NBSLD, BLAST2 and DOE-2. Draft Report LBL-9270. Berkeley, CA: Lawrence Berkeley Laboratory.

16. Kusuda, T., 1976. NBSLD, The Computer Program for Solar Heating and Cooling Loads in Buildings, U.S. National Bureau of Standards, Building Science Series 69.

17. Arumi, F.N. 1979. "Field Validation of the DEROB/Pasole System," Proceedings of the Third National Passive Solar Conference, San Jose, CA.

18. Arumi, F.N. and Northrup, D.O. 1978. A Field Evaluation of the Thermal Performance of a Passively Heated Building as Simulated by the DEROB System, System Simulation and Economic Analysis for Solar Heating and Cooling. San Diego, CA: U.S. Department of Energy. 
19. Arum, F.N. et al. 1980. Field Validation of the DEROB System: The Bruce Hunn Residence. System Simulation and Economic Analysis Conference, San Diego, CA.

20. Wysocki, M.D. et al. 1980. "The Williamson House as Simulated by the DEROB System: A Field Validation," Proceedings of the 1980 Annual Meeting, American Section of the International Solar Energy Society, Phoenix, AZ.

21. TRNSYS, A Transient Simulation Program. 1979 Report 38-10, Engineering Experiment Station, University of Wisconsin, Madison, WI.

22. Palmiter, L. et al. 1979. "Summary of Passive Test Unit Performance," Proceedings of the $4^{\text {th }}$ National Passive Solar Conference, Kansas City, MO.

23. 1977 ASHRAE Handbook of Fundamentals. NY: American Society of Heating, Refrigerating and Air Conditioning Engineers, Inc.

24. Walton, G.N. 1980. "A New Algorithm for Radiant Interchange in Room Loads Calculations," ASHRAE Transactions 1980, Vol. 86, Pt. 2.

25. Lokmanhekim, M. 1975. Procedure for Determining Heating and Cooling Loads for Computerizing Energy Calculations, ASHRAE Task Group on Energy Requirements for Heating and Cooling of Buildings. NY: American Society of Heating, Refrigerating and Air Conditioning Engineers, Inc.

26. Schnurr, N.M. et al. 1979. "Applications of DOE-2 to Direct-Gain Passive Solar Systems: Implementation of a Weighting-Factor Calculative Technique," Proceedings of the 4th National Passive Solar Conference, Vol. 4, pp. 182-186. 


\subsection{Validation Philosophy}

In this work the overall validation effort contains three different kinds of tests: (1) code-to-code comparisons, (2) analytical verification, and (3) empirical validation. The philosophy and methodology for each individual test type are described in Sections 4.0, 5.0, and 6.0, respectively. In this section we outline the philosophy behind the overall methodology and explain the need for these three different kinds of tests.

\subsection{Validation Levels}

There are many levels of validation depending on the degree of control exercised over the possible sources of error in a simulation. There are seven sources of error:

1. Differences between the actual weather surrounding the building and the statistical weather input used with BEAS

2. Differences between the actual effect of occupant behavior and those effects assumed by the user

3. User error in deriving building input files

4. Differences between the actual thermal and physical properties of the building and those input by the user (generally from handbook values)

5. Differences between the actual thermal transfer mechanisms operative in individual components and the algorithmic representation of those mechanisms in BEAS

6. Differences between heat transfer mechanisms describing interactions between components and their representation in BEAS

7. Coding errors.

At the most basic level, the actual long-term energy usage of a building is compared to that calculated by the computer program with no attempt to eliminate sources of discrepancy. This level is similar to actual use of BEAS and, therefore, is favored by many representatives of the building industry. However, it is difficult to interpret the results of this kind of validation exercise because all possible error sources are operating simultaneously. Even if good agreement is obtained between measured and calculated performance, the possibility of offsetting errors prevents drawing conclusions about the accuracy of the method. More informative levels of validation are achieved by controlling or eliminating various combinations of error types. At the most detailed level, all known sources of error are controlled to identify and quantify unknown error sources.

Error sources 1 through 4 are external since they are independent of the internal workings of the method of calculation. Error sources 5 through 7 are internal and are directly linked to the internal workings of a prediction technique. Thus far in our work we have placed the highest priority on identifying internal error sources by carefully controlling external errors, since this area has not been well covered in previous research. 


\subsubsection{External Errors}

Although external errors may be a large source of discrepancy, they do not necessarily negate the usefulness of BEAS as a design or analysis tool. Weather data used by BEAS may differ somewhat from the particular microclimate surrounding a building, adversely affecting the prediction of building energy consumption. However, comparisons of the relative difference in energy consumption between design alternatives remain useful despite our imperfect characterization of climatic factors. In empirical validation studies, this uncertainty can be removed by recording at the building those weather data used by BEAS.

Occupant behavior can introduce large discrepancies between actual and predicted building performance. However, if internal errors are eliminated, the simulations allow us to quantify the effects of various occupant behavior patterns. This information could lead to guidelines to better inform and motivate occupants to operate their buildings optimally, or components that control the building automatically. The effect of occupants in smaller buildings can be eliminated from validation studies by using unoccupied buildings. This is not economically feasible in large commercial buildings. However, in such buildings the great number of occupants may tend to cancel the random effect of any single occupant.

User input errors can overpower all other effects in a simulation. As the user becomes more experienced at preparing input files, these errors become less common. Nevertheless, an excellent area for investigation is the effect of different user interfaces on speed, ease, and accuracy of input. (These effects are discussed further in Section 7.0.) In validation studies, these effects can be minimized by having several experienced users independently prepare input files from the same information. The files are then compared until unanimous agreement is reached.

Imperfect representation of the building's thermal properties usually has less effect on simulation accuracy than errors 1, 2, and 3. Ordinarily the practicing architect or engineer should find handbook values adequate for comparing the relative performance of design alternatives. In validation studies, however, use of accurate thermal properties becomes important. Both destructive and nondestructive testing procedures may be used to determine the actual thermal properties of the building (see Section 6.0 for further discussion). This is especially important when the validation study is attempting to isolate internal errors.

\subsubsection{Internal Errors}

We have seen in the previous section that successively more sophisticated levels of validation may be achieved by eliminating external error sources. Once all external sources are controlled, it is then possible to investigate internal error sources. Here again, the process of successive elimination may be applied.

Internal errors are more readily understood if we break the simulation process into two parts, modeling and numerical solution (see Figure 3-1). Modeling implies a simplification of the real physical processes at play in real buildings. These simplifications are made by code authors through the use of assumptions that allow the problem to be solved more easily within practical constraints [1]. These constraints may be related to such things as the core capacity and run-time of computer hardware or the availability of generally accepted mathematical expressions for certain physical processes. Two examples of such simplifying assumptions are the one- 
dimensionality of wall conductance, or the representation of interior infrared exchange as a constant increase to surface-to-zone convective film coefficients. These assumptions will influence the accuracy of a simulation depending on the building being analyzed. A small test cell with a relatively large ratio of "corner condition" would be more affected by the onedimensional wall conduction assumption than would a large building. A room with large temperature differences on different interior wall surfaces would be more affected by the lack of an infrared radiation network than a room with small temperature differences. Table 3-1 lists the effects of the constraints and assumptions common to BEAS.
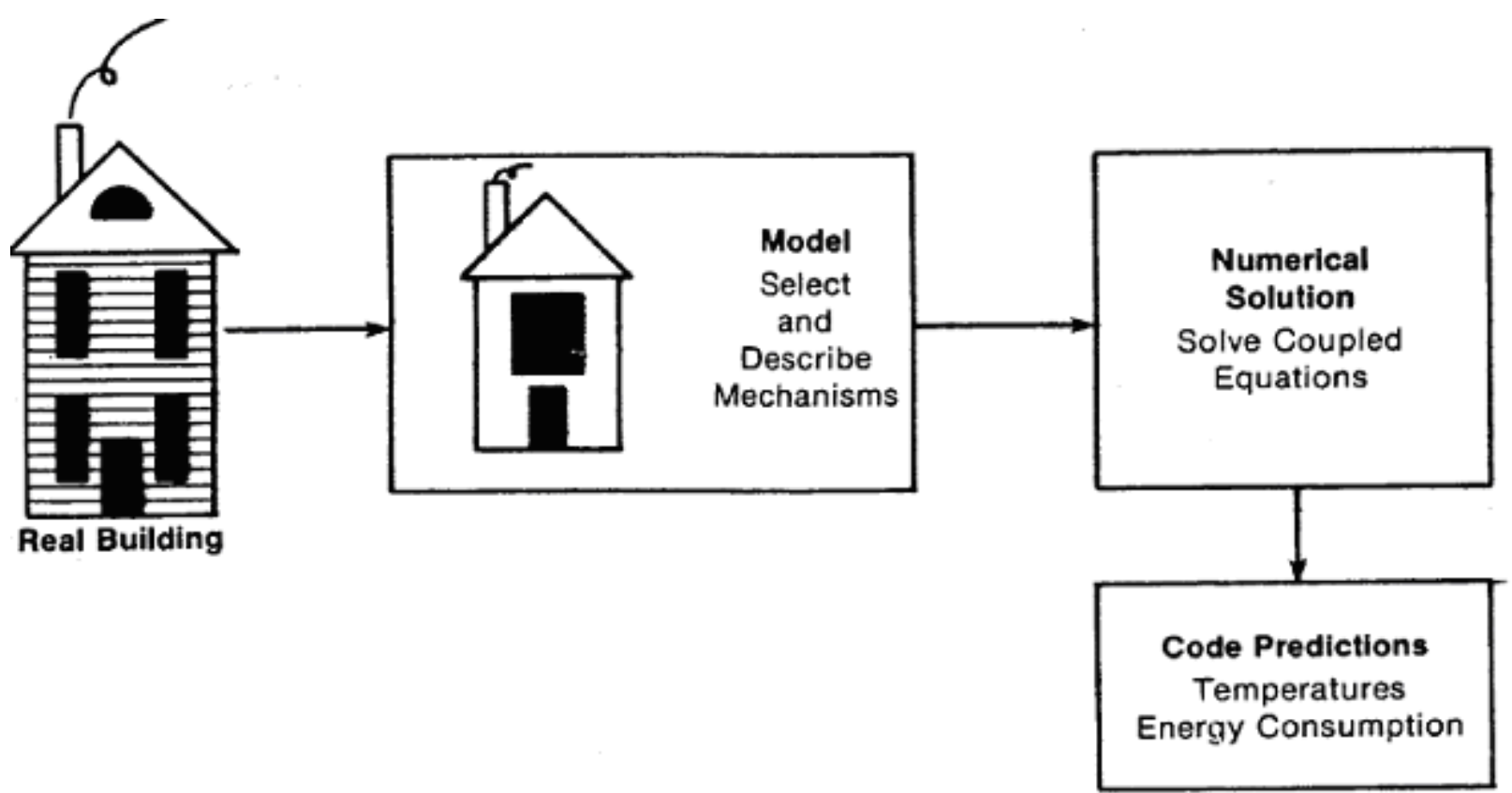

Figure 3-1. Simulation process

The second step of the simulation process is the numerical solution of the model. A model may lend itself to more than one numerical solution approach. For example, either transfer functions or finite differencing may be used to solve for one-dimensional wall conduction. The code author selects the numerical solution based on both objective and subjective criteria.

Differences between measured and calculated results can be due to either the model used or the numerical solution technique applied or both.

Internal error sources 5 and 6 each have a modeling component and a numerical solution component. Error source 5 refers to the modeling and numerical solution of individual heat transfer mechanisms, and error source 6 refers to the modeling and numerical solution of coupled mechanisms. Error source 7 is self-explanatory. 
Table 3-1. Effects of Constraints and Assumptions Common to BEAS

\begin{tabular}{|c|c|c|}
\hline Constraint & Assumption & Implications for Users \\
\hline $\begin{array}{l}\text { Computer time and } \\
\text { hardware are limiting } \\
\text { - 3-D conduction } \\
\text { - Infrared coupling } \\
\text { - Solar distribution } \\
\text { - Diffuse sky radiation }\end{array}$ & $\begin{array}{l}\text { Model can be developed to fit these } \\
\text { limitations }\end{array}$ & $\begin{array}{l}\text { Mechanisms simplified or } \\
\text { missing }\end{array}$ \\
\hline $\begin{array}{l}\text { Physical processes not fully } \\
\text { understood } \\
\text { - Advection } \\
\text { - Infiltration } \\
\text { - Ground coupling } \\
\text { - Night sky coupling }\end{array}$ & $\begin{array}{l}\text { Process is modeled as state of the } \\
\text { art will allow }\end{array}$ & $\begin{array}{l}\text { Users must determine } \\
\text { which processes are } \\
\text { important in their building } \\
\text { design and whether a } \\
\text { particular code is applicable }\end{array}$ \\
\hline $\begin{array}{l}\text { Difference between } \\
\text { published and installed } \\
\text { material properties }\end{array}$ & $\begin{array}{l}\text { Published data are good enough if } \\
\text { measured data are not available }\end{array}$ & $\begin{array}{l}\text { Can cause errors, but user } \\
\text { generally has no means to } \\
\text { make the necessary } \\
\text { measurements }\end{array}$ \\
\hline $\begin{array}{l}\text { User would prefer to not } \\
\text { look up material properties }\end{array}$ & $\begin{array}{l}\text { Program library contains } \\
\text { representative material properties }\end{array}$ & $\begin{array}{l}\text { When possible, user should } \\
\text { check material properties } \\
\text { against another source }\end{array}$ \\
\hline Information not in plans & $\begin{array}{l}\text { User uses judgment to develop } \\
\text { input files }\end{array}$ & -- \\
\hline Various & $\begin{array}{l}\text { Nondefinable, user-controlled } \\
\text { inputs, such as infiltration rates and } \\
\text { ground temperatures }\end{array}$ & $\begin{array}{l}\text { User can manipulate inputs } \\
\text { to produce desired results; } \\
\text { all inputs should have some } \\
\text { objective basis }\end{array}$ \\
\hline Description of microclimate & $\begin{array}{l}\text { External climate represented by } \\
\text { weather data }\end{array}$ & $\begin{array}{l}\text { User must judge the effects } \\
\text { of differences between the } \\
\text { weather data input and the } \\
\text { microclimate around the } \\
\text { building location }\end{array}$ \\
\hline Various & $\begin{array}{l}\text { Schedules can be neglected and } \\
\text { load or gain patterns can be } \\
\text { modeled by using constant, } \\
\text { average values }\end{array}$ & $\begin{array}{l}\text { Can cause errors, } \\
\text { particularly in commercial } \\
\text { building applications }\end{array}$ \\
\hline $\begin{array}{l}\text { Structure of the model used } \\
\text { in the simulation code }\end{array}$ & $\begin{array}{l}\text { Buildings can be fit into a model } \\
\text { with certain zoning limitations }\end{array}$ & $\begin{array}{l}\text { Some codes cannot be } \\
\text { used to represent the real } \\
\text { zoning of certain building } \\
\text { designs, limiting the } \\
\text { applicability of these codes }\end{array}$ \\
\hline $\begin{array}{l}\text { Knowledge about } \\
\text { occupancy effects }\end{array}$ & $\begin{array}{l}\text { Occupancy effects can be simply } \\
\text { modeled }\end{array}$ & $\begin{array}{l}\text { These effects may } \\
\text { dominate the performance } \\
\text { of a building, and the user } \\
\text { must be sure that the } \\
\text { assumptions in the model } \\
\text { are reasonable }\end{array}$ \\
\hline
\end{tabular}

At the most basic level, internal validation in the past has involved comparing measured longand short-term building performance data to simulated data. The long-term performance data generally show total energy usage for a period several times longer than the time constant of the building. This is to allow initialization effects to stabilize. Short-term performance data usually show average hourly temperature and hourly integrated energy fluxes. These data depend 
somewhat on the code solution technique and output capabilities. In either case, system level data is inconclusive because at this level of validation, many algorithms in each program are exercised simultaneously. As with external errors, if the calculated and measured performances disagree significantly, we cannot determine the internal source of disagreement. If close agreement is obtained, we cannot ascertain whether this is due to offsetting internal errors. We must therefore find ways to control, measure, or eliminate simultaneous internal errors, through detailed instrumentation of the building and by selecting relatively simple buildings.

A building energy analysis simulation code contains literally hundreds of variables, parameters, and algorithms. Ideally, validating an entire program would involve testing each algorithm in isolation and combination at some reasonable maximum and minimum value for each parameter. This would be impossibly expensive and time consuming. Even instrumenting a single building to the level necessary for one empirical validation test is a lengthy and expensive process. Therefore, validation test cases should be selected with great care, and the validation tests should be sequenced to obtain the most information from the least expenditure of time and resources.

One way to do this is to look at the structure of the simulations themselves. Most of them are divided into distinct blocks for modeling loads, systems, and plant. The loads portion determines the skin load on the building. The systems portion deals with controls and distribution systems. The plant portion models the primary boilers, furnaces, or chillers that power the system. The loads portion of a simulation has the most general effect on all other portions of the program. If a particular algorithm for a piece of equipment is faulty, this will not affect the final results unless that piece of equipment is used in the simulation. However, if a basic heat transfer algorithm is faulty in the loads block, all results obtained with the program will be faulty regardless of the accuracy of the rest of the program. For this reason, our approach has been to focus first on the ability of BEAS to correctly calculate envelope loads.

\subsubsection{Extrapolations}

Because of the great number of parameters in any BEAS, five extrapolations are frequently made in validation studies as shown in Table 3-2 and described in the following sections.

Table 3-2. Extrapolations Made in Validation Studies

\begin{tabular}{|rl|l|}
\hline \multicolumn{1}{|c|}{ Obtainable Data Points } & \multicolumn{1}{c|}{ Extrapolation } \\
\hline 1. A few climates & Many climates \\
\hline 2. Short-term (monthly) total energy usage & Long-term (yearly) total energy usage \\
\hline 3. Short-term (hourly) temperatures or fluxes & $\begin{array}{l}\text { Long-term (yearly) total energy usage and } \\
\text { temperature extremes }\end{array}$ \\
\hline 4. $\begin{array}{l}\text { A few buildings representing a few sets of } \\
\text { variable mixes }\end{array}$ & $\begin{array}{l}\text { Many buildings representing many sets of variable } \\
\text { mixes }\end{array}$ \\
\hline 5. $\begin{array}{l}\text { Small-scale, simple test cells and } \\
\text { buildings }\end{array}$ & Large-scale complex buildings \\
\hline
\end{tabular}

\subsubsection{Extrapolation No. 1}

Since testing under every weather condition is impossible, a few exemplary climates must be selected to stress different heat transfer mechanisms. Testing in one climate can easily conceal 
large errors in a code, so two or more very different climates should be used. This kind of concealed error was revealed in a SERI comparative study (Section 4.0) where a simple directgain building was modeled. Using Madison, Wisconsin, TMY weather data, we found BEAS agreed within $\pm 0.5 \%$. When we used Albuquerque, New Mexico, TMY weather data, the codes disagreed by as much as $50 \%$.

\subsubsection{Extrapolation No. 2}

Long-term (yearly) tests are generally impractical, so most parameters must be tested in the short term. Based on the short-term accuracy of the program (usually a week to a month), the extrapolation must be made to the yearly accuracy of the program. Unfortunately, short-term tests tend not to reveal small additive errors, and only certain heat transfer mechanisms may be stressed by the particular short-term weather sequence at the time of the test.

\subsubsection{Extrapolation No. 3}

It is difficult to tell what effect short-term temperature discrepancies will have on the accuracy of long-term energy usage prediction. The relationship between hourly temperature measurements and yearly energy prediction in part depends on other parameters in the simulation such as the thermostat control strategy used. For example, if the upper or lower limit of a dead band, frequently falls between the extremes of predicted and actual temperature as in Figure 3-2a, nontrivial, total energy usage differences could occur even though predicted hourly temperatures were quite close. However, if predicted and actual temperatures are frequently within the dead band when they disagree, then the effect on total energy usage would be small even though the temperature differences were relatively large as in Figure 3-2b. In addition, even if the temperature is accurately predicted, the algorithms that calculate energy loads based on temperature could be faulty.

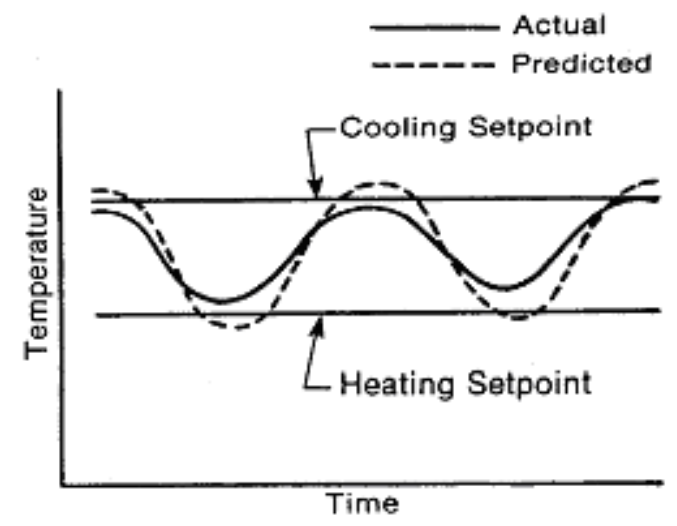

(a) Deadband limits between measured and calculated temperatures

Figure 3-2 Deadband limits

\subsubsection{Extrapolation No. 4}

Extrapolation No. 4 is unavoidable since it is impossible to test the simulation against every conceivable building that the simulation should properly handle. This would eliminate the need 
for the simulation itself, since we would already have data on every building that we could simulate. Therefore, we must select configurations that stress those heat-transfer mechanisms representative of most buildings. At SERI, we used a two-zone test cell as the simplest allowable configuration to ensure that the mechanisms and algorithms necessary for multi-zone analysis are tested. Additionally, we are using a full-scale, four-zone building to test the extrapolation from a two-zone, test-cell scale to multi-zone full scale.

\subsubsection{Extrapolation No. 5}

It would be prohibitively expensive to achieve the same level of instrumentation and control in a large commercial building as in a small residence. Therefore, it is necessary to extrapolate from the ability of the simulations to accurately calculate small building envelope loads to their ability to handle large building envelope loads. This is an acceptable extrapolation since small buildings are dominated more by skin loads than larger buildings are. However, in large buildings the accuracy of BEAS in modeling the systems and plant response needs to be checked in addition to the response to skin loads.

\subsection{Methodological Approach}

Each comparison between measured and calculated performance represents only a single data point in an immense $\mathrm{N}$-dimensional parameter space. We are constrained to establishing very few data points within this space. Yet, we must somehow be assured that the results at these points are not coincidental and are representative of the validity of the simulation elsewhere in the parameter space. Our validation approach is an attempt to minimize the uncertainty of the extrapolations we must make in any validation study by using three related techniques:

- Comparative studies

- Analytical verification

- Empirical validation.

These three approaches are shown schematically in Figure 3-3. Each approach focuses on different aspects of the validation problem. By integrating these approaches in the overall validation process, the advantages of each are enhanced and the disadvantages minimized.

\subsubsection{Comparative Studies}

A comparative study involves a direct comparison of the results obtained from two ${ }^{*}$ or more BEAS using equivalent input. The comparative study is a useful technique because it does not require data from a real building. Buildings can be created and placed in a real or imagined environment such that various heat transfer mechanisms are stressed as desired: The investigator has complete control over the accuracy of the input, and all external errors are easily eliminated. Comparisons may be sequenced to test the sensitivity of the simulations to various input modifications. Comparative studies quickly show if further, more detailed investigation is merited. A large number of different test cases can easily be run in a relatively short time. Proper sequencing of test cases indicates those portions of the simulation that should be investigated in detail. Test cases may be simpler than any real building, or as complex and realistic as needed.

\footnotetext{
* A code may be run against itself to quantify the effect of using one subroutine versus another. This is similar to a parametric study where one parameter is varied in the building to quantify the sensitivity to that parameter.
} 
Internal discrepancies may be investigated by defining test cases that successively eliminate or add various heat transfer mechanisms.

The great disadvantage of the comparative technique is the absence of a truth model. For this reason the comparative study is best done using BEAS with very different modeling and solution approaches. If several simulations based on similar modeling approaches agree, it is still quite possible that they are all incorrect. If several simulations based on completely different modeling approaches agree on a number of different test cases, it is likely that the physical systems are being well characterized.

Because of the lack of a truth model, the comparative technique is most powerful when used with the analytical and empirical techniques. The comparative technique may be used before empirical validation studies are done to identify the need for empirical validation and to define the level of empirical validation needed. For example, if most BEAS agree on analysis of conventional buildings but disagree on passive buildings, we design our empirical validation study to test passive building types. If the simulations always agree in the loads portion of the program but diverge when systems and plant are included, we then know to design our empirical validation study to include these factors.

The comparative technique is also useful after an empirical validation study has been completed since it allows us to extrapolate the results of the empirical validation test cases to other cases. For example, assume that we record data on a building in Golden, Colorado, and that after some corrections to BEAS we are able to obtain close agreement for both long- and short-term data with several of the simulations. We can quickly test the generality of these results by simulating the test building in several different climates. If close agreement between codes is still obtained, we can then vary the building to establish the range of building types within which the programs are validated. Any case in which the results diverge is useful for defining an empirical validation study if the cause of the divergence cannot be found through less expensive means. 


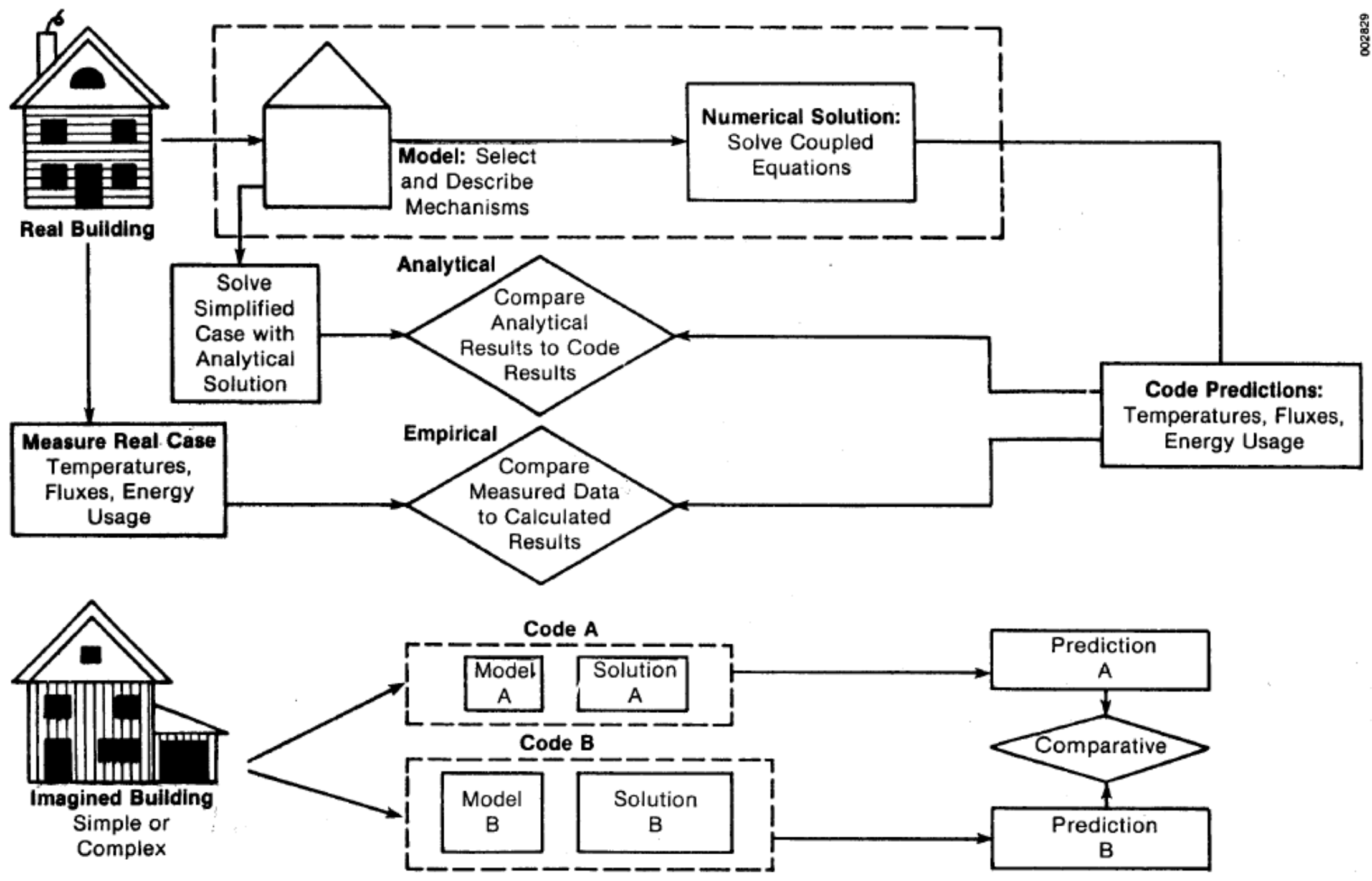

Figure 3-3. Analytical, empirical, and comparative techniques 


\subsubsection{Analytical Verification}

A typical building energy analysis simulation program contains hundreds of variables and parameters. The number of possible cases that can be simulated by varying each of these parameters in combination is astronomical and can never be fully tested. However, universal to the accurate calculation of any case are the fundamental heat-transfer mechanisms of conduction, radiation, and convection. It is possible to define simple test cases that can be solved analytically and that can also be simulated using BEAS. These cases can be defined to test those fundamental heat transfer mechanisms that, in isolation or limited combination, have the greatest impact on building thermal performance (see Section 4.0). These analytical test cases are much simpler than real buildings in that the boundary conditions are strictly controlled so they can be solved analytically. The power of this technique is that major errors in the thermal solution algorithms of a simulation may readily be identified and isolated. The analytical solution is the truth model, and all the uncertainty of simultaneous error sources is eliminated. The disadvantage of this technique is the limited number of configurations and combined mechanisms for which analytical solutions may be derived. Additionally, analytical verification can only test the correctness of the numerical solution portion of internal error sources 5 and 6 . It cannot test the correctness of the model itself. Even though simulation may pass all analytical tests, it may not be correct when used on real buildings. However, the power of analytical verification is increased when used with the comparative study technique. By starting with the very simple analytical test cases, baseline agreement may be established between different BEAS and the analytical solution. The test cases may then be modified one parameter at a time toward the more complex case of a real building. The point at which BEAS diverge indicates an area for investigation with empirical techniques. Additionally, the disagreement is easily quantified so that the value of a sophisticated and time-consuming algorithm may be assessed against that of a simplified approach.

\subsubsection{Empirical Validation}

In empirical validation a real building or test cell is instrumented and the calculated results from BEAS are compared to the measured results obtained from the instrumentation. ${ }^{\dagger}$ The comparison variables are uncertain because of direct measurement error. Measurement error also causes a degree of input uncertainty that when propagated through simulation leads to some output uncertainty. The total uncertainty consists of both these uncertainties. For purposes of validation, deviations between measured and calculated values significantly beyond these uncertainty bands are attributed to either the modeling or numerical solution component of error sources 5 and 6 or 7 , given that all inputs have been measured. (A method for quantifying these uncertainties is described in Section 6.0.)

For the more highly controlled levels of validation, an extremely detailed level of data acquisition is required. This level of data acquisition has been termed a class A system by investigators in the field [2]. This system requires approximately 200 sensors for relatively simple buildings, and the building must be unoccupied to control external error source 2 . There are currently very few class A sites in the United States. Table 3-3 lists the sites we have identified.

\footnotetext{
${ }^{\dagger}$ Proper instrumenting of buildings for validation is discussed Section 6.0.
} 
Table 3-3. Class A Validation Sites

\begin{tabular}{|l|l|c|l|}
\hline \multicolumn{1}{|c|}{ Site } & \multicolumn{1}{|c|}{ Location } & $\begin{array}{c}\text { Percentage } \\
\text { Class A }\end{array}$ & Status as of 5/1/83 \\
\hline SERI two-zone test cell & $\begin{array}{l}\text { SERI } \\
\text { Golden, Colorado }\end{array}$ & 100 & Taking data \\
\hline SERI four-zone residence & $\begin{array}{l}\text { SERI } \\
\text { Golden, Colorado }\end{array}$ & 100 & Taking data \\
\hline Reconfigurable test facility & $\begin{array}{l}\text { CSU } \\
\text { Fort Collins, Colorado }\end{array}$ & 100 & $\begin{array}{l}\text { Building constructed, } \\
\text { in preparation }\end{array}$ \\
\hline NBS & $\begin{array}{l}\text { NBS } \\
\text { Washington, D.C. }\end{array}$ & 90 & Taking data \\
\hline
\end{tabular}

Class B and C data acquisition systems have also been designed by SERI. A class B system allows about 20 channels of integrated or averaged hourly data collection. A class $\mathrm{C}$ system meters the total energy usage of the building on a monthly basis. Class $\mathrm{B}$ and $\mathrm{C}$ buildings are usually occupied.

Investigators must realize the distinction between the levels of validation possible with these three systems. Table 3-4 shows the error sources it is possible to isolate with the different systems.

Table 3-4. Degree of Control of Error Sources versus Level of Instrumentation

\begin{tabular}{|c|l|}
\hline Class & \multicolumn{1}{c|}{ Degree of Control } \\
\hline A & All error sources may be isolated. \\
\hline B & Error sources 1, 2, 4, 5, and 6 controlled. \\
\hline C & All error sources uncontrolled. \\
\hline
\end{tabular}

Class B systems could attain a higher level of control if instrumentation was included to separate direct beam and diffuse radiation. This would require a shadow banded pyranometer and a person to adjust the band frequently. For unoccupied class B sites with this modification, error sources 1 and 2 would be controllable. Class B data would then be useful with the comparative technique to reduce the uncertainty of extrapolations 1,2 , and 4.

Empirical validation is so time consuming and expensive that the empirical test cases must be chosen with great care. The analytical and comparative techniques provide information that help define and prioritize the empirical test cases. Once empirical data are collected, the comparative technique allows us to extrapolate to cases for which we have no empirical data.

Table 3-5 shows the advantages and disadvantages of each technique. When used separately, they fail to provide enough information for an overall validation. However, when used together, they form a powerful validation methodology. 
Table 3-5. Validation Techniques

\begin{tabular}{|c|c|c|}
\hline Technique & Advantages & Disadvantages \\
\hline $\begin{array}{l}\text { Comparative } \\
\text { Relative test of model } \\
\text { and solution process }\end{array}$ & $\begin{array}{l}\text { - No input uncertainty } \\
\text { - Any level of complexity } \\
\text { - Inexpensive } \\
\text { - Quick: many comparisons possible }\end{array}$ & No truth standard \\
\hline $\begin{array}{l}\text { Analytical } \\
\text { Test of numerical } \\
\text { solution }\end{array}$ & $\begin{array}{l}\text { - No input uncertainty } \\
\text { - Exact truth standard given the } \\
\text { simplicity of the model } \\
\text { - Inexpensive }\end{array}$ & $\begin{array}{l}\text { - No test of model } \\
\text { - Limited to cases for which } \\
\text { analytical solutions can be derived }\end{array}$ \\
\hline $\begin{array}{l}\text { Empirical } \\
\text { Test of model and } \\
\text { solution process }\end{array}$ & $\begin{array}{l}\text { - Approximate truth standard within } \\
\text { accuracy of measurements } \\
\text { - Any level of complexity }\end{array}$ & $\begin{array}{l}\text { - Measurement involves some } \\
\text { degree of input uncertainty } \\
\text { - Detailed measurements of high } \\
\text { quality are expensive and time } \\
\text { consuming } \\
\text { - A limited number of data sites are } \\
\text { economically practical }\end{array}$ \\
\hline
\end{tabular}

Figure 3-4 shows the flowchart that an independent code author would follow in applying the validation methodology. The first step is to run the code against the analytical test cases described in Section 5.0 of this report. This checks the numerical solution of the major heat transfer models in the code. If a discrepancy occurs, the source of the difference must be identified and corrected before any further validation is done. The next step is to run the code against class A empirical validation data and to correct discrepancies. (SERI is currently collecting these data and will make them available in future publications.) Step 3 involves checking the code against several prevalidated BEAS in a number of comparative studies. The prevalidated BEAS will have successfully passed steps 1 and 2 and will have shown substantial agreement for all the comparative study cases. These comparative study cases will, to the extent possible, use class B data. SERI will attempt to prevalidate the DOE, BLAST, and SERIRES programs as part of its class A empirical validation project and then run the programs in a number of comparative studies. These comparative studies will be defined to extrapolate the results obtained in the empirical validation studies to other locations and other building types. Figure 3-5 shows a flowchart for the SERI prevalidation activity. 


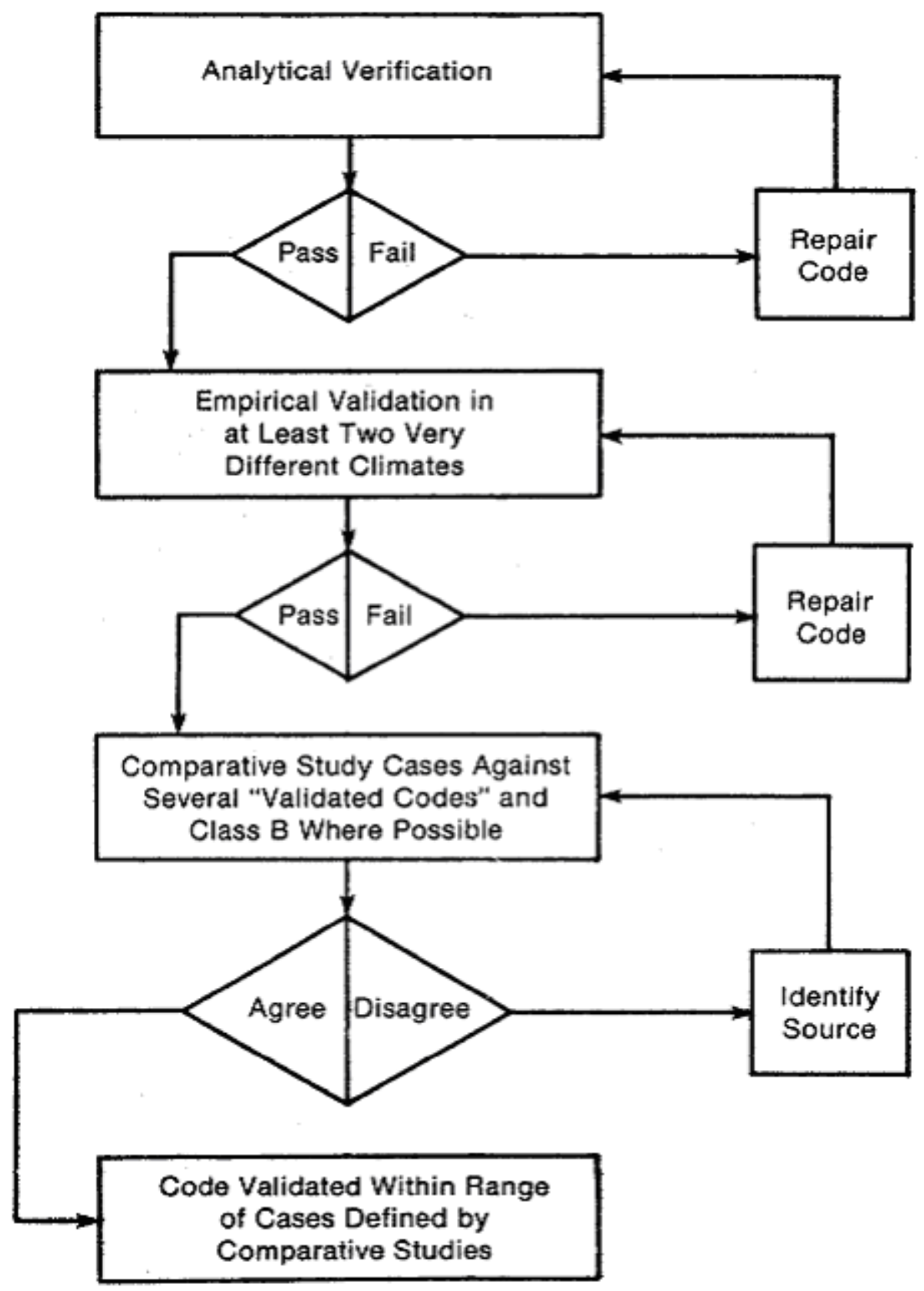

Figure 3-4. Flowchart for using SERI validation method

In Sections 1.0, 2.0, and 3.0, we described the historical, technical, and methodological context surrounding the SERI work. Sections 4.0, 5.0, and 6.0 describe the validation work accomplished in FY 1981. 


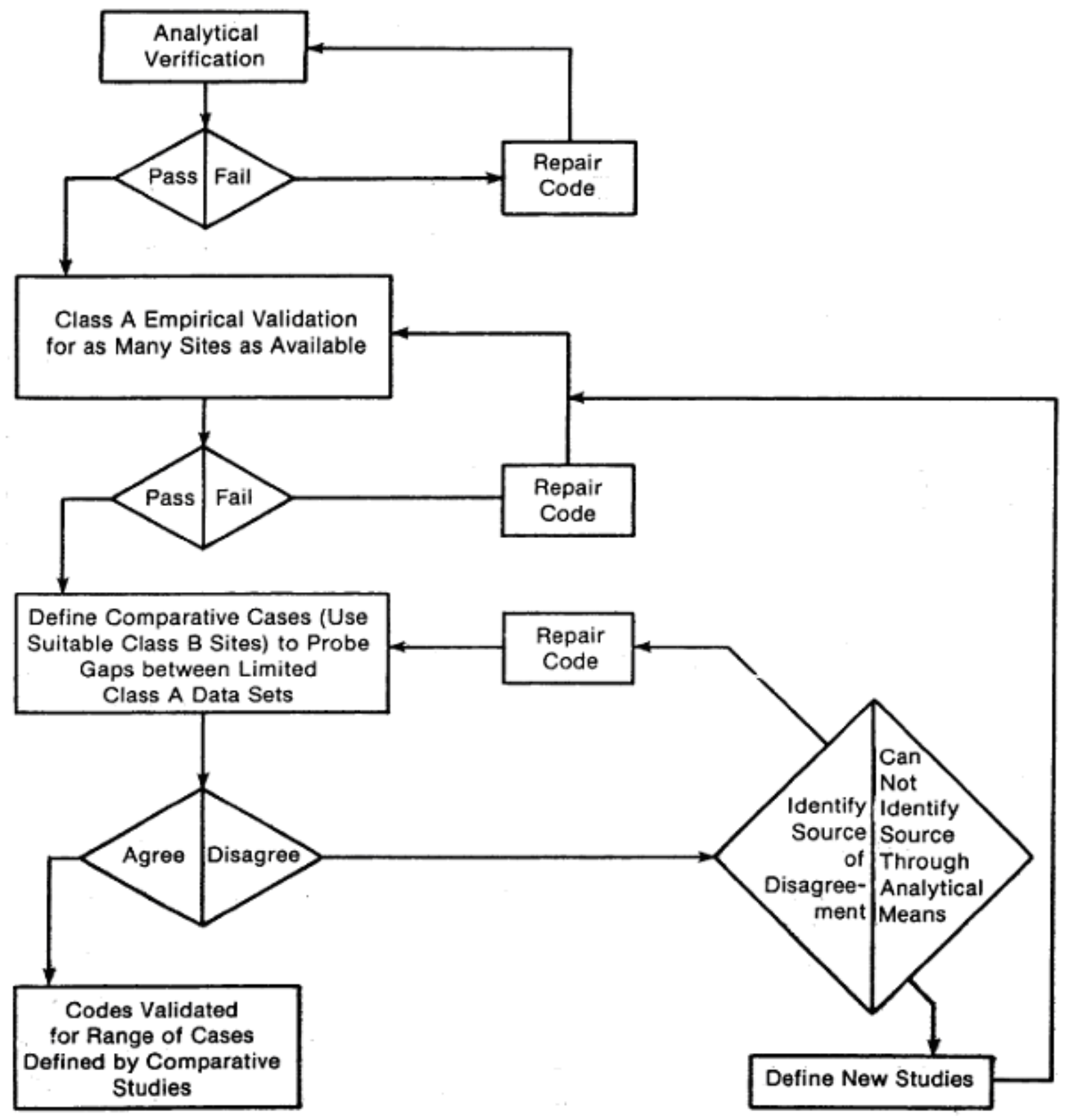

Figure 3-5. Flowchart of SERI prevalidation activities with DOE, BLAST, SUNCAT, and DEROB

\subsection{References}

1. Wortman, D. Assumptions, Accuracy, and Results of Building Energy Simulation Codes, SERI/TP-721-1331. Golden, CO: Solar Energy Research Institute, unpublished work.

2. McKinstry, M. et al. "System Performance Evaluation at the Class A Level Performance Evaluation of Passive/Hybrid Solar Heating and Cooling, Appendix B, PO-NQ-9-4059-1. Golden, CO: Solar Energy Research Institute, unpublished work. 


\subsection{Comparative Studies}

The comparative studies of selected BEAS programs were conducted in two phases [1,2]. In the first phase we compared DOE-2.1, BLAST-2MRT, DEROB-3, and SUNCAT-2.4 [3-8] by modeling a high- and low-mass direct-gain building in Madison, Wisconsin. The results of the Phase I study raised several questions that were further investigated in the Phase II comparisons. At the same time as the comparative studies were being conducted, an analytical verification technique was being developed [9] (the results are detailed in Section 5.0). However, some reference to the analytical results will be made in this section since chronologically they became available while the comparative studies were in progress.

\subsection{Phase I Comparative Study: DOE-2.1. BLAST-2MRT, DEROB-3, and SUNCAT-2.4}

A number of validation studies were discussed in Section 2.0. Many of these studies concluded that the simulations in question were essentially reliable and accurate. This comparative study was designed to check the credibility of these claims and to test our ability to use these complex programs correctly. To do this, we designed a very simple direct-gain building with a high- and a low-mass option. We kept the building simple so differences in results could be analyzed and equivalent input to the four programs ensured. The codes were compared on the basis of hourly temperature profiles and annual heating and cooling loads. In addition, the radiation processors and sky modeling algorithms were compared as originally coded and then standardized so incident radiation was equivalent for all four codes. The building is described in Figure 4-1 and Table 4-1.

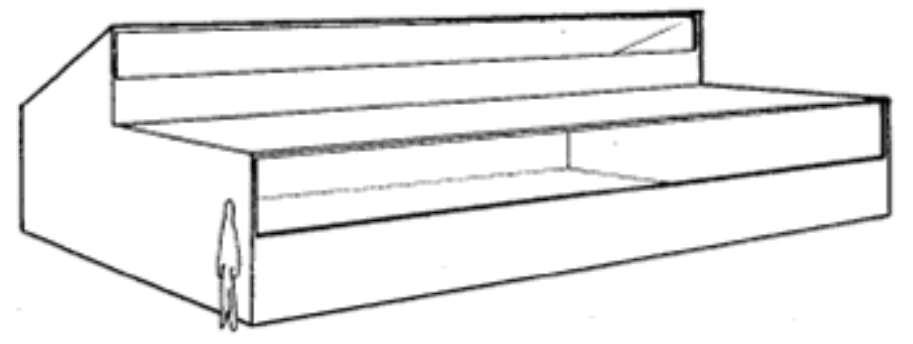

Figure 4-1. Comparative study test building

\subsubsection{Input Equivalence}

We put much effort toward ensuring input and model equivalence among the codes. We eliminated known sources of difference wherever possible so the results of the study would be useful whether or not the codes agreed. If codes agreed, the significance of the algorithmic differences between the codes could be analyzed by changing one parameter at a time. Since agreement was not obtained, we have a clear indication that further study is needed because known sources of disagreement were already eliminated. 
Table 4-1. Comparative Study Test Building Characteristics

\begin{tabular}{|l|}
\hline $43.1^{\circ} \mathrm{N}$ Latitude \\
\hline Madison, Wisconsin, TMY weather data \\
\hline $1500 \mathrm{ft}^{2}$ floor area \\
\hline $350 \mathrm{ft}^{2}$ glass area (double-glazed, vertical, due south) \\
\hline $300 \mathrm{Btu} / \mathrm{h}^{\circ} \mathrm{F}$ UA overall (includes infiltration but not glazing) \\
\hline $65^{\circ} \mathrm{F}-$ heating set point \\
\hline $75^{\circ} \mathrm{F}$ - cooling set point \\
\hline Low-mass case: 0.5 -in. gypsum board on all walls \\
\hline High-mass case: 8-in. concrete on all walls \\
\hline No shading, night insulation, or ground coupling \\
\hline Zero external absorptivity \\
\hline Single zone \\
\hline
\end{tabular}

The effort to ensure equivalence was complicated by three problems:

- A mechanism modeled in one code and not in another (e.g, external absorptivity of opaque surfaces)

- Mechanisms at different levels of rigor in the codes (e.g., internal radiation networks)

- Undocumented assumptions or mechanisms $1 n$ the codes (e.g., hardwired perimeter loss model).

In many instances we overcame these problems by either crippling a capability in a complex code to match a simpler code or by choosing a simpler building model. Where these alternatives were not possible, we conducted sensitivity studies to determine the potential range of error attributable to the input variable. We then used our best engineering judgment to minimize that range.

\subsubsection{Solar Radiation}

The original versions of the codes contained different solar radiation processors. Figure 4-2a,b shows the hourly radiation differences between the codes for a clear and cloudy winter day in Madison, Wisconsin. The largest difference is shown by the DOE-2.1 program, which uses an anisotropic model for sky diffuse radiation instead of the isotropic assumption used by the three other codes. Figure 4-3a,b shows the effect on annual heating and cooling loads of using an isotropic versus an anisotropic sky diffuse algorithm in the DOE-2.1 program. In Albuquerque, New Mexico, a predominantly clear climate, and Madison, a predominantly cloudy climate, we observed differences of approximately 10\%. To keep these differences from overpowering other effects, we standardized the solar radiation algorithms. Global horizontal and direct normal radiation read directly from the TMY tape were used to separate direct and diffuse radiation. We assumed an isotropic model to account for diffuse radiation. With this change, the final versions of all four codes showed only minor differences in incident radiation as shown in Fig. 4-4. These minor differences may be explained by variations in declination formulas, time step definitions, and solar versus local time assumptions. 


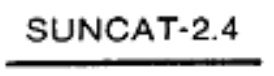

DEROB-III

DOE-2.1

ISOTROPIC

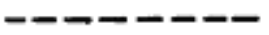

BLAST-3.0

DOE-2.1

ANISOTROPIC

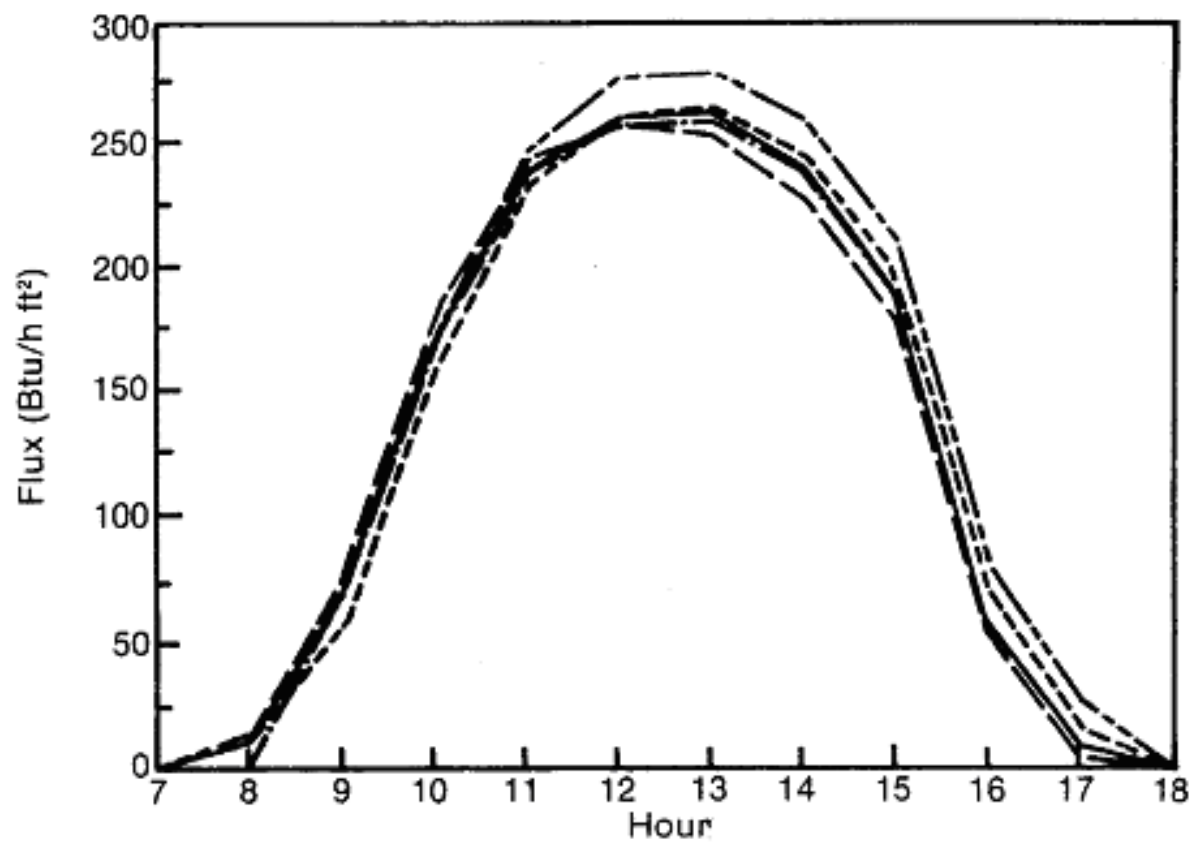

SUNCAT-2.4

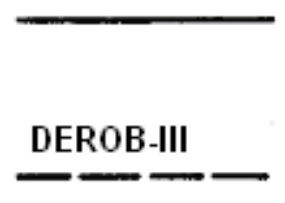

DOE-2.1

ISOTROPIC

BLAST-3.0

DOE-2.1

ANISOTROPIC

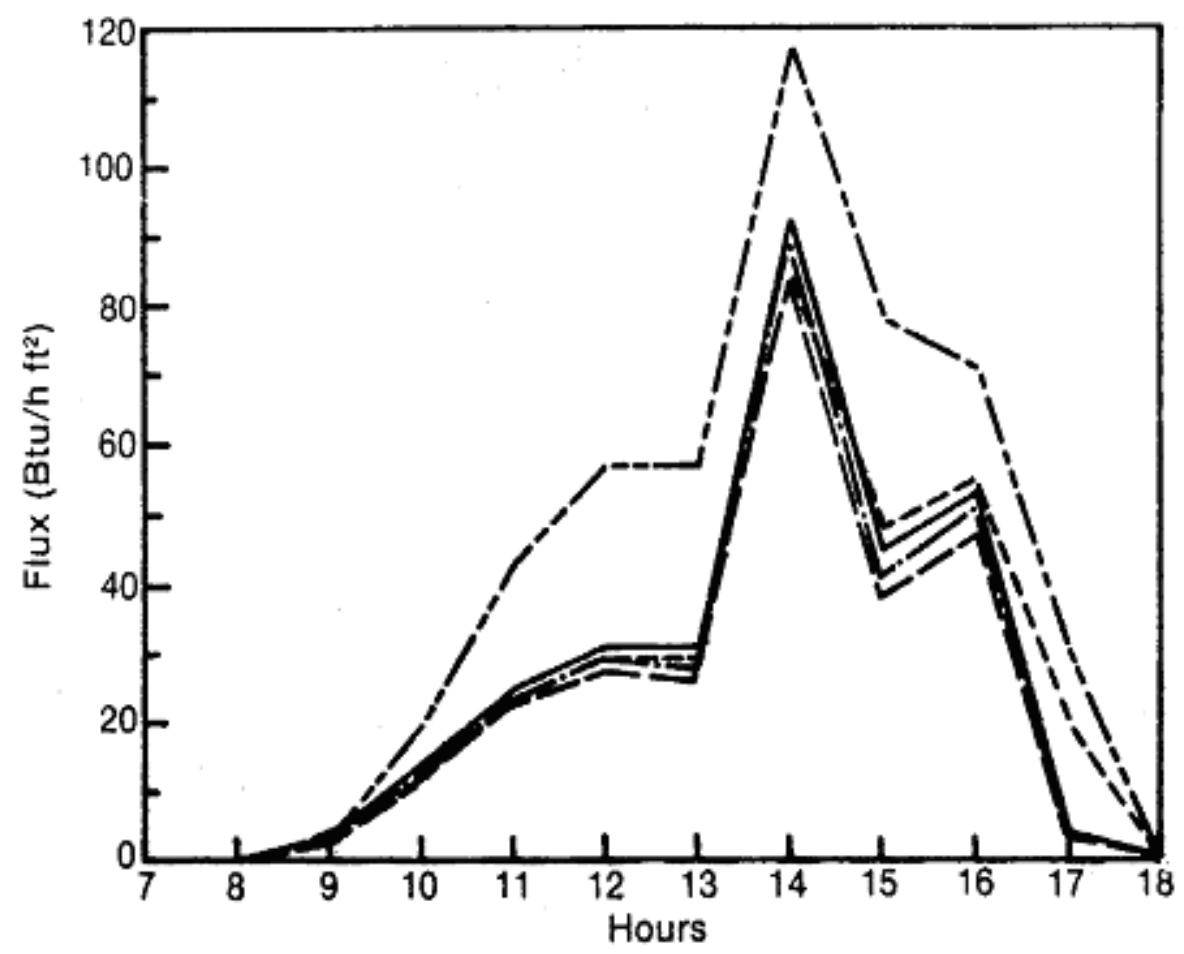

Figure 4-2. Incident radiation flux, Madison, Wisconsin 


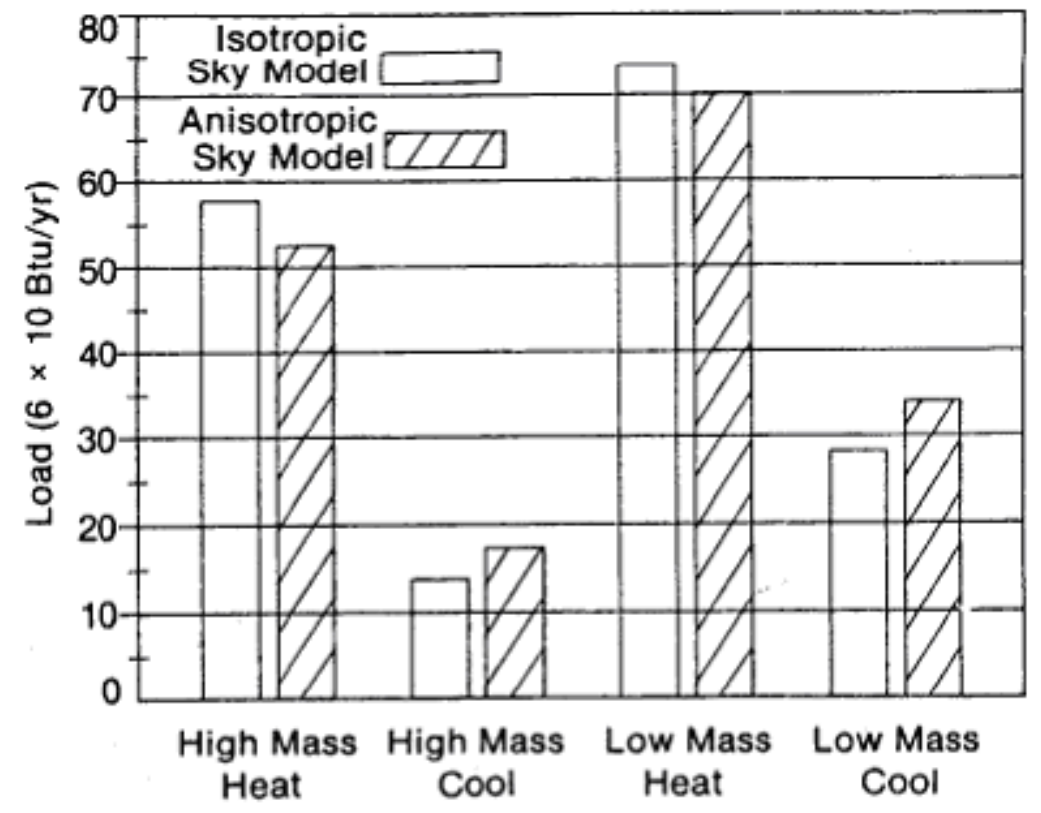

(a) Madison, Wisconsin

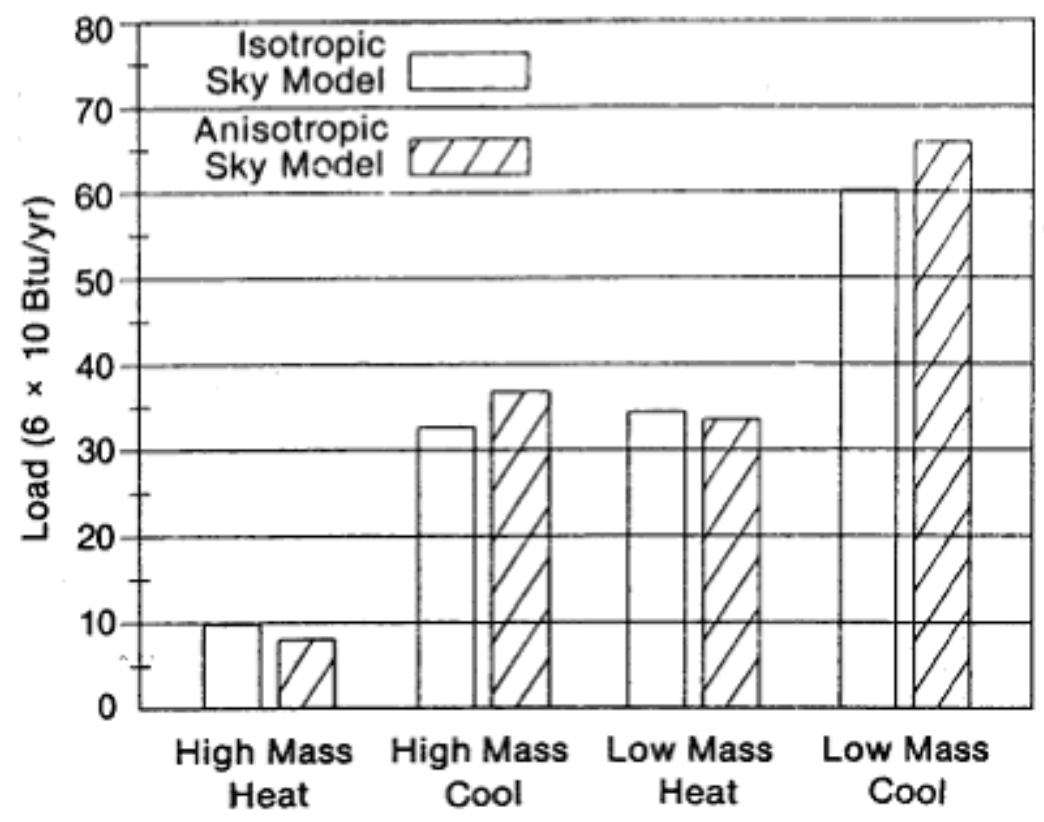

(b) Albuquerque, New Mexico

Figure 4-3. DOE 2.1 Isotropic versus anisotropic sky 


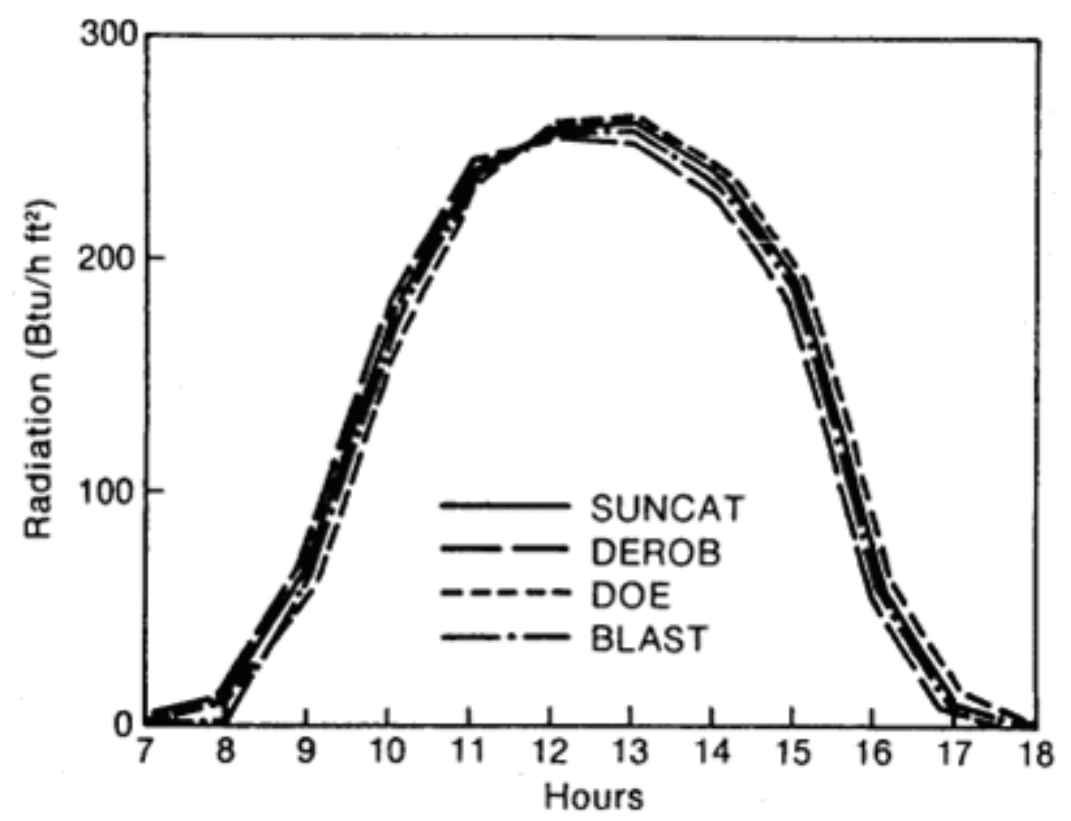

Figure 4-4. January 21 incident radiation for four codes

\subsubsection{Hourly Free-Float Temperature Profiles}

Figures 4-5 and 4-6 show hourly air temperature profiles for rooms in the low- and high-mass buildings, respectively, on January 21 . This was a typical, clear, cold day that was far enough from the beginning of the simulation to eliminate initialization effects.

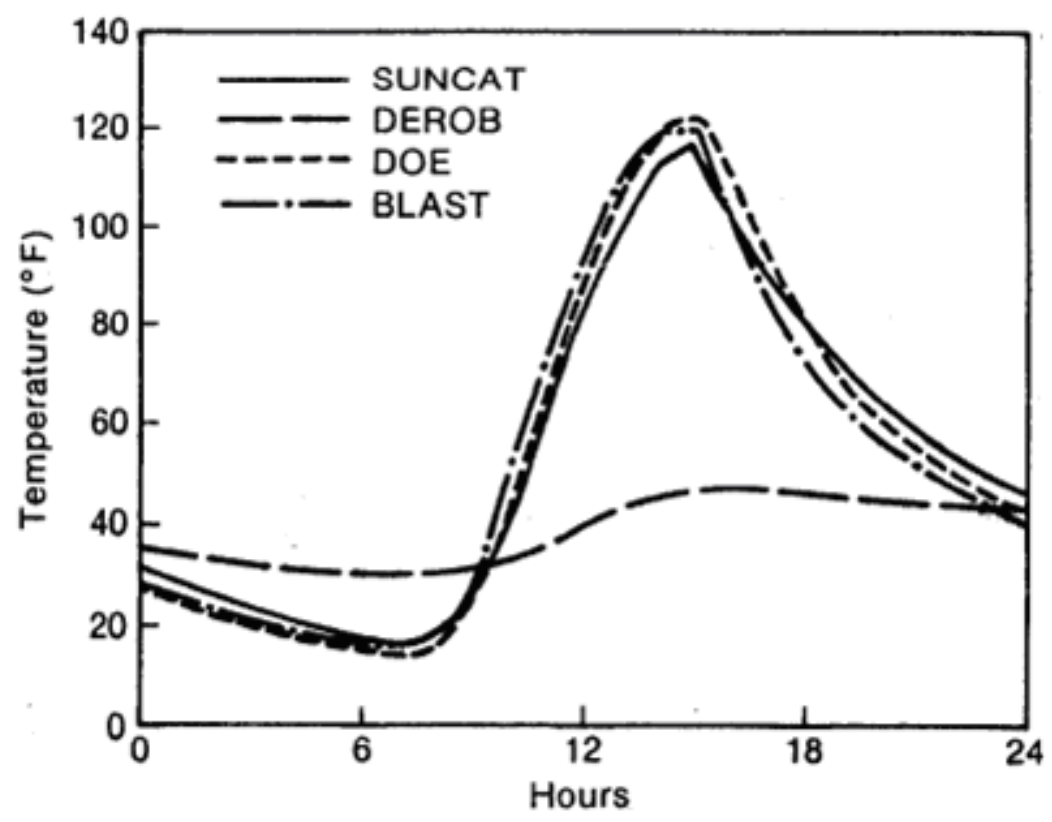

Figure 4-5. January 21 high-mass room air temperature 


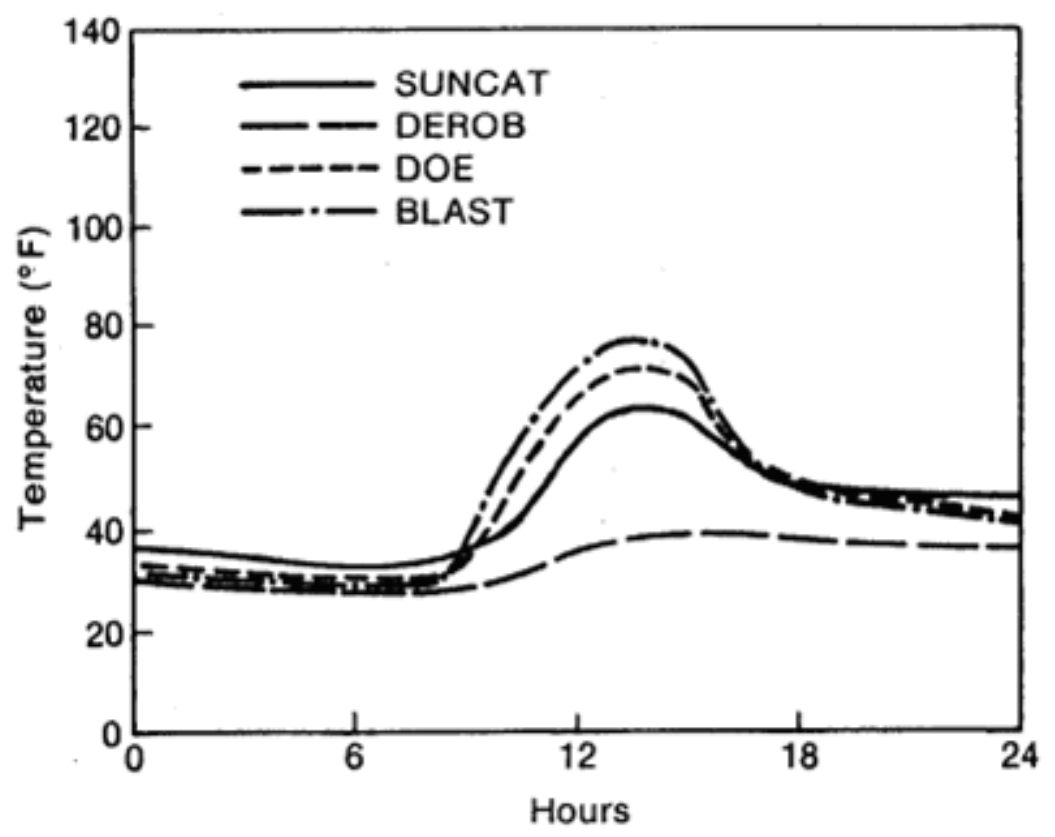

Figure 4-6. January 21 low-mass room air temperature (Madison)

For the low-mass case, the SUNCAT-2.4, DOE-2.1, and BLAST-2MRT curves are similar in shape and amplitude. They demonstrate the quick response and relatively large temperature excursions expected in a highly solar-driven, direct-gain building with neither thermal storage nor night insulation. The DEROB-3 low-mass temperature profile is much flatter, more representative of a high-mass building.

The high-mass profiles show the same trend. The SUNCAT-2.4, DOE-2.1, and BLAST-2MRT curves are similar in shape, amplitude, and phase, while the DEROB-3 curve remains much flatter. In all cases except DEROB-3, the characteristic damping of temperature swings associated with the addition of thermal storage is exhibited. DEROB-3 shows little difference between the high- and low-mass temperature profiles.

\subsubsection{Annual Heating and Cooling Loads}

Annual heating and cooling loads for the high- and low-mass cases are shown in Figures 4-7 and 4-8. SUNCAT-2.4, BLAST-2MRT, and DOE-2.1 agree closely for heating and cooling. These three codes all exhibit substantially reduced loads when mass is added. DEROB-3, on the other hand, displays a relative insensitivity to changes in thermal storage mass. 


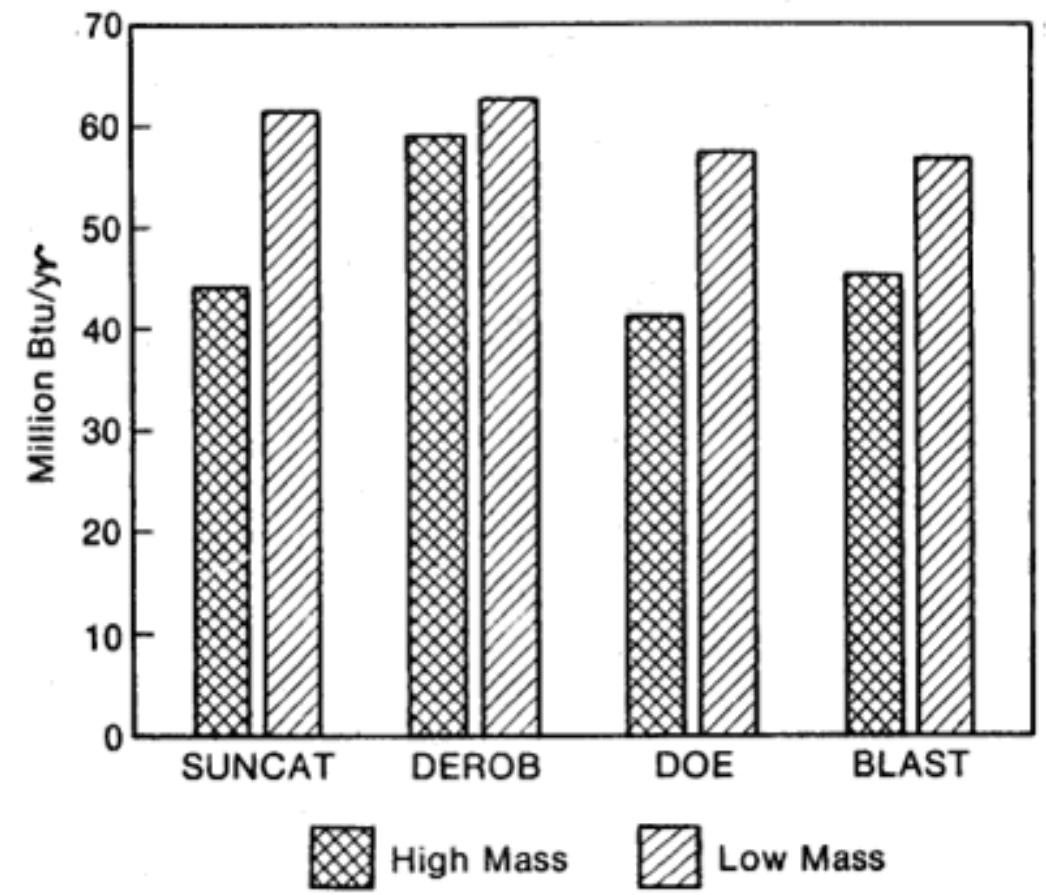

Figure 4-7. Annual heating loads for four codes (Madison)

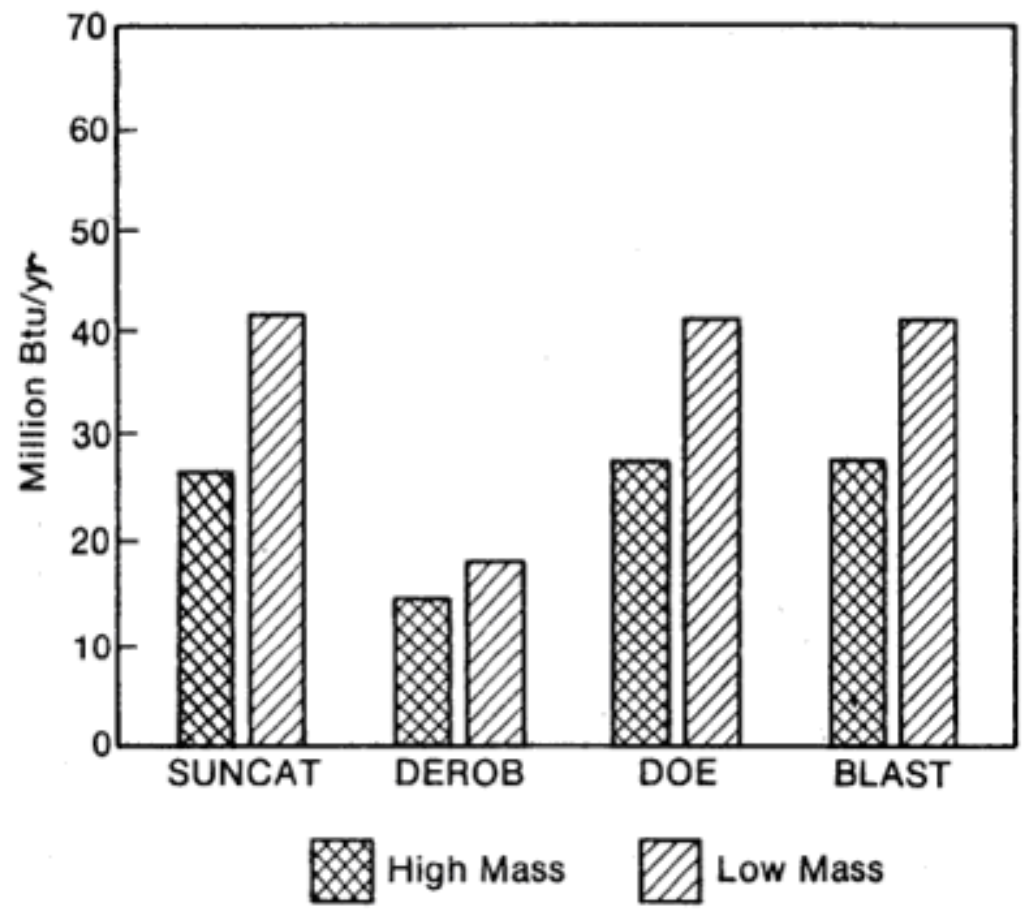

Figure 4-8. Annual cooling loads for four codes (Madison)

\subsubsection{Discussion}

As previously stated and based on the hourly profiles, it appears that DEROB-3 is relatively insensitive to changes in thermal mass. If this hypothesis were true, we would expect to see agreement between DEROB-3 and the other codes for high-mass annual heating and cooling loads. This is true for cooling but not for heating. For the heating case we saw that DEROB-3 
agreed within the range of error shown by the other programs for low mass but not for high mass.

By using the analytical tests for temperature decay, as illustrated in Figures 4-9 and 4-10 (see Section 5.0 for details), we were able to isolate this problem to the solution technique used in the sixth subprogram of DEROB-3, DBROLEN. To avoid a costly matrix inversion, we used an iterative solution technique. The iterations are controlled by the variable LOOM in the RCSOLN subroutine. This variable is fixed at one iteration and is not normally available to the user. The solution uses the last hour's temperature and then iterates once in attempting to achieve convergence. This assumption is valid where surface temperatures are changing slowly. However, where surface temperatures change rapidly, as in a low-mass surface or a high-mass surface receiving direct solar gain, one iteration does not allow surface temperatures to change quickly enough. Thus, surface temperatures are mathematically constrained to small differences over each time step. In other words, radiant or thermal energy causes a small temperature change on surface nodes not because the energy is absorbed by thermal mass, but rather because a first law energy balance is not performed. The gain or loss of energy that would be found from an energy balance is simply unaccounted for.

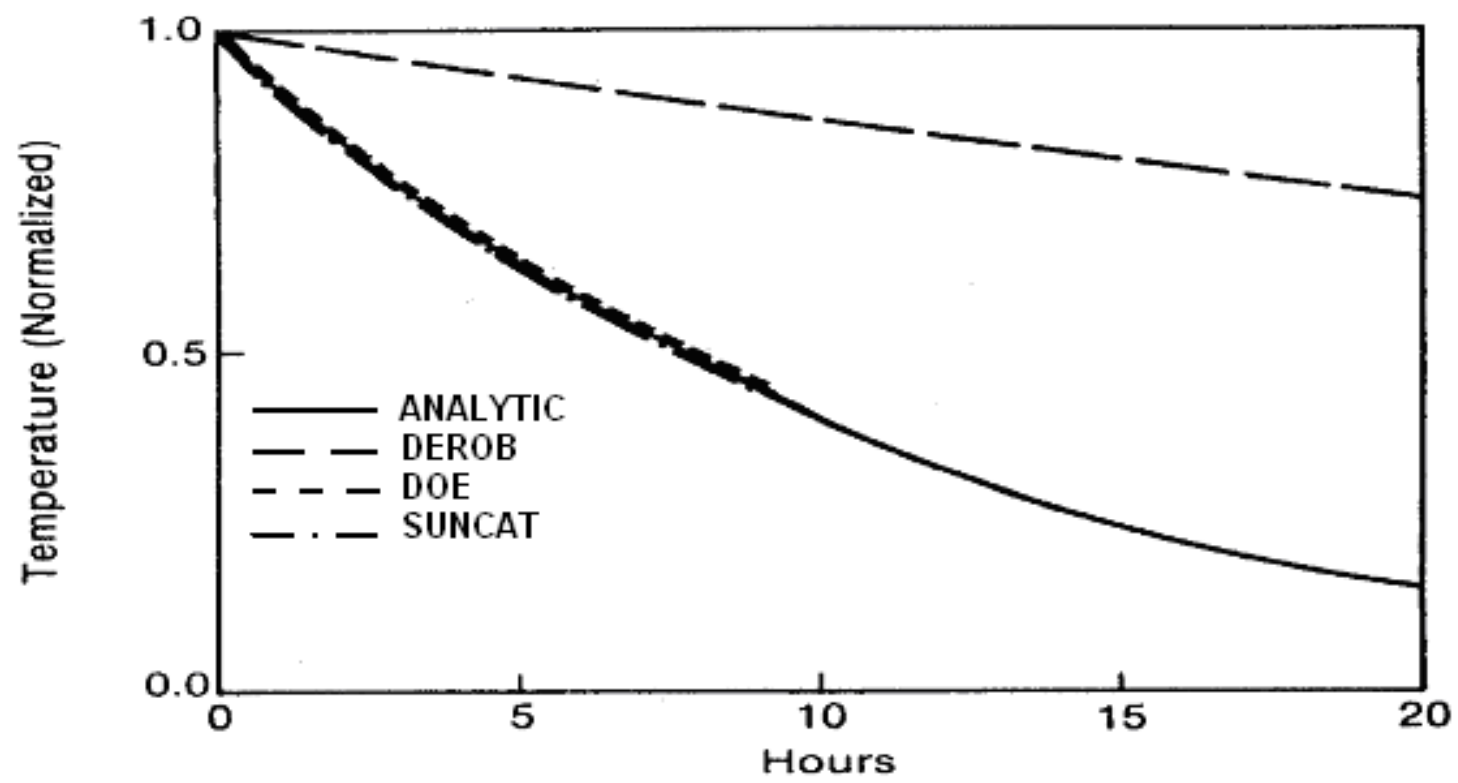

Figure 4-9. Low-mass temperature decay tests for three codes 


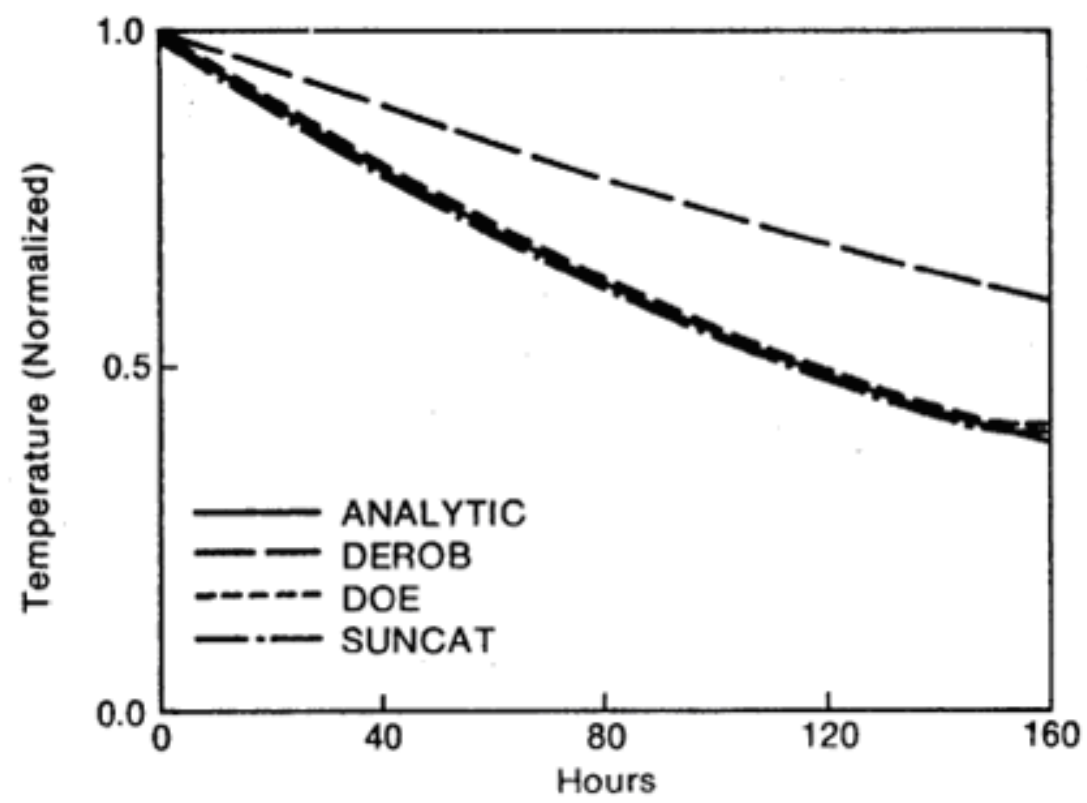

Figure 4-10. High-mass temperature decay tests for three codes

The temperature profiles shown in Figures 4-5 and 4-6 always appear characteristic of a very high-mass building. The long-term energy results (see Figures 4-6 and 4-7), on the other hand, are explained by the fact that surface nodes are slow in changing temperatures. In the high-mass heating case, surface temperatures never rise enough to drive energy into storage, so DEROB-3 shows more heating load. In the low-mass heating case, better agreement was shown because this effect was dominated by losses through the collection area at night. Even though the other programs showed large temperature swings on January 21 and DEROB did not, the combined effect of very low storage and no night insulation caused the auxiliary energy results to appear similar. In the cooling case, this works in the opposite way. That is, DEROB-3 now appears to be in better agreement for the high-mass case and in poor agreement for the low-mass case. This is again caused by the slow temperature response of DEROB-3. The other programs exhibit high cooling loads because temperatures are spiked. In DEROB-3 temperatures do not rise rapidly, so low cooling loads ensue. In the high-mass case the other codes show reduced load because of flywheel effect. DEROB-3 appears to behave similarly because temperatures are mathematically constrained.

\subsubsection{Conclusions}

- In the test cases described, BLAST-2MR.T, SUNCAT-2.4, and DOE-2.1 show substantial agreement for long-term heating and cooling with maximum differences of less than $1 \%$.

- All four codes show differences in hourly temperature response. These may or may not be significant in the range of parameters commonly associated with buildings. Another comparative study is planned to further investigate this question.

- DEROB-3 showed insensitivity to the mass parameter. It diverged from the other three codes for both the hourly temperature profiles and the yearly energy usage. This code also disagreed considerably with the high- and low-mass analytical temperature decay 
tests. The source of these errors is in the subroutine RCSOLN in the subprogram DBROLEN.

- The use of an anisotropic versus an isotropic sky model in the DOE-2.1 radiation processor caused differences of approximately $10 \%$ in predicted annual heating and cooling loads.

- The analytical and comparative techniques used together are a powerful diagnostic tool.

\subsection{Phase II Comparative Study: DOE-2.1, BLAST-3.0, DEROB-4, and SUNCAT-2.4}

This section describes the results from Phase II of the comparative studies, which was structured to answer certain questions that arose out of the Phase I study. The specific questions are as follows:

- The Phase I study showed DOE-2.1, SUNCAT-2.4, and BLAST-2MRT agreeing closely for annual heating and cooling loads (see Figures 4-7 and 4-8); however, some discrepancy was seen in hourly temperature profiles (see Figures 4-5 and 4-6). Was the close agreement in annual loads coincidental, or would disagreement increase as the building description and weather were altered from the original case?

- Phase I revealed a flaw in the DEROB-3 thermal solution technique causing DEROB-3 to be insensitive to variations in thermal mass. Since that time a new version of the code, DEROB-4, has been written. Was the problem corrected in DEROB-4?

- Since the Phase I study, a new version of BLAST, BLAST-3.0, has been written. How would this new version perform?

\subsubsection{Test Building Characteristics}

We changed our building model slightly from Phase I to Phase II. In Phase I, the groundcoupling mechanisms in the codes were crippled by using a very thick layer of insulation in the floor to minimize differences in the results from the different ground-coupling algorithms in the codes. However, this caused a minor input inconsistency. SUNCAT, DOE, and BLAST are capable of modeling pure resistance, whereas DEROB either associates capacitance with an insulating material, or models the surface as if it were adiabatic. This problem was eliminated in Phase II by modeling the floor as if the building were hovering in space leaving all exterior surfaces exposed to ambient air. One other difference between the Phase I and Phase II building models was in the thickness of insulation used. In Phase I, the building overall heat-loss coefficient (UA) was kept at a constant $300 \mathrm{Btu} / \mathrm{h}{ }^{\circ} \mathrm{F}$ for both the high- and the low-mass cases. We varied the thickness of insulation to compensate for the difference in resistance between 0.5 in. gypsum board and 8 in. of concrete. This led to very odd insulation thicknesses. In Phase II, it was easier to standardize the wall, roof, and floor insulation thicknesses and let the UA overall of the building vary slightly between the high- and low-mass cases. Table 4-2 shows the thermal characteristics of the Phase II test building. 
Table 4-2. Phase II Building Thermal Characteristics

\begin{tabular}{|l|l|}
\hline Floor area & $1500 \mathrm{ft}^{2}$ \\
\hline Glazing area & $350 \mathrm{ft}^{2}$ \\
\hline Roof section & $\begin{array}{l}0.5 \text {-in. gypsum board } \\
6 \text {-in. Styrofoam (R24) }\end{array}$ \\
\hline Floor and wall section (high mass) & $\begin{array}{l}\text { 8-in. concrete } \\
\text { 3-in. Styrofoam (R12) }\end{array}$ \\
\hline Floor and wall section (low mass) & $\begin{array}{l}0.5 \text {-in. gypsum board } \\
3 \text {-in. Styrofoam (R12) }\end{array}$ \\
\hline Dead band & $65^{\circ}-75^{\circ} \mathrm{F}$ \\
\hline Infiltration & $0.65 \mathrm{ACH}$ \\
\hline No shading & \\
No night insulation, no ground coupling & \\
Zero external absorptivity, single zone & \\
\hline
\end{tabular}

\subsubsection{Annual Heating and Cooling Loads}

To determine if the disagreement in hourly temperatures predicted by the codes in the first comparative study could lead to significant discrepancies in annual load predictions, the building was simulated using Madison and Albuquerque weather data to test the simulations under two climatic extremes. Madison represents a condition in which conductive losses dominate the performance of the building, whereas building performance in Albuquerque reflects solar gain. Different combinations of mechanisms are stressed by these two locations even though the building remains the same.

Figures 4-11 and 4-12 show the annual heating and cooling consumption of all four codes in Albuquerque and Madison for the high- and low-mass cases.

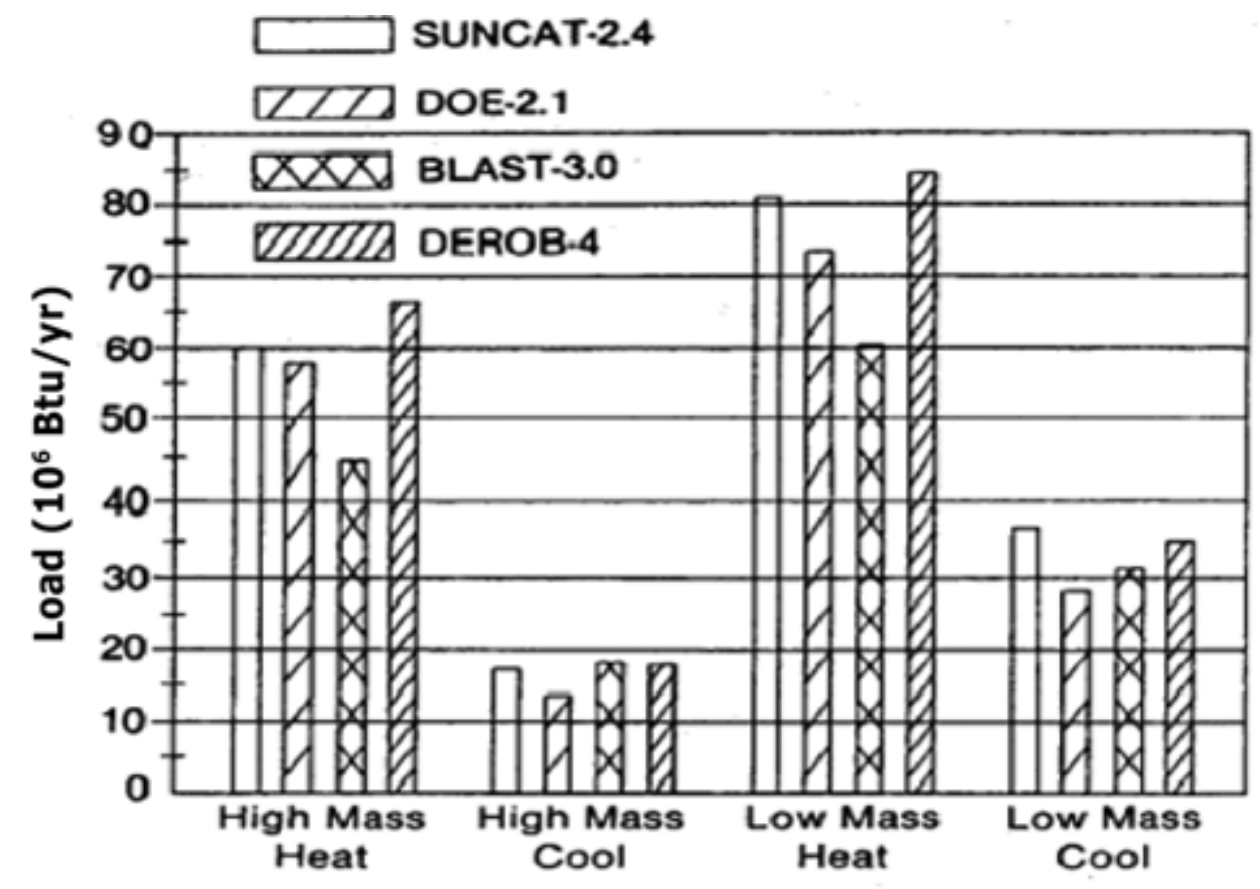

Figure 4-11. Phase II comparative study (Madison) 


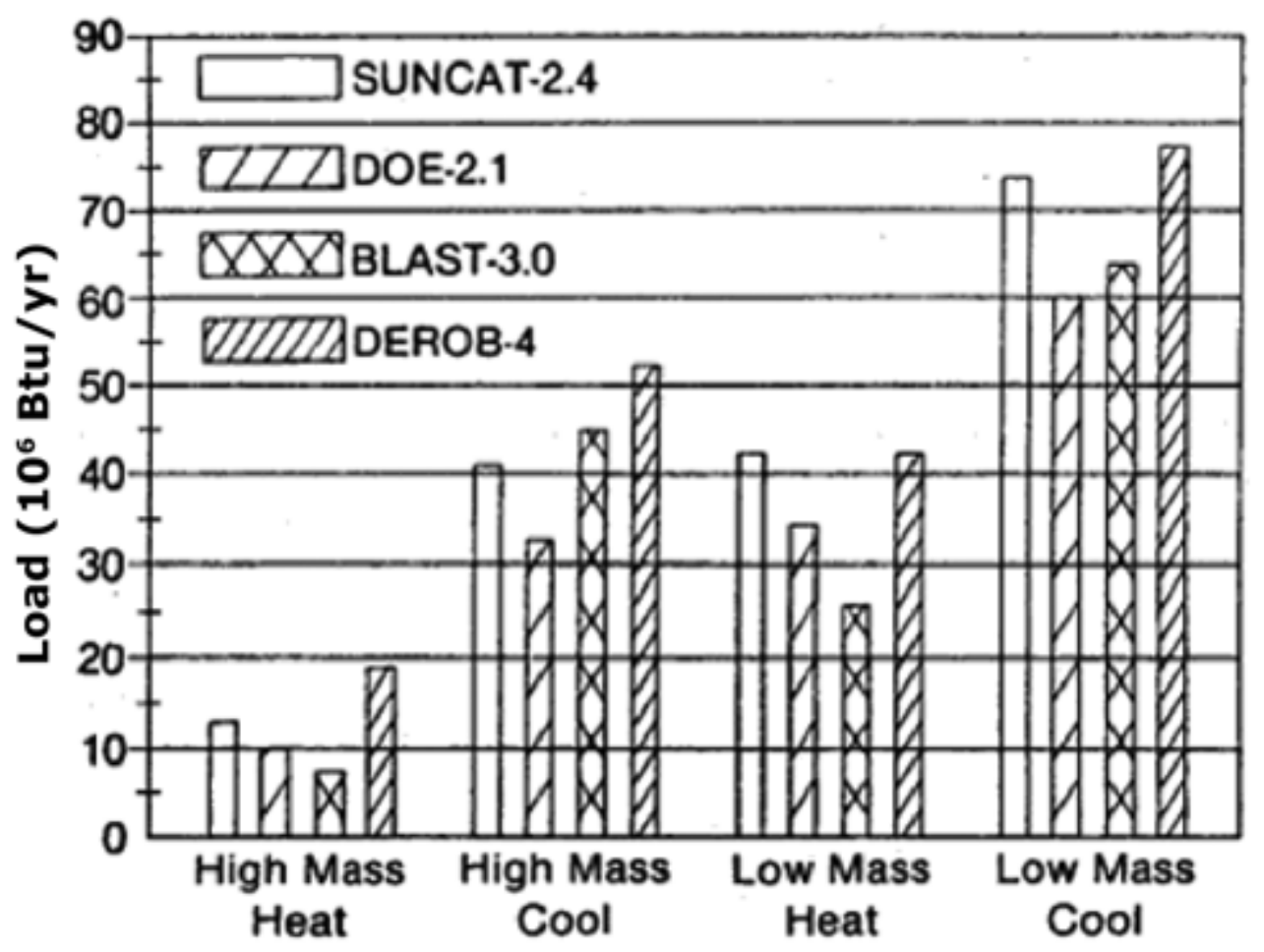

Figure 4-12. Phase II comparative study (Albuquerque)

Although considerable scatter is apparent, certain patterns are discernible. BLAST-3.0 is low in annual heating load in all cases. The positions of the codes are relatively consistent in all the cases; i.e., DEROB always shows the highest heating loads, BLAST the lowest, SUNCAT the second highest, and DOE the second lowest. Cooling is similarly consistent. Additionally, all codes show the same tendencies in the direction of response to parameter changes, even though the magnitudes of these responses are different.

Figures 4-13 and 4-14 show the annual energy consumption for SUNCAT and DOE-2.1 for the Phase I and Phase I-I building model. The Phase I Madison results for high mass were very close for both heating and cooling, the greatest difference being $0.8 \%$ for cooling. The Phase II Madison results show about a 3\% difference in heating and a $23 \%$ difference in cooling. The Albuquerque case shows the greatest divergence, with a $23 \%$ difference in heating and a $20 \%$ difference in cooling. The low-mass results show a similar pattern of divergence.

Figure 4-15 shows the responsiveness to changes in thermal mass for DEROB-3 and DEROB-4. In Phase I, DEROB-3 proved insensitive to changes in thermal mass. This problem appears to be corrected in DEROB-4. This conclusion is supported by the results from the analytical verification procedure where the temperature curves for thermal mass charge and decay in DEROB-4 match the analytical solutions (Section 5.0). 


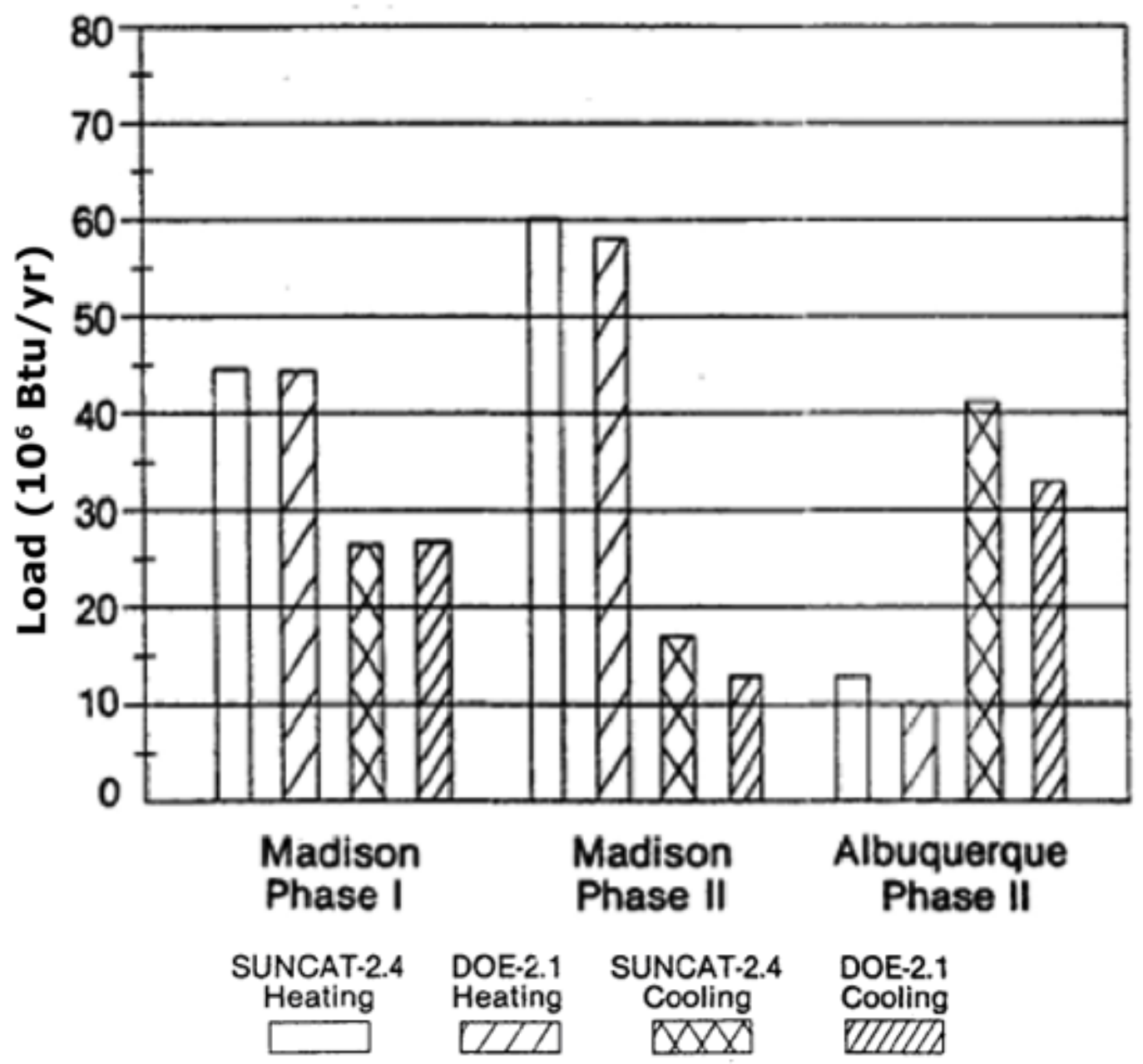

Figure 4-13. Annual energy consumption-high-mass results 


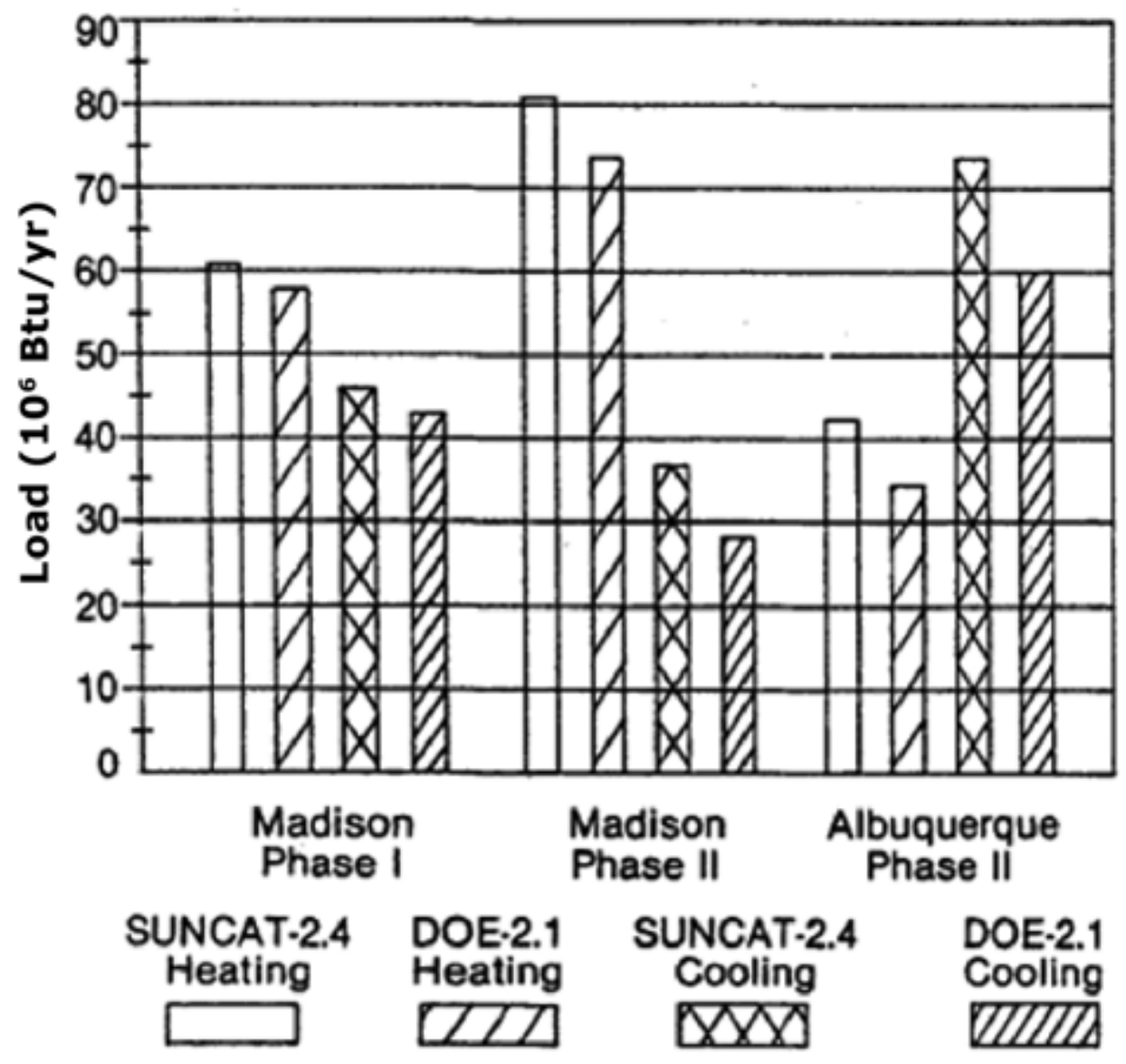

Figure 4-14. Annual energy consumption-low-mass results 


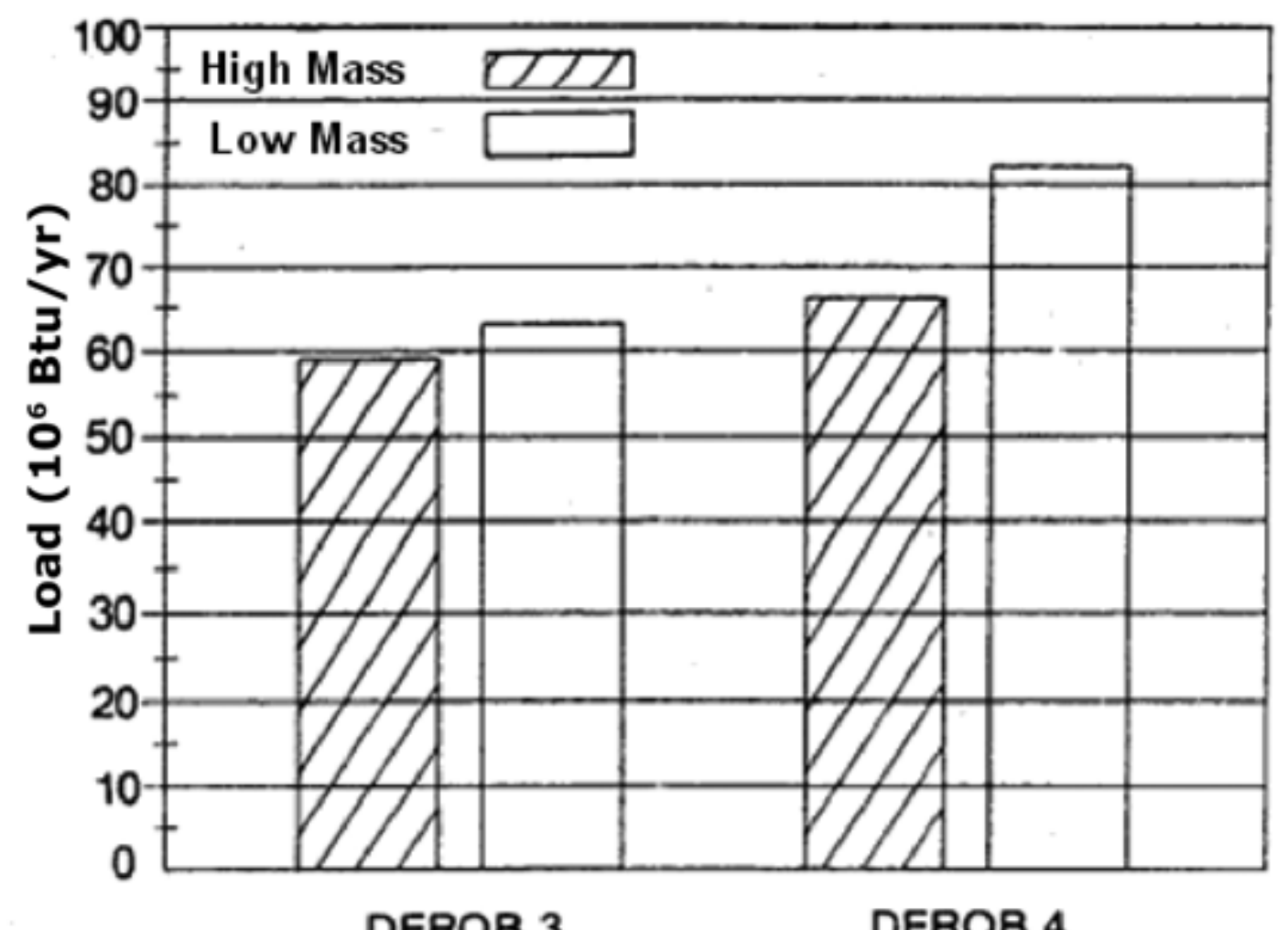

Figure 4-15. Sensitivity to changes in thermal mass

\subsubsection{Discussion}

The results illustrated in Figures 4-11 and 4-12 suggest that the close agreement obtained between DOE-2.1, BLAST-2MRT, and SUNCAT-2.4 in the Phase I comparative study may have been a coincidence of the particular mix of parameters chosen for that case. Even a relatively small perturbation of the parameter mix as represented in Figures 4-13 and 4-14 by the change from the Phase I Madison case to the Phase II Madison case results in significant disagreement between SUNCAT-2.4 and DOE-2.1. DEROB-4 and BLAST-3.0 are rewritten versions of the codes used in the Phase I study, so the increased disagreement in Phase II could be attributed to the differences in the rewritten versions. However, the SUNCAT-2.4 and DOE2.1 programs were used for both studies. Therefore, the increased disagreement between these two codes can only be attributed to the differences in the Phase I and Phase II building models and the Madison and Albuquerque weather data (Figures 4-13 and 4-14). This is confirmed in the Albuquerque case where we observe the disagreement between DOE-2.1 and SUNCAT-2.4 increasing as the divergence from the Phase I Madison case becomes more extreme.

The magnitude of disagreement among all four codes is surprising in light of the results obtained in the analytical verification study, where these programs all agreed quite closely with a number of analytical solutions. These analytical solutions were chosen to test the most important individual and combined heat transfer mechanisms in the codes. 
There are three possible explanations for this apparent conflict: (1) input errors to the codes for the comparative study; (2) important heat transfer mechanisms neglected; and (3) different treatment of coupled mechanisms by the three codes.

We cannot determine at this stage which is the correct explanation; however, the third explanation appears to be the most probable. The first explanation is unlikely because of the consistent pattern in the results. The second is doubtful because of the care taken in defining a "common denominator building"; i.e., a very simple building model with characteristics well within the computational capabilities of all simulations in the study. Additionally, great care was taken to include those mechanisms most important to building thermal performance in the analytical test-set.

If the third possibility is correct, caution should be exercised in interpreting validation studies that only display temperatures, since even apparently small differences in temperature can under certain conditions lead to relatively large differences in predicted loads. For example, if one code consistently predicts temperature excursions just outside the heating and cooling dead band, and another code predicts temperature swings just within the dead band, the cumulative energy predictions can be quite far apart even though the temperatures are close.

\subsubsection{BLAST-3.0}

BLAST-3.0 represents a considerable rewrite from BLAST-2MRT. In the Phase II study, BLAST-3.0 is consistently low in annual heating load prediction. However, in the absence of a truth model it is impossible to say whether BLAST-3.0 or one of the other programs is correct. BLAST-3.0 performed adequately on all of the major analytical tests, as did all of the other programs.

\subsubsection{Conclusions}

- Significant disagreement exists in predicting annual heating and cooling loads among all the codes even when a very simple building model is used.

- The analytical verification study showed substantial agreement between the treatment of major heat transfer mechanisms in the codes and the analytical solutions. This suggests that the discrepancies between the codes are occurring as a result of the coupling mechanisms, rather than as a result of the mishandling of any mechanisms in individual components.

- DEROB-4 has successfully corrected the mass insensitivity problem in DEROB-3 uncovered in the Phase I Comparative Study.

- Caution should be exercised in using these simulations for economic analysis or the generation of design tools because of the magnitude of discrepancy observed in predicting annual heating and cooling loads. These programs show trends and tendencies; however, their sensitivities to parametric changes differ somewhat.

- Further investigation is needed to determine the causes of these differences, and which program, if any, is most accurate in predicting the thermal behavior of buildings. We are currently collecting high-quality empirical data from our own two-zone test cell and a four-zone full-scale residence. Additionally, we have defined our data needs for the test 
facilities at CSU in Fort Collins and at the NBS (see Section 6.0). We will present the results of these empirical validation exercises in future publications.

\subsection{References}

1. Judkoff, R. et a1. 1980. "A Comparative Study of Four Passive Building Simulations: DOE-2.1, BLAST, SUNCAT-2.4, DEROB-3," Proceedings of the Fifth National Passive Solar Conference, pp. 126-130, Oct. Also SERI/TP-721-837, Golden, CO: Solar Energy Research Institute.

2. Judkoff, R. et al. 1981. "A Comparative Study of Four Building Energy Simulations: Phase II: DOE-2.1, BLAST-3. 0, SUNCAT-2.4, and DEROB-4," Proceedings of the Sixth National Passive Solar Conference, July. Also SERI/TP-721-1326, Golden, CO: Solar Energy Research Institute.

3. York, D.A. and Tucker, E.F., eds. 1980. DOE-2 Reference Manual (Version 2.1).

4. York, D.A. and Tucker, E.F., eds. 1980. DOE-2 Reference Manual (Version 2.1), Group WX-4, Program Support J Los Alamos, NM: Los Alamos Scientific Laboratory.

5. Hittle, D.C. 1977. The Building Loads Analysis and System Thermodynamics Program,

6. Volume I: User's Manual. Champaign, IL: Construction Engineering Research Laboratory.

7. Hittle, D.C. 1979. The Building Loads Analysis Thermodynamics (BLAST) Program:

8. Users Manual. Champaign, IL: Construction Engineering Research Laboratory.

9. Arumi-Noe, F. and Wysocki, M. 1979. DEROB III, The DEROB System, Vol. 1-8.

10. Palmiter, L. 1980. SUNCAT Version 2.4: User Notes. Butte, MT: National Center for Appropriate Technology.

11. Wortman, D. et a1. 1981. "The Implementation of an Analytical Verification Technique on Three Building Energy Analysis Codes: SUNCAT 2.4 J DOE-2.1 and DEROB III," Proceedings of the ASME/SSEA Conference. 


\subsection{Analytical Verification}

\subsection{Philosophy}

Analytical verification is a technique to test selected heat transfer mechanisms common to many building simulation codes [1]. The technique compares the output from a code's numerical solution to a unique solution of a well-defined heat transfer problem. Conduction and solar charging of mass are the main mechanisms studied. These mechanisms were chosen both for their importance to building energy flows and the ease with which the test could be implemented. Analytical verification can detect major problems in a code, although it is most useful in determining selected numerical solution or modeling assumptions and in affirming the user's understanding of a code.

Analytical verification can be related to the validation process, as shown in Figure 3-3. The building energy problem to be solved consists of a simple building shell exposed to weather data whose time dependence is some analytical expression. The solution to this type of problem requires a set of assumptions. For example, one can assume that wall conduction is one dimensional and that zone air is isothermal. Also, assumptions are made that determine values for surface absorptivities and film coefficients. Once the assumptions are made and the problem is defined precisely, the problem can be solved in two ways: (1) by using a building energy analysis simulation code, or (2) by finding the unique solution to the analytical expression of the problem. Analytical verification can thus be defined as a series of comparisons between the outputs from a code and the analytical solutions given a set of common assumptions.

Use of the analytical solution depends on the problem to be solved. In some cases, the analytical solution is considered as the truth model against which the output from the simulation is evaluated. These cases include wall conduction and solar charging of thermal mass. When the simulation results differ from the analytical solution, possible sources of disagreement are:

- The code is in error.

- The problem was incorrectly input into the code or the code output has been interpreted incorrectly.

- The assumptions have been incorrectly applied to the code or assumptions inherent in the code have not been accounted for.

The analytical solutions can be used to determine other information about the codes. The solutions provide information that helps to identify selected modeling parameters in a code such as volumetric heat capacity of air. The correctness of these values is not determined by the solution, but rather they must be evaluated by the use of engineering judgment.

The strengths and weaknesses of the analytical verification approach are summarized in Table 51. A major advantage to the technique is that all parameters are exactly defined for the problem. These parameters can be varied essentially at will, since the analytical solutions allow any value that does not violate the assumptions associated with the problem. Thus, a code's dependence on a given parameter can be easily seen and understood. Also, since one has complete control of both the building model and the weather data, the problems can be made simple enough that only 
selected mechanisms are present. For example, solar radiation effects can be eliminated by setting appropriate solar absorptivities and solar radiation values to zero. This allows the study of the wall conduction mechanism in isolation from solar effects.

Table 5-1. Analytical Validation Assessment

\begin{tabular}{|l|l|}
\hline \multicolumn{1}{|c|}{ Strengths } & \multicolumn{1}{c|}{ Weaknesses } \\
\hline All problem parameters are "exactly known" & $\begin{array}{l}\text { Makes no test of correctness of the common } \\
\text { modeling assumptions }\end{array}$ \\
\hline $\begin{array}{l}\text { Parameters can be varied at will, solution is } \\
\text { general }\end{array}$ & $\begin{array}{l}\text { Difficult to ensure same modeling assumptions } \\
\text { between code and solution }\end{array}$ \\
\hline Allows isolation of selected mechanisms & $\begin{array}{l}\text { Only simple problems, incomplete set of } \\
\text { mechanisms }\end{array}$ \\
\hline
\end{tabular}

Analytical verification also has certain weaknesses that must be understood. First, both the code and the solution are based on assumptions whose correctness must be determined. These assumptions should ultimately be compared against empirical data. Second, since the code and the analytical solution must follow the same assumptions, the analytical verification method can be difficult to implement because the codes can have many implicit assumptions concerning the behavior of heat-transfer mechanisms. Thus, once a problem is chosen, it is often necessary to modify the simulation source code to ensure that it conforms to the assumptions in the problem. For example, the solutions presented in this section assume constant film coefficients, and several codes incorporate more complex models for those coefficients that must be modified. The modification must be done very carefully, and code authors must be consulted if any doubts exist.

The third drawback of analytical verification involves the selection of the weather data. The step function weather parameter changes in the analytical solutions presented here can provide information about the performance of heat transfer mechanisms. However, this type of driving function is not closely related to the real weather patterns that a building would experience. Codes with algorithms that use normal weather data could have problems with the synthetic weather data used in these tests. Thus, if a code gives correct results for these tests, one cannot conclude that the code will give accurate results for real buildings using real weather data. Normal weather patterns could be simulated by other analytical forms (sine wave for ambient temperature and a rectified sine wave for solar radiation) and further analytical solutions could be developed. However, work on these analytical solutions is not included in this report.

Finally, analytical verification tests only selected heat transfer mechanisms that are not inclusive. The bulk of these tests check mechanisms operative in individual components with no testing of component coupling mechanisms. Even though mechanisms in individual components appear correct when tested with this technique, interactions between these individual components can have a large effect on results. These interactions are not tested by this technique. Note also that no attempt is made to test equipment, loads, or schedules that are so important to internal loaddominated buildings. Thus, it cannot be assumed that a code will accurately predict a real building's energy performance on the basis of these tests.

This report contains the information needed to test a building energy analysis simulation code by analytical verification and presents a detailed description of how to implement the tests. The rest 
of this section is divided into a discussion of the theoretical basis for the tests and the weather data. A step-by-step procedure for running the tests is presented in Appendix A. Also included is a presentation of the results of running the tests on SUNCAT-2.4, DOE-2.1, DEROB-3, DEROB-4, and BLAST-3.0 and the modifications needed to implement the tests on these codes.

\subsection{Theoretical Background}

The theoretical basis of the analytical verification tests consists of analytical solutions to onedimensional heat transfer problems. These problems were chosen because they are solvable analytically and can be simulated using a variety of building energy analysis simulation codes. The first analytical solution is for a slab of thickness L convectively coupled to the ambient temperature on one side and adiabatic on the other. All heat transfer properties, including the convective coupling coefficients, are held constant. We assumed that all heat transfer is one dimensional. The driving function for the solution is a step function in the ambient temperature, although other functional forms could also be chosen. The problem and its solution are shown in Figure 5-1.

Figure 5-1 presents an infinite series solution for any combination of the thermal parameters. However, a solution to an analogous electrical circuit can, under the proper conditions, be essentially equivalent to the exact analytical solution (see Figure 5-2). This equivalence depends on the value of the Biot number of the mass in the wall. The Biot number is:

$$
\mathrm{Bi}=\mathrm{U}_{\mathrm{o}} \mathrm{L} / \kappa
$$

Where,

$\mathrm{U}_{\mathrm{o}}=$ the total conductance between the wall midpoint and the ambient air $\left(\mathrm{W} / \mathrm{m}^{2}{ }^{\circ} \mathrm{C}\right)$

$\mathrm{L}=$ the thickness of the wall $(\mathrm{m})$

$\kappa=$ the thermal conductivity of the wall material $\left(\mathrm{W} / \mathrm{m}^{\circ} \mathrm{C}\right)$.

Equivalence can be assumed if the Biot number is less than 0.1, which means that the mass wall is essentially isothermal and film resistance $1 / \mathrm{U}_{\mathrm{o}}$ dominates the problem. Now consider three cases, each with a different thermal mass, but with the appropriate film coefficients and insulation levels, as well as the same overall thermal conductance (see Appendix A for more details on the wall constructions).

For the low-mass case, 0.013-m gypsum board:

$$
\mathrm{B}_{\mathrm{i}}=\left(0.44 \mathrm{~W} / \mathrm{m}^{2}{ }^{\circ} \mathrm{C}\right)(0.013 \mathrm{~m}) /\left(0.43 \mathrm{~W} / \mathrm{m}^{\circ} \mathrm{C}\right)=0.01<0.1 .
$$

For the mid-mass case, $0.1-\mathrm{m}$ concrete:

$$
\mathrm{B}_{\mathrm{i}}=\left(0.45 \mathrm{~W} / \mathrm{m}^{2}{ }^{\circ} \mathrm{C}\right)(0.10 \mathrm{~m}) /\left(0.93 \mathrm{~W} / \mathrm{m}^{\circ} \mathrm{C}\right)-0.05<0.1 .
$$

For the high-mass case, $0.178-\mathrm{m}$ concrete:

$$
\mathrm{B}_{\mathrm{i}}=\left(0.47 \mathrm{~W} / \mathrm{m}^{2}{ }^{\circ} \mathrm{C}\right)(0.178 \mathrm{~m}) /\left(0.93 \mathrm{~W} / \mathrm{m}^{\circ} \mathrm{C}\right)-0.09<0.1 \text {. }
$$



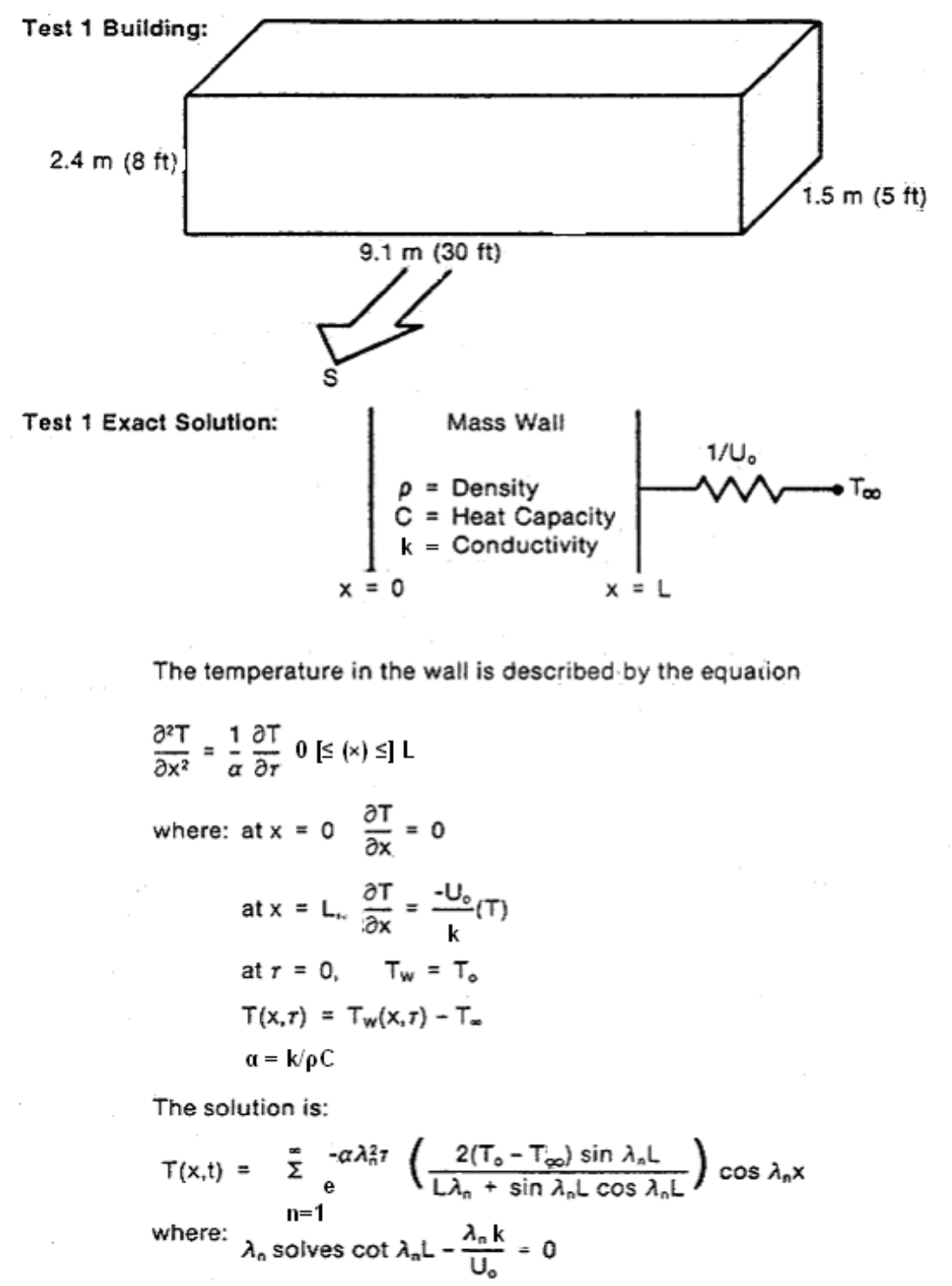

Figure 5-1. Test 1 building and exact solution

Thus, the Biot number criterion is satisfied for each case, and the simpler electrical analogue solution can be used. This is further supported by a comparison between the analytical solution for the temperature of the internal surface of the wall and the electric analogue solution for a high-mass case, shown in Figure 5-3. Note that in the first several hour time steps, there are differences between the infinite series and electrical analogue solutions. These differences arise because in the series solution it takes some time for the effects of the temperature step function to propagate through the entire mass, while in the analogue solution, the entire mass is affected immediately. Thus, these analytical solutions can be used in two ways. The output from the codes can be compared directly with the series solution, or the output with the transients of the first several time steps removed can be normalized and compared to the analogue solution. The second option requires that we ignore enough time steps after the time of the initial step function, so the output from the codes exhibits the exponential form of the analogue solution. We chose the second option for our testing procedure, since it is the easier of the two types of analytical 
solution to implement for a series of wall and material properties. We also present our results from the exact series solutions. These results can also be compared to the output of the simulations.

\section{RC Analogue Circult}

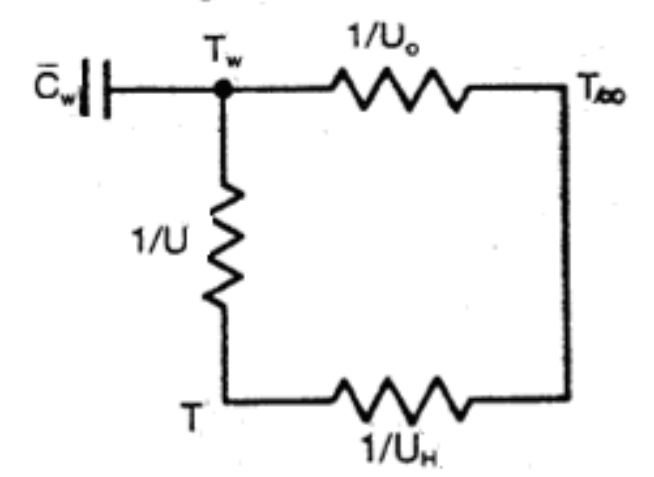

$$
\begin{aligned}
& T=T_{e}(1-e-\tau / \varepsilon)+T_{w_{o}} e+(\tau / \varepsilon) \\
& \text { where: } \varepsilon=\frac{C_{w}}{\left[U_{i} U_{H} /\left(U_{i}+U_{H}\right)\right]+U_{o}}
\end{aligned}
$$

$\boldsymbol{C}_{\boldsymbol{w}}=$ Thermal mass of the wall

$T_{w}=$ Temperature of the wall

$T_{\infty}=$ Ambient temperature

$T_{i}=$ Room air temperature

$U_{o}=$ Conductance between the mid-point of the wall and the outside air

$U_{i}=$ Conductance between the mid-point of the wall and the room air

$U_{H}=$ Conductance between the interior air and the outside air

$$
\begin{aligned}
& \text { If } U_{H}=0.0, \quad \text { Time } \\
& T_{w}-T_{\infty}\left[1-\exp \left(U_{o} / C_{w} / \varepsilon\right) \tau\right]+T_{w o} \exp \left(U_{o} / C W\right) \tau
\end{aligned}
$$

Figure 5-2. Test 1 analogue solution

The second analytical solution is for a slab of thickness L insulated on both edges but receiving a constant radiant flux at one edge. All heat transfer properties are held constant, and heat transfer is assumed to be one dimensional. The problem and its solution [2] are shown in Figure 5-4. The solution in Figure 5-4 has three functionally different components. The first term, $\tau / \rho C L$, is linear with time. The second term, $\mathrm{L} / \mathrm{K}\left[\left(3 \mathrm{x}^{2}-\mathrm{L}^{2}\right) / 6 \mathrm{~L}^{2}\right]$, is a constant for any given $\mathrm{x}$ and is independent of time. The third term, the summation and its factors, defines a transient, the limit of which goes to zero as $\tau$ gets large. Thus, this function takes the following form: 


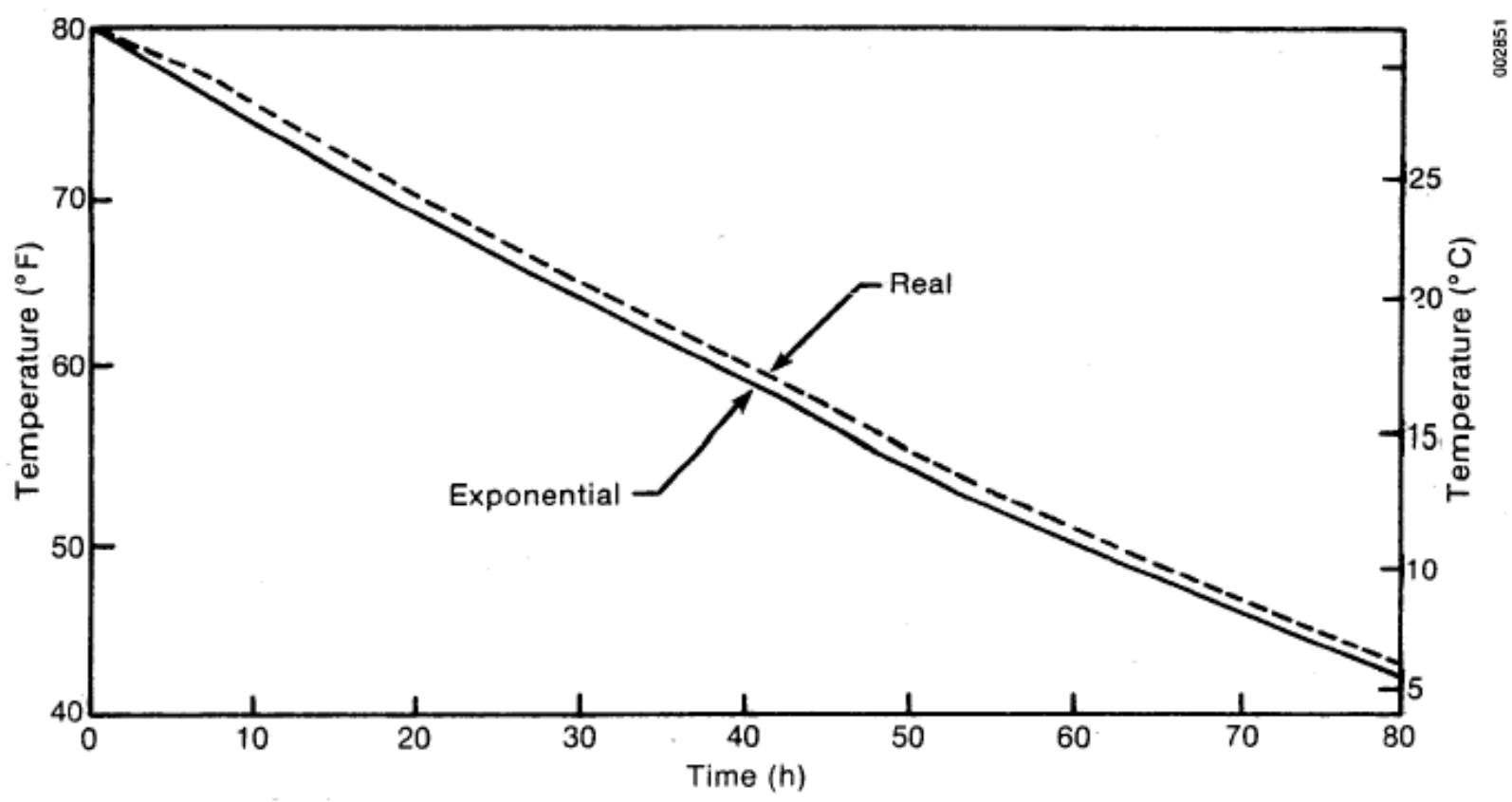

Figure 5-3. Real versus exponential decay for the high-mass case

An initial phase with $\tau$ small where the summation term is always nonzero. This term is subtracted from the linear point-slope form defined by the first two terms. This corresponds to the time $=1$ or 2 hour lines of Figure 5-5 and the nonlinear portion of Figure 5-6. During this time, the radiant flux is being effectively absorbed by only a small portion of the mass wall, and the temperature of this portion of the thermal mass rises quickly.

A final phase with $\tau$ larger where the transient term diminishes. The function closely approaches the linear point-slope form defined by the first two terms. Once this linear phase is reached, the temperature at any distance $\mathrm{x}$ into the wall rises linearly with time and proportionally to the total radiant flux absorbed by the wall divided by the total thermal mass of the wall. This phase is the linear portion of Figure 5-6 and the time $=10,20$, or 100 hour lines of Figure 5-5.

We cannot produce a simpler solution to this problem as we did for the first problem. The analogue circuit solution to the first problem was based on an essentially isothermal condition existing through the thickness of the mass wall. As can be seen in Figure 5-5, the wall always remains distinctly nonisothermal.

\subsection{Weather Data}

Special synthetic weather data files must be developed to perform the analytical verification tests. These files provide both the driving function and selected boundary and initial conditions for the test runs. The weather variables do not correspond to any real weather patterns but are held constant for periods of a month or more. Two types of weather data input files are used. The first, Type A, is used with those tests that require a step function in ambient temperature as the driving function. The Type B weather is used with the tests driven by step functions in diffuse solar radiation. 

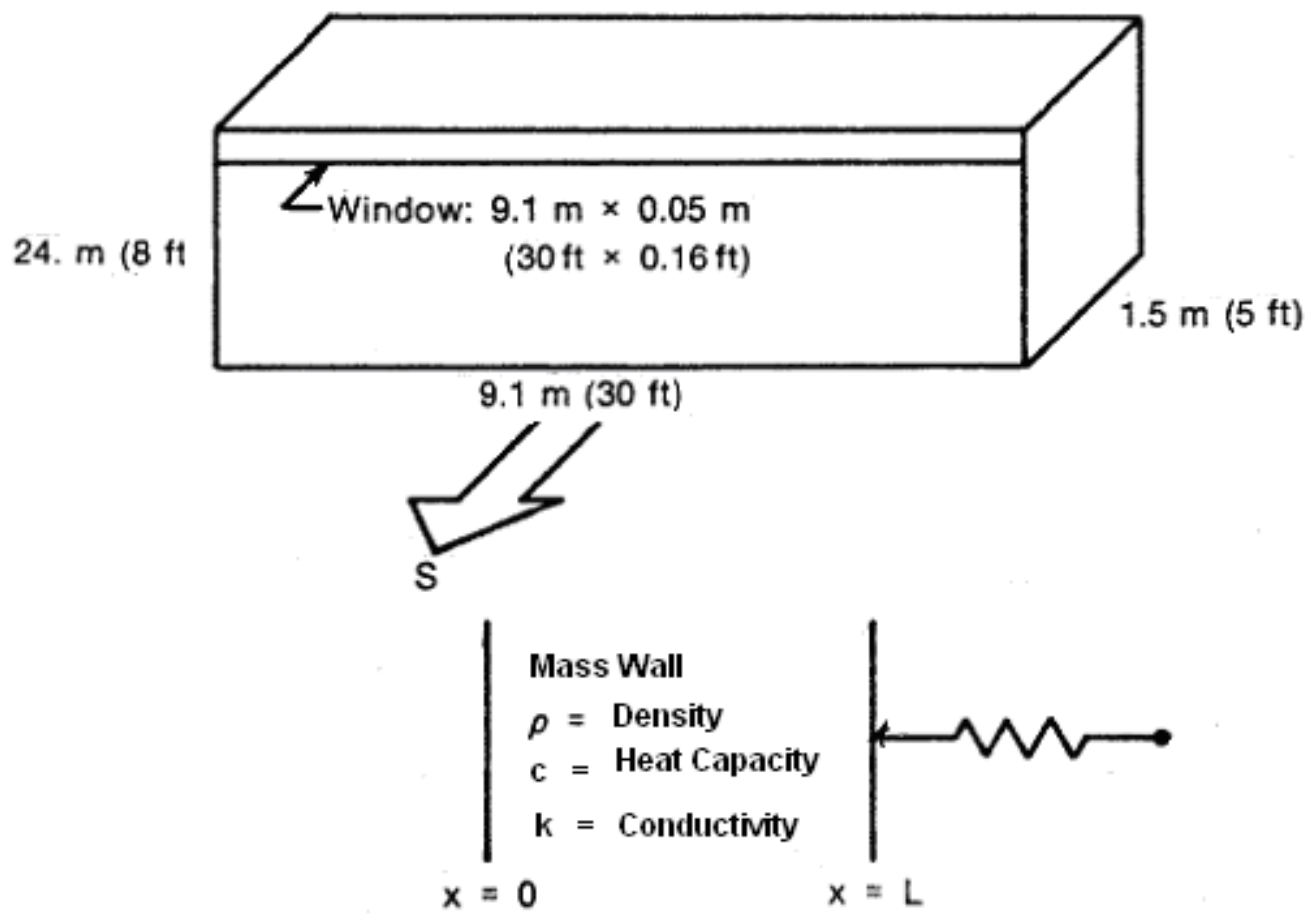

The temperature in the wall is described by the equation

$$
\frac{\partial^{2} T}{\partial x^{2}}=\frac{1}{\partial} \frac{\partial T}{\partial t}, \text { for } 0 \leq \mathrm{x} \leq \mathrm{L}
$$

where: $\alpha=$ wall diffusivity $=\frac{k}{\rho c}$

with boundary conditions:

$$
\begin{gathered}
\mathrm{x}=0: \frac{\partial T}{\partial x}=0 \\
\mathrm{x}=\mathrm{L}: \frac{\partial T}{\partial x}=-\frac{\dot{Q}}{k} \\
\tau=0: \quad \mathrm{T}(\mathrm{x}, 0)=\mathrm{T}_{0}, 0 \leq \mathrm{x} \leq \mathrm{L} \\
\tau>0: \dot{Q}>0
\end{gathered}
$$

The solution is:

$$
\mathrm{T}(\mathrm{x}, \mathrm{t})=\frac{\dot{Q} t}{\rho c L}+\frac{\dot{Q} L}{k}\left[\frac{3 x^{2}-L^{2}}{6 L^{2}}\right]-\frac{2}{\pi^{2}} \sum_{n=1}^{\infty} \frac{(-1)^{n}}{n^{2}} \exp \left(-\alpha \mathrm{n}^{2} \pi^{2} \mathrm{t} / \mathrm{L}^{2}\right) \cos \frac{n \pi x}{L}
$$

Figure 5-4. Radiation step function exact solution and model 
(ft)

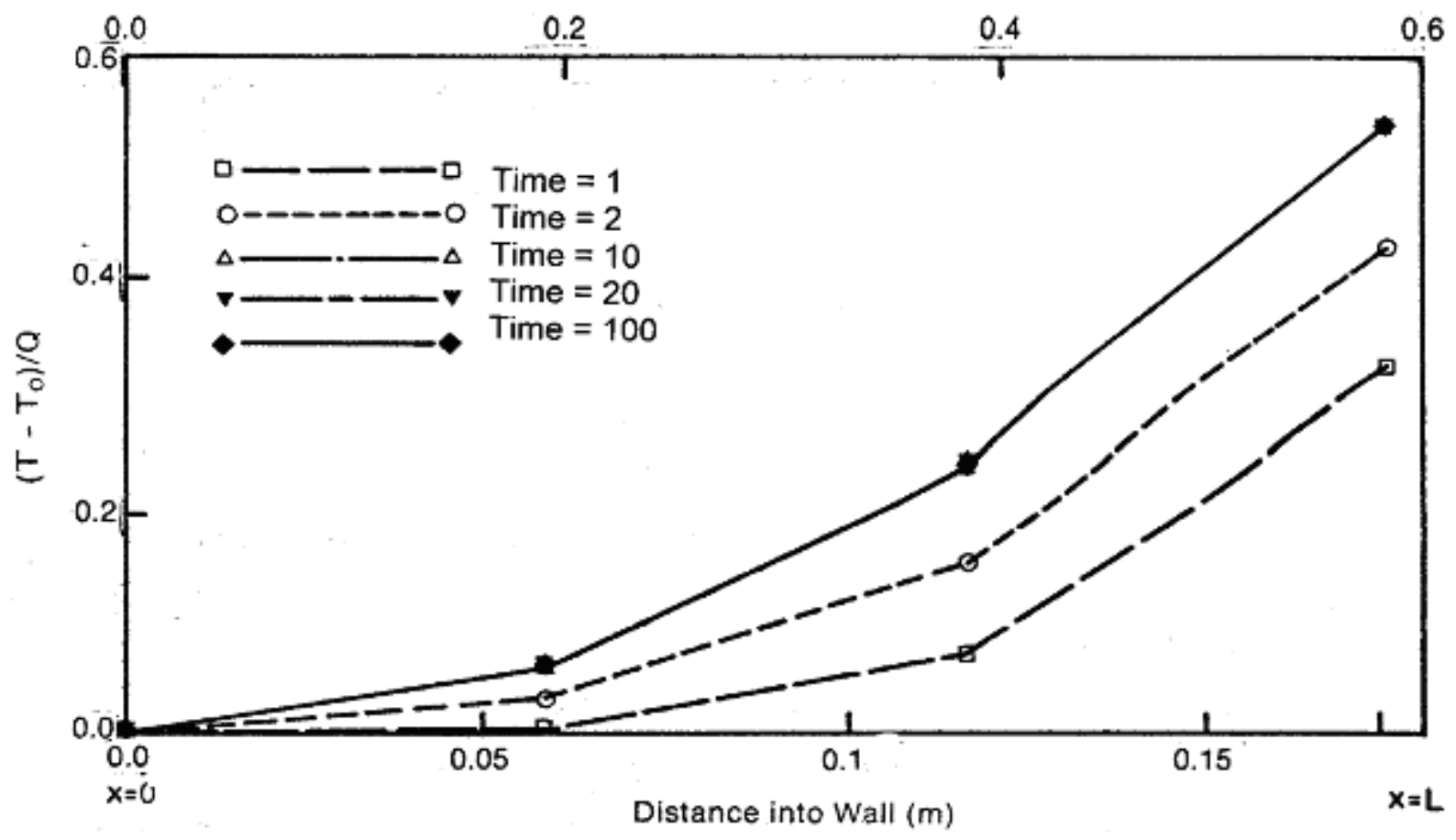

Figure 5-5. Solar charging: propagation of temperature increase through the thickness of a mass wall caused by a constant radiant flux and the wall surface, $X=L$, where $L=$ thickness of wall

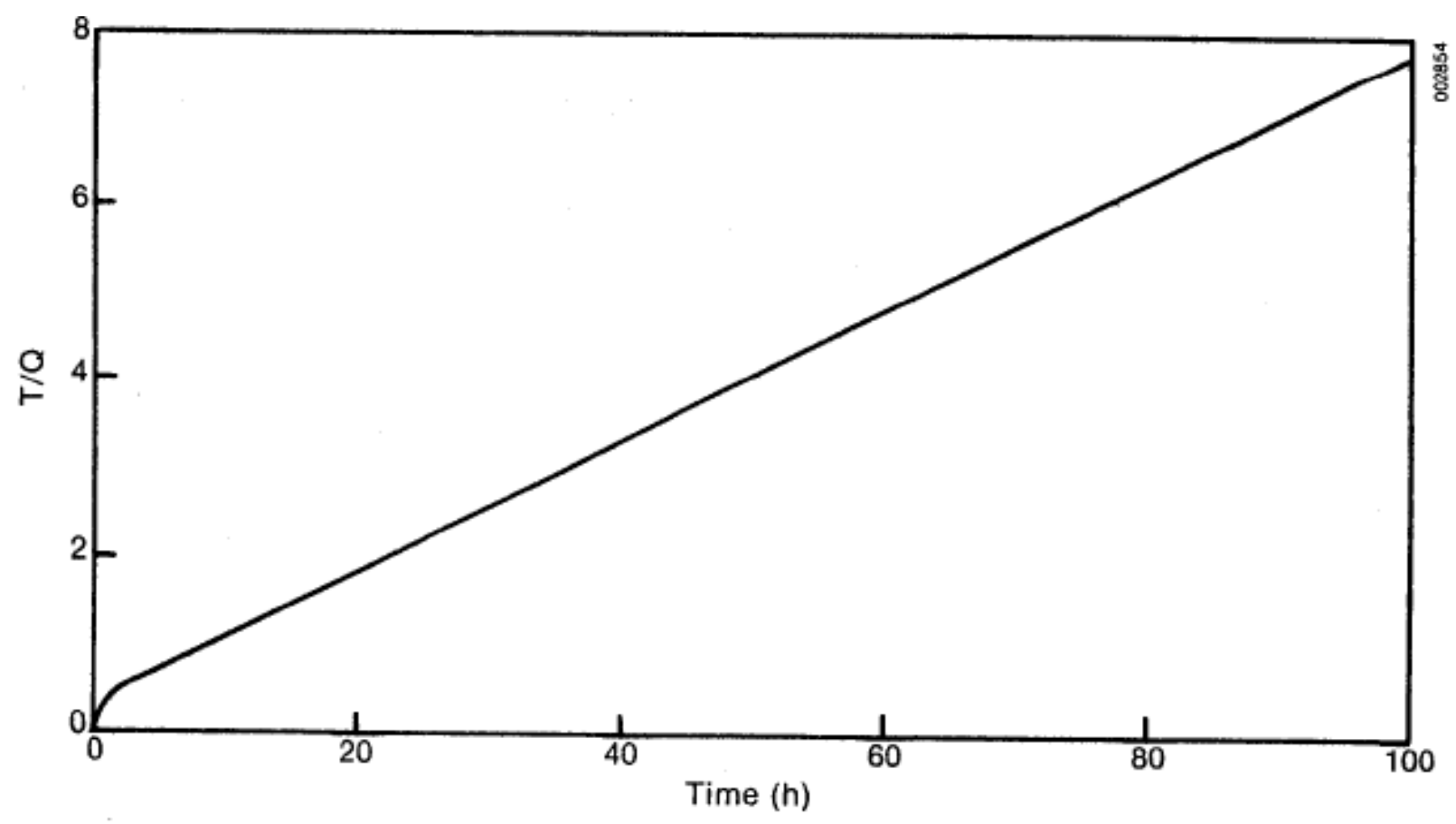

Figure 5-6. Normalized high-mass solar-driven temperature rise versus time

In Sections 5.3.1 and 5.3.2 we describe the values of the variable in the two weather types. These values were chosen because either the variable is the driving function for a test, it establishes 
boundary or initial conditions, or it eliminates the effects of selected mechanisms found in certain codes.

\subsubsection{Type A Weather}

Type A weather is used with tests that require no solar radiation and step functions in temperature as the driving function. The weather variable values used in Type A weather are described in Tables 5-2 and 5-3 and Figure 5-7. Not all of these variables will be used as the input for any one code. In particular, wet-bulb temperature, humidity ratio, and dew point describe the water content of air and are not used together in any one code.

Table 5-2. Type A Weather Data

\begin{tabular}{|l|l|l|}
\hline \multicolumn{1}{|c|}{ Variable } & \multicolumn{1}{c|}{ Value } & \multicolumn{1}{c|}{ Reason } \\
\hline Dry-bulb temperature & See Table 5-3, Figure 5-7 & Driving function \\
\hline Wet-bulb temperature & As low as possible & $\begin{array}{l}\text { Reduces moisture effects on } \\
\text { infiltration heat loss }\end{array}$ \\
\hline Dew point & As low as possible & - \\
\hline Humidity ratio & As low as possible & $\begin{array}{l}\text { Reduces moisture effects on } \\
\text { infiltration heat loss }\end{array}$ \\
\hline Global horizontal radiation & 0 & No radiation \\
\hline Direct normal radiation & 0 & No radiation \\
\hline Diffuse radiation & 0 & No radiation \\
\hline Wind speed & 0 or constant & $\begin{array}{l}\text { Constant is chosen if wind } \\
\text { speed is used to set external } \\
\text { film coefficient }\end{array}$ \\
\hline Wind direction & & - \\
\hline
\end{tabular}

Table 5-3. Type A Weather Ambient Temperature

\begin{tabular}{|l|c|c|}
\hline \multirow{2}{*}{ Month } & \multicolumn{2}{|c|}{ Dry-Bulb Temperature } \\
\cline { 2 - 3 } & ${ }^{\circ} \mathbf{C}$ & ${ }^{\circ} \mathbf{F}$ \\
\hline January & -28.9 & -20 \\
\hline February & -6.7 & 20 \\
\hline March & 48.9 & 120 \\
\hline April & 48.9 & 120 \\
\hline May & 26.7 & 80 \\
\hline June & -28.9 & -20 \\
\hline July & -28.9 & -20 \\
\hline
\end{tabular}

\subsubsection{Type B Weather}

Type B weather is used with the tests that require step function changes in solar radiation levels. The weather variables used in Type B weather are described in Table 5-4 and Figure 5-8. The global horizontal (diffuse) radiation for January and March was zero, but February has a flux of $2776 \mathrm{~W} / \mathrm{m}^{2}$. 


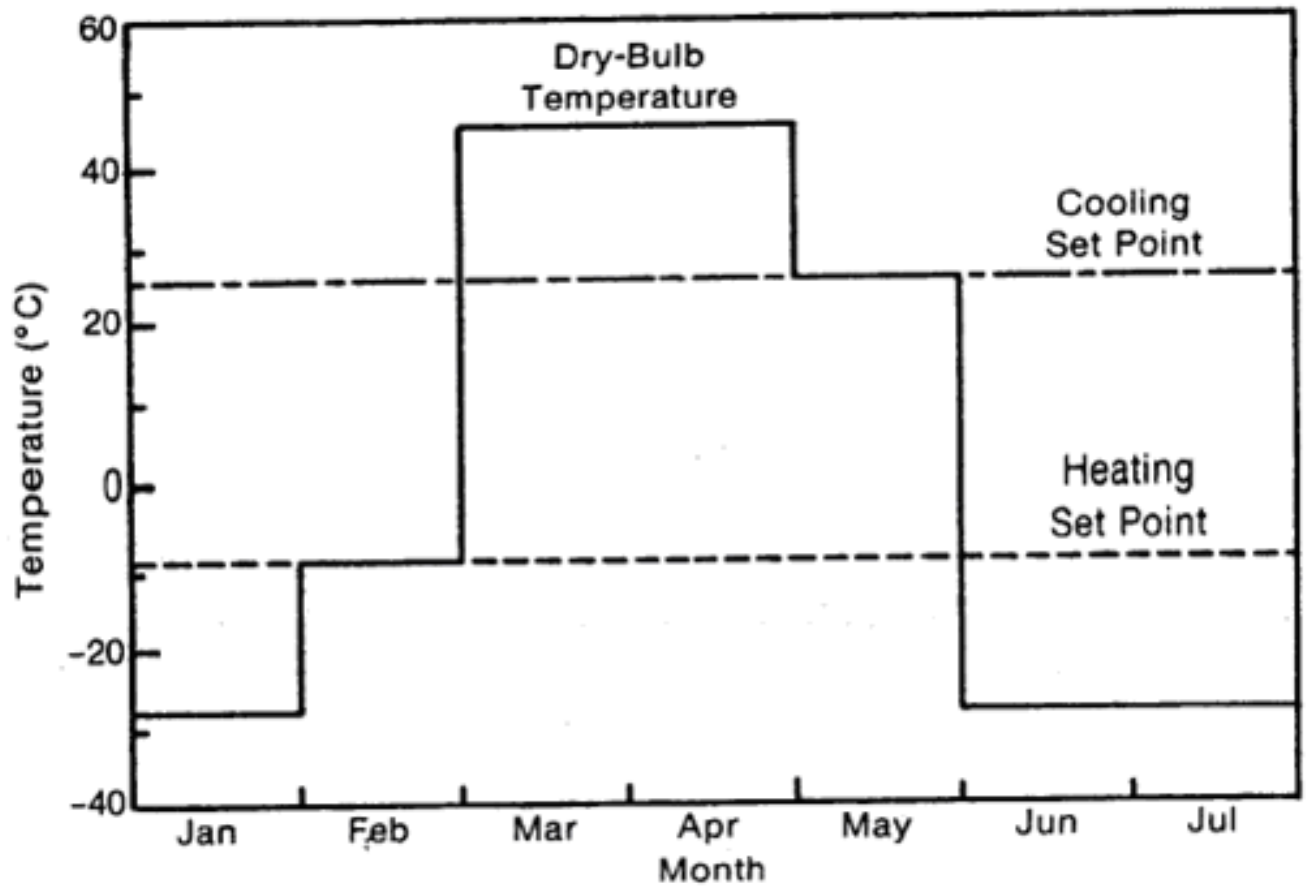

Figure 5-7. Dry-bulb temperature profile: temperature step function

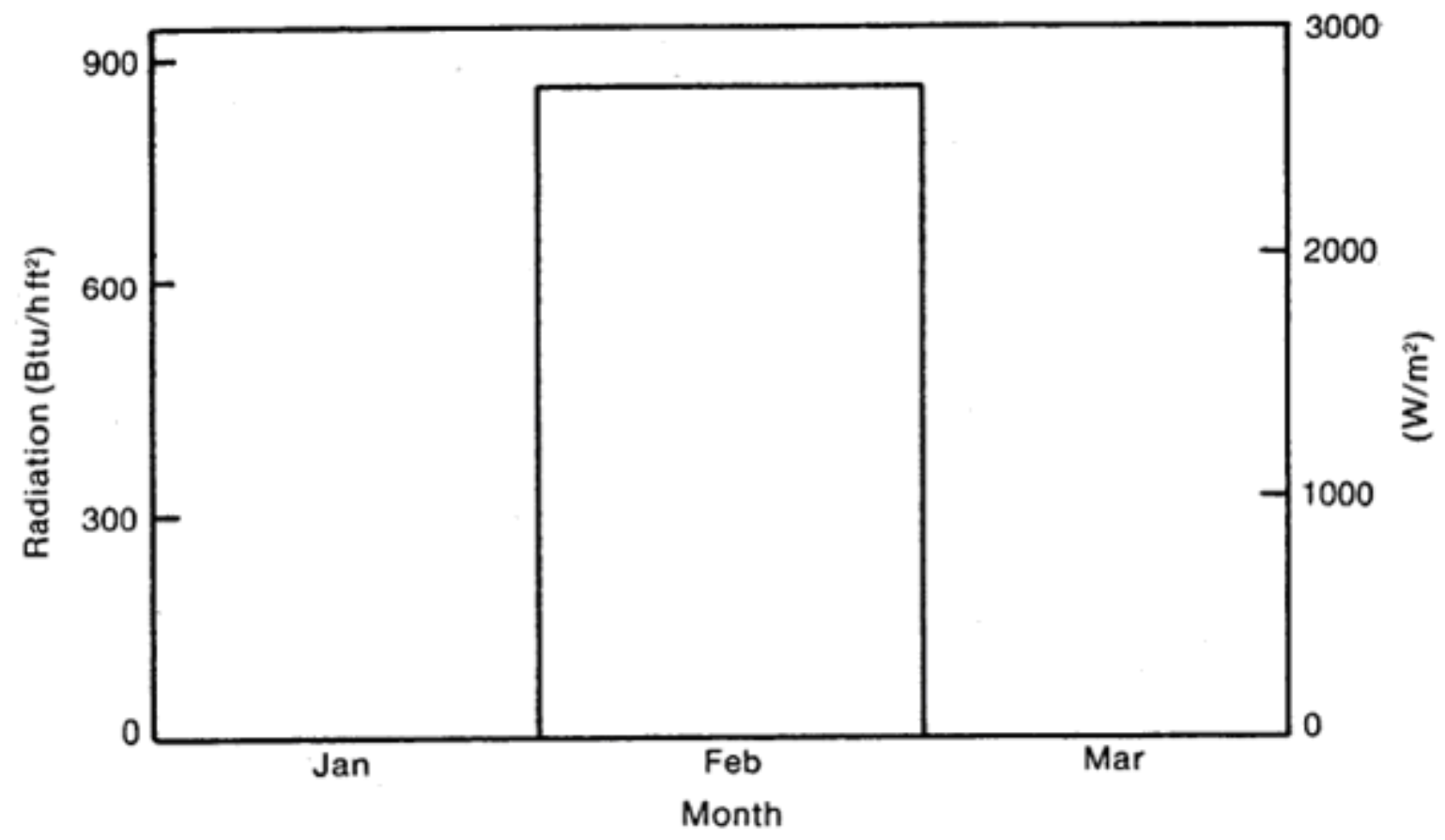

Figure 5-8. Total horizontal radiation step function 
Table 5-4. Type B Weather Data

\begin{tabular}{|l|l|l|}
\hline \multicolumn{1}{|c|}{ Variable } & \multicolumn{1}{c|}{ Value } & \multicolumn{1}{c|}{ Reason } \\
\hline Dry-bulb temperature & $26.7^{\circ} \mathrm{C}\left(80^{\circ} \mathrm{F}\right)$ & Reduces conductive heat loss \\
\hline Wet-bulb temperature & As low as possible & $\begin{array}{l}\text { Reduces moisture effects on } \\
\text { infiltration heat loss }\end{array}$ \\
\hline Dew point & As low as possible & - \\
\hline Humidity ratio & As low as possible & $\begin{array}{l}\text { Reduces moisture effects on } \\
\text { infiltration heat loss }\end{array}$ \\
\hline Global horizontal radiation & See Table 5-5, Figure 5-8 & Driving function \\
\hline Direct normal radiation & 0 & $\begin{array}{l}\text { Tests consider only diffuse } \\
\text { radiation }\end{array}$ \\
\hline Diffuse radiation & Global horizontal radiation & Driving function \\
\hline Wind speed & 0 or constant & $\begin{array}{l}\text { Constant is chosen if wind } \\
\text { speed is used to set external } \\
\text { film coefficient }\end{array}$ \\
\hline Wind direction & & - \\
\hline
\end{tabular}

\subsubsection{Use of the Weather Data}

The Type A and Type B weather data should be put into a format that is compatible with the simulation code to be tested and used as an input file when the various tests are performed. Most codes will readily accept synthetic weather data, but some can recognize that these data do not correspond to normal weather patterns and will not run them. In particular, these codes will not accept constant levels of solar radiation for month-long periods. These codes must be internally modified to deliver comparable solar radiation values to the building load portion of the code; this may require several changes to the code.

\subsection{Test Procedures}

The procedures needed to implement the analytical verification tests on a building energy analysis code are described in Appendix A. The instructions will include the following information:

- Test name

- Mechanisms tested

- Weather type

- Test building

- Test building description

- Procedure

- Interpretation of results

- Notes on implementation of the test.

Table 5-5 gives a description of the tests. 
Table 5-5. Description of the Tests

\begin{tabular}{|l|c|l|}
\hline \multicolumn{1}{|c|}{ Test } & $\begin{array}{c}\text { Weather and } \\
\text { Building Type }\end{array}$ & \multicolumn{1}{c|}{ Purpose } \\
\hline Low mass decay and steady-state loss & A & $\begin{array}{l}\text { Tests steady-state load/temperature } \\
\text { rise and decay }\end{array}$ \\
\hline Glazing & A & Determines UA of glazings \\
\hline Conservation & A & $\begin{array}{l}\text { Checks building responses caused by } \\
\text { changes in overall UA }\end{array}$ \\
\hline Infiltration & A & $\begin{array}{l}\text { Checks building responses caused by } \\
\text { variations in infiltration rate }\end{array}$ \\
\hline High mass & A & $\begin{array}{l}\text { Checks building responses caused by } \\
\text { variations in thermal mass }\end{array}$ \\
\hline Low-mass solar charging + window $\tau$ & B & $\begin{array}{l}\text { Tests window transmissivity and } \\
\text { temperature response of thermal mass } \\
\text { caused by solar radiation }\end{array}$ \\
\hline Glazing $\tau$ & B & $\begin{array}{l}\text { Tests window transmissivity for single } \\
\text { and triple glazings }\end{array}$ \\
\hline High-mass charging & B & $\begin{array}{l}\text { Checks temperature response of } \\
\text { thermal mass caused by solar radiation } \\
\text { as mass is varied }\end{array}$ \\
\hline
\end{tabular}

The procedure used to run a code through a test is as follows:

- Develop a building description input file that describes the building parameters listed in the test building and test building description sections. This step may require changes in the computer code. Read the "Notes on Implementation of the Test" with each test in Appendix A and Section 5.5 of this report for suggestions on these changes. If possible, run each test only with an appropriate building description input file and without coding changes.

- Use the appropriate weather input file to drive the simulation. The data contained in the weather file, either Type A or Type B, are described in Section 5.3. It may be necessary to change the coding of the simulation to use the synthetic weather data needed for these tests. However, it is best to run these tests without modification to the code.

- Run the simulation according to the instructions in the procedure step.

- Analyze the output of the simulation runs in accordance with the interpretation of the results step.

\subsection{Code Modifications}

The analytical verification technique procedures were originally designed to be implemented on the BEAS codes with no changes in the codes. The simple building models developed for the tests were chosen to be compatible with the input formats of the various simulations. However, implementation of the tests required that programming changes be made to certain BEAS to satisfy the conditions of the tests. These changes were highly code dependent and required extensive knowledge of the individual codes. The changes required to implement the tests on SUNCAT-2.4, DOE-2.1, DEROB 3.0, DEROB-4.0, and BLAST-3.0 are described and suggestions are made about changes that may be needed to implement these tests on other codes. 


\subsubsection{SUNCAT-2.4}

SUNCAT-2.4 is the simplest of the codes tested and required no internal code changes for the implementation of the analytical verification technique test procedures. The analogue circuit model used in SUNCAT-2.4, with no internal radiation network and user-controlled thermal resistances, is representative of many available simulations. This may mean that the technique can also be applied to a large number of simulations without programming changes in the codes.

\subsubsection{DOE-2.1}

DOE-2.1 is a complex code with many internal checks on input weather and building data. DOE2.1 required several programming changes, since it would not accept unusual weather data by the use of input alone. Many computer runs were required to ensure that the DOE-2.1 program accepted and responded to the proper weather and building description data for each test.

Implementation of the analytical verification tests on DOE-2.1 required the following modifications to the code:

- Disabled algorithms that check the validity of the input weather data.

- Hard-wired logical steps to ensure that input weather data are always used.

- Disallowed roof modeling because of a hard-wired, night sky radiation model in the code. Instead the roof area was accounted for by increasing the wall height. (This is not a change to the code, but rather an input.)

For the Type B weather tests, we made the following modifications:

- Disabled logic in the radiation processor so that solar radiation is not turned off at night.

- Installed isotropic sky model for diffuse radiation.

- Hard-wired diffuse radiation flux in both the weather processor and the loads program.

\subsubsection{DEROB-3 and DEROB-4}

Implementation of the analytical verification tests on DEROB-3 and DEROB-4 required the following changes:

- Hard-wired internal and external film coefficients to constant, selected values. The external "film" coefficient was modified to include the effects of the insulation specified in the various building descriptions.

- Disabled internal and external infrared exchange mechanisms by setting these infrared absorptivities to zero.

- Disabled the radiant absorptivity of the glazing material for the tests by using weather type B.

- Disabled the perimeter loss model in DEROB-3. 
The last item is an example of a mechanism included in a code that must be eliminated to set up the conditions for the analytical verification tests. Code developers should know if mechanisms of this type reside in their codes and how these mechanisms can be disabled.

\subsubsection{BLAST-3.0}

Implementation of the analytical verification tests on BLAST-3.0 required the following changes:

- Hard-wired internal film coefficients to the appropriate values.

- Used undocumented input statement to set tight convergence criteria for temperatures and loads.

\subsection{Test Results}

The analytical verification tests described in Appendix A were run on five building energy simulation codes: DOE-2.1, BLAST-3.0, SUNCAT-2.4, DEROB-3, and DEROB-4. The results of these runs are presented in this section, and deviations from the analytical solutions are noted and discussed.

We chose the codes used in this study for their potentially wide use in the building analysis industry and for their different modeling approaches. We found the entire testing procedure to be as much a learning process on how to modify and implement the analytical verification technique on a variety of codes as it is a test of the codes. We discovered the importance of modifying mechanisms in the simulation codes.

\subsubsection{Low-Mass Decay and Steady-State Heat Loss}

\subsubsection{Dynamic Results}

The dynamic results from Test 1 are presented in Figures 5-9 and 5-10. The temperature decay results in Figure 5-9 include the analytical solution as well as results from SUNCAT-2.4, DOE2.1, and BLAST-3.0. All three codes essentially agree with the analytical solution. However, no quantitative comparisons were performed. The DEROB-3 results in Figure 5-10 show a large deviation from the analytical solution. We pinpointed the cause of this deviation and attempted to correct it (see Section 5.6.1.2). 


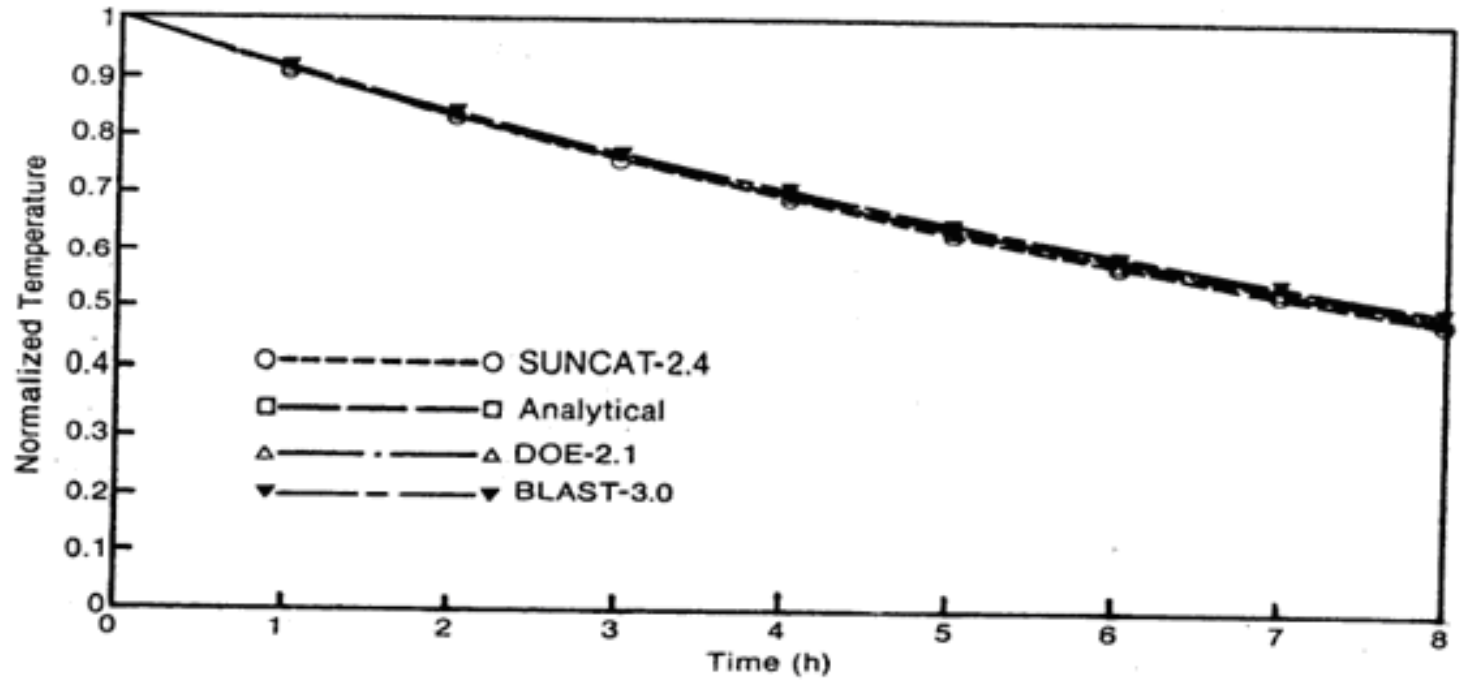

Figure 5-9. Low-mass decay test results-analogue solution

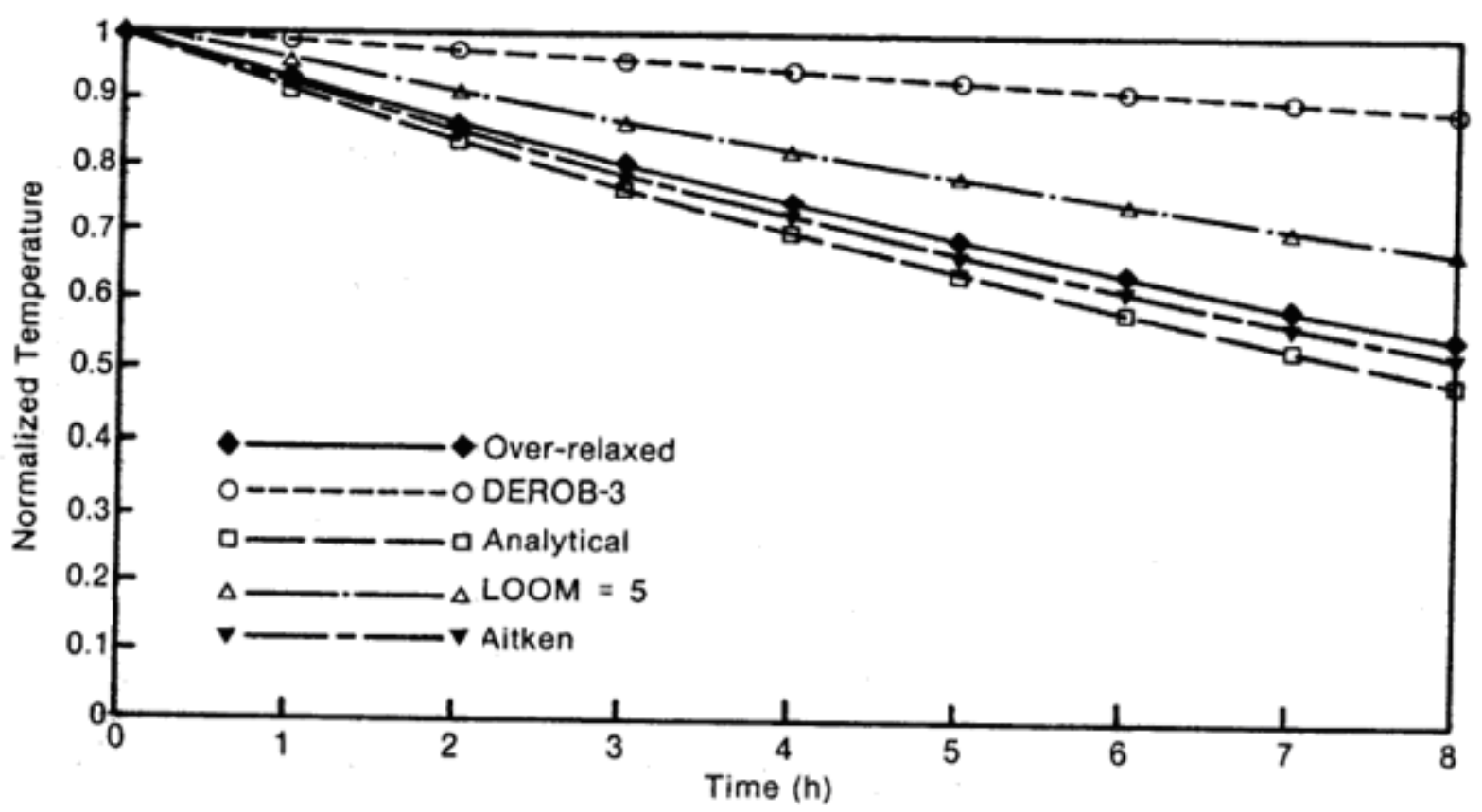

Figure 5-10. DEROB-3 fix study

The results from DEROB-4 are not included for this test. The DEROB-4 program cannot model the building used in this test. This program internally sets the internal node spacing through massive wall components. Because low-mass walls were used in this test, the simulation showed no nodes in the wall, or a zero mass wall. Since the decay of interior temperature is a function of the thermal mass of the building, a code that reads only zero mass walls will not produce accurate results.

A comparison of the low-mass decay results and the infinite series solution is shown in Figure 511. An investigation of outputs showed that the temperature rise and temperature decay were symmetric for all codes. 


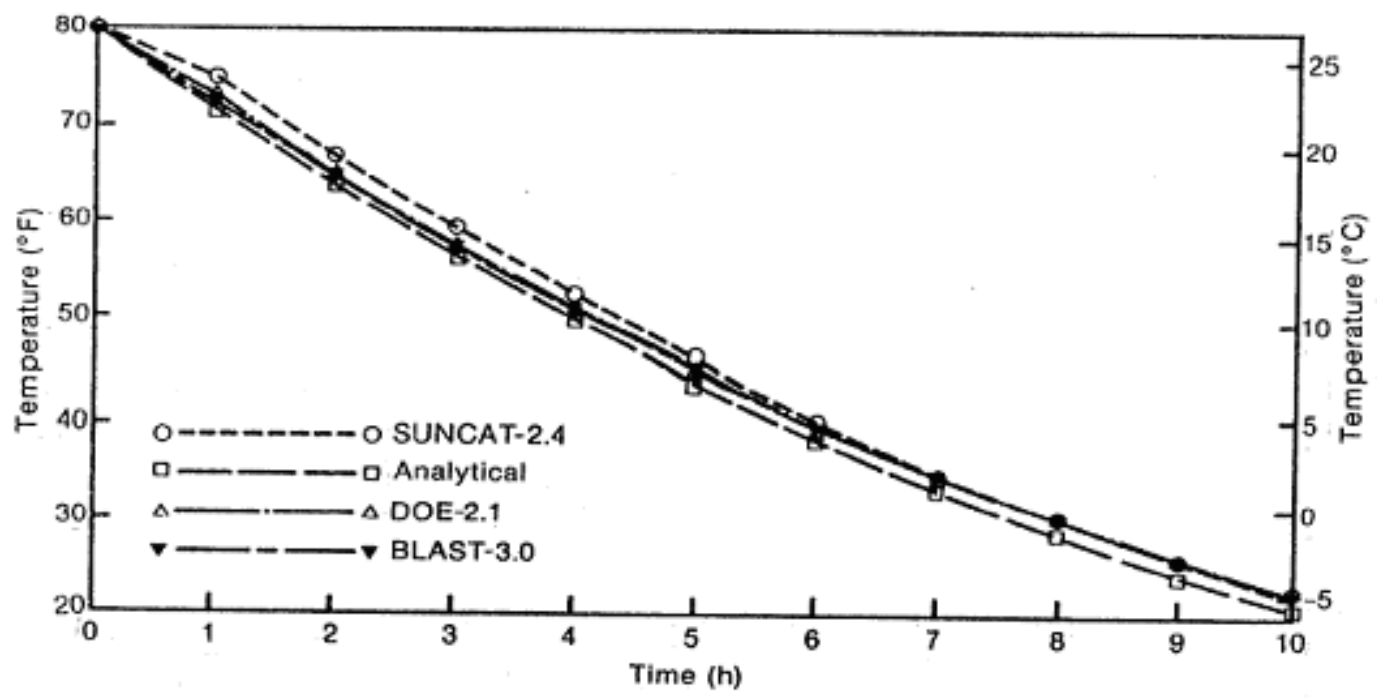

Figure 5-11. Low-mass decay test results-series solution

\subsubsection{Investigation of DEROB-3}

In the comparative study, the DEROB-3 program showed an incorrect sensitivity to changes in a building's thermal mass. That is, a wide range of building thermal mass produced only small changes in annual heating and cooling loads and daily temperature profiles. This behavior was investigated using the analytical verification tests described in this section.

The original DEROB-3 program showed a very slow temperature decay response when run through both the high-mass and low-mass decay tests (see Figure 5-10 for the low-mass decay results). Investigation of the code reveals that the numerical technique used to arrive at temperatures for each time step requires iterations for convergence to occur. However, the code was written so only one iteration is performed for each time step, apparently to reduce run time. The iteration routine is controlled by the variable LOOM, which is set equal to one in the original code. If several iterations are required for the convergence of the temperatures in a building, a single iteration will produce relatively small temperature differences between one time step and the next. Also, the convergence of all building component temperatures implies that the first law of thermodynamics has been satisfied and that an energy balance has been achieved. Failure to converge implies that the first law of thermodynamics has not been satisfied and that the energy that would be accounted for in the thermal mass temperature changes associated with convergence is not accounted for. In practical terms, this simulation projects annual heating loads characteristic of low-mass buildings and annual cooling loads characteristic of high-mass buildings. The annual heating loads are high because small thermal mass temperature changes allow little solar energy to be stored for nighttime use. Also, annual cooling loads are low because the small temperature changes in the mass reduce the effect of solar energy on the zone air temperature.

The temperature differences between one time step and the next should be greater for low-mass components than high-mass components. We decided that the low-mass case was therefore the more limiting case in DEROB-3. Thus, methods that would correct the problem in the low-mass case would also work for greater mass, and we used the low-mass case for our investigation. 
The most obvious way to increase the accuracy of the DEROB-3 program was to increase the number of iterations performed during each time step. Since an increase in the number of iterations is directly related to computer time and expense, and since DEROB-3 is a relatively expensive program to run, we decided that no more than five iterations per time step could be permitted. Code variable LOOM was set equal to 5, and the low-mass decay test was rerun. These results (Figure 5-10) still show significantly slower response than the analytical solution. Apparently, increasing the number of iterations by itself is not a solution to the problem in DEROB-3.

The next method to accelerate the temperature response of DEROB-3 is a numerical technique called Aitken's Method. This technique can be found in [3]. This method requires temperature information for at least three iterations in each time step. After many runs, we decided that this method would be used economically with LOOM set equal to 5. Figure 5-10 shows that this method gives a significantly more accurate performance than the first LOOM $=5$ case without increasing computer time.

A third technique used to increase the accuracy of the DEROB-3 program is called overrelaxation. In this technique, a relaxation coefficient is applied to certain factors in the surface node equations in the code. We determined that a relaxation coefficient of 1.5 and LOOM $=5$ produced the most economical application of this method. This approach produces a more accurate response than the first LOOM $=5$ test (see Figure 5-10), but it is less accurate than the Aitken method. Since all three methods use essentially the same amount of computer time, the Aitken approach is preferred.

The results from this investigation were sent to the author of DEROB-3. The author sent code corrections to us that incorporated the Aitken method and the number of iterations per time step determined by convergence criteria. This technique will require many iterations per time step when large temperature differences are experienced, such as when the sun is shining on a lowmass surface. However, time steps that have small temperature differences, such as high-mass surfaces at night, will need few iterations. Attempts to install these corrections on our version of DEROB-3 failed. However, we soon received an updated code, DEROB-4, that incorporated these corrections as well as other changes unrelated to the convergence problem.

\subsubsection{Steady-State Results}

The steady-state results for Test 1 are presented in Table 5-6. Again, DEROB-4 is not included. The results show excellent agreement with the analytical solution for total building heating loads. DEROB-3 is the furthest from the analytical solution, showing a 3\% greater heating load. This variance can be explained by the use of a high thermal resistance floor for this code instead of an adiabatic one. This floor would add about $3 \%$ to the overall building load coefficient. The building cooling load coefficients are essentially the same as the heating load coefficients. 
Table 5-6. Low- and Mid-Mass Steady-State Results

(in $\mathrm{W} /{ }^{\circ} \mathrm{C}\left[\mathrm{Btu} / \mathrm{h}^{\circ} \mathrm{F}\right]$ )

\begin{tabular}{|l|c|c|c|c|c|c|}
\hline & Analytical & SUNCAT-2.4 & DOE-2.1 & BLAST 3.0 & DEROB-3 & DEROB-4 \\
\hline \multirow{2}{*}{ Low-mass } & 26.38 & 26.73 & 26.38 & 26.41 & 27.09 & - \\
& $(50.0)$ & $(50.67)$ & $(50.0)$ & $(50.07)$ & $(51.36)$ & - \\
\hline \multirow{2}{*}{ Mid-mass UA } & 26.36 & - & - & 26.41 & - & 26.39 \\
& $(50.0)$ & - & - & $(50.06)$ & - & $(50.03)$ \\
\hline
\end{tabular}

\subsubsection{Mid-Mass Decay Test}

The mid-mass decay test was run on BLAST-3.0 and DEROB-4 only. The results are presented in Figures 5-12 and 5-13 and Table 5-6. Both the dynamic and steady-state results are essentially identical to the analytical solutions.

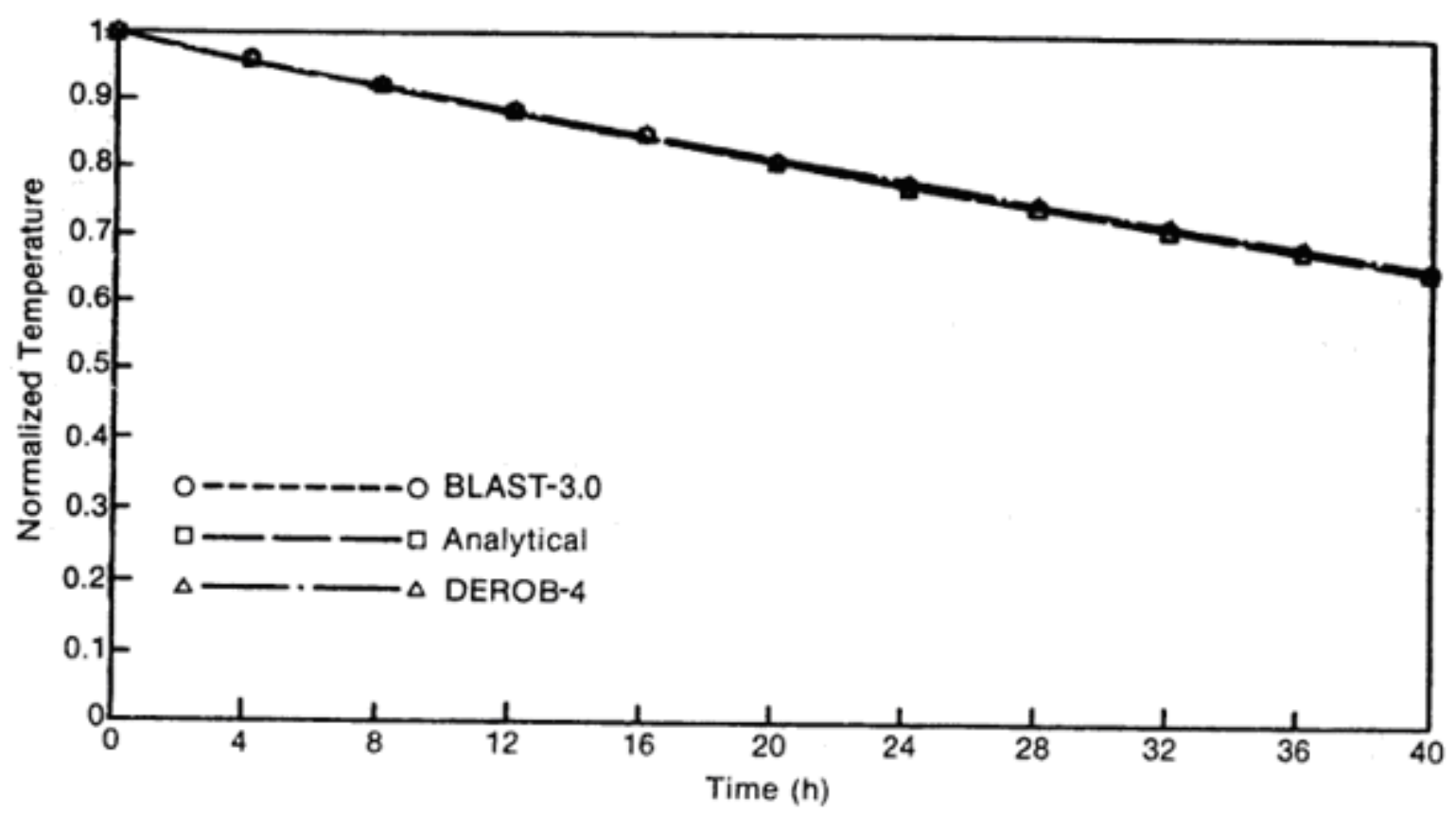

Figure 5-12. Mid-mass decay test results-analogue solution 


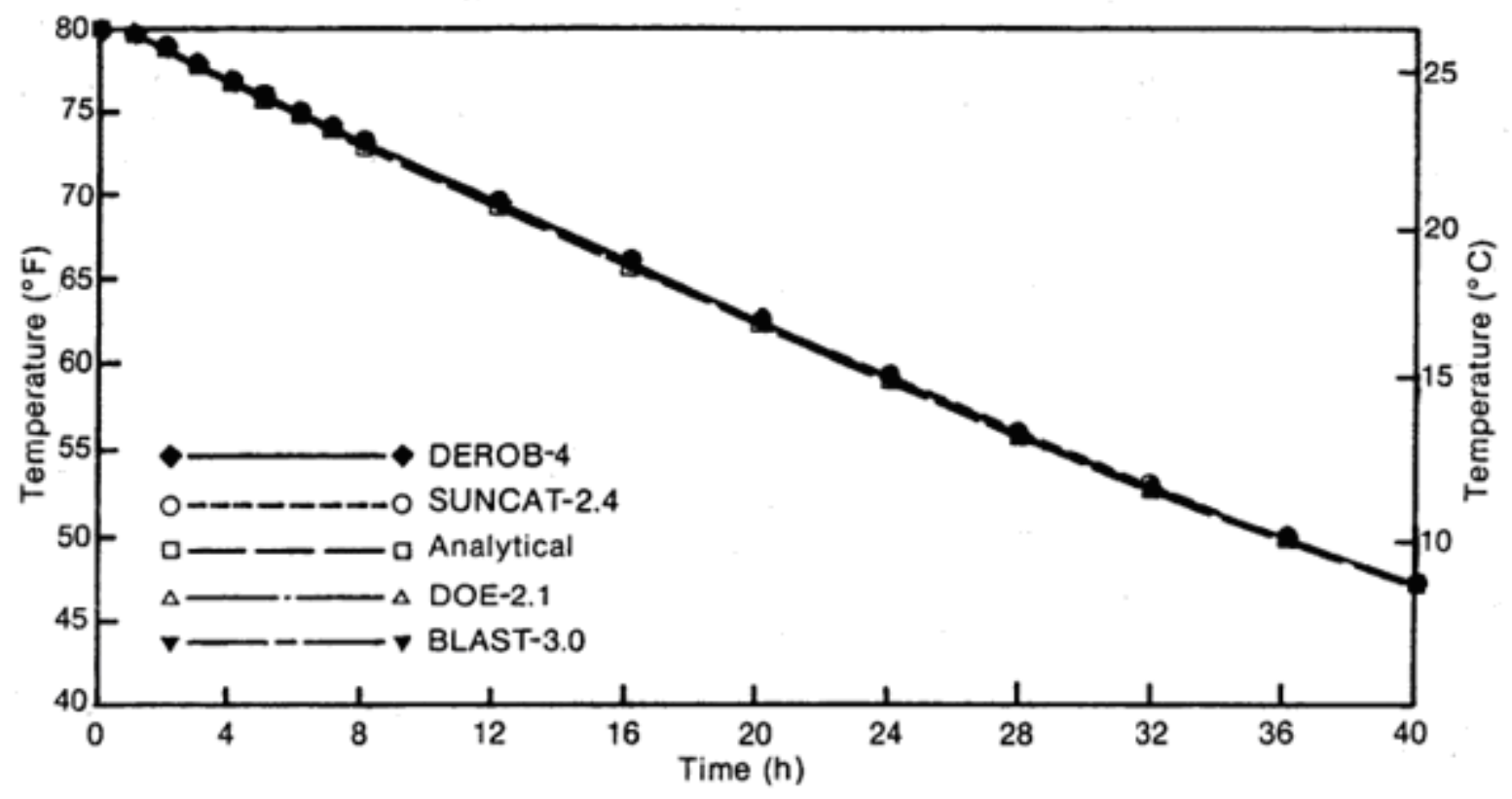

Figure 5-13. Mid-mass temperature decay results-exact solution

\subsubsection{High-Mass Decay Test}

The high-mass decay test was run on all five codes, and the results from all except DEROB-3 are presented in Figures 5-14 and 5-15 and Table 5-7. The codes produce results extremely close to the dynamic and steady-state analytical solutions.

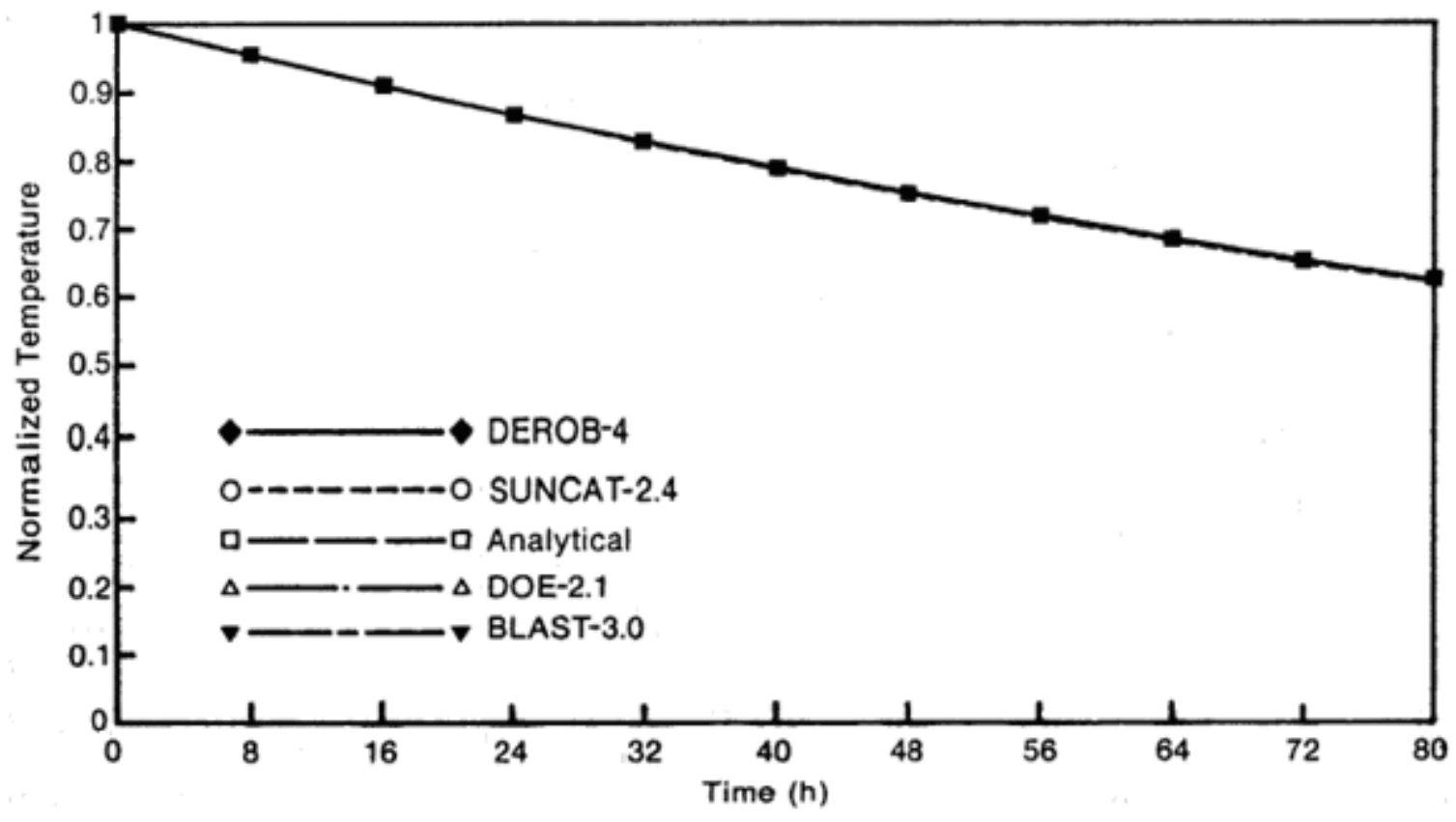

Figure 5-14. High-mass decay text-analogue solution 


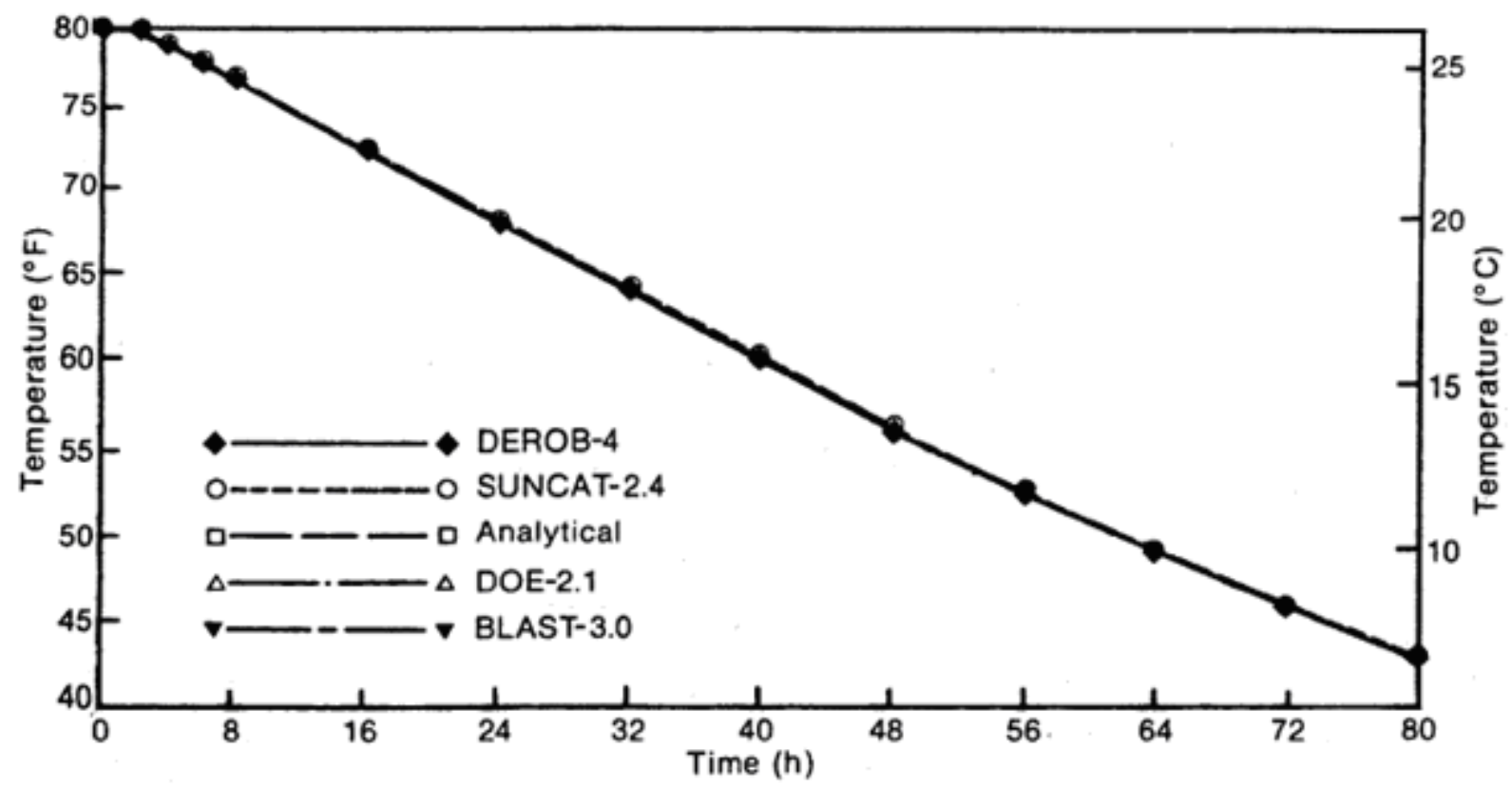

Figure 5-15. High-mass decay test-exact solution

Table 5-7. High-Mass Steady-State Results (in $\mathrm{W} /{ }^{\circ} \mathrm{C}\left[\mathrm{Btu} / \mathrm{h}{ }^{\circ} \mathrm{F}\right]$ )

\begin{tabular}{|c|c|c|c|c|c|}
\hline Analytical & SUNCAT-2.4 & DOE-2.1 & BLAST 3.0 & DEROB-3 & DEROB-4 \\
\hline 26.38 & 26.38 & 26.36 & 26.40 & 27.04 & 26.39 \\
$(50.0)$ & $(50.0)$ & $(49.96)$ & $(50.05)$ & $(51.25)$ & $(50.03)$ \\
\hline
\end{tabular}

\subsubsection{Glazing Test}

The glazing test was run on all five codes (see Figure 5-16). This test determines what assumptions were used for the window U-values in each code.

There is a wide range of window U-values for each number of glazings that can be attributed to at least two factors. First, for all of the codes except SUNCAT-2.4, the window descriptions provided by the code authors were used.

Thus, a double-glazed window in BLAST-3.0 is not necessarily the same as one in DEROB-4. In fact, there is apparently a large discrepancy between the internal window descriptions in DEROB-3 and DEROB-4. SUNCAT-2.4 uses an input U-value for glazing surfaces, and these were accurately reproduced in the output.

The second origin of the differences between the predicted window U-values is the different methods needed to establish known external film coefficients for the wall surfaces. These methods are summarized in Table 5-8. Since the external film coefficient is a major component of the overall conductance of the window, a range of window conductances should result. A code's internal algorithm should determine the value of the window external film coefficients. However, in most codes, this value is equal to the external film coefficient for the surface in which the window lies. Allowing the window to take on its own value for external film coefficient would require changes in each code. These were not done for DOE-2.1, BLAST-3.0, or either DEROB Program. 


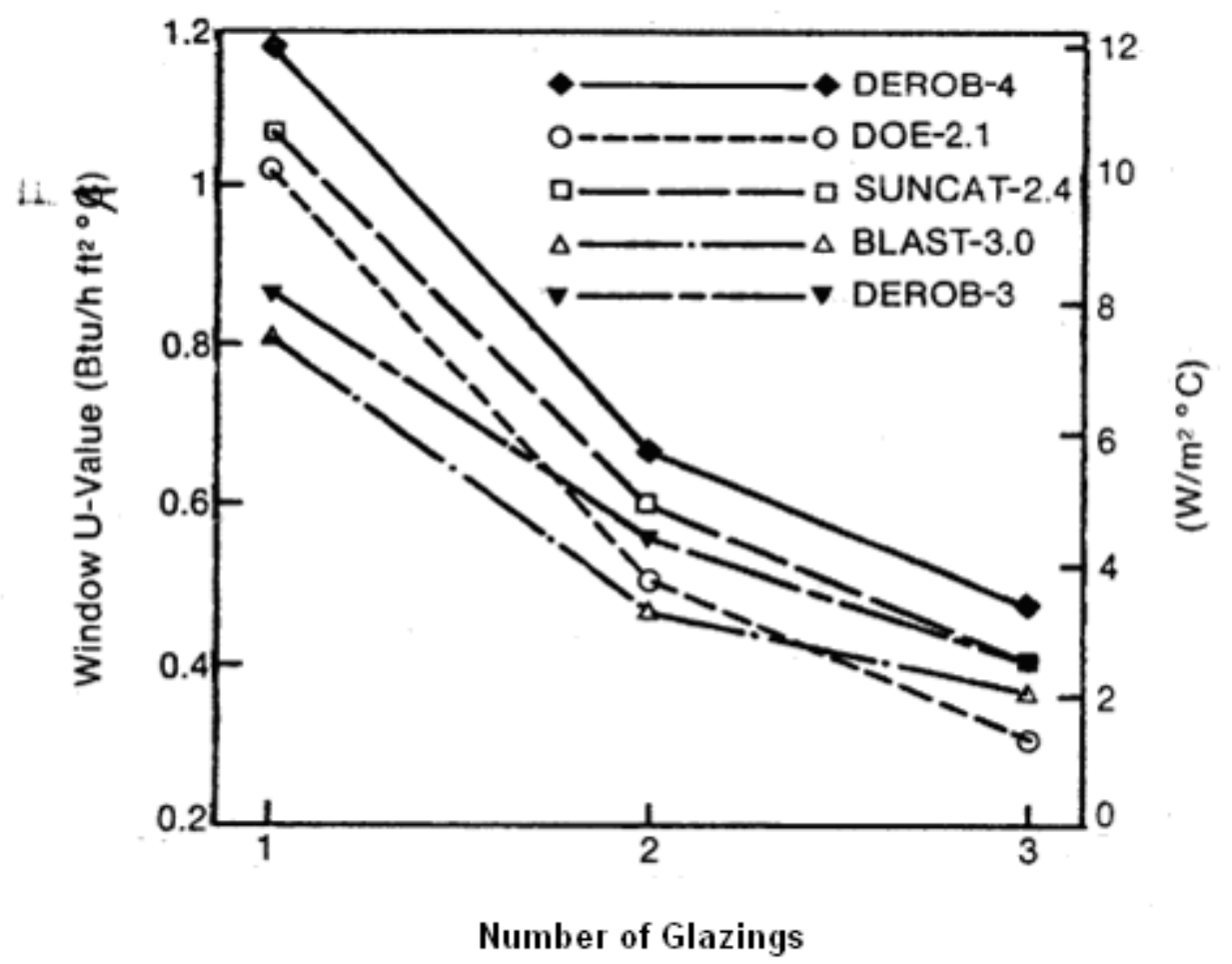

Figure 5-16. Glazing test results

Table 5-8. Methods Needed to Establish Known External Film Coefficients

\begin{tabular}{|l|l|}
\hline \multicolumn{1}{|c|}{ Code } & \multicolumn{1}{c|}{ Changes Needed to Set External Film Coefficient } \\
\hline SUNCAT-2.4 & None \\
\hline DOE-2.1 & Wind speed set at $3.6 \mathrm{~m} / \mathrm{s}(11.8 \mathrm{ft} / \mathrm{s})$ in weather file \\
\hline BLAST-3.0 & Other side coefficient set at $22.7 \mathrm{~W} / \mathrm{m}^{2}{ }^{\circ} \mathrm{C}\left(4.0 \mathrm{Btu} / \mathrm{h} / \mathrm{ft}^{2}{ }^{\circ} \mathrm{F}\right)$ \\
\hline DEROB-3 4 & External film coefficient hard-wired in code \\
\hline & \multicolumn{1}{|c|}{ Effects on Window External Film Coefficient } \\
\hline SUNCAT-2.4 & None \\
\hline DOE-2.1 & Windows always exposed to this wind speed \\
\hline BLAST-3.0 & External film coefficient always $22.7 \mathrm{~W} / \mathrm{m}^{2}{ }^{\circ} \mathrm{C}\left(4.0 \mathrm{Btu} / \mathrm{h} / \mathrm{ft}^{2}{ }^{\circ} \mathrm{F}\right)$ \\
\hline DEROB-3 and 4 & Windows always exposed to zero wind speed \\
\hline
\end{tabular}

\subsubsection{Conservation Test}

The conservation test was run on all five codes. Only steady-state results will be presented here (see Table 5-9.) All of the codes except DEROB-3 produced building load coefficients within $1 \%$ of the $10.55 \mathrm{~W} /{ }^{\circ} \mathrm{C}\left(20 \mathrm{Btu} / \mathrm{h}{ }^{\circ} \mathrm{F}\right)$ expected for the more heavily insulated building. DEROB-3 was $5 \%$ higher than the expected value. This again can be explained by the nonadiabatic floor input into this code.

The codes produced more variable results from the poorly insulated $79.13 \mathrm{~W} /{ }^{\circ} \mathrm{C}\left(150 \mathrm{Btu} / \mathrm{h}{ }^{\circ} \mathrm{F}\right)$ building. SUNCAT-2.4 was almost 4\% higher, DEROB-3 was more than $4 \%$ higher, and DEROB-4 was more than $12 \%$ higher than the expected value. We have not investigated the causes of these differences. 
Table 5-9. Conservation Test Steady-State Results

$\left(\mathrm{W} /{ }^{\circ} \mathrm{C}\left[\mathrm{Btu} / \mathrm{h}^{\circ} \mathrm{F}\right]\right)$

\begin{tabular}{|l|c|c|c|c|c|c|}
\hline & Analytical & SUNCAT-2.4 & DOE-2.1 & BLAST 3.0 & DEROB-3 & DEROB-4 \\
\hline Heavily insulated & 10.55 & 10.60 & 10.50 & 10.58 & 11.08 & 10.66 \\
building & $(20.0)$ & $(20.10)$ & $(19.90)$ & $(20.05)$ & $(21.00)$ & $(20.20)$ \\
\hline Poorly insulated & 79.13 & 82.13 & 79.71 & 79.18 & 82.5 & 88.94 \\
building & $(150.0)$ & $(155.7)$ & $(151.1)$ & $(150.1)$ & $(156.4)$ & $(168.6)$ \\
\hline
\end{tabular}

\subsubsection{Infiltration Test}

The infiltration test was run on all five codes, and the results are presented in Figure 5-17 and Table 5-10. All codes show a linear relationship between building UA and infiltration rate. The slope of this curve equals, with proper conversion, the volumetric specific heat of the infiltration air in $\mathrm{kJ} / \mathrm{m}^{3}{ }^{\circ} \mathrm{C}\left(\mathrm{Btu} / \mathrm{ft}^{3}{ }^{\circ} \mathrm{F}\right)$. Table 5-10 also shows that the slopes for SUNCAT-2.4 and DEROB-3 and 4 are very close to the specific heat value of $1.21 \mathrm{~kJ} / \mathrm{m}^{3}{ }^{\circ} \mathrm{C}\left(0.02 \mathrm{Btu} / \mathrm{ft}^{3}{ }^{\circ} \mathrm{F}\right)$ calculated using ideal gas assumptions for dry air at standard atmospheric pressure and at $21^{\circ} \mathrm{C}$ $\left(70^{\circ} \mathrm{F}\right)($ see Table $5-11)$.

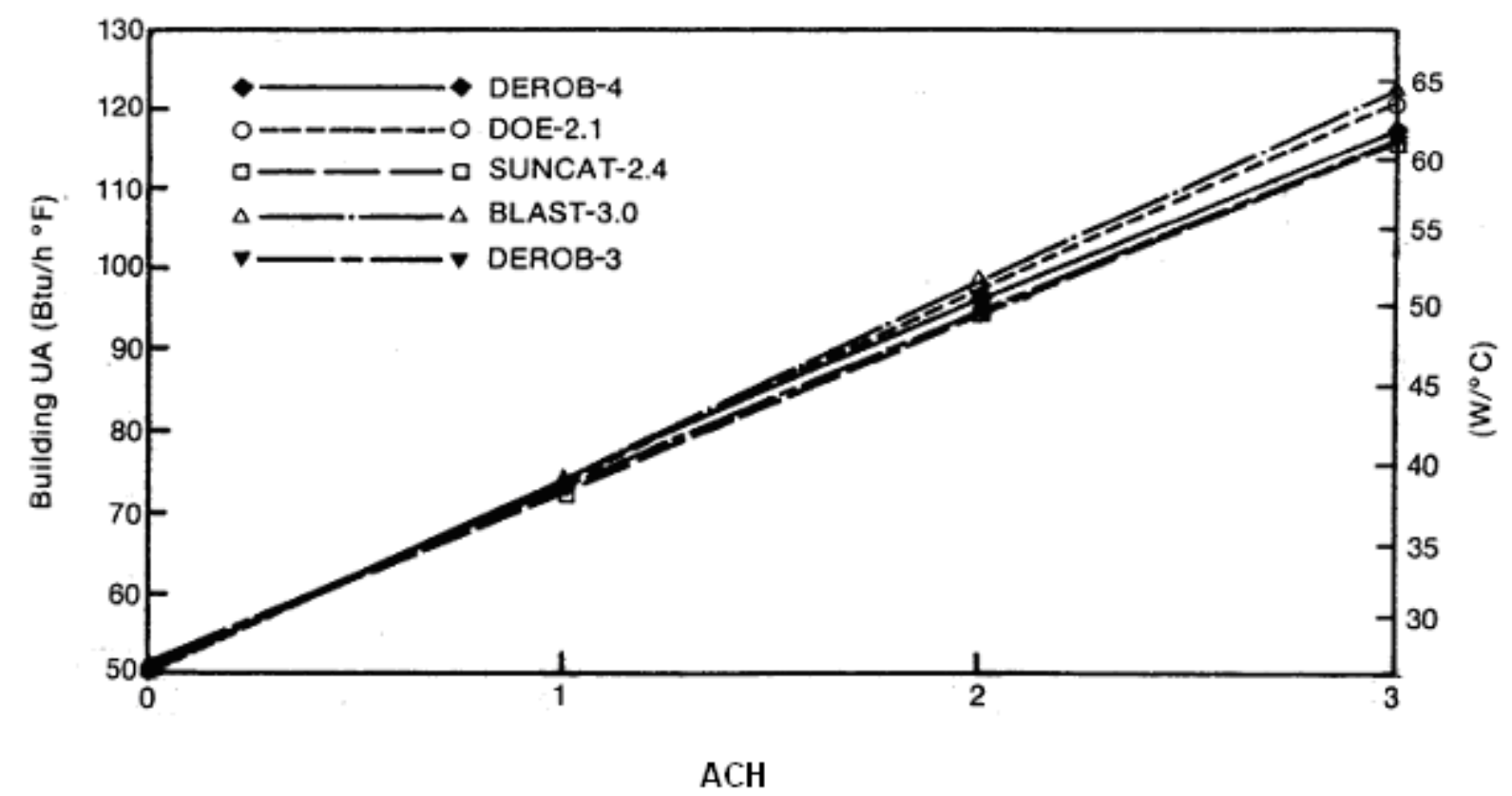

Figure 5-17. Infiltration test results 
Table 5-10. Infiltration Test Steady-State Results Building UA

$\left(\mathrm{W} /{ }^{\circ} \mathrm{C}\left[\mathrm{Btu} / \mathrm{h}^{\circ} \mathrm{F}\right]\right)$

\begin{tabular}{|c|c|c|c|c|c|}
\hline $\mathrm{ACH}$ & SUNCAT-2.4 & DOE-2.1 & BLAST 3.0 & DEROB-3 & DEROB-4 \\
\hline 0.5 & $\begin{array}{c}32.49 \\
(61.59)\end{array}$ & $\begin{array}{c}32.62 \\
(61.83)\end{array}$ & - & $\begin{array}{c}32.81 \\
(62.20)\end{array}$ & - \\
\hline 1 & $\begin{array}{c}38.25 \\
(72.50)\end{array}$ & $\begin{array}{c}38.88 \\
(73.71)\end{array}$ & $\begin{array}{c}39.15 \\
(74.22)\end{array}$ & $\begin{array}{c}38.51 \\
(73.01)\end{array}$ & $\begin{array}{c}39.24 \\
(74.38)\end{array}$ \\
\hline 1.5 & $\begin{array}{c}44.01 \\
(83.43)\end{array}$ & $\begin{array}{c}45.17 \\
(85.63)\end{array}$ & - & $\begin{array}{c}44.82 \\
(83.82)\end{array}$ & - \\
\hline 2 & $\begin{array}{c}49.76 \\
(94.33)\end{array}$ & $\begin{array}{c}51.40 \\
(97.44)\end{array}$ & $\begin{array}{c}51.88 \\
(98.34)\end{array}$ & $\begin{array}{c}49.93 \\
(94.64)\end{array}$ & $\begin{array}{c}50.63 \\
(95.98)\end{array}$ \\
\hline 3 & $\begin{array}{c}61.28 \\
(116.17)\end{array}$ & $\begin{array}{c}63.87 \\
(121.08)\end{array}$ & $\begin{array}{c}64.62 \\
(122.49)\end{array}$ & $\begin{array}{c}61.33 \\
(116.26)\end{array}$ & $\begin{array}{c}62.04 \\
(117.6)\end{array}$ \\
\hline 5 & $\begin{array}{c}84.31 \\
(159.83)\end{array}$ & $\begin{array}{c}88.91 \\
(168.54)\end{array}$ & - & $\begin{array}{c}84.16 \\
(159.53)\end{array}$ & - \\
\hline $\begin{array}{c}\text { Slope }\left(\mathrm{kJm}^{3}{ }^{\circ} \mathrm{C}\right) \\
\left(\mathrm{Btu} \mathrm{ft}^{3}{ }^{\circ} \mathrm{F}\right)\end{array}$ & $\begin{array}{c}1.221 \\
(0.0182)\end{array}$ & $\begin{array}{c}1.328 \\
(0.0198)\end{array}$ & $\begin{array}{c}1.348 \\
(0.0201)\end{array}$ & $\begin{array}{c}1.207 \\
(0.0180)\end{array}$ & $\begin{array}{c}1.207 \\
(0.0180)\end{array}$ \\
\hline
\end{tabular}

Table 5-11. Volumetric Specific Heat

\begin{tabular}{|c|c|c|c|}
\hline \multicolumn{2}{|c|}{ Temperature } & \multicolumn{2}{c|}{ Volumetric Specific Heat } \\
\hline${ }^{\circ} \mathbf{C}$ & ${ }^{\circ} \mathbf{F}$ & $\mathbf{C = ~} \mathbf{~ J /} / \mathbf{m}^{3}{ }^{\circ} \mathbf{C}$ & $\mathbf{C}=\mathbf{B t u} / \mathbf{f t}^{3}{ }^{\circ} \mathbf{F}$ \\
\hline-28.9 & -20 & 1.45 & 0.0216 \\
\hline-17.8 & 0 & 1.39 & 0.0207 \\
\hline-6.7 & 20 & 1.33 & 0.0198 \\
\hline 15.6 & 60 & 1.23 & 0.0183 \\
\hline 21.1 & 70 & 1.21 & 0.0180 \\
\hline 26.7 & 80 & 1.18 & 0.0176 \\
\hline
\end{tabular}

The volumetric specific heat $\mathrm{C}$ is calculated in the following manner:

$$
\mathrm{C}=\mathrm{C}_{\mathrm{p}} / \mathrm{V}
$$

Where,

$$
\begin{array}{lll}
\mathrm{C}_{\mathrm{p}} & = & \text { constant pressure specific heat of dry air } 1.0035 \mathrm{~kJ} / \mathrm{kg} \mathrm{K}\left(0.240 \mathrm{Btu} / \mathrm{lb}{ }^{\circ} \mathrm{F}\right) \\
\mathrm{V} & = & \text { specific volume of dry air }\left(\mathrm{R}_{\mathrm{a}}\right)(\mathrm{T}) / \mathrm{P} \\
\mathrm{R}_{\mathrm{a}} & = & \text { gas constant for air }=0.287 \mathrm{~kJ} / \mathrm{kg}{ }^{\circ} \mathrm{C}\left(53.35 \mathrm{ft} \cdot \mathrm{lb} / \mathrm{lb}{ }^{\circ} \mathrm{F}\right) \\
\mathrm{T} & = & \text { temperature in } \mathrm{K}\left({ }^{\circ} \mathrm{F}\right) \\
\mathrm{P} & = & \text { pressure }=101.33 \mathrm{kPa}\left(14.7 \mathrm{lbf} / \mathrm{in}^{2}\right)
\end{array}
$$

Therefore,

$$
\begin{aligned}
\mathrm{v} & =(0.287 \mathrm{~kJ} / \mathrm{kg} \mathrm{K})(\mathrm{T}) / 101.33 \mathrm{kPa} \\
& =\left[2.83 \times 10 \mathrm{~m}^{3} /(\mathrm{kg} \mathrm{K})\right] \mathrm{T}
\end{aligned}
$$

Therefore,

$$
\mathrm{C}=\mathrm{Cp} / \mathrm{V}=\left(354.3 \mathrm{~kJ} / \mathrm{m}^{3}\right)(1 / \mathrm{T}) \text {. }
$$

However, since this heating test is run with an indoor and ambient temperature of $-6.7^{\circ} \mathrm{C}\left(20^{\circ} \mathrm{F}\right)$, the volumetric specific heat should be $1.33 \mathrm{~kJ} / \mathrm{m}^{2}{ }^{\circ} \mathrm{C}\left(0.02 \mathrm{Btu} / \mathrm{ft}{ }^{\circ} \mathrm{F}\right)$. The results from DOE-2.1 and BLAST-3.0 are near this value. Thus, these codes apparently calculate the correct value of 
volumetric specific heat to use for air infiltration heat exchange. The other three codes use a simplified algorithm that assumes that both room and ambient air are at about $21^{\circ} \mathrm{C}\left(70^{\circ} \mathrm{F}\right)$. This difference should lead to lower predicted heating loads caused by infiltration when the results from SUNCAT-2.4, DEROB-3, or DEROB-4 are compared to those from BLAST-3.0 and DOE2.1. However, this should not be a problem when heating set point temperatures of about $21^{\circ} \mathrm{C}$ $\left(70^{\circ} \mathrm{F}\right)$ are used.

\subsubsection{Solar Charging and Window Transmissivity}

The glazing charging test was run on all five codes for window transmissivity and BLAST-3.0, DOE-2.1, and SUNCAT-2.4 for low-mass charge. The results for window transmissivity for diffuse radiation are shown in Figure 5-18 and those from the charging of low mass by solar radiation are shown in Figure 5-19. This test checks the assumptions on the window diffuse transmissivity parameter that are in each code.

The window transmissivity results show surprisingly large differences for the different codes, particularly for double- and triple-glazed windows. In this test, the code entry for a normal single-, double-, or triple-glazed window was used (except in SUNCAT). These entries are the default glazing variables that most code users would include in a building input file. Thus, a glazing used in DOE-2.1 or BLAST-3.0 would transmit significantly more diffuse solar radiation than the same type used for either DEROB program, particularly for double or triple glazing. Therefore, buildings with a great deal of direct gain window area should, on the basis of transmitted solar radiation, have lower annual heating loads and higher annual cooling loads on BLAST-3.0 and DOE-2.1 than on the DEROB codes. Note that the results for BLAST-3.0 and DOE-2.1 show close agreement for all three glazing numbers.

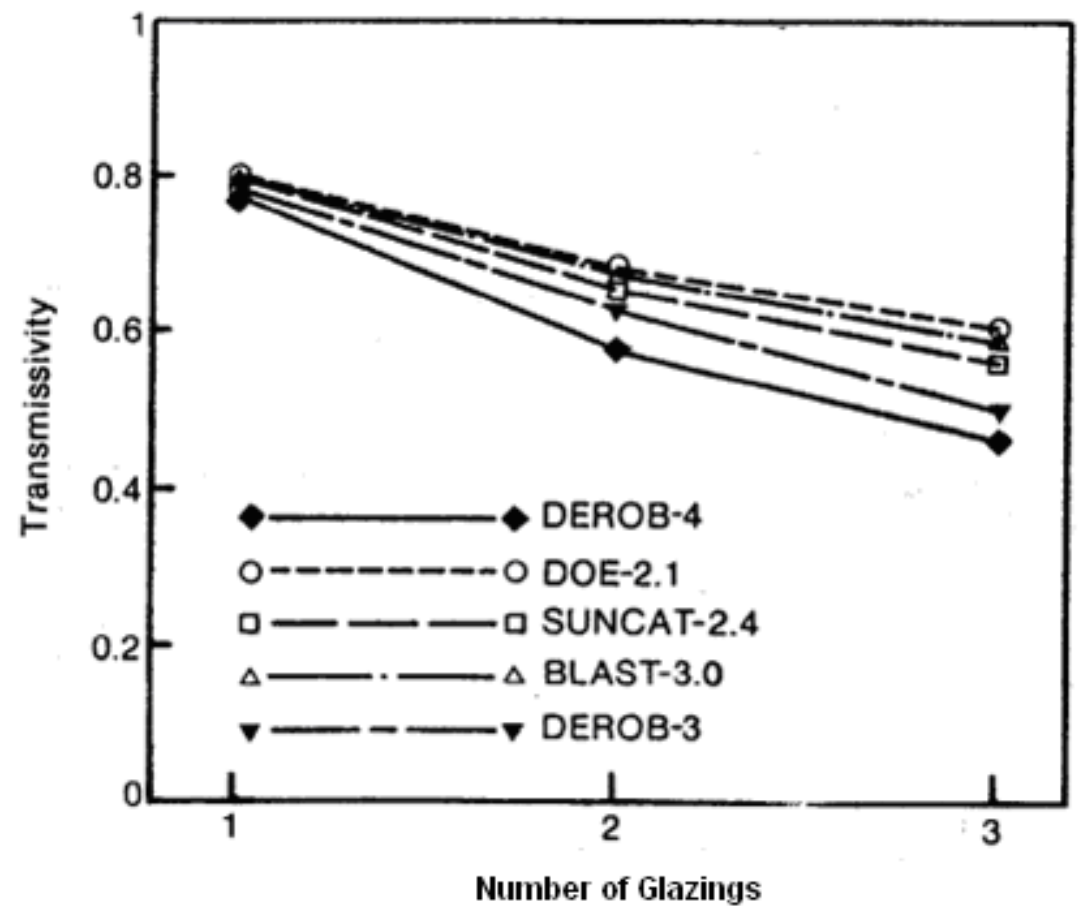

Figure 5-18. Glazing transmissivity test results 


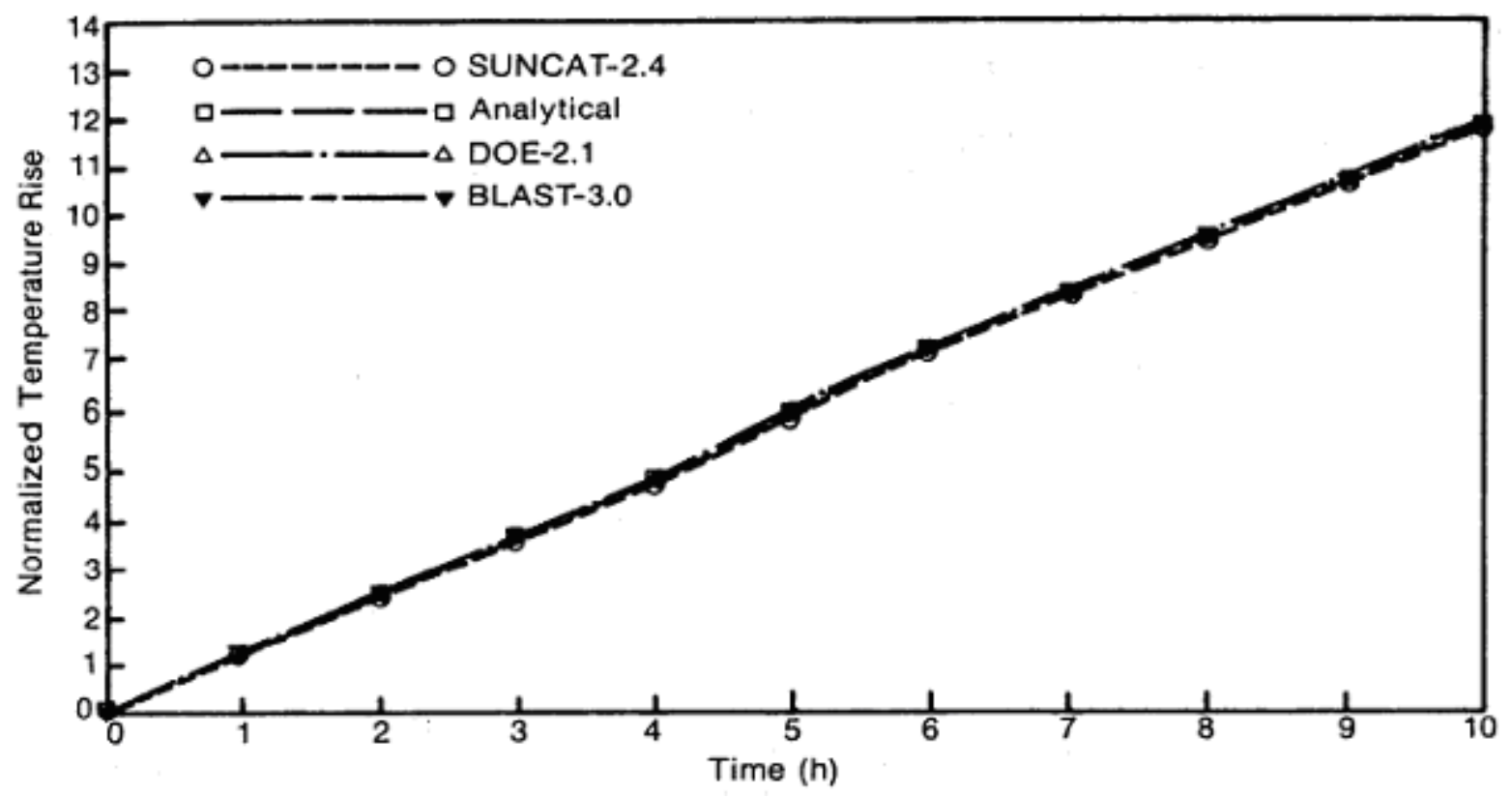

Figure 5-19. Low-mass charge test results

SUNCAT-2.4 was input by specifying the optical and physical properties of the glazing material. These values were chosen to correspond to the properties found in normal window glass. The results from SUNCAT show a lower diffuse transmissivity than DOE-2.I or BLAST-3.0, for all three levels of glazing. However, the differences are much smaller than between the DEROB codes and these two codes.

The loss-mass charge test results are shown in Figure 5-19. This test was not run on DEROB-4, since this code generates zero-mass wall surfaces for the low-mass wall construction. DOE-2.1, BLAST-3.0, and SUNCAT-2.4 show close agreement with the analytical solution. DOE-2.1 shows a slightly faster and SUNCAT-2.4 a slower temperature rise in the first hour than the analytical solution.

\subsubsection{Mid-Mass Charging Test}

The mid-mass charging test was run on DOE-2.1, BLAST-3.0, and DEROB-4, and the results are shown in Figure 5-20. DOE-2.1 showed a temperature rise faster than the analytical solution for the first four hours and comparable to the analytical solution thereafter. The other two codes show excellent agreement with the analytical solution.

\subsubsection{High-Mass Charging Test}

The high-mass charging test was run on DEROB-4, and the results are shown in SUNCAT-2.4, DOE-2.1, BLAST-3.0, and Figure 5-21. DOE-2.1 showed the same accelerated temperature rise as in the low- and mid-mass charging tests. It also shows about a 5\% greater slope in the linear portion (after hour 10) of the temperature rise, equivalent to a 5\% reduction in thermal mass utilized in this code compared with the other codes and the analytical solution. However, this slope difference does not appear in the results for low- and mid-mass levels. The other three codes show very close agreement with the analytical solution. 


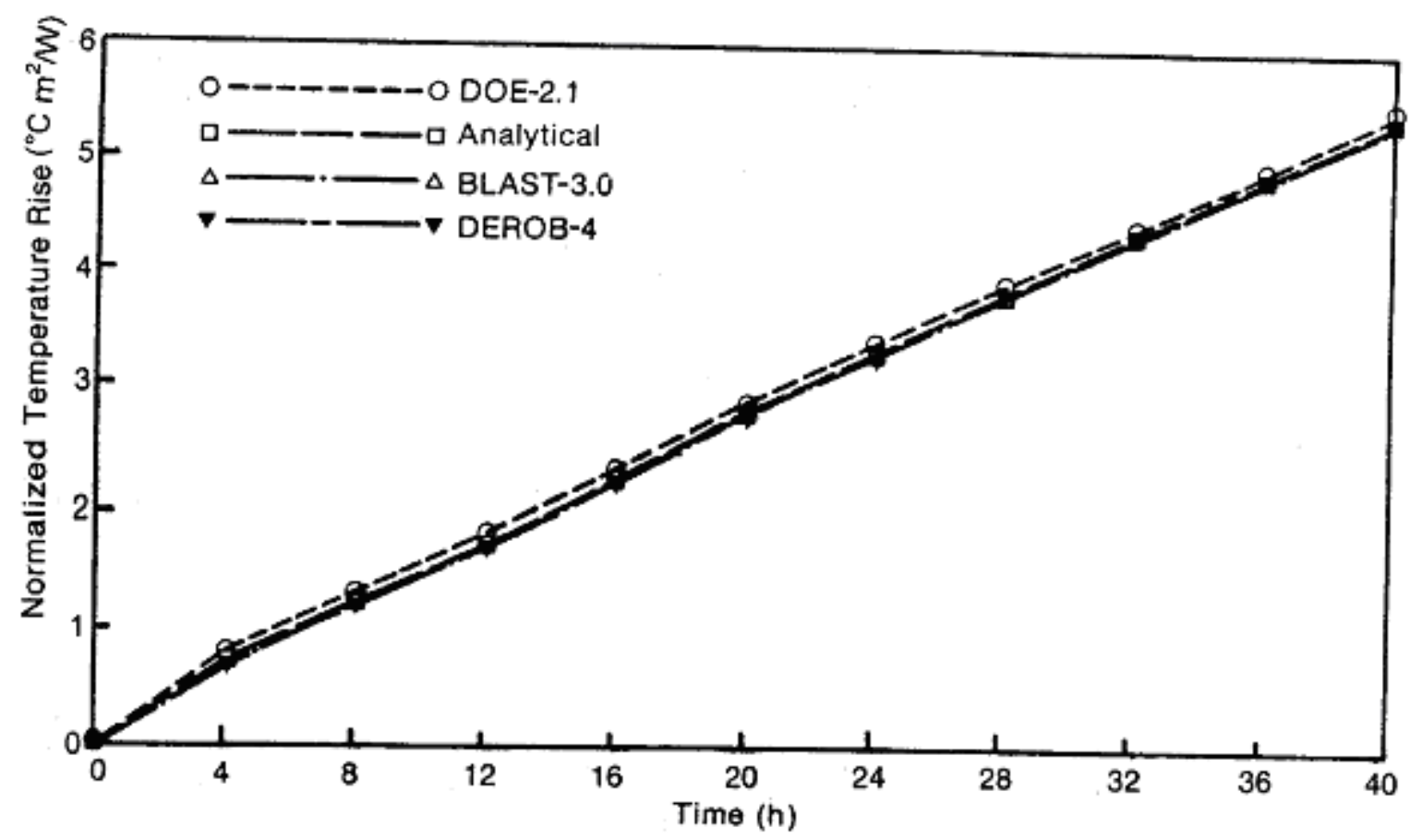

Figure 5-20. Mid-mass charge test results

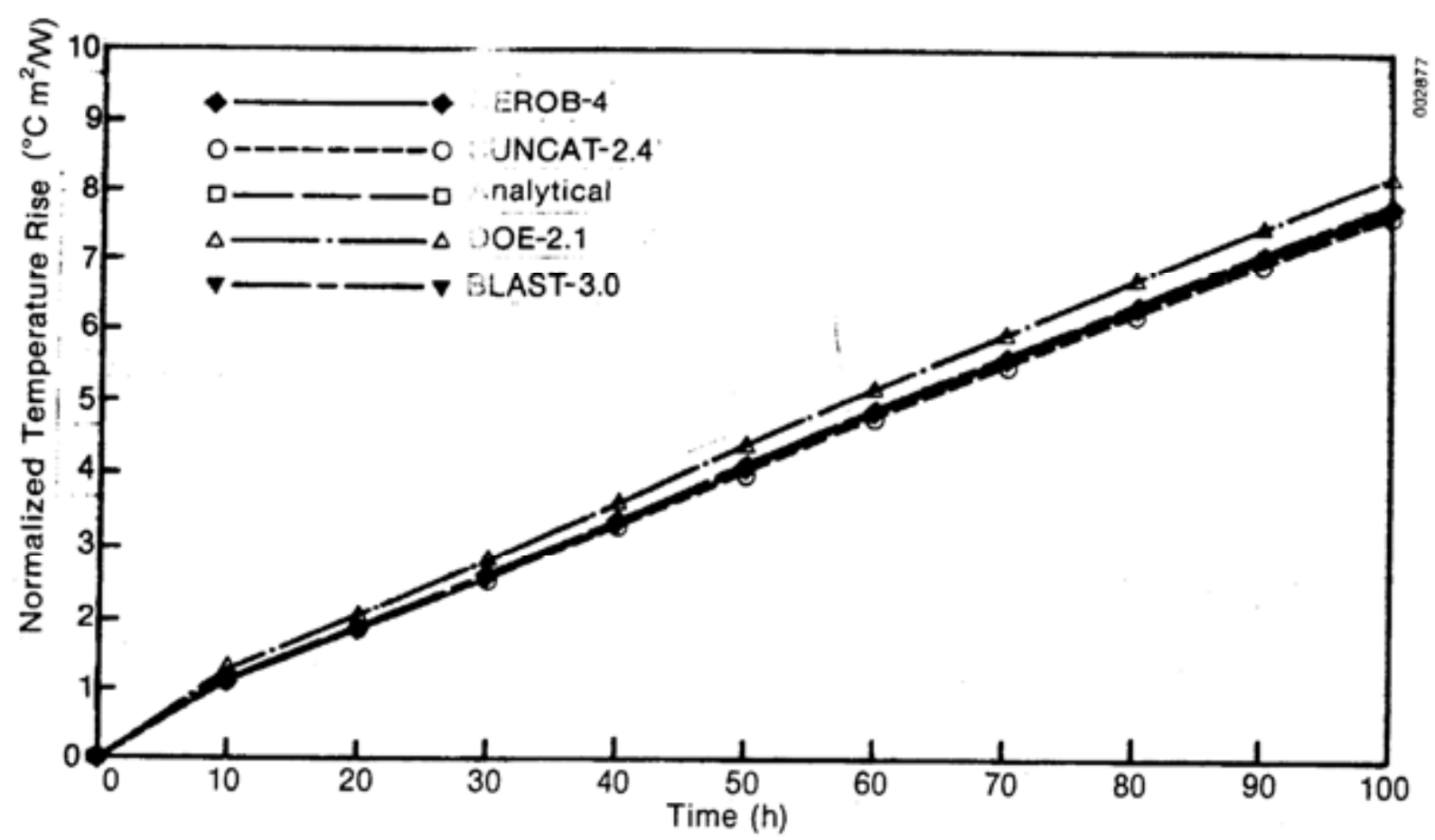

Figure 5-21. High-mass charge test results

\subsection{Conclusions}

- SUNCAT-2.4, BLAST-3.0, DOE-2.1, and DEROB-4 appear to treat the component conduction mechanism correctly, when exposed to either solar driven or convective type boundary conditions. 
- DEROB-3 was proven to be inaccurate and unusable by this method.

- The generally close agreement indicates accuracy of selected mechanisms in the codes and confirms that the analytical verification procedure is valid and has been correctly applied to the codes.

- Although originally intended as a general procedure, implementation of analytical verification proved to be highly code dependent.

- There are significant differences in window diffuse transmissivity and U-values for the different codes.

- The testing procedure proved valuable in determining assumptions in the codes concerning infiltration heat loss and undocumented heat transfer mechanisms.

\section{$5.8 \quad$ References}

1. Burch, J.D. 1980. Analytical Validation for Transfer Mechanisms in Passive Simulation Codes. Golden, CO: Solar Energy Research Institute. Unpublished report, 1980.

2. Carlslaw, H.S. and Jaeger, J.C. 1959. Conduction of Heat in Solids, 2nd ed., Great Britain: Oxford University Press.

3. Wylie, C.R. 1975. Advanced Engineering Mathematics, 4th ed., New York: McGrawHill. 


\subsection{Empirical Validation}

Empirical validation of building energy analysis programs requires taking a set of measurements that will define the problem and specify its solution. Necessary measurements include those building and meteorological parameters that become code inputs and energy fluxes and temperatures corresponding to code outputs. Instruments were installed in a two-zone test cell and a four-zone unoccupied house at the SERI field site, shown in Figure 6-1. These instruments are designed to provide data on the contributions of all important transport mechanisms, allowing calculation of zonal energy balances based on data. Experiments are planned first to provide measurement of key inputs to the codes and to validate computer simulations, emphasizing the skin loss and solar mechanisms in the test cell, as well as the infiltration and ground coupling mechanisms in the validation test house.

Section 6.1 gives a general statement of our empirical validation philosophy, including measurement practices and the methodology for code/data comparison. This section is qualitative and contains an overview of the SERI empirical validation task. Section 6.2 provides details of the implementation of this approach. Measurement procedures and the sensor installations at the SERI field site are discussed. This section provides full detail on the facility and our solutions to a number of common monitoring problems. Section 6.3 describes data acquisition. Finally, other data sources at SERI potentially useful for code validation are briefly described in Section 6.4, including four other test cells and a selective surface test box. Note that all symbols in this section are defined in Section 6.5.

\subsection{Empirical Validation Philosophy: Qualitative Presentation}

The goal of the empirical validation task is to provide data that will quantify inaccuracies in computer simulation codes that model energy transport mechanisms, concentrating on mechanisms in passive solar designs. However, gathering data is always subject to uncertainties both from measurements and from later analysis of the data. Our efforts to control these uncertainties are summarized here and described in more detail later:

- Take measurements in unoccupied controlled buildings.

- Minimize external error sources.

- Record data for individual heat transfer mechanisms and the overall building system.

- Modify building structure to eliminate inappropriate mechanisms.

- Provide consistency checks on data.

- Define methods for comparison that include effects of measurement errors.

\subsubsection{Take Measurements in Unoccupied Controlled Buildings}

The range of building types that could be used for gathering code validation data is shown in Table 6-1. We eliminated occupied buildings from our tests because of the difficulty of separating uncertainty in calculating building performance from uncertainty in occupant behavior such as opening doors and windows, using hot water, etc. A further advantage of unoccupied structures is that they can be modified experimentally. For example, tracer gases can be injected as needed, or coheating experiments run for as long as necessary. 

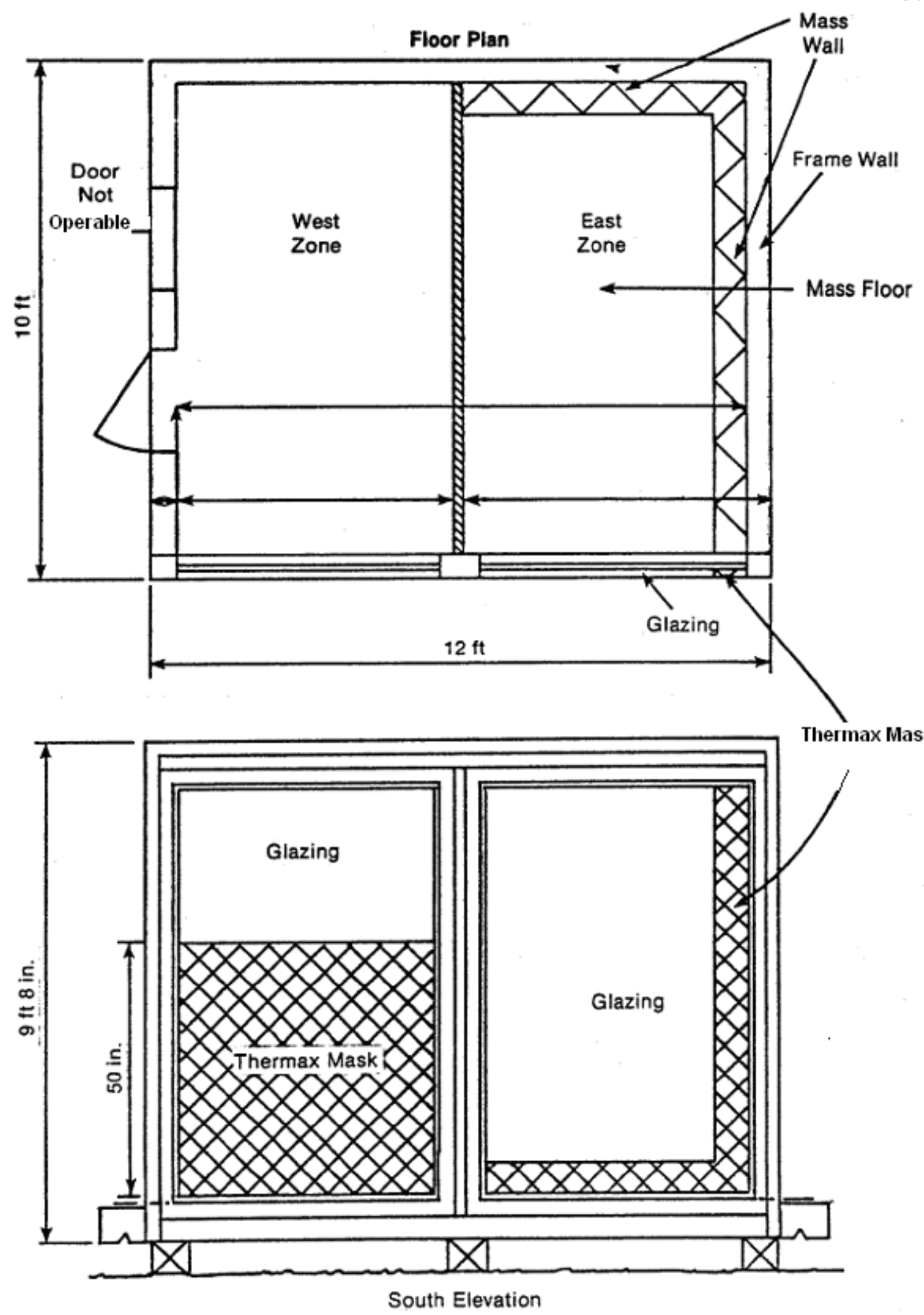

Figure 6-1A. Floor plan (a) and south elevation (b) of the two-zone test cell 


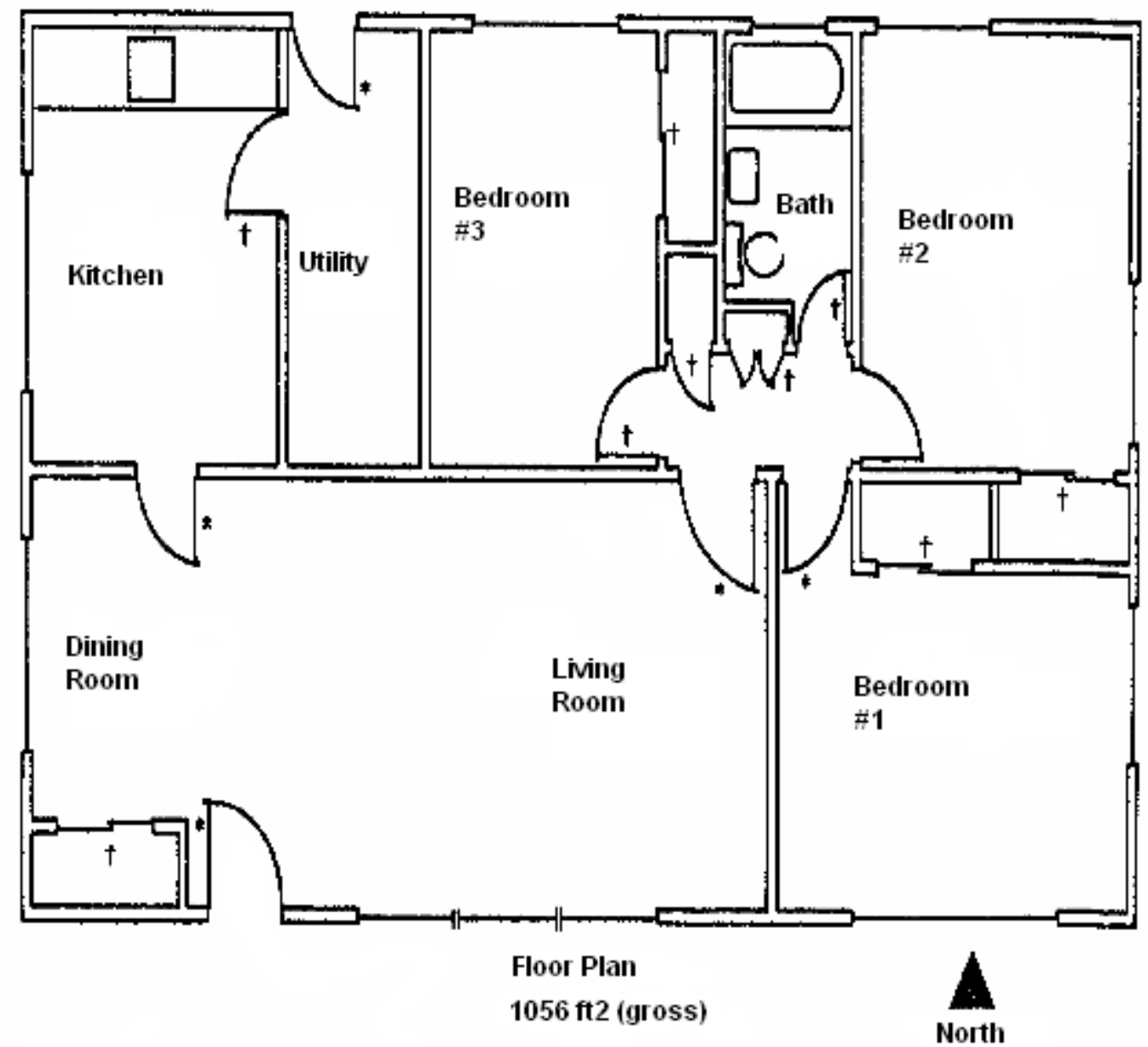

*Doors normally closed during 1981-1982 heating season.

†Doors normally open during 1981-1982 heating season.

Figure 6-1b. Validation test house 9/11/81 test site, floor plan

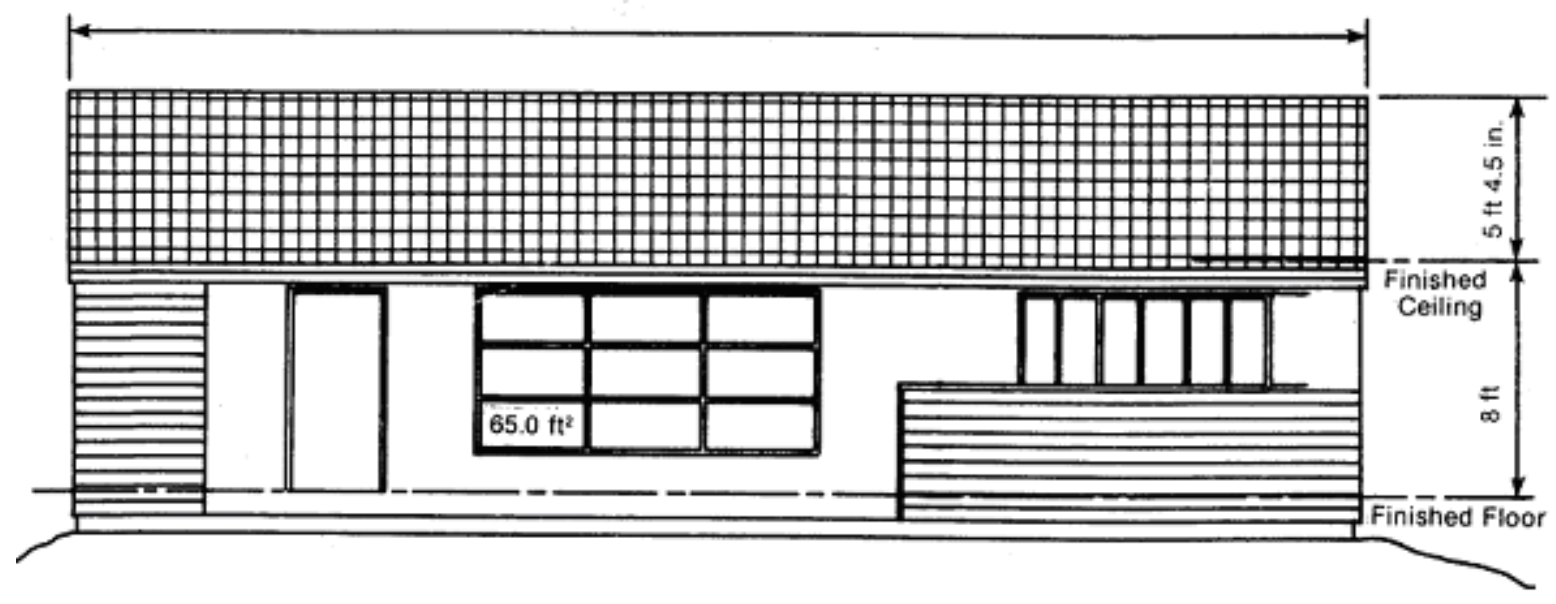

Figure 6-1c. Validation test house south elevation 
Table 6-1. Potential Validation Structures

\begin{tabular}{|c|c|c|c|}
\hline \multirow{2}{*}{ Description } & \multirow{2}{*}{$\begin{array}{l}\text { Approximate } \\
\text { Floor Area } \\
\mathrm{m}^{2}\left(\mathrm{ft}^{2}\right)\end{array}$} & \multicolumn{2}{|c|}{ Validation Assessment } \\
\hline & & Advantages & Disadvantages \\
\hline Test box & $1(10)$ & $\begin{array}{l}\text { - Mechanisms in isolation } \\
\text { - Simple to instrument and } \\
\text { control }\end{array}$ & $\begin{array}{l}\text { - No system interactions with } \\
\text { other typical components } \\
\text { - Limited applications }\end{array}$ \\
\hline Test cell & $10(100)$ & $\begin{array}{l}\text { - Total system with high } \\
\text { controllability } \\
\text { - Detailed instrumentation } \\
\text { possible }\end{array}$ & $\begin{array}{l}\text { - Not typical size for some } \\
\text { mechanisms } \\
\text { - Not all mechanisms present }\end{array}$ \\
\hline $\begin{array}{l}\text { Unoccupied } \\
\text { house }\end{array}$ & $100(1000)$ & $\begin{array}{l}\text { - De facto typical for residential } \\
\text { natural mechanisms } \\
\text { - No occupant uncertainty }\end{array}$ & $\begin{array}{l}\text { - No occupant-influenced } \\
\text { mechanisms are present }\end{array}$ \\
\hline $\begin{array}{l}\text { Occupied } \\
\text { house }\end{array}$ & $100(1000)$ & $\begin{array}{l}\text { - Many important occupant- } \\
\text { related mechanisms to study }\end{array}$ & $\begin{array}{l}\text { - Some occupancy } \\
\text { uncertainty } \\
\text { - Hard to manipulate, control, } \\
\text { or instrument }\end{array}$ \\
\hline Commercial & $>200(2000)$ & $\begin{array}{l}\text { - Many zones } \\
\text { - Internal gains domination }\end{array}$ & $\begin{array}{l}\text { - Occupancy uncertainty } \\
\text { - Very hard to control or } \\
\text { instrument }\end{array}$ \\
\hline
\end{tabular}

As Table 6-1 shows, unoccupied structures offer a wide range of possible sizes and validation features. Data from a test box reflect the energy performance of components that are highly instrumented and controlled but cannot provide information on interactions between components within a building. A test cell has all the features of a building (e.g., windows, walls, and zones). Also, it is a simple configuration that can be easily instrumented and manipulated. However, a test cell is too small to permit the study of many scale-dependent mechanisms such as multidimensional conduction and advection. A test house approximates existing buildings and is the only suitable tool for studying these mechanisms. However, analyzing the zonal energy balances will be more difficult than analyzing a test cell because of the numerous complex energy paths in residential-sized buildings. Therefore, all these approaches can be fruitful and are used at the SERI field site. Most of the effort is focused on the test cell and the test house shown in Figure 6-1.

\subsubsection{Minimize External Error Sources}

The complexity of building energy simulations implies errors or discrepancies in code-data comparison that must be defined and assessed. For purposes of our empirical validation work, the most important error sources are (see Section 3.0): (1) energy mechanism modeling inherent in the codes, (2) numerical encoding, (3) building parameters and schedules, and (4) environmental data. These simulation components are shown in Figure 6-2 [1]. The first two are called internal error sources, and the latter two, which are input data for the numerical model, are called external error sources. Empirical validation involves all four discrepancy sources simultaneously, so discrepancies between code and data may not be caused by the simulation alone. For example, if a wall conductivity estimate is uncertain by $50 \%$, significant code-data deviation can be expected and cannot be attributed to an internal code problem. 


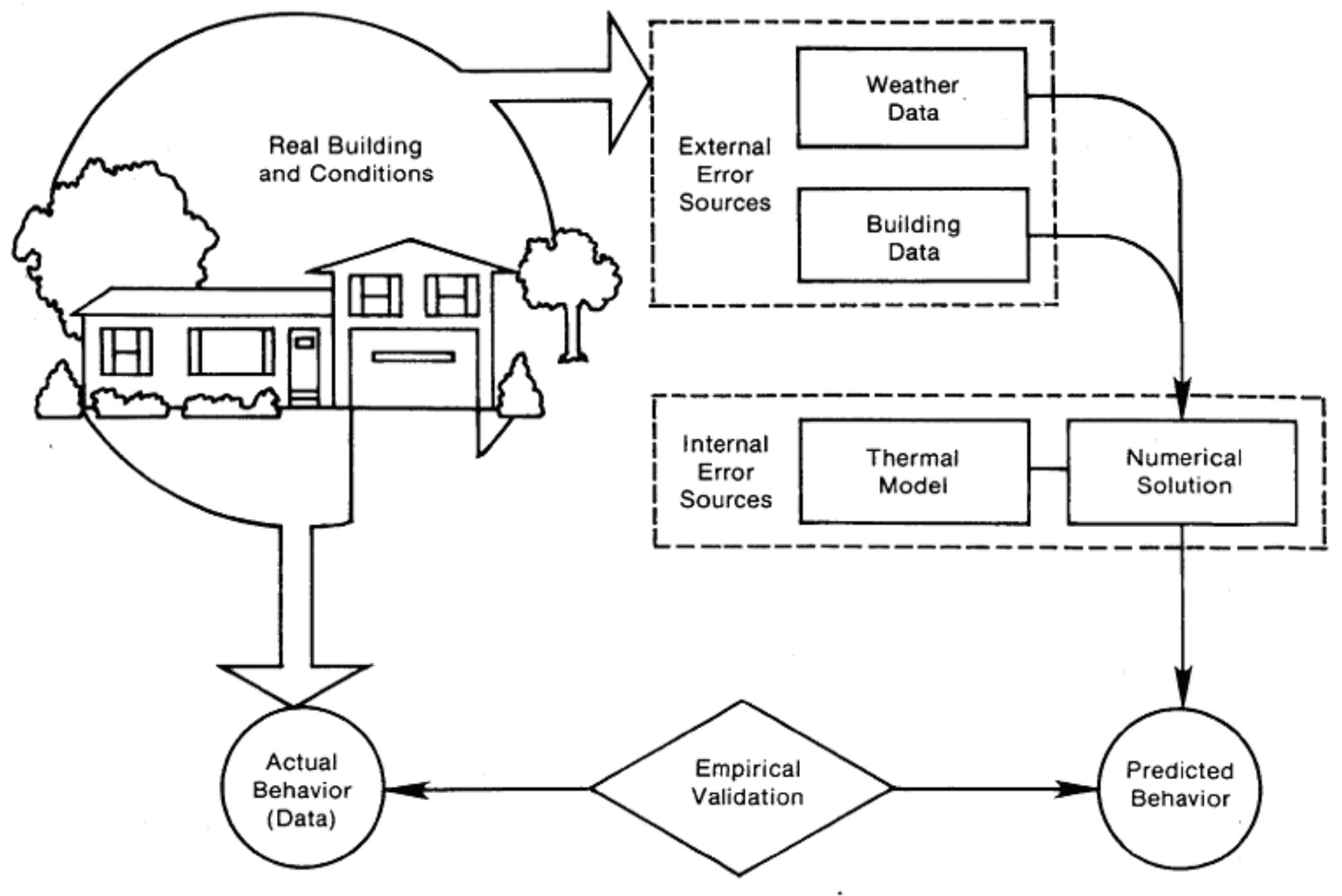

Figure 6-2. Simulation components 
We intend to assess the accuracy of internal components: the thermal model and its numerical encoding. Hence, the empirical effort must minimize external error sources, which are the building and environment inputs. Therefore, measurements and experiments must be done carefully to obtain accurate values for these inputs. Errors that remain will be propagated onto the comparison variables to define the comparison interval, as in Section 6.1.6. We will assess the question of user errors or uncertainties in translating building plans into input parameters using handbook values or estimates of thermophysical parameters. This question is being pursued elsewhere, in the case of commercial buildings [2].

\subsubsection{Record Data for Individual Heat Transfer Mechanisms and the Overall Building}

To validate the key thermodynamic calculations available from the codes, two different types of data are needed. First, data must be taken to define the overall building energy performance. This overall system level includes zone air and global temperature data and (if temperature controlled) auxiliary energy measurements. These data summarize building performance and provide the information most designers want. Since the system level analysis contains the effects of many operative mechanisms, interpretation of discrepancies and detection of canceling errors is difficult. Most past empirical validation has been restricted to this level (see Section 2.0), because it is the simplest to instrument and provides bottom-line information.

However, data should also be taken to isolate those transport mechanisms that significantly affect building energy performance. Important mechanisms are summarized in Table 6-2, grouped as conduction, convection, and radiation. As in Figure 6-2, the codes must first choose explicit deterministic models for all mechanisms to be included (thermal model) and then solve the resulting equations simultaneously (numerical model). The approaches in the codes studied here are discussed in Section 7.0 of this report.

Table 6-2. Passive Energy Transport Mechanisms

\begin{tabular}{|l|l|}
\hline Conduction: Measure temperatures and & Structural elements \\
conduction fluxes & - Skin and interzonal opaque walls \\
& - Glazings \\
& - Ground coupling \\
\hline \multirow{5}{*}{ Convection: Tracer gas, special experiments } & Film coefficients \\
& - Inside surfaces-free and forced convection \\
& - Outside surfaces-forced convention \\
& - Infiltration \\
& - Zone to zone \\
& - Natural convection through doorways \\
& - Natural convection through cracks \\
& - Stratification \\
\hline \multirow{5}{*}{ Radiation: Measure radiant fluxes } & Infrared surface coupling \\
& - Internal surfaces \\
& - External surfaces (sky temperature) \\
& Solar \\
& - External absorption \\
& - Glazing transmission and absorption \\
& - Internal absorption \\
\hline
\end{tabular}


The two-level approach will allow the identification of those mechanism inaccuracies that lead to system level inaccuracies. For example, all the codes simulate both infiltration and conduction through lightweight skin elements. These mechanisms will act similarly and cannot be separated at the system level. Only by taking separate measurements of each mechanism can one determine whether the infiltration or the skin conduction algorithm is contributing to any errors observed on the system level that shows steady-state coupling characteristics.

Heat transfer mechanisms can be isolated by direct measurements. In addition, their effect on the system level variable can be displayed by sequencing the building configuration and by planning experiments to introduce or substantially change one mechanism at a time. Components of the SERI structures that can be varied or reconfigured are listed in Table 6-3. The principles of experiment planning are: (1) proceed sequentially from simple to more complex configurations, providing incremental addition of mechanisms between experiments; and (2) at a given configuration, systematically vary parameter values to enhance that parameter's effects.

Table 6-3. Structural Variables of the Test Cell and Validation Test House

\begin{tabular}{|c|c|}
\hline \multirow{4}{*}{ Test cell } & $\begin{array}{l}\text { Cell glazings } \\
\text { - Unshaded, shaded, or completely blocked } \\
\text { - Variable area, with thermax masks }\end{array}$ \\
\hline & $\begin{array}{l}\text { Zone constraint } \\
\text { None, or } \mathrm{T}_{\text {zone }} \geq \mathrm{T}_{\text {set }}{ }^{(\mathrm{t})} \text { (heating) or } \mathrm{T}_{\text {zone }} \leq \mathrm{T}_{\text {set }} \text { (cooling) in either or both zones }\end{array}$ \\
\hline & Zone divider wall R-value \\
\hline & $\begin{array}{l}\text { Night ventilation } \\
\text { Up to } 15 \mathrm{ACH} \text {, various on/off strategies }\end{array}$ \\
\hline \multirow{4}{*}{$\begin{array}{l}\text { House (Retrofit } \\
\text { measures to be } \\
\text { applied) }\end{array}$} & $\begin{array}{l}\text { Conservation retrofit } \\
\text { - Insulation level of walls and ceilings } \\
\text { - Foundation wall and crawl space insulation } \\
\text { - Storm windows } \\
\text { - House tightness } \\
\text { - Mass floor }\end{array}$ \\
\hline & $\begin{array}{l}\text { Zone coupling } \\
\text { - Doors sealed between zones } \\
\text { - Open interzonal doors }\end{array}$ \\
\hline & Passive solar addition \\
\hline & Stratification/fans \\
\hline
\end{tabular}

\subsubsection{Modify Structure to Eliminate Inappropriate Mechanisms}

We know that the codes cannot accurately simulate all building components, so for our experiments we chose to selectively modify either the structure or its operation to minimize troublesome mechanisms that cannot be adequately measured or modeled. In this way, the remaining mechanisms can be studied in a simpler context where interpretations of results are more meaningful.

\subsubsection{Test Cell Case}

For the test cell shown in Figure 6-1 we eliminated or minimized all mechanisms considered to be uncertain or impossible to simulate. These mechanisms include natural convection (interzonal convection, stratification, and infiltration), ground coupling, heat transfer across opaque wall air gaps, and nearby building solar reflections. Thus, we retain or make dominant those mechanisms 
(mainly wall conduction and solar absorption) that the codes are designed to analyze. The most important features of direct gain and sunspace solar buildings remain in the analysis. In particular, mass and frame wall conductions are treated in detail, interzonal conduction coupling is emphasized, and the solar driving force is strong.

\subsubsection{Va1idation Test House Case}

As in the cell case, stratification and interzonal natural convection are eliminated for this study; we will include them in future work. There are two important differences between the cell and the house. First, infiltration is an important piece of the house energy budget and must be measured directly. Second, ground coupling through the unvented crawl space is also present and must be defined. Wall conduction and solar mechanisms are also present but will not be studied in the same detail possible in the test cell. Night ventilation was included during the summer cooling runs in 1982. Test house configurations are to be of the temperature-constrained and free-floating types, with emphasis on the former as most representative of realistic circumstances.

\subsubsection{Provide Consistency Checks on Data}

Since energy transfers follow the first law of thermodynamics, we should be able to write an energy balance equation for relevant systems and determine the accuracy of our data. An imbalance in the equation $\mathrm{Q}_{\mathrm{IN}}=\mathrm{Q}_{\mathrm{OUT}}+\delta \mathrm{Q}_{\mathrm{STORED}}$ is caused by misleading data, incorrect analysis, or mechanisms not represented in the measurements. A similar procedure is used for aspects of solar data and for film coefficients measurements. These procedures are detailed in Section 6.2.

We intend to treat all sensors skeptically and check them with documentable calibration. Pyranometers are to be calibrated at the National Solar Radiation Laboratory independently from the manufacturer. Known temperature baths and voltage are always online for temperature and voltage checks, heat flux transducers and wattmeters were independently checked, and NBS traceable voltage calibrations were performed on all voltage digitizers.

\subsubsection{Defined Methods for Comparison Including Effects of Measurement Errors}

We intend to compare measurements to the calculated outputs of public domain building energy analysis codes discussed in Section 7.0. We can easily define comparison measures, as shown in Table 6-4. These comparison measures are standard statistical quantities calculated as the difference between computed and measured values. However, to interpret or draw conclusions about code accuracy from these measures requires that we define an appropriate comparison interval for each variable being compared between code and data. Two components will affect the comparison interval: direct measurement errors and indirect input errors. Direct measurement errors are defined as the measurement standard deviation, and initial estimates are listed in Table 6-5. However, all code-computed variables also depend implicitly on the input values of the descriptive building and environment parameters. For example, errors in calculating irradiance on external surfaces are affected by errors in the beam and global solar radiation inputs, regardless of the accuracy of the radiation processing model in the code. Likewise, the zone temperature in a free-floating system will be affected to some degree by uncertainties in almost all input parameters. In general, as the code inputs are allowed to vary within an appropriate measurement interval, the computed values are expected to vary. These variations define limits 
within which the code can reasonably be expected to agree with data, since the measured data may fall anywhere in the comparison interval.

Table 6-4. Comparison between Code and Data

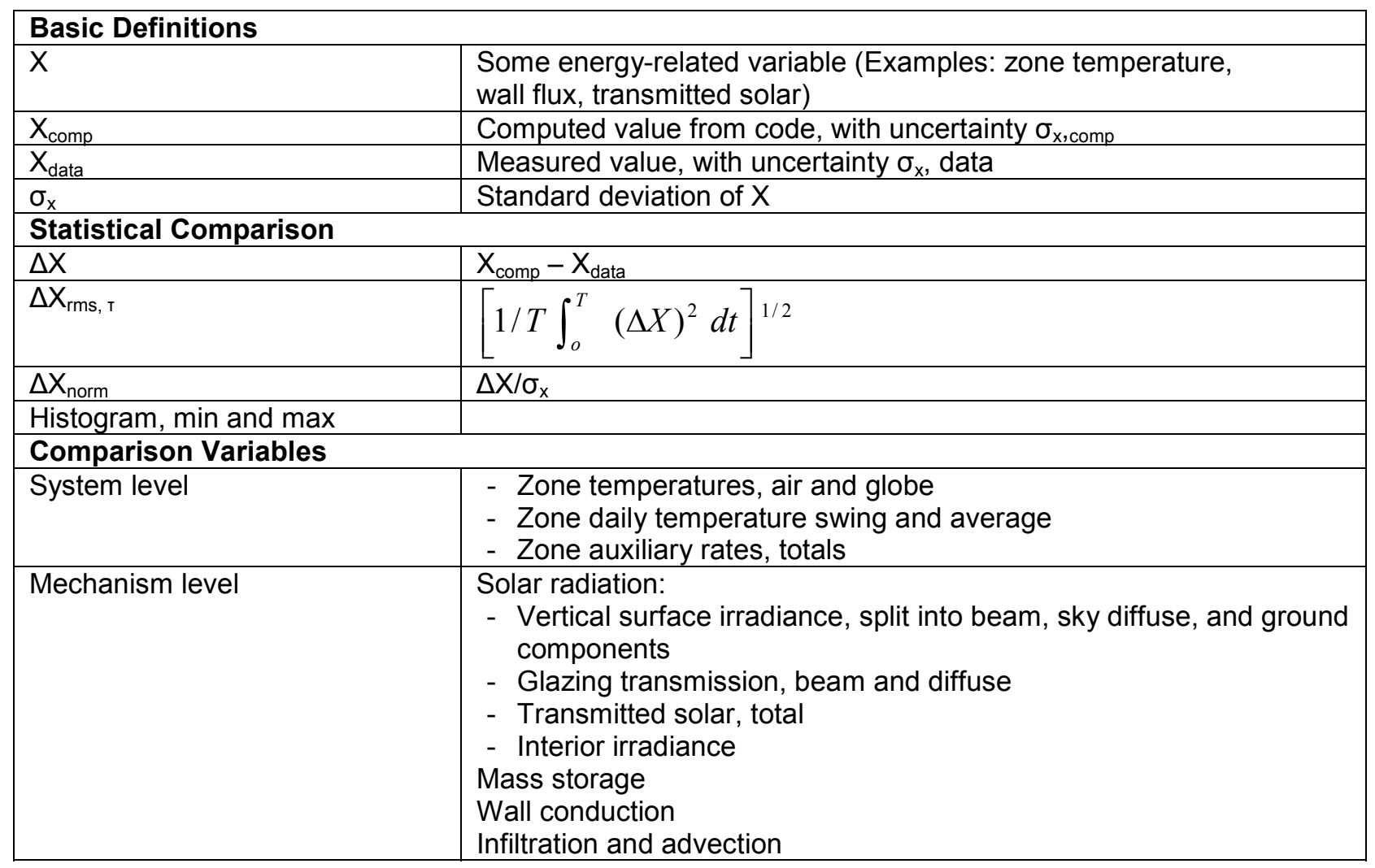

Table 6-5. Measurement Errors

\begin{tabular}{|c|c|}
\hline \multicolumn{2}{|l|}{ Direct Measurable } \\
\hline Temperature T & $1.0^{\circ} \mathrm{F}\left(0.6^{\circ} \mathrm{C}\right)$ \\
\hline Temperature differentials $\Delta \mathrm{T}$ & $0.05 \Delta \mathrm{T}$ \\
\hline Surface conduction fluxes $\dot{Q}_{\text {cond }}$ & $0.10 \dot{Q}_{\text {cond }}$ \\
\hline Surface solar irradiance & $\begin{array}{l}0.02 \mathrm{I} \text {, total and beam } \\
0.04 \mathrm{I}_{\text {diff, }} \text { diffuse only }\end{array}$ \\
\hline Sky radiance $R(\theta, \varphi)$ & $\pm 0.1 \mathrm{R}(\theta, \Phi)$ \\
\hline Auxiliary power $\dot{Q}_{\text {heater }}$ & $0.01 \dot{Q}_{\text {heater }}$ \\
\hline Internal gains $\dot{Q}_{\text {internal }}$ & $0.01 \dot{Q}_{\text {internal }}$ \\
\hline \multicolumn{2}{|l|}{ Building Inputs } \\
\hline Wall UA & $\sim(0.10)$ UA \\
\hline Infiltration & $\sim 0.3 \dot{Q}_{\text {inf }}\left(0.1 \dot{Q}_{\text {inf }}\right.$ with monitoring equipment $)$ \\
\hline Optical Properties: surface absorptivity & $\begin{array}{l}\alpha_{s}=\text { solar absorptivity: } \sigma_{, \alpha s}= \pm(0.02+0.02 \alpha) \text {, to be added in } \\
\quad \text { quadrature with sample-to-sample errors } \\
\alpha_{1}=\text { infrared absorptivity: } \sigma_{\alpha \mid}= \pm 0.05\end{array}$ \\
\hline Specific heat $c_{p}$ & $\pm 1-10 \%$, depending on source of data \\
\hline Glazing index, extinction coefficient $\eta, \mathrm{K}$ & Typically, handbook/manufacturers' data; errors vary \\
\hline
\end{tabular}


Comparison intervals are to be set by propagating all of the input building and environment parameter errors on to the variable in question, using the codes themselves for this application. In this process, single input parameters are to be varied within error limits, and the changes to the output parameters are noted. Changes due to all relevant input parameters are added in quadrature, along with the direct measurement error, to finally define the input-dependent comparison interval. Note that this approach ignores parameter interactions, and a Monte Carlo approach may be needed to define the input-dependent comparison intervals more precisely.

Once the direct and indirect comparison error intervals are determined, the comparison measures can all be normalized as in the Table 6-4 definitions, dividing each measure by the comparison interval. These normalized measures clearly indicate how the code and data discrepancies relate to error impact. For example, normalized values well below 1.0 indicate that the code tracked within error, whereas values well above 1.0 indicate that the code was significantly outside the comparison interval. Hence, for each measure in Table 6-4, we will compute the physical unit and normalized measures. We do not consider here what degree of discrepancy should be considered tolerable for the sake of design estimates, given all problem uncertainty.

\subsection{Implementation of Validation Philosophy: Technical Presentation}

This section describes our field site measurements and installations. First, general sensor practices and measurement problems are discussed. Second, an overview of the cell and house is given, including particular aspects of their instrumentation and structural modifications made for minimizing intractable mechanisms. Third, the measurements that are required to precisely define the descriptive code inputs are presented. Fourth, data being acquired to validate code output calculations are discussed with emphasis on code treatment of solar radiation. Last, the experiment plans for the structures are given, indicating the usefulness of a sequence of experiments in which the effects of mechanisms are isolated by being selectively introduced or modified.

\subsubsection{Measurement Methods}

\subsubsection{General Description of Sensors}

We installed sensors for measuring temperatures, thermal fluxes, auxiliary power, solar irradiances, sky temperature, infiltration rates, and most standard meteorologic parameters. The problems encountered in using these devices are always faced by those instrumenting Class A sites. Hence, it is useful to describe the problems and our solutions to some of them.

Temperature Sensors. Sensors use type J thermocouples (iron-constantan). We used 0.5\% wire, and each thermocouple installed was checked for accuracy at the ice point. RMS deviation was about $0.2^{\circ} \mathrm{F}\left(0.1^{\circ} \mathrm{C}\right)$. An absolute accuracy of about $1.0^{\circ} \mathrm{F}\left(0.6^{\circ} \mathrm{C}\right)$ can be expected for field operation, as shown in Table 6-5. Surface temperature data required some care. For the final inch of the wire, the thermocouples were set into a linear indentation that held the wire snugly with the couple weld joint located as close to the surface as obtainable, 0.13 in. $(\sim 3 \mathrm{~mm})$ deep. The indentation was then filled with epoxy and the surface smoothed over. The installation was given solar/infrared properties similar to the wall being analyzed, painting the area with paint identical in solar absorptivity to that used on the wall. Thermocouples on the glazing surface were specially treated, since solar radiation absorbed by the thermocouple raises the temperature beyond the true surface value. Hence, for the glazings, the thermocouple was first covered with 
about 0.75 to 1 in. (19 to $25 \mathrm{~mm}$ ) of reflective aluminum foil tightly wound around the thermocouple. With the end pressed against the glazing, we applied a narrow line of clear epoxy about 0.25 to $0.5 \mathrm{in}$. (6 to $13 \mathrm{~mm}$ ) long to the thermocouple to secure it to the glazing surface. Despite these precautions, glazing surface temperature data must be treated with skepticism until any necessary correction is determined. This will be done by comparing sunlit thermocouple values to those from an infrared temperature sensor.

Air temperatures were measured with sensors protected by radiation shields. Internal shields not in sunlit spaces consisted of two concentric, deoxidized aluminum cylinders $\sim 4$ in. $(\sim 10 \mathrm{~cm})$ long with outer shield $\sim 1.5$ in. $(\sim 4 \mathrm{~cm})$ in diameter. Any sensors that could be irradiated by direct beam radiation were additionally protected by a third outer shield of highly reflective double aluminized Mylar with $\alpha_{\mathrm{s}} \sim \varepsilon_{\mathrm{IR}} \sim 0.05$. External shields were larger $(\sim 1 \mathrm{ft}[\sim 0.3 \mathrm{~m}]$ long, with a 4in. $[10-\mathrm{cm}]$ diameter external tube) and were covered, to prevent solar/moisture perturbation and to maintain good flow. The triply layered shields showed no sensitivity to direct beam irradiance, whereas the two double-layered shields were found to be in error $5^{\circ}$ to $8^{\circ} \mathrm{F}\left(2^{\circ}\right.$ to $\left.3^{\circ} \mathrm{C}\right)$ under beam conditions. Zone globe temperature is measured by a thermocouple inside a blackened copper toilet bowl float mounted at a height of $5.3 \mathrm{ft}(1.6 \mathrm{~m})$.

Fluxmeters. We measured thermal fluxes directly for all typical wall elements, so that wall properties could be deduced in situ, flux correlated with wall temperatures, and energy balances in a zone performed with a minimum of assumptions. Thermal fluxes in opaque walls were measured directly using thermopile fluxmeters from Valley R\&D Lab, Model T225. In general, these transducers are installed near the interior surface, but buried to operate in the conductiononly mode in which they are calibrated [3]. The surface was etched out to 0.13 to 0.25 in. (3 to 6 $\mathrm{mm}$ ) depth, the sensor installed and covered with Patch-kote $\AA$, and the surface smoothed and repainted. Electrical leads were insulated with Glyptol ${ }^{\circledR}$. The thermal mass over the sensor smoothed out the transients from small-time film fluctuation and gave flux at a location similar to the idealized surface to which code outputs for surface flux apply. Note that additional errors must be considered if the fluxmeter is buried in a medium whose conductivity is large compared to that of the fluxmeter. The fluxmeters agreed within $4 \%$ with careful rechecking of conduction mode calibrations. Relative agreement before recalibration, however, was $\sim 8 \%$. Absolute accuracy for the conduction calibration factor was $\sim 5 \%$ [3] and will be independently checked when the SERI standards lab completes its flux calibration installation (FY 1984). Hence, the fluxmeters are accurate to about $10 \%$, combining errors in a quadrature.

Attaching fluxmeters directly to a surface can lead to spurious readings for several reasons. First, an additional local resistance, including contact resistance and meter resistance, is introduced, causing a reduction in the measured value versus the unperturbed value. Second, surface properties, including texture and protrusion (affecting the convective coupling) and surface emissivity (affecting radiant coupling) may change. Hence, any surface-mounted fluxmeter readings must be treated with caution. The problem is unavoidable and most serious for glazings where the fluxmeter cannot be buried to operate in the conduction mode only as described earlier. Thus, we intend to apply a calibration factor obtained when the meters are surface mounted on glazing assemblies as opposed to manufacturers' values, which are obtained in a conduction-only mode. We will determine this difference, which can be $20 \%$ or larger, by using a guarded hot box apparatus designed and constructed at SERI. 
Fluxmeters mounted on glazings cause additional problems because solar absorptions induced by the fluxmeter cause spurious fluxes. Several measures were taken to minimize the problem. First, the fluxmeter surfaces were modified. The surface contacting the glass and facing outward was covered with reflective foil. The inward facing surface was painted white to minimize absorption of back-reflected light while keeping the infrared emissivity near 1.0. Second, to keep good contact between glass and transducer without introducing an absorptive layer, either sodium silicate (having optical transmission similar to glass) or transparent double-sticky tape was used as a surface adherent.

Daytime fluxmeter readings must be treated with great caution in any case and probably cannot be used without correction for solar interaction with the fluxmeter. As shown in Figure 6-3, the fluxmeter on the glazing surface induces two perturbations. First, a flux is absorbed where the fluxmeter and the glazing surface interface. Second, extra absorption is induced in the glazing pane next to the fluxmeter as a result of light reflected from the fluxmeter. Assuming a onedimensional flow, a fraction (about 0.5 for double pane) of this spurious absorbed solar energy is measured by the fluxmeter and passes into the room. The other portion of the spurious flux is lost to the outside (single pane) or glazing air gap (double pane) and can be ignored. Thus, the true glazing thermal flux is:

$$
\dot{Q}_{\text {glaz }}=\dot{Q}_{\text {meas }}-f\left[\alpha_{f m}+\left(1-\alpha_{f m}\right) \exp -\kappa L_{\text {eff }}\right] G_{\text {vert }}^{\text {tran }}
$$

Using fluxmeters with three different measured values for $\alpha$, the above theory can be tested for accuracy and consistency. The quantity f will be adjusted to fit the results so that $\dot{Q}_{\text {glaz }}$ as corrected is the same for each $\alpha$.

Fluxmeters in contact with the ground surface were installed in the house crawl space. These fluxmeters were carefully sealed against moisture penetration. We used a meter with a fiberglass covering impregnated with epoxy to inhibit moisture-related spurious voltages.

Even though a buried ground fluxmeter may perfectly indicate local flux, the installation procedure may perturb the local flux. The ground resistance can be changed, for example, by breaking up and introducing voids into packed soil or by changing the moisture content of soil above the meter. Hence, the best installation procedure disturbs the soil near the meter the least. At our site, with cohesive small-grained surface soils, that goal can be realized by first digging a shallow trench next to the fluxmeter location and inserting the fluxmeters into a thin gap created by a sawblade and a simple jig sized to just fit the fluxmeter body. The gap is backfilled, and the trench tamped with the soil originally removed from the trench.

Auxiliary Power. Hall effect watt meters from Ohio Semitronics, Inc. (PC5 Series) were used to monitor power to electric heaters, destratifiers, and other internal equipment. These transducers include voltage/current phase effects in motors (power factor) automatically and are accurate to $0.5 \%$. Manufacturers' calibration will be checked to $\sim 0.1 \%$ at the standards lab at SERI. If only device currents were measured, voltage fluctuations would not be considered, and the resulting power values in significant error. In the house, the total input was additionally monitored, so the sum of the individual measurements can be cross checked against the total power data. 


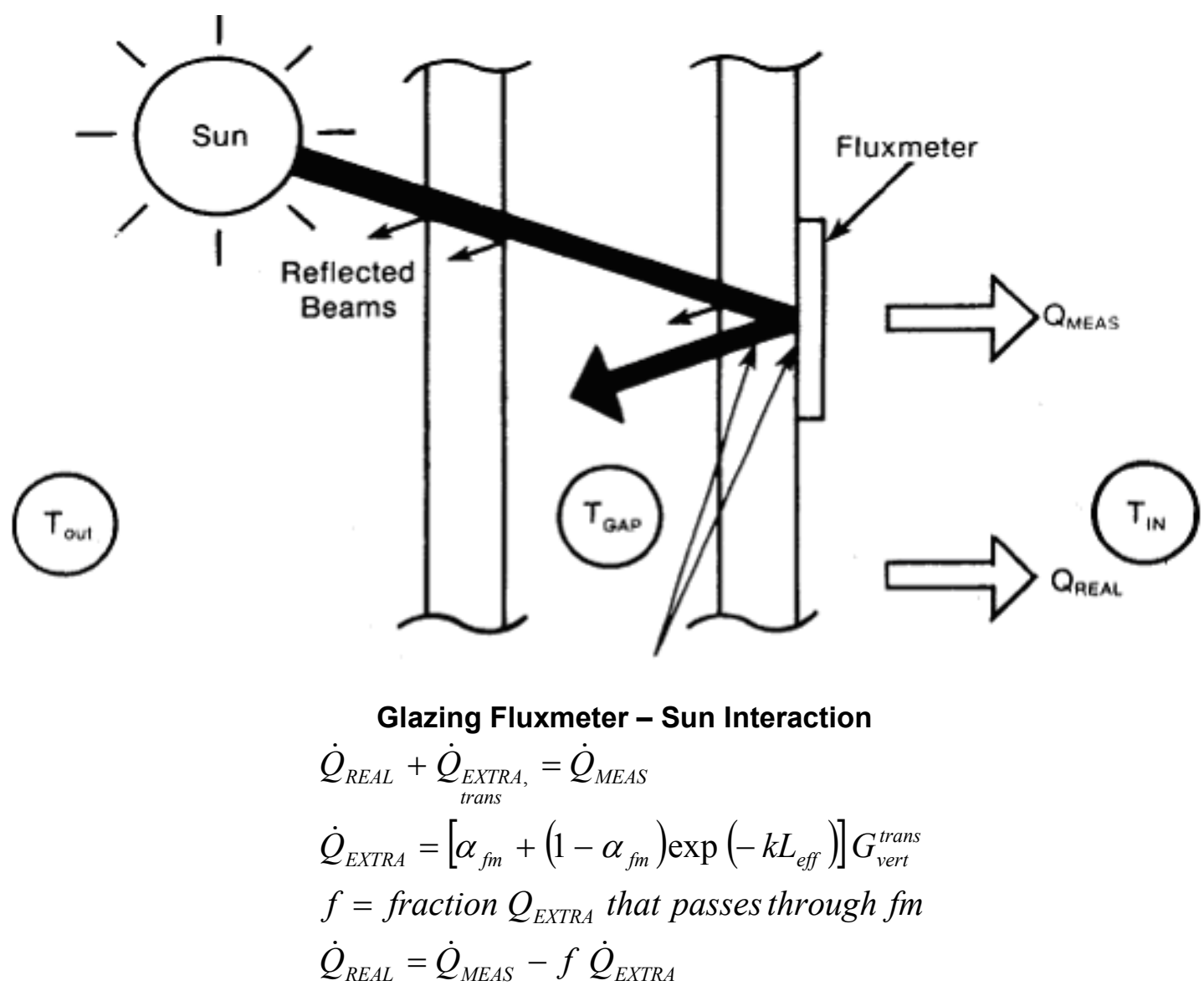

Figure 6-3. Daytime glazing conduction

Pyranometers. Thermopile sensors were employed, including Eppley Model PSP, Kipp and Zonen CM5/6, and HY-CAL Model P-8405-A. Although less expensive, spectrally dependent photovoltaic sensors would introduce $\pm 20 \%$ uncertainty in diffuse solar fluxes, without careful floating calibration [4]. In any case, these sensors should not be used to monitor radiation reflected from interior surfaces. Each sensor is to be sent to the National Solar Radiation Facility at the National Oceanic and Atmospheric Administration (NOAA) in Boulder, Colorado, for independent calibration. Manufacturers' values were generally within several percentage points of the NOAA calibration; however, several calibration changes of nearly $10 \%$ were required, consistent with other results on radiation calibration [5]. These sensors were used specifically for data on the cells and house. Measurements of global horizontal, vertical, and latitude tilt south sky, and direct beam data are provided by others at the field site [6], with independent calibrations maintained via a Davas-type cavity pyranometer.

Radiation shields. We need to measure the contributions from the south sky and the south foreground separately. To measure these quantities directly, a south vertical pyranometer will be outfitted with an artificial horizon that blocks either the ground or sky. The design chosen is shown in Figure 6-4 for the sky measurement case. Part of the sky is blocked for the lower half of the detector, and part of the ground is seen for the upper half of the detector. To some extent, these two errors will cancel, as calculated in Figure 6-4. 
The view factor between detector and ground can be calculated by direct integration from the basic differential area expressions, using the simplifying assumptions listed Figure 6-4. For bright ground ( $\rho 0.8$, as for fresh snow) and for very clear sky (with the diffuse fraction on horizontal as 0.15$)$, then a radius of about $32 \mathrm{~cm}(13 \mathrm{in}$.) gives about a $2 \%$ error in the measurement. These numbers represent an extreme case, and the error will be acceptable for any radius beyond a foot or so for a detector radius of $13 \mathrm{~cm}$ (0.5 in.). An Eppley Model PSP [6] will be outfitted with a horizon shield, to provide continuous monitoring of radiation incident on south vertical surface emanating only from the sky.

An alternative design could be considered, reducing the pyranometer surface to be entirely below the level of the shielding ring. In this case, no area of the detector senses ground, and no assumptions about ground reflectivity are needed. However, detailed calculation shows that the view factor to the sky is correspondingly reduced so, for the isotropic sky assumption, the sky diffuse irradiance will have more than a $5 \%$ error for a $30 \mathrm{~cm}$ (12 in.) shielding radius. Although a correction would improve measurement accuracy, it would be difficult to weight the correction, because of the effect of horizon brightening.

The same analysis will apply to inverting the artificial horizon shield to block the sky so only ground-reflected diffuse radiation is present. A Zonen CM6 pyranometer in front of the test cell will be shielded occasionally to sense ground radiation.

Infrared sky temperature. We measured night sky radiant flux directly with an infrared net radiometer, Geotech Model 188, with a temperature-compensated thermopile that measures the $\Delta \mathrm{T}$ between a silvered lower horizontal surface and a black upper horizontal surface. The surfaces are aspirated to minimize film coefficient fluctuations from the wind. The output voltage is directly related to the infrared flux from the black surface to the sky only when no solar radiation is incident (i.e., at night). An attempt will be made to correct daytime readings using the methods in Cooper et al [7]. However, this procedure may not be reliable, since the upper surface is black for both solar and infrared bands, making the correction large and correspondingly less reliable. Sky temperature can be inferred to be $\mathrm{T}_{\mathrm{sky}}=\left(\mathrm{T}_{\mathrm{amb}}-\dot{Q}_{\mathrm{ir}} / \sigma\right)^{1 / 4}$.

Tracer gas concentration. Tracer gas decay methods, discussed more fully in Section 6.2.3.1, were implemented on the house and the cell. The tracer gas concentration was measured with a Foxboro, Inc., Miran Model 100 infrared analyzer for detecting sulfur hexafluoride $\left(\mathrm{SF}_{6}\right)$ tracer gas [8]. The instrument exhibits undocumented but severe temperature-related zero drifts, as much as $\pm 10 \%$ of full scale. Data for FY 1983 will circumvent this problem by temperature regulating the incoming gas and the instrument.

Meteorological data. SERI's Renewable Resource Assessment and Instrument Branch [6] installed instrumentation at the Irradiance Research Laboratory (IRL) location to measure standard meteorological variables and furnishes these data to all SERI groups at the field site. Eppley PSP pyranometers and pyrheliometers were used for radiation data. Dew point was measured directly with an electrically cooled mirror. Wind direction and speed were measured at $2 \mathrm{~m}(6.5 \mathrm{ft})$ and $10 \mathrm{~m}(33 \mathrm{ft})$ heights. All IRL data are sampled every two seconds and recorded as minute averages. 


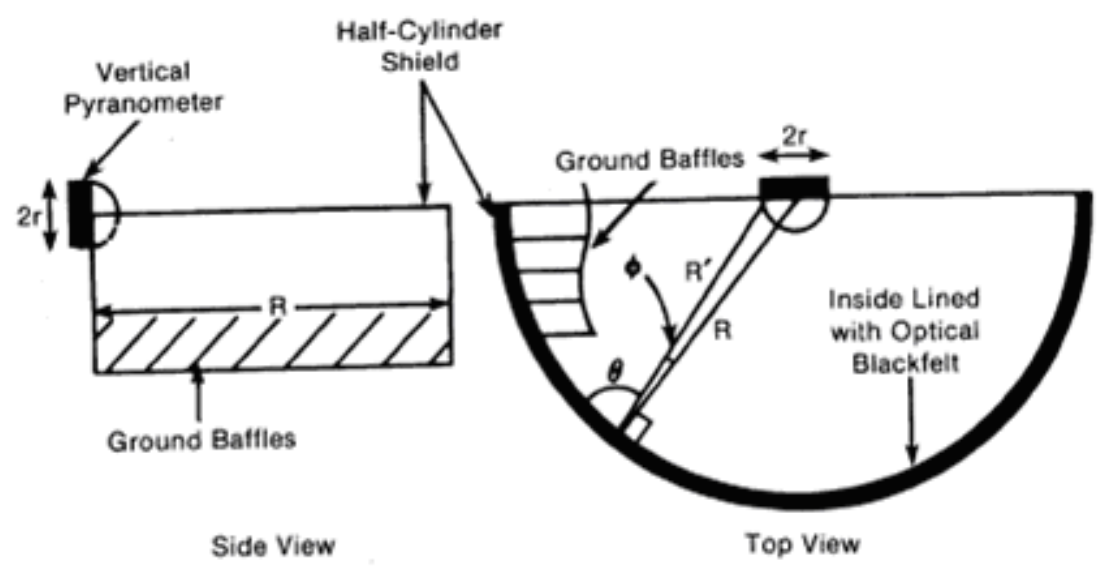

(b) Analysis

Assume: The entire shield interior is black

$$
R^{\prime} \approx R, \varphi-0 . \partial=\pi / 2
$$

Isotropic sky and ground: diffuse fraction $=0.15$

ground reflectivity $=0.8$ (worst case)

$$
\mathrm{r}=0.5 \text { in. }
$$

$$
\rightarrow F_{\text {GETECTOR }}^{\text {Ground }}=\frac{4}{3 \pi} \frac{f}{R}
$$$$
F_{\text {det }-s k y}=\frac{1}{2}-\frac{4}{3 \pi} \frac{f}{R}
$$$$
\rightarrow \frac{G_{A C T U A L}}{G_{I D E A L}}=1+\frac{5.3}{\pi^{2}} \frac{r}{R}-\text { for } 2 \% \text { error } \rightarrow=13 \mathrm{in} .
$$

Figure 6-4. Artificial horizon design

Subsidiary equipment included two new instruments developed at SERI. First, an all-sky flux mapper was developed to measure sky luminance at every point in the sky dome [9]. Second, a $0.3-3 \mu \mathrm{m}$ sky spectrometer is onsite and acquires data on characteristic spectral content of global horizontal and beam radiation in various conditions [10].

Sampling Intervals. Although only hourly averages of certain variables can be compared to code output, the data must be sampled sufficiently often to accurately determine the average. Temperatures and fluxes associated with mass will show slow, nonstochastic variations. In this case, we chose a sampling interval of five minutes as adequate for even extreme conditions of solar driving, since the relevant time constants are at least five minutes.

Several other signals require more careful consideration. First, stochastic variables include wind speed and direction and solar radiation. Consider a worst case where the signal $\mathrm{S}$ is random but equally on and off, so sample standard deviation $\sigma$ is about $0.5 \bar{S}$. Since the error in the hourly 
average is reduced by $\sqrt{ } \mathrm{N}$, where $\mathrm{N}=$ the number of samples per hour, $\mathrm{N}$ is related to a desired fractional error $\mathrm{E}$ as

$$
E_{S}=\sigma / \sqrt{N} \sim \bar{S} /(2 \sqrt{N})
$$

Thus, for a worst case $2.5 \%$ error, $\mathrm{N} \approx 800$, implying a sample rate of about once every 5 seconds.

Second, heater power data must be precise. One approach would be to integrate analogue, and then periodically sample and reset. However, integrating Hall effect watt-hour meters are much more costly than instantaneous watt meters. The problem is that watt meters must be sampled rapidly enough to determine with sufficient precision the on/off time, unless this is computer controlled. Assuming 6 cycles/h, 5 min each cycle, the 5-second sampling interval implies less than $2 \%$ uncertainty and is deemed to be an adequate sampling rate for heater watt meters.

\subsubsection{Data Checking: Zone Energy Balance}

In order to ensure that relevant mechanisms are monitored in sufficient detail, accuracy, and completeness, a zone energy balance will be performed. Although the zone balance, $\mathrm{Q}_{\text {in }}=\mathrm{Q}_{\text {out }}{ }^{+}$ $\delta Q_{\text {stored }}$ is based on the simple law of conservation of energy, application is difficult because of extrapolation from limited flux and temperature data, inaccuracies in the infiltration variable, and uncertainties in solar distribution.

The system for applying conservation of energy is defined to include all mass layers internal to a wall insulation layer. The change in stored energy is then

$$
\partial Q_{\text {stored }}=C_{\text {zone }} \delta T_{\text {zone }}+\Sigma(p c)_{i} A_{i} L_{i}(\delta T i)
$$

Where

$\delta$ indicates net change over some convenient period $\Delta \mathrm{t}$,

$\mathrm{i}=$ wall mass layers,

and other symbols are defined in the nomenclature (Section 6.5).

The $\Delta \mathrm{t}$ would reasonably be chosen as one hour of one day. Note that there is no need to know interior surface film coefficients or the mass and air ratio of absorbed solar flux.

The losses $\mathrm{Q}_{\text {out }}$ can be calculated from data as

$$
Q_{\text {out }}=\int_{\Delta t}\left(\dot{Q}_{\text {skin }}+\rho c \dot{\mathrm{V}}_{\mathrm{inf}}\right) d t
$$

The infiltration term $\dot{V}_{\text {inf }}$ is determined as in Section 6.2.3.1. In the case of the test cell, we will calculate the wall portion as

$$
\dot{Q}_{\text {walls }}=\int_{\Delta t}\left(\Sigma A_{i}\left[T_{\text {in }}(t)-T_{\text {out }}(t)\right] U_{\text {in-out }}\right) d t
$$

This is done because wall losses to the outside cannot be calculated from inside surface fluxmeter readings, since this flux is internal to the system and hence includes both storage and 
losses to the outside. As in Eq. 6-3, we compute losses to the outside based on the $\Delta \mathrm{T}$ across the outer lightweight layers of wall, the insulation or stud layers below the block or sheet rock. The layer $U_{\text {in-out }}$ value is derived experimentally (see Section 6.2.3.1). Using outside-inside surface temperatures, losses to ambient are obtained from data that automatically include both convective and infrared losses at the external surface, eliminating the need to model these troublesome mechanisms.

Energy inputs include heater and other internal hardware, electrical and solar energy absorbed in the zone:

$$
Q_{m}=\int_{\Delta T}\left[\dot{Q}_{\text {heater }}+\dot{Q}_{\text {other }}+\left(\sum_{g=\text { glaz }} A_{g} \dot{Q}_{\text {tran }, g}\right) \alpha_{\text {zone }}\right] d t
$$

Transmitted solar energy is monitored for all glazing types and orientations. The effective cavity reflectance or albedo will be more difficult to estimate when considerable interior mullion blockage exists, as in the SERI test house.

Note that during coheating with the sun blocked, $\mathrm{Q}_{\operatorname{tran}}=0.0$, and $\mathrm{Q}_{\text {in }}$ is more precisely known. The coheating experiment energy balance is correspondingly easier to check under the simplest possible conditions. We expect that the error in the energy balance would be $\sim 15 \%$, limited mainly by the accuracy of fluxmeters $(\sim 10 \%)$ and uncertainties in data extrapolation.

\subsubsection{SERI Field Site Installation}

The cell and the house were prepared for data-taking during late 1981 and early 1982. General instrumentation plans peculiar to each structure are discussed in this section. Detailed instrumentation plans are shown in Burch et al. [11,12]. Since differences exist in the magnitude of the various heat transfer mechanisms, some of the mechanisms are approached differently. A breakdown of mechanisms and the monitoring approach at the SERI site is given in Table 6-6. 
Table 6-6. Measurement Approaches

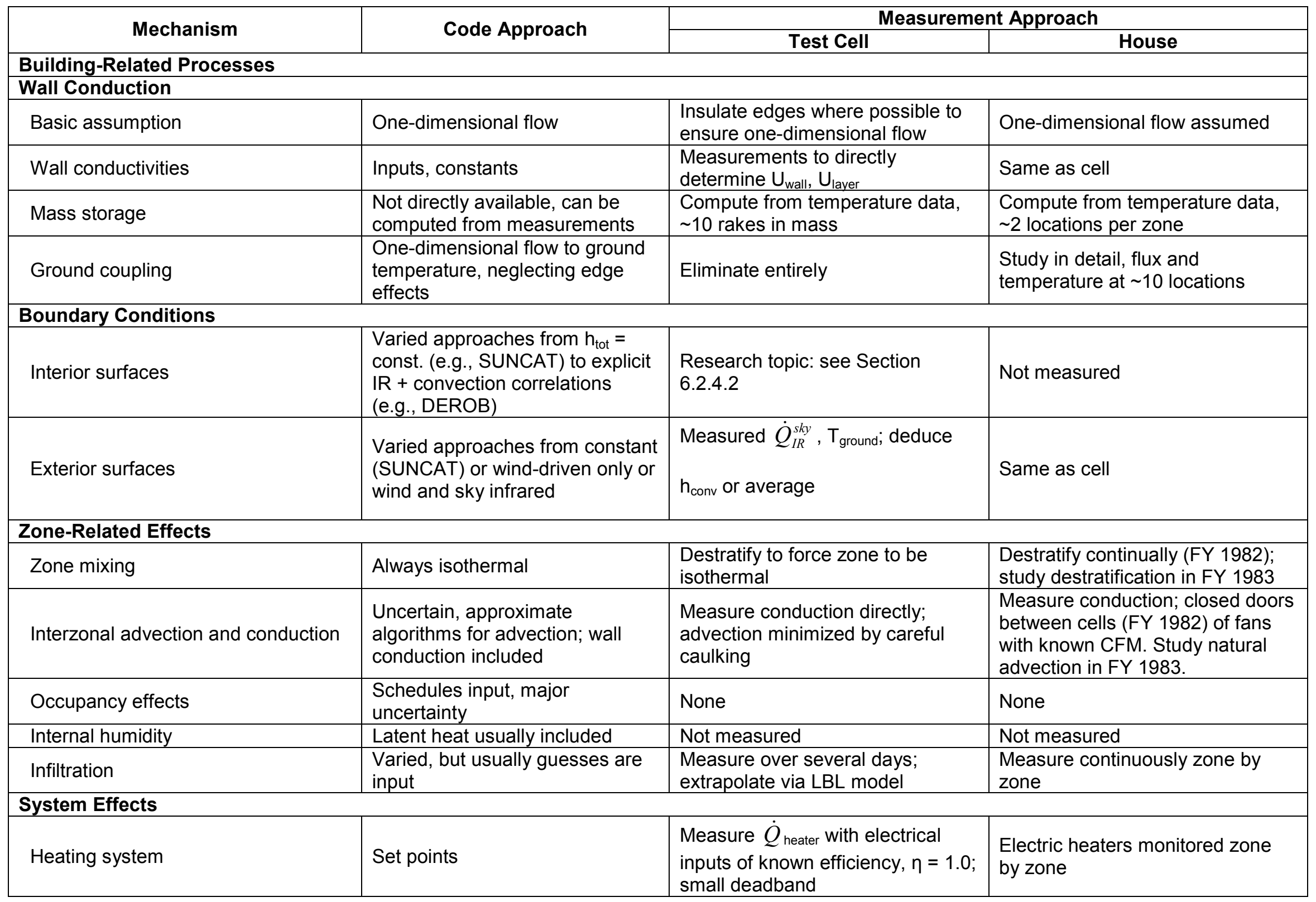




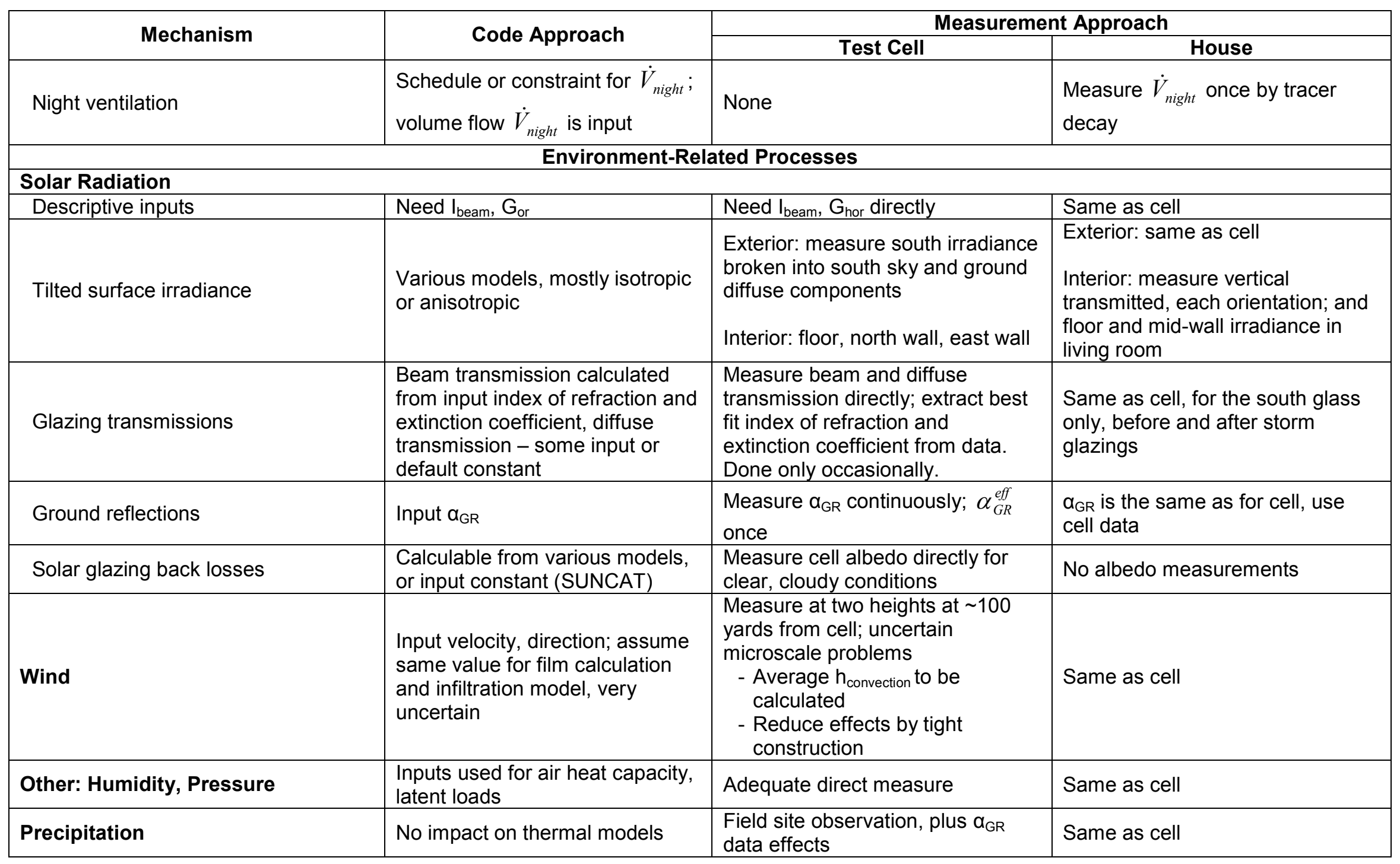




\subsubsection{Test Cell Description}

The test cell is instrumented to focus mainly on the mechanisms of wall conduction (interzonal and outside) and solar heat gain. From the data determination of the cell energy balance can be made to provide a complete, consistent data set. Insofar as possible, all intractable mechanisms are eliminated to provide a case where only mechanisms claimed to be accurately treatable in the codes are included in the problem.

Physical and Thermal Overview. The test cell floor plans and south elevation are shown schematically in Figure 6-la with more detailed construction plans, sections, and other relevant data given in Burch et al. [11]. The cell consists of two zones each about $1.7 \mathrm{~m} \times 3 \mathrm{~m} \times 2.7 \mathrm{~m}(5$ $\mathrm{ft} \times 9 \mathrm{ft} \times 8 \mathrm{ft}$ ), one with low mass and one with high mass. The low-mass zone has standard frame and gypsum board finishing on one side (heat capacity $\mathrm{C}_{\mathrm{lo}} \approx 10^{3} \mathrm{~kJ} /{ }^{\circ} \mathrm{C}\left[500 \mathrm{Btu} /{ }^{\circ} \mathrm{F}\right]$ ). The high mass zone has $\sim 6$ tons of concrete block (heat capacity $\mathrm{C}_{\mathrm{hi}} \approx 6000 \mathrm{~kJ} /{ }^{\circ} \mathrm{C}\left[3000 \mathrm{Btu} /{ }^{\circ} \mathrm{F}\right]$ ). The high-mass glazing is $\sim 35 \mathrm{ft}^{2}\left(\sim 4 \mathrm{~m}^{2}\right)$ giving heat capacity $/ \mathrm{ft}^{2}$ of glazing as $\sim 85600 \mathrm{~kJ} / \mathrm{m}^{2 \circ} \mathrm{C}$ $\left(\mathrm{Btu} / \mathrm{ft}^{2}{ }^{\circ} \mathrm{F}\right)$; the low-mass glazing has been masked with Thermax to make $\sim 17 \mathrm{ft}^{2 \text { glaz }}\left(\sim 2 \mathrm{~m}^{2}\right)$, giving $\sim 200 \mathrm{~kJ} / \mathrm{m}^{2 \circ} \mathrm{C}\left(\mathrm{Btu} / \mathrm{ft}^{2}{ }_{\text {glaz }}{ }^{\circ} \mathrm{F}\right)$. Double-pane glass with 2-cm (0.75-in.) air space was used.

If the cell zones are considered to be isolated from each other and free floating, then the temperature elevation above ambient and temperature swing can be calculated as in Appendix B. In particular, the low-mass cell would stabilize at $\sim 200^{\circ}$ to $22^{\circ} \mathrm{C}\left(\sim 350^{\circ}\right.$ to $\left.400^{\circ} \mathrm{F}\right)$ above ambient average for clear quasi-steady conditions in January, fluctuating $\sim 11^{\circ} \mathrm{C}\left(\sim 200^{\circ} \mathrm{F}\right)$ around the mean. The high-mass zone would float $\sim 33^{\circ} \mathrm{C}\left(\sim 80^{\circ} \mathrm{F}\right)$ above ambient, fluctuating $\sim 8^{\circ}$ to $11^{\circ} \mathrm{C}$ $\left(\sim 14^{\circ}\right.$ to $\left.20^{\circ} \mathrm{F}\right)$, and reflecting high solar gain and large mass. Hence, $\sim 11^{\circ} \mathrm{C}\left(\sim 20^{\circ} \mathrm{F}\right)$ temperature differential between cells will be present at times with considerable interzonal flux $(\sim 150 \mathrm{~W}$ $[1000 \mathrm{Btu} / \mathrm{h}]$ at $\left.11^{\circ} \mathrm{C}\left[40^{\circ} \mathrm{F}\right] \Delta \mathrm{T}\right)$ through the interior partition wall. Hence, interzonal flux is very important in the total cell behavior. Details of these calculations are given in Appendix B.

Zone loads are principally the result of the skin element conduction, dominated by the glazing losses ( $\sim 2 / 3$ of the high-mass load, and $\sim 2 / 5$ of the low-mass load). Film coefficients are clearly important for the glazing conduction analysis, with the glazing U-value changed by $\sim 25 \%$ for winds changing from 0 to $30 \mathrm{mph}$. Infiltration is $\sim 0.03$ changes per hour $(\mathrm{ACH})$, amounting to less than $2 \%$ of the load in either case. Even for rates of $\sim 0.4$ to $0.5 \mathrm{ACH}$, infiltration is still less than $10 \%$ of the load for the high-mass case (see Appendix B).

Elimination of uncharacterizable mechanisms in the cell. We intend to physically eliminate those mechanisms that state-of-the-art codes are known to simulate in a crude fashion or to ignore. First, ground coupling is entirely eliminated by: (1) lifting the cell about $30 \mathrm{~cm}(1 \mathrm{ft})$ off the ground (to decouple conduction to the ground); (2) placing insulation atop the ground beneath the cell (to decouple infrared exchange with the ground); and (3) promoting free air flow underneath the cell while blocking all sun penetration (to ensure simple exchange to ambient temperature). Although ground coupling could be incorporated if desired, it is probably not useful since the geometry of a test cell is different from that of typical structures.

Second, uncontrolled air infiltration is minimized by: (1) caulking external ply joints; (2) using a cardboard-foam liner beneath the external ply as a barrier; (3) tightly fitting bead-board 
insulation into the stud cavities to inhibit air flow at the edges; and (4) carefully caulking the edges of the bead board to the wall studs before plywood or wallboard is applied to the interior. Measurements of infiltration are scheduled as discussed in Section 6.2.3.1.

Third, wall sections were prepared as simple solid conduction paths. Any air gaps in the sections were eliminated since none of the codes treat the dependence of opaque wall air gaps (U-values) on temperatures. The masonry wall edges were insulated to promote one-dimensional flow across the entire wall area, which is assumed by all the codes. Finally, the masonry wall mortar joints were made very thin $(<1 \mathrm{~mm}$ [0.04 in.]) to ensure a homogeneous wall type.

Fourth, advection and stratification effects are eliminated by equalizing the interior air temperature through a monitored low velocity destratifier in each cell zone. We eliminated any significant air flow across interior surfaces, which would alter the convective film coefficient.

Fifth, the cell was painted white on all outside surfaces to minimize the impact of surface absorption on conduction. The area around the test cell includes a test cell to the west, a small building to the north, and a field of trough collectors to the east. These features cannot be input in all the codes, and the white paint will minimize the effects of these complexities.

Test cell instrumentation. Skin conduction is the dominant loss mechanism in the cell. Instruments were installed to allow first law energy balances to be calculated for each major surface in each zone. The typical sensor placement pattern is shown in Figure 6-5. First, temperature rakes and a surface fluxmeter were placed to characterize the thermal behavior of each type of wall. These locations are usually at a surface midpoint, although several other locations were chosen for special boundary conditions (see Burch et al. [12]). From this information, correlations can be made and least squares adjusted for each wall type for surface fluxes and stored energy as a function of previous surface temperature measurements.

Second, each wall has inside and outside surface temperatures measured in a diamond pattern about the center, as in Figure 6-5. This information will be used with the previous correlations to infer the local wall surface flux and stored energy. Hence, relatively precise area resolution is possible to account for variations across the wall surface and to compute an accurate total wall flux. However, wall areas very close to the glass receive the highest solar irradiation, have higher than average surface temperatures, and have largest infrared coupling to the cold glazing surface. Hence, these factors may lead to losses higher than would be predicted by averaging the flux over the entire wall and treating infrared by using an average view factor, or by enhancing the surface-co-zone coupling coefficient. 


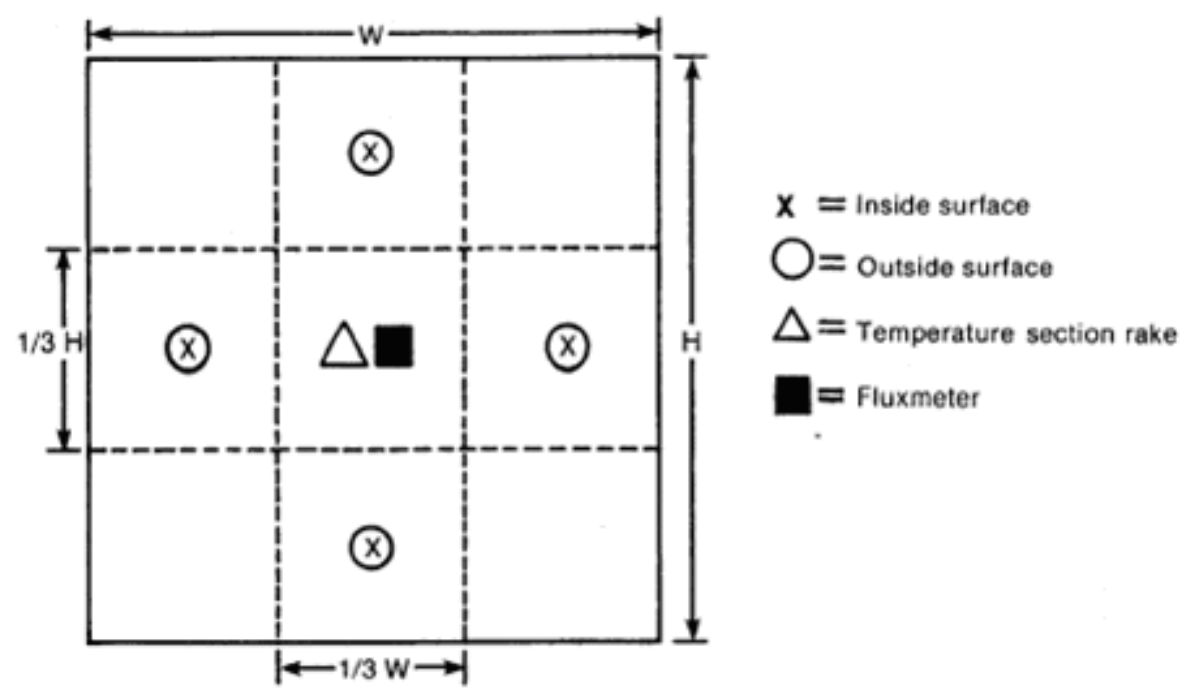

Figure 6-5. Sensor placement pattern

Internal air temperature will be monitored at $1 / 2 \mathrm{H}$, where $\mathrm{H}=$ floor-to-ceiling height, at five locations (Figure 6-3). Additionally, each of the five locations can be examined at $1 / 6 \mathrm{H}$ and 5/6 $\mathrm{H}$ positions to determine stratification. Vertical variation will generally be monitored only at the room midpoint, with other locations monitored only at $1 / 2 \mathrm{H}$. In this way, a true room average air temperature can be calculated, even if a destratifier is not operating. Globe temperature is measured at the zone floor midpoint at a height of $1.6 \mathrm{~m}$ (64 in.).

We considered these relationships plus construction complexities to determine the installation. Burch et al. [11] shows the construction sections and structure measurements and the locations of about 300 sensors. Only 200 channels can be monitored at one time.

\subsubsection{Validation Test House Description}

The validation test house was instrumented to provide accurate system-level data in a realistic but unoccupied house that is comparatively simple and has a basic rectangular shape with uniform construction throughout. System level data emphasize: (1) interzonal coupling by conduction only, or by conduction plus natural advection through doorways (to be studied in FY 1983); (2) ground coupling through the floor and crawl space; and (3) solar gain, using both direct gain and mass wall designs (for FY 1983). Several sets of validation data will be gathered for different configurations of the house. The validation test house floor plan and south elevation are shown in Figure 6-1 with additional information Burch et al. [12]. The house is divided into four destratified zones: two on the south and two on the north. Sealed doors are installed between zones. The house is mounted on a raised foundation, with a l-m (3-ft) sealed crawl space that connects the ground to the floor. The gabled roof has an E-W ridgeline. There are $19.2 \mathrm{~m}^{2}(206$ $\left.\mathrm{ft}^{2}\right)$ glazing, with $8.4 \mathrm{~m}^{2}\left(90 \mathrm{ft}^{2}\right)$ to the south. Shading is substantial, provided by the overhang and mullions on the glazings. The floors are bare hardwood, except that (1) the living room has a nylon carpet with pad for tests before retrofit and a brick layer on a bare wood floor after the retrofit; (2) the southwest bedroom has a brick layer on a bare wood floor after the retrofit; and (3) the kitchen has linoleum over the bare wood floor. 
The building load coefficient for the house before retrofitting is about $26,375 \mathrm{~kJ} /{ }^{\circ} \mathrm{C}(25,000$ $\mathrm{Btu} /{ }^{\circ} \mathrm{F}$ ) per day, which is a fairly large load. Details are available in Burch et al. [12]. Before retrofitting, the frame walls and the floor had no insulation, and the house had numerous air leaks. The economic effectiveness of the retrofits will be analyzed by Barret et al. [13].

Natural air motion is considered by the code authors to be uncertain and was minimized in the house for the first round of validation data. In particular, the air in each was destratified. Interzonal advection was suppressed through closed gasketed doors between zones, and caulking was applied to any potential leaks, including baseboards and electrical outlets. In this way, validation data will attempt to exclude these troublesome mechanisms. However, since a study of advection will provide very useful information on an uncertain energy mechanism, plans are being formed to measure advective air flow in FY 1983 validation data runs.

Instrumentation. We measured energy transport via conduction through each typical wall type at each orientation, including glazings, exterior walls, floor, ceiling, interior walls, ground, and foundation walls. A thermocouple rake and a fluxmeter are installed on at least two heat transfer surfaces of each type. For other orientations or rooms, an inside and outside thermocouple pair is installed from which fluxes for energy balance checking can be calculated using correlations tested at fluxmeter and rake locations. Orientation-dependent factors such as solar loading, infrared coupling, and convection need not be known to infer flux.

Generally, experimental breakdown of the zone energy load through all surfaces is measured directly from fluxes only for the largest south-facing zone, the living room, and for the crawl space. Geometric variation of wall temperature and flux for a given wall will be examined at several locations but not in the detail possible for the smaller cell. Results from the test cell will indicate the importance of spatial variations. Solar flux transmitted through the glazings is monitored for each orientation, E, W, N, and S.

Ground coupling is one of the significant differences between the house and the cell and deserves special study. Sufficient data will be taken to allow continuous energy balance calculations to check measurements from the crawl space for accuracy and completeness. This implies measuring flux and temperature at the floor, the foundation wall, and the ground in addition to considering air exchanges. Detailed plans are shown in Burch et al. [12].

Flux into the ground is directly monitored at five locations, four of which are above temperature rakes. Temperature rakes exist at five other locations, enabling flux and temperature correlations validated at fluxmeter locations to extend flux determination to an additional five locations. Ground flux and temperature profile up to $2 \mathrm{~m}(6 \mathrm{ft})$ in depth are thus well determined.

The inner surface flux and temperature are measured at the middle of the foundation wall for each of the four orientations. Local variations in foundation wall surface temperature are examined at 14 other locations. This information will show significant thermal variations in the wall, if any, and may be used to adjust the energy balance to reflect such variations.

A major facet of the foundation wall conduction is the multidimensional berm and ground conduction. The problem should be two-dimensional except near the house corners. Since the 
codes assume a one-dimensional flow, the user must input some effective length for an ad hoc earth layer. Thus, a two-dimensional temperature rake will be installed in FY 1983 at the north wall to derive isotherms and flux line lengths that can be averaged across the wall. Conductivity can be inferred from path length, $\Delta \mathrm{T}$, and flux. The total wall flux can be compared to that for a wall with an equivalent earth layer that is adjusted to give the best fit to the flux data.

Other aspects of the crawl space energy balance are conduction through the floor, outside air infiltration, and air exchange with the house interior. Flux from the floor is directly monitored at five locations, with surface temperatures measured at five other locations for flux inference. The crawl space infiltration rate will be measured once using the decay curve of a tracer gas, and a suitable model for extrapolation. We expect the air exchange from the crawl space to the interior to be minimal, since the crawl space air is cold relative to inside and buoyancy inhibits the exchange.

\subsubsection{Minimizing External Error Sources: Measurement of Input Parameters}

The major building parameters input to the simulation codes include conductivity and volumetric heat capacity for each layer of the wall, surface solar and infrared properties, glazing optical and conduction properties, zone infiltration, and zone heat capacity. In addition, some codes require input of zone albedo, wall film coefficients, and HVAC and system type. Meteorologic measurements are also required and are described in Section 6.2.3.2.

\subsubsection{Measurement of Building Parameter Inputs}

Zone conduction and total load coefficients are measured in coheating experiments. Coheating holds internal zone temperature constant via electric heaters to measure total load and establish steady interior conditions. Once steady or quasi-steady conditions are attained, wall material conductivities are calculated from in situ temperature and flux data. However, this process presents some difficulties, principally related to steady-state requirements and to wall inhomogeneity (i.e., studs versus insulated cavities).

Coheating and wall properties. If one-dimensional steady-state flow conditions are established, the $\mathrm{U}$-value of any wall element is given as $\mathrm{U}=\dot{Q} / \mathrm{T}$, where $\dot{Q}$ is the steady thermal flux and $\Delta \mathrm{T}$ is the temperature drop across the element. If steady conditions are not present, appropriate time averages must be used as discussed later. U-values must be measured for individual layers, all layers without films, and, if possible, the total wall including the film coefficients.

Attaining steady or quasi-steady conditions. Carefully defined procedures are needed to allow meaningful extraction of wall U-values from in situ coheating data, because transients and changing conditions make the simple instantaneous relation $\mathrm{U}_{\mathrm{i}}=\dot{Q}_{\text {surf }} / \Delta \mathrm{T}_{\mathrm{i}}$ generally inapplicable. The severity of this problem depends on the wall layer time constants. For the glazings, for example, the time constant is much less than an hour and the steady-state condition presents little problem. For the cell mass walls, however, the time constants are on order 24 hours, and one cannot expect ideal steady conditions over several days. One solution to this problem is based on use of time-averaging. Consider the problem shown in Figure 6-6. 


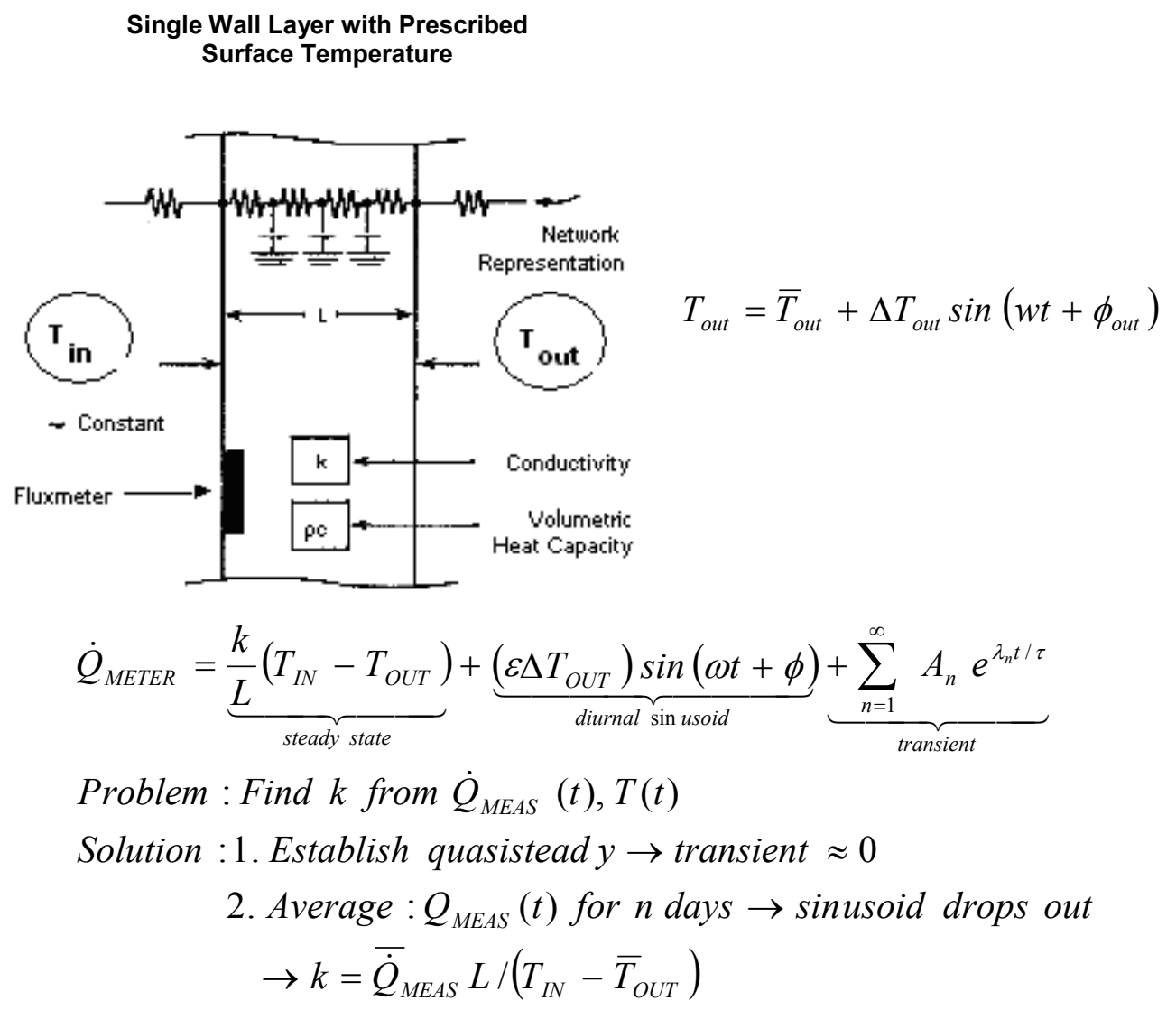

Figure 6-6. Coheating data analysis

Analyzing a single layer in which the outside surface varies sinusoidally and the inside surface is fixed, the inner surface flux is a sum of three parts: (1) a constant; (2) a sinusoid of the same frequency as the driving force (diurnal in this case); and (3) a transient in the form of an infinite sum with terms $A_{n} \simeq \alpha_{n} e^{-\lambda n t} \tau$, where $\tau=$ layer time constant. The transient terms are negligible after two or three time constants. This is one of the criteria to use in defining quasi-steady conditions, where quasi-steady conditions mean that all parameters are periodic with a time period of 24 hours. If quasi-steady behavior is present and the temperature difference and flux are averaged over an integral number of days, the sinusoid term drops out, and the $\mathrm{k}$ value is extracted as in Figure 6-6. Hence, if the diurnal time-averaging approach is used, the problem demands only that quasi-steady conditions have been established.

Presence of quasi-steady conditions can also be verified experimentally to some extent. Taking an average over a day, the midpoint temperature of any wall layer must be the mean of the layer edges. All the concrete block and Styrofoam temperature rakes have thermocouples positioned at the mass center, expressly to verify the existence of quasi-steady conditions. Finally, the experiment temperature and flux time histories should directly indicate repetitive diurnal cycles.

Any quasi-steady period can be used to perform this analysis for wall layer conductivity. However, a major storm accompanied by low heavy clouds for about three days would provide the largest $\left(\mathrm{T}_{\mathrm{in}}-\mathrm{T}_{\text {out }}\right)$ and the minimum $\Delta \mathrm{T}_{\text {out }}$ daily ambient temperature swing. Also, when 
heavy clouds are present, the infrared sky temperature approaches ambience and presents less difficulty in determining the outside convective and radiative coupling.

The second solution to the problem of extracting conductivity from in situ data is more general but requires use of a thermal circuit model, as in Figure 6-6. An n-node model for a onedimensional, multilayer wall is easily input with site-measured boundary condition data using, for example, the versatile network code MITAS [14]. It is then straightforward to find the best fit parameters $k_{i},(\rho c)$, where $i=$ layers of wall in a least squares sense. The one-dimensional thermal circuits are appropriate here and are considered to be as trustworthy as the timeaveraging approach [15].

Other techniques can be used to determine wall properties. These more standard techniques will demonstrate the accuracy of the above approaches. First, samples can be sent to a reputable laboratory equipped with an ASTM guarded hot plate or similar equipment for determining conductivity. Second, guarded box method can be implemented. This method is detailed in ASTM C236-66 [16], and efforts are being made to achieve such measurements in FY 1983. To ensure steady interior temperature in the insulated test cell during coheating runs, the sun should be excluded from the cell interior. Although for extracting coheating data this is possible with removable shades, the glazing conduction properties would be changed during the day. The data gathered during the coheating run using removable shades could not be used for any building validation purposes other than for providing U-value data. We chose to paint the glazings with white paint to keep the conduction properties unchanged throughout the entire day.

Framed wall inhomogeneity. A wood frame wall presents related problems both in defining model calculations and in analyzing measurements. The codes use various wall conduction methods, all based on assumptions of one-dimensional symmetry for each wall. Since this is inconsistent with actual study and cavity construction, the inputs must be correspondingly adjusted to give a good account of overall behavior. For a given frame wall, one can: (1) input a single wall, with an area-averaged stud and cavity conductivity and beat capacity; or (2) input two walls with the studs aggregated as an input wall separate from the insulated cavity portions and with the combined area of the stud and the cavity wall being the same as in the given frame wall. The differences in these two calculation approaches are minimal, but different inputs are required.

The measurements of stud U-value are complicated by the change in thermal properties across the stud, which induces some two-dimensional effects on flow (Figure 6-7). If the flow were entirely one-dimensional through each different cross section, temperature profiles as shown in Figure 6-7a would result. The gypsum sheathing, however, will not support large temperature gradients, and the flow will adjust as shown in Figure 6-7b. Thus, attempts to measure the stud conductivity directly using fluxmeters and temperatures can be misleading.

Four solutions exist for this problem. First, handbook values or estimations can be used for wood conductivity. Second, if a section has solid studs, then the U-value for studs can be obtained using $\dot{Q} / \Delta$ T and measured values. The parallel path calculations can then be used to derive average conductivity for the area, since the exchange between stud and cavity does not significantly change the total flux. The solid stud's dimensions must be at least twice the stud 


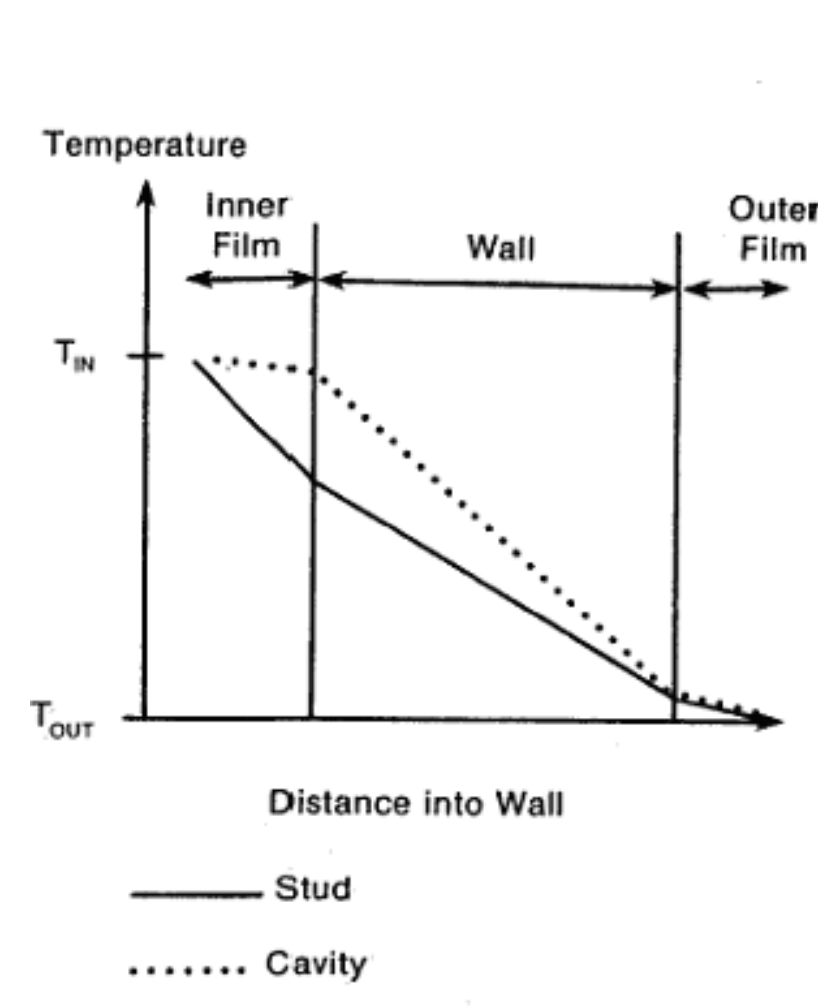

(a) One Dimensional Temperatures

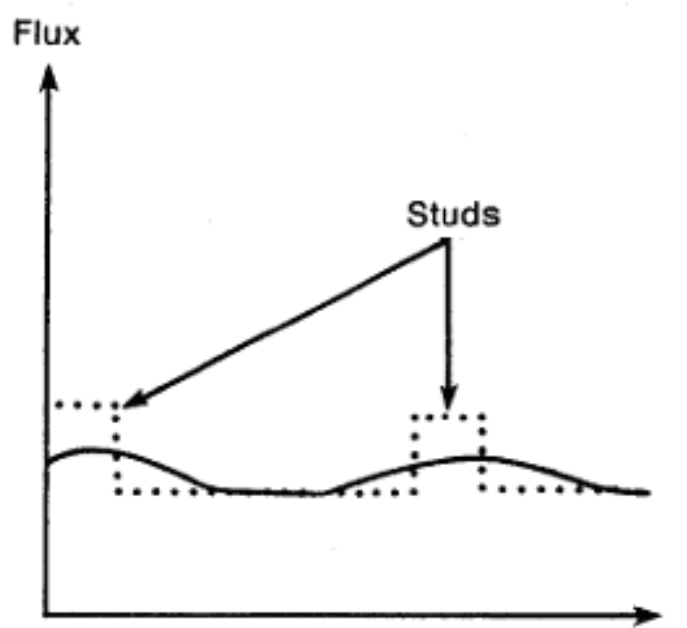

Distance Along Surface

-Two-Dimensional

....... One Dimensional

(b) Surface Fluxes

(c) Linear Fluxmeters

Figure 6-7. Frame wall characterization 
thickness and taken at the central location. Third, the wall flux can be directly measured as a composite unit using an appropriate fluxmeter, as in Figure 6-7c. The sensor length can be either equal to the stud spacing (placement not critical) or one half of the stud spacing (one end must be precisely at stud midpoint), relying upon expected symmetry. As an alternative, three or four fluxmeters (placed close together where flux variation is largest) can be used; each reading must be weighted by the appropriate fraction of total area that experiences that flux. Fourth, the guarded hot box method can be used, although, as specified in [16], it provides no separate information on studs or insulation material.

For the test cell, the second of the above approaches will be used, since a large area of solid studs is present around the door frame and will be used to derive the stud U-value directly. However, the third approach will be used for the house. Before insulation, the nearly isothermal air gaps in the location between studs will lead to a disruption in stud flow. For best attainable accuracy, the wall U-value should be measured as in Figure 6-7c, and the wall characterized as having an average U-value, area-averaged by the fluxmeter. Consistency in the methods will be examined by comparing with the guarded box results. In this way, the spread of wall parameter values obtained by different techniques will help define the experimental error in the important wall inputs.

Infiltration data. The effect of infiltration is different for the test house and the test cell. Although infiltration is much less than $10 \%$ of the total cell load (see Appendix B), it is still important and should be determined with reasonable accuracy. The LBL infiltration model will provide cell infiltration hourly rates [17] normalized with tracer gas decay data. The tracer gas data give average values of the infiltration rate for specific time intervals of several days, accurate to about 5\%. The LBL pressure model can be normalized in several possible ways using the tracer gas results and applied with some confidence over longer durations to generate hourly rates. Tubes were inserted through the north wall of each zone into the destratification flow to avoid opening the door (and hence significantly disturbing infiltration) for sampling. Without renormalization, the LBL procedure was $\sim 20 \%$ uncertain [17], which should be an upper limit for error here. Even at 20\% uncertainty, the net load error in the cell caused by infiltration error is much less than $2 \%$.

House infiltration measurements are crucial to deriving validation data, since in this case infiltration is a major piece of the energy budget. We will measure infiltration zone by zone, since rates can differ significantly between zones. The system used is shown in Figure 6-8. The site MODCOMP minicomputer will initiate: (1) the controlled injection of $\mathrm{SF}_{6}$ gas through solenoid valves to each zone whenever the zone tracer gas reaches the concentration $C_{j}(t)<C_{\text {min }}$, the chosen IR analyzer detection limit; (2) sampling of the air from each zone through a closed air loop for measurement with the IR analyzer; and (3) digitization of the analyzer output and subsequent data handling.

Two kinds of data can be taken. First, tracer gas decay of a given zone can be monitored, as in Zone 1 of Figure 6-8. Second, the system can monitor buildup and decay of $\mathrm{SF}_{6}$ level in Zone $\mathrm{j}$ when Zone $\mathrm{k}$ is injected with gas from which interzonal convection can be inferred. We expect that interzonal rates will be small when the gasketed interzonal doors are closed. 


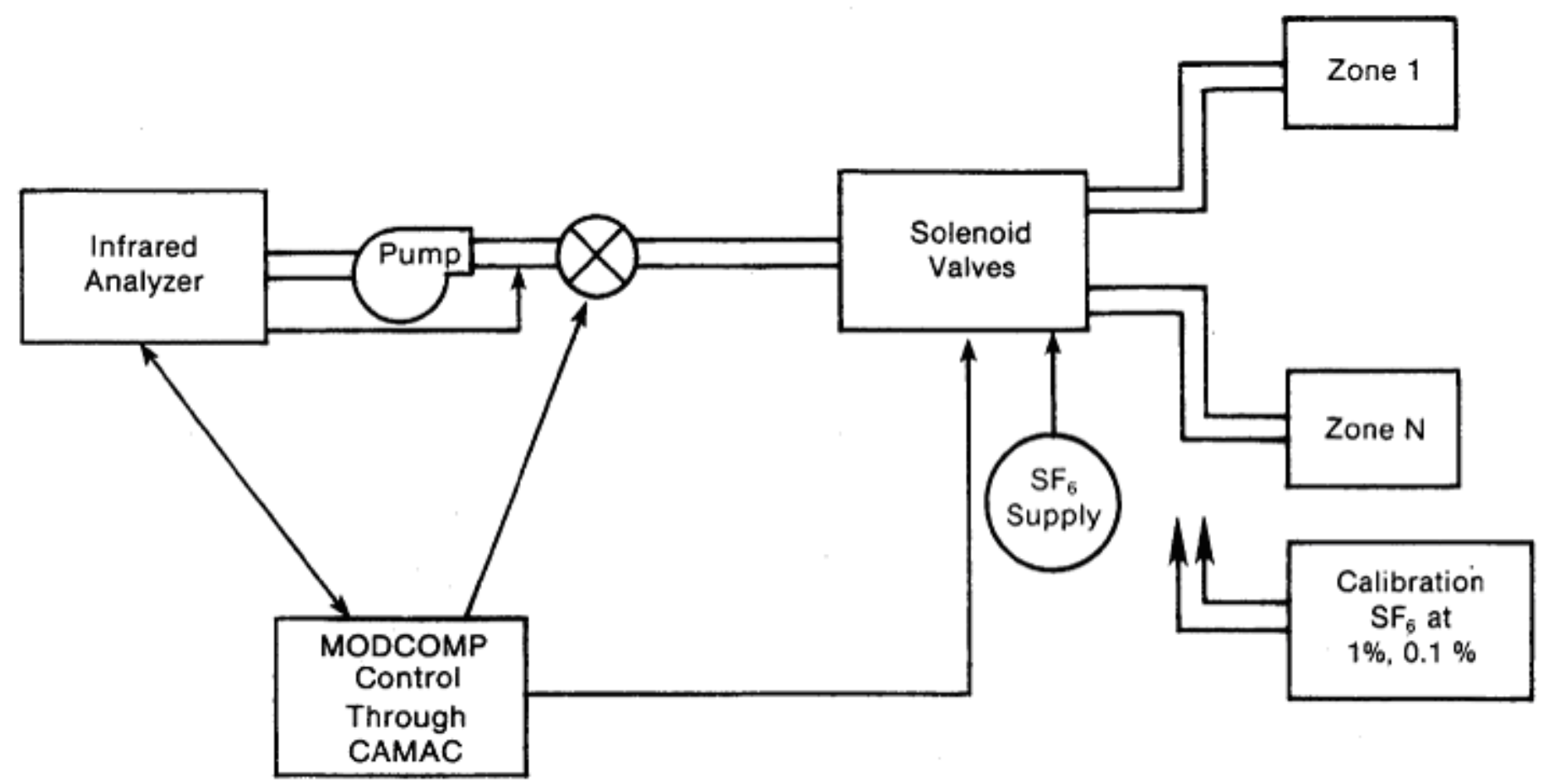

$\mathrm{C}_{\text {MIN }}=$ Minimum Detectable $\mathrm{SF}_{6}$ Level

Figure 6-8. Schematic infiltration measurement 
Generally, each of the four interior zones will be monitored continuously with injection and decay cycles. Thus, correlations can be developed to give the measured infiltration rates as a function of conditions, based on some reasonable assumed form such as

$$
\dot{V}_{\text {inf } j}=A_{1 j}+A_{2 j} \bar{V}_{\text {wind }}^{2}+A_{3}\left(\bar{\Delta} \bar{T}_{\text {in-out }}\right)_{j} .
$$

Finally, dependence on wind direction can be considered, if enough data are available under a variety of approximately steady incidence angles.

Opaque surface optical properties. Optical properties of all opaque surfaces are measured directly using solar band or infrared band reflectometers on at least two samples of each surface. Values are listed in Burch et al. [11,12]. These data eliminate the need to estimate absorptivities from qualitative description. These data were gathered using SERI Geier-Dunkel reflectometers that were calibrated before and after sample measurements. Derivation of values for glazing optical properties are discussed in Section 6.2.4.1.

Zone albedo. One of the codes considered for validation (SUNCAT) requires input of the zone solar back-loss coefficient, which is $\rho_{z}=$ zone effective reflectance or albedo. Further, actual absorbed energy is $\alpha_{z} Q_{\text {in., }}$, where $\alpha_{z}=1-\rho_{z}$. Although $\rho_{z}$ can be calculated, we can measure it directly as $\rho_{z}=\dot{Q}_{\text {out }} \dot{Q}_{\text {in }}$. The process used is shown in Figure 6-9. Since energy input to the zone is given as $\dot{Q}_{\text {in }}=\mathrm{A}_{\text {glaz }}\left(\mathrm{G}^{\text {tran }}\right.$ vert, one needs to measure $\dot{Q}_{\text {out }}$ with a specially baffled pyranometer, shown in Figure 6-9. The baffle prevents any substantial view of the sky, either directly or through reflection from the second glazing. The baffle must be sized so that for photon incident angles less than some limiting incidence angle $\Theta_{\text {cut }}$, no reflections will reach the pyranometer (see Eq. 1, Figure 6-9). This leads to $L=43 \mathrm{~cm}$ (17 in.) for $\Theta_{\text {cut }}=80 \mathrm{deg}$. Any location dependence of $\mathrm{Q}$ will be investigated and averaged out.

Several corrections are necessary to $\dot{Q}_{\text {out }}$ and $\dot{Q}_{\text {in }}$ in the $\alpha_{\mathrm{z}}$ equation. First, since the baffle area $A_{\text {baf }}=0.14 \mathrm{~m}^{2}\left(1.58 \mathrm{ft}^{2}\right)$, it is clear that this can lead to substantial blockage of glazing area; this must be corrected. One approach is that the glazing area for calculating $\dot{Q}_{\text {in }}$ should be an effective area, $\mathrm{A}_{\text {eff }}=\mathrm{A}_{\mathrm{glaz}}-\mathrm{A}^{\mathrm{ba}}$. Second, back-reflected radiation absorbed in the glazings should not be considered as entirely absorbed in the zone. Rather, $\dot{Q}_{\text {out }}$ should be increased by the amount $\dot{Q}_{\text {lost }}$ based on a one-dimensional steady-state flow argument. Zone albedo measurements will be taken several times throughout the day on a clear and an overcast day, once in fall or spring, and once in winter.

Ground reflectivity. Data are also being taken to define ground reflectivity, using an Eppley Model PSP as in Figure 6-10. Ground reflectivity will vary with precipitation and snow cover, and, since codes accept only constant values, suitable averages (probably over experiment runs or over monthly intervals) will be prepared from the data. 

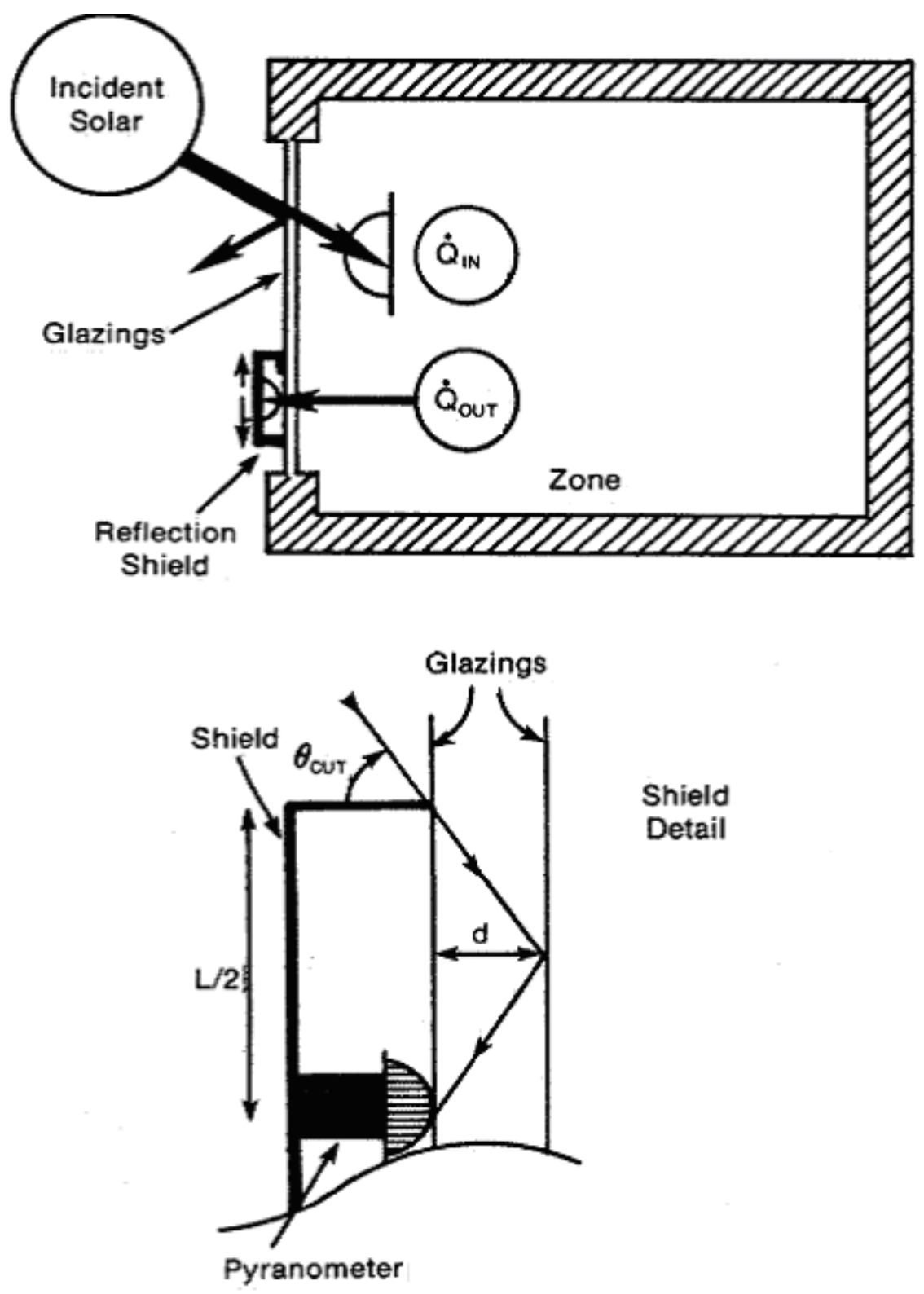

If $\theta_{\text {cut }}=$ Limiting angle

then $1 . L / 2=2 d \cot \left[\pi / 2-\theta_{\text {cut }}\right]$

2. $\dot{Q}_{\text {out }, \text { TOTAL }}=\dot{Q}_{\text {OUT }}+\dot{Q}_{\text {LOST }}$

where $\dot{Q}_{L O S T}=$ Back reflected radiation

absorbed in glazings

that is lost to the outside

Figure 6-9. Solar Radiation Measurements A 
Building codes generally neglect the effects of reflections off building external surfaces. Reflections off east, west, and south walls will strike and reflect from the ground and case additional gain to the building surfaces, as in Figure 6-10(a). Combining ground reflection data from "farfield" and near the building, it is possible to estimate an "effective" ground reflectivity that accounts for this added radiation, as indicated schematically in Figure 6-10(a). Results of code calculation of ground diffuse with measured and effective $\mathrm{P}_{\mathrm{GR}}$ can be checked against data by using an inverted horizon shield, as in Figure 6-10(b).

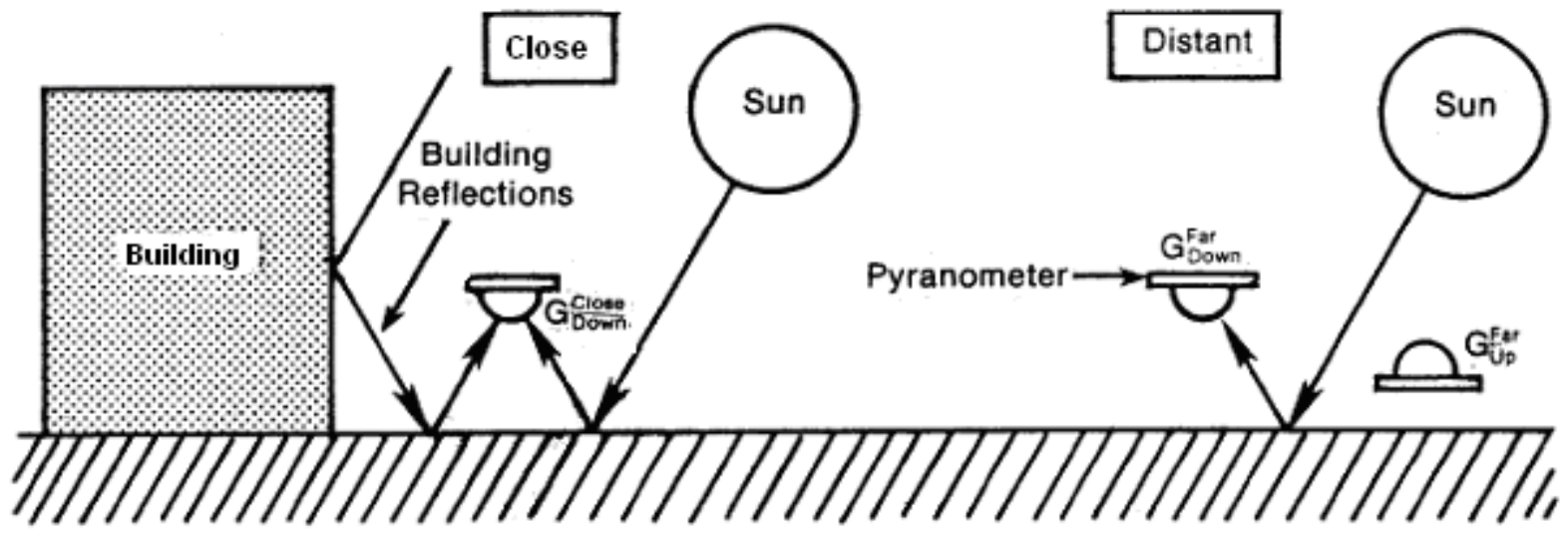

(a) Measurement of Ground Albedo

True Ground Albedo $\rho=\frac{G_{D o w n}^{F a r}}{G_{U p}^{F a r}}$

Apparent Close Albedo $\rho^{\prime}=G_{\text {Down }}^{\text {Close }} / G_{U p}^{\text {Far }} \sim \rho\left[1+\rho_{\text {BUILDING }} F^{1}\right]$

Effective Ground Albedo: $\rho_{\text {eff }}=f_{1} \rho+f_{2} \rho^{1}, f_{i}=$ weighting factors

Ground Diffuse $=F_{\text {bldg-grd }}\left(\rho_{\text {eff }} G_{U p}^{\text {Far }}\right)$

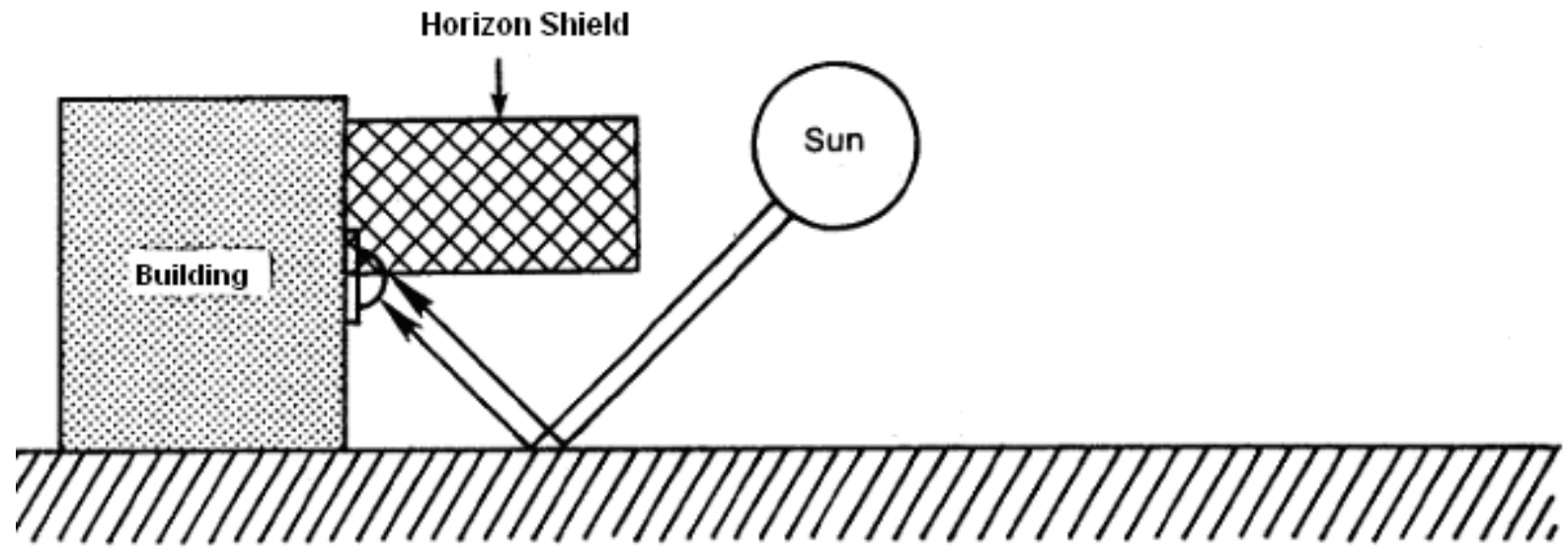

(b) Measurement of $G_{\text {Vert }}^{G R}$ Diffuse Ground on Vertical

Figure 6-10. Solar Radiation Measurements B 
Other parameters: specific heat. The specific heat of building materials will be directly measured using calorimetry, and compared to values derived from the analysis of Section 6.2.3.1 using the thermal circuit model parameter extraction.

\subsubsection{Environmental Data Inputs}

The second class of external error is predominantly the weather data, divided into general meteorologic and solar data. General meteorologic data include: (1) fully shielded ambient

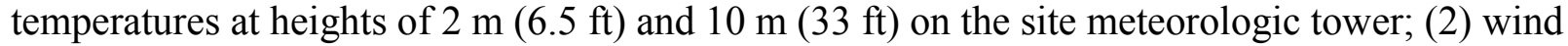
direction and velocity at two heights; (3) barometric pressure; and (4) dew point. These data are maintained and provided by SERI's Renewable Resource Assessment Branch [6]. Additional validation data include wind direction and velocity closer to the house, ambient temperatures near the cell and house surfaces, and infrared sky temperature. These latter data are necessary for checking external infrared coupling mechanisms and will directly point out the necessity of including this mechanism to determine roof fluxes to better than 10\% [2]. Ground temperatures about $2 \mathrm{~m}(6.5 \mathrm{ft})$ from typical walls are monitored so the infrared exchange temperature for vertical walls can be adequately calculated as a linear view-factor-weighted sum over ground, ambient, and sky temperatures.

Solar radiation inputs required by the radiation processors in the codes consist of total global horizontal and direct normal beam data (see Figure 6-11). The global horizontal data are provided by the Resource Assessment Group installation at our field site, using Class 1 Eppley PSP pyranometers. Uncertainty in the beam/diffuse split and its effect can be considerable. For example, various correlation procedures (with attendant uncertainty) have been used to derive the diffuse fraction, as in SOLMET and TMY file preparation or some previous code validation [18]. For our data, the normal beam is directly measured using an Eppley normal incidence pyranometer and a microprocessor-controlled tracker [6]. This method is superior to shadow band diffuse measurements, since the shadow band correction is uncertain by about 5\% [19].

The environmental data are given to the codes in TMY format files. No direct data are being taken on the following TMY meteorological variables: ceiling height, cloud cover, visibility, or precipitation. Presence of snow cover will be inferred from the albedo data. Cloud cover is used in one of the codes (DOE 2.1) and can be calculated during the day from the radiation data using the inversion of the SOLMET correlation (Dodge City coefficients) relating cloud cover to a third-order polynomial in the ratio between measured global horizontal insolation and calculated clear day values [6]. The other data fields are not used in any of the code thermal models but will be filled with the appropriate missing data code when the TMY format files are prepared.

\subsubsection{Mechanism Isolation}

Operation of heat transfer mechanisms in the structures can be isolated by direct measurement of appropriate quantities and by sequencing experiments from the simple to more complex. This section first discusses plans for isolation of the important solar mechanisms. Next, a proposed method for determining the film coefficient is considered, isolating the convection coefficient value using a technique involving temporary use of a low-emissivity foil coating. Finally, the experiment plans are detailed, pointing out the significance and use of the proposed data runs. 


\subsubsection{Solar Processing Isolation}

As shown in Figure 6-11a, the codes require inputs that define the beam and diffuse split. Any two of the three radiation variables (normal beam $=\mathrm{I}_{\text {beam }}$, sky diffuse on horizontal $=\mathrm{G} G_{h o r}^{\text {dif }}$, and global horizontal $=\mathrm{G}_{\mathrm{hor}}$ ) are sufficient to extract the third remaining variable, as given by Eq. 1 of Figure 6-11a. From this information on the solar environment, the codes derive the following intermediate solar fluxes: (1) three components of global irradiance on external surfaces of various orientations: (a) beam $\mathrm{G} G_{\text {surf }}^{\text {beam }}$ (b) sky diffuse $\mathrm{G} G_{\text {surf }}^{\text {dif }}$, and (c) ground diffuse components $\left(\mathrm{G} G_{\text {surf }}^{g r}\right)$; (2) beam and diffuse glazing absorption and transmission; and (3) global irradiance $\mathrm{G}$ on interior surfaces of various orientations (solar distribution). 
(a) Input Information

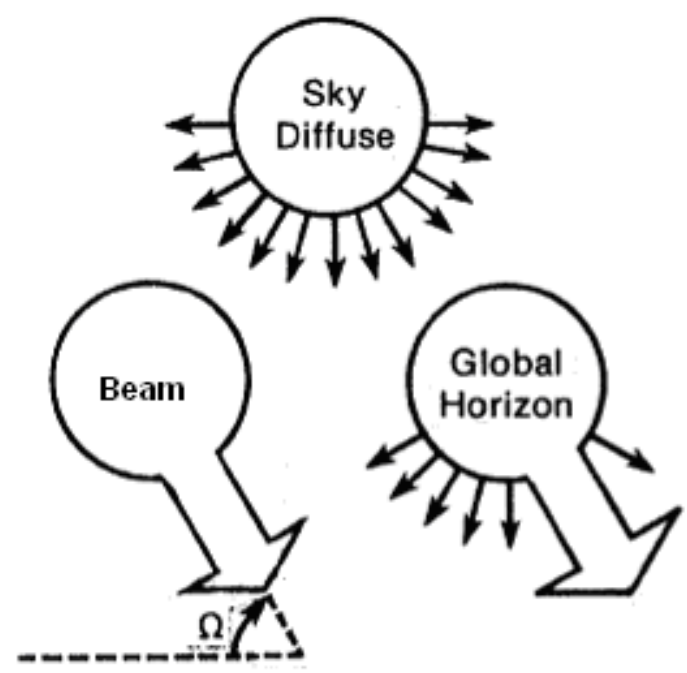

1. $G_{\text {HOR }}=I_{\text {BEAM }} \sin (\Omega)+G_{\text {HOR }}^{\text {DIF }}$ (b) Derived Information

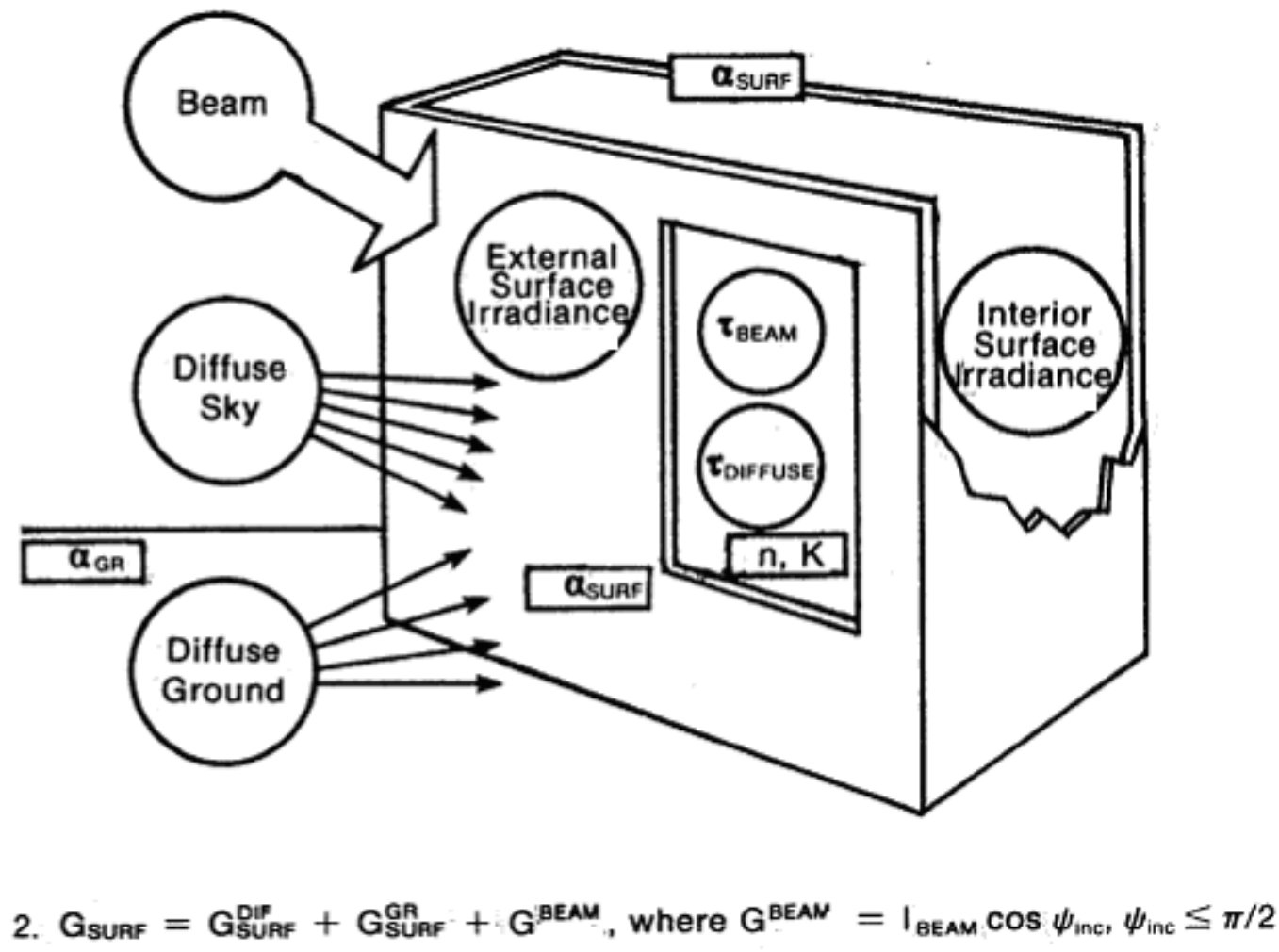

Figure 6-11. Radiation processing 
The external surface beam problem is the simplest. Note that the beam problem simplicity and the accuracy of the SERI beam data lead to confidence in extracting diffuse irradiances by subtracting beam contributions from measured global irradiances.

The diffuse problem is more difficult, because it emanates from all points of the sky in a complex, weather-dependent, nonuniform fashion. Most codes include radiation processors that assume that the sky radiance $\mathrm{R}_{\text {sky }}$ is uniform in all directions (see Section 7.0). Given such a uniform $\mathrm{R}_{\text {sky }}$, sky diffuse radiation on any orientation can now obtained by direct integration or view factor usage. However, $R_{\text {sky }}$ is not isotropic for the following reasons: (1) aerosol scattering is highly peaked toward small angles, giving higher radiance in sky positions near the sun that scatter at small angles to the receiver; (2) the sky is brighter near the horizon, since scattering intensity at any angle is approximately proportional to the number of scattering centers in that direction (limb brightening caused by air mass effect); and (3) clouds are complex scattering elements. These effects are clearly visible and are noted in Figure 6-12 [11]. DOE-2.1 has options that include part of (1) and (2) above, although it is difficult to obtain documentation on exactly what model is employed.

Pyranometer Data. We intend to provide data that will determine the radiation processor errors with two measurement approaches: pyranometer data and sky radiance mapping. Pyranometers will be used to measure: (1) global irradiance on east and north vertical external surface of the cell (data only occasionally); (2) global irradiance on south vertical (continuously); (3) irradiance from sky diffuse plus beam only, on south vertical, using a Resource Assessment PSP pyranometer baffled with artificial horizon (continuously); (4) irradiance from the ground on a south vertical surface, using a baffled pyranometer (occasionally); (5) global irradiance on the floor and the north wall inside the east zone of the test cell (continuously); (6) irradiance on floor and north wall of house in the living room (continuously); and (7) vertical transmitted radiation for the glazing, for each orientation (one in the cell, four in the house).

Component breakdown of $\mathbf{G}_{\text {verts }}$ into $G_{\text {vert }, s}^{\text {beam }}$ and $G_{\text {vert }, s}^{g r}$. Subtraction of the beam component from the shielded vertical irradiance data gives sky diffusion on vertical south surface; i.e., $G_{\text {vert }, s}^{\text {dif }}=\mathrm{G}^{\mathrm{sky}}$ vert,s $-\mathrm{I}_{\text {nbeam }} \cos \psi$. This value can be directly compared to the code prediction for sky diffuse irradiance, if available in output. $\mathrm{G}^{\mathrm{gr}}{ }_{\text {vert } \mathrm{s}, \mathrm{s}}$, the ground-reflected component, comes from measurement (4) in the above paragraph. It can also be derived by: (a) subtracting (3) from (2); or (b) computing from $\alpha_{\mathrm{gr}}$ and $\mathrm{G}_{\mathrm{hor}}$ using view factor theory. Agreement between these methods gives another example of a direct measure of the analysis consistency and accuracy. $G_{v e r t, s}^{g r}$ is also directly comparable to code computations. This detailed south vertical irradiance breakdown with the north and east vertical surface total irradiance data will provide complete checking of the external surface global irradiance algorithms in the codes.

Glazing optical properties. The glazing transmission problem is shown in Figure 6-13, indicating two vertical pyranometers and a tracking pyrheliometer. The pyrheliometer data will provide the beam transmission $\tau_{\text {beam ( } \psi)}$ directly, as shown in Figure 6-13, Eq. 2. Once $\tau_{\text {beam }}$ is known, the energy balance equation (Figure 6-13, Eq. 1), can be solved for $\mathrm{T}_{\text {vert, }}^{\text {dif }}$, the diffuse vertical transmission factor. The diffuse transmission is an average over angles, weighted by transmission, sky and ground radiance, and area projection (see Figure 6-13, Eq. 3). The vertical 
diffuse transmission is typically assumed by the codes to be an input or default constant. The data will yield the best fit constant to be input to the code. However, it can be expected to vary somewhat with sun position. The data will indicate the extent of variation, how serious the error is if a constant value is employed, and whether a monthly declination correlation can improve the diffuse transmission calculation.

In some codes, index of refraction $\eta$ and extinction coefficient $\kappa$ are used to derive glazing transmission and absorption characteristics using electromagnetic theory. It can be difficult to get

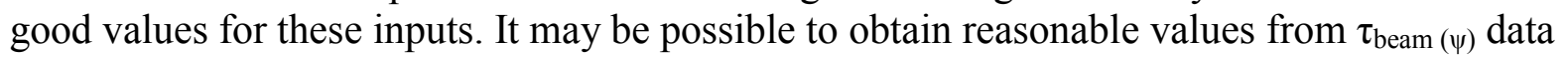
using the well-known $\tau_{\text {beam }}(\psi, \eta, \kappa)$ from electromagnetism. The results would not include effects of dirt on the surfaces (see Section 6.2.1.1 and Figure 6-3).

Sky mapper data: $\mathbf{R}(\Theta, \varphi)$ values. The second approach to defining the diffuse irradiance on tilted surfaces is more general but less direct and with more measurement error. Depending on budgets, an all-sky radiance mapper developed at SERI [9] will be either on the site continuously with daylighting and performance test groups, or available for several days only. The instrument uses an orthographic projection lens and other front-end optics to project the sky dome onto the plane of a silicon photodiode vidicon. The video image is digitized and stored in an 8-bit memory, having $256 \times 256$ spatial resolution. After applying a calibration constant, the data are stored for later processing. Preliminary examples of this kind of data are shown in Figure 6-12 [11].

The instrument can be calibrated to give photoptic quantities (luminance) or radiation quantities (radiance). In the luminance case, appropriate filters are used to tailor the net response to the standard response curve. Like any spectrally sensitive detector, however, problems exist in the radiance calibration. Even if an appropriate spectral filter is used to flatten the detector response in the silicon detector range of wavelengths (about 0.3 to $1.1 \mu \mathrm{m}$ ), one must make some assumption about the ratio of radiation intensity detected to that outside the detector response interval. This ratio (and hence the radiance calibration factor) depends on the spectral distribution of the radiation from the sky, and can be $\pm 20 \%$ considering the clear sky and total cloud extremes [4]. Hence, these fundamental calibration problems point to a different calibration factor for clear or cloudy conditions, with a larger error for partly cloudy conditions. In particular, shielded pyranometer data, with the shield at adjustable angles, will provide the data crosscheck needed. 
Location:

Date:

Time:

Solar Altitude:

Solar Azimuth:

Sky Condition:
Date:

Time:

Solar Altitude:

Solar Azimuth:

Sky Condition:
Golden, Colorado

27 June 1981

11:00 a.m. MST

69 deg

-42 deg

Mostly clear with low clouds to NW and SW

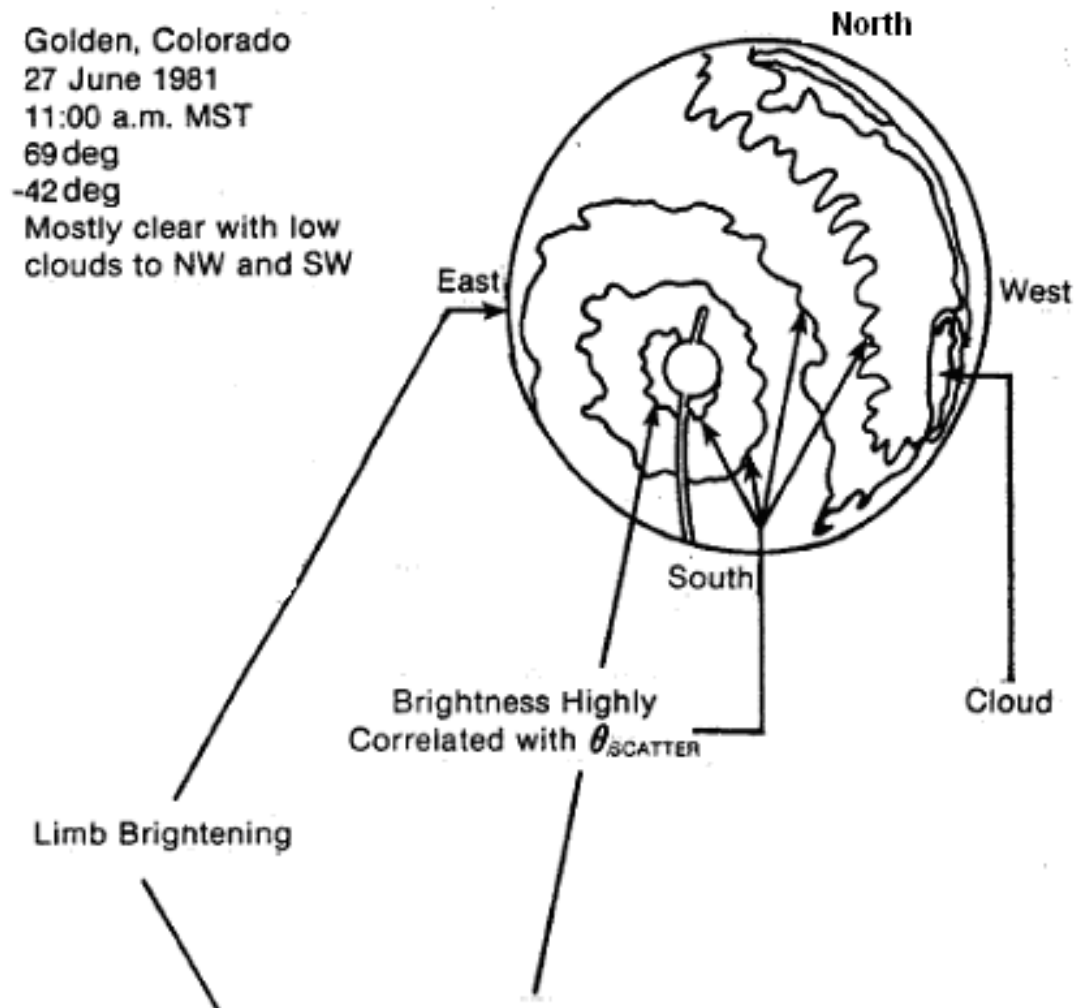

rightness Highly

Peaked at Smail $\theta_{\text {scatren, }}$

Near sun

27 June 1981

12:00 Noon MST

$73 \mathrm{deg}$

0 deg

Mostly overcast, dark

thunderhead to NW and SW

East

North 


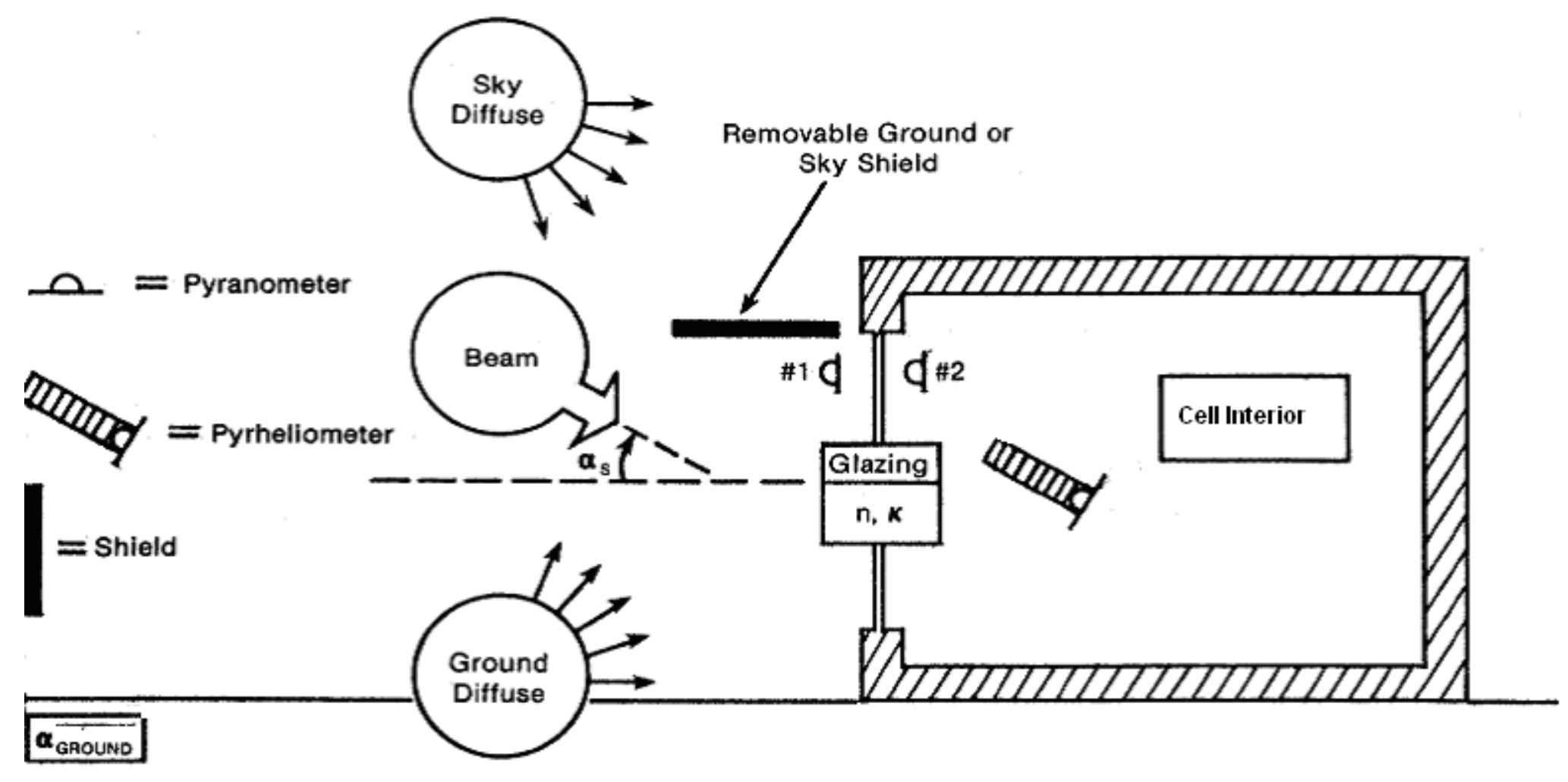

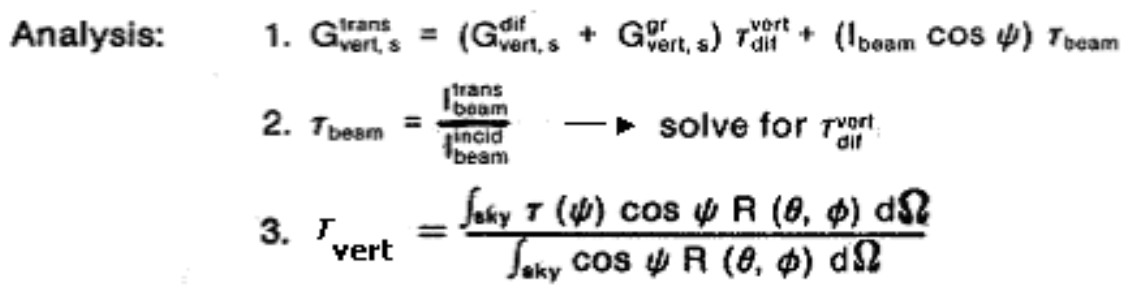

Figure 6-13. Glazing transmission 
Given sky radiance $\mathrm{R}(\Theta, \varphi)$ directly from the sky mapper data, one can use the following equation to derive sky diffuse irradiance on any surface:

$$
I_{\text {surf }}=\int_{\text {skydome }} R(\theta, \phi) \cos \psi_{s} d \Omega
$$

where:

$$
\begin{aligned}
& \psi_{s}=\cos ^{-1}\left(\hat{n}_{s k y} \bullet \hat{n}_{s k y}\right) \\
& \hat{n}_{s k y}=\text { unit vector to point }(\dot{\theta}, \phi) \\
& \hat{n}_{\text {surf }}=\text { outward unit vector normal to surface } \\
& d \Omega=\text { differential solid angle }=\sin \theta d \theta d \phi
\end{aligned}
$$

The pyranometer diffuse irradiance data (for horizontal, shaded vertical, and shaded horizontal) will cross-check the accuracy of the derived diffuse sky irradiance, to define error limits of the method. These data can then be used to document the inaccuracy of various isotropic and anisotropic models inherent in the codes that are used to obtain sky diffuse values. If data can be taken continuously, then averages for all conditions can be calculated to define the average anisotropic effects for this climate and location to be incorporated into code processors. More accuracy would depend on information on cloud type and spatial distribution.

Finally, note that spectra from approximately 0.3 to $3 \mu \mathrm{m}$ are also being studied by others for direct beam and global horizontal fluxes. Spectra are available for selected days, as shown in Figure 6-14 [10].

\subsubsection{Convection Film Coefficients}

To separate and measure the convective and infrared fluxes at wall boundaries, particularly for the glazings, we intend to isolate the convective film with an aluminized mylar covering [20], correcting residual infrared coupling with view factor theory. Infrared fluxes then can be computed subtractively when the foil is removed and compared to the view result theory for methodology checking. The method is outlined in Figure 6-15 and is discussed below. 


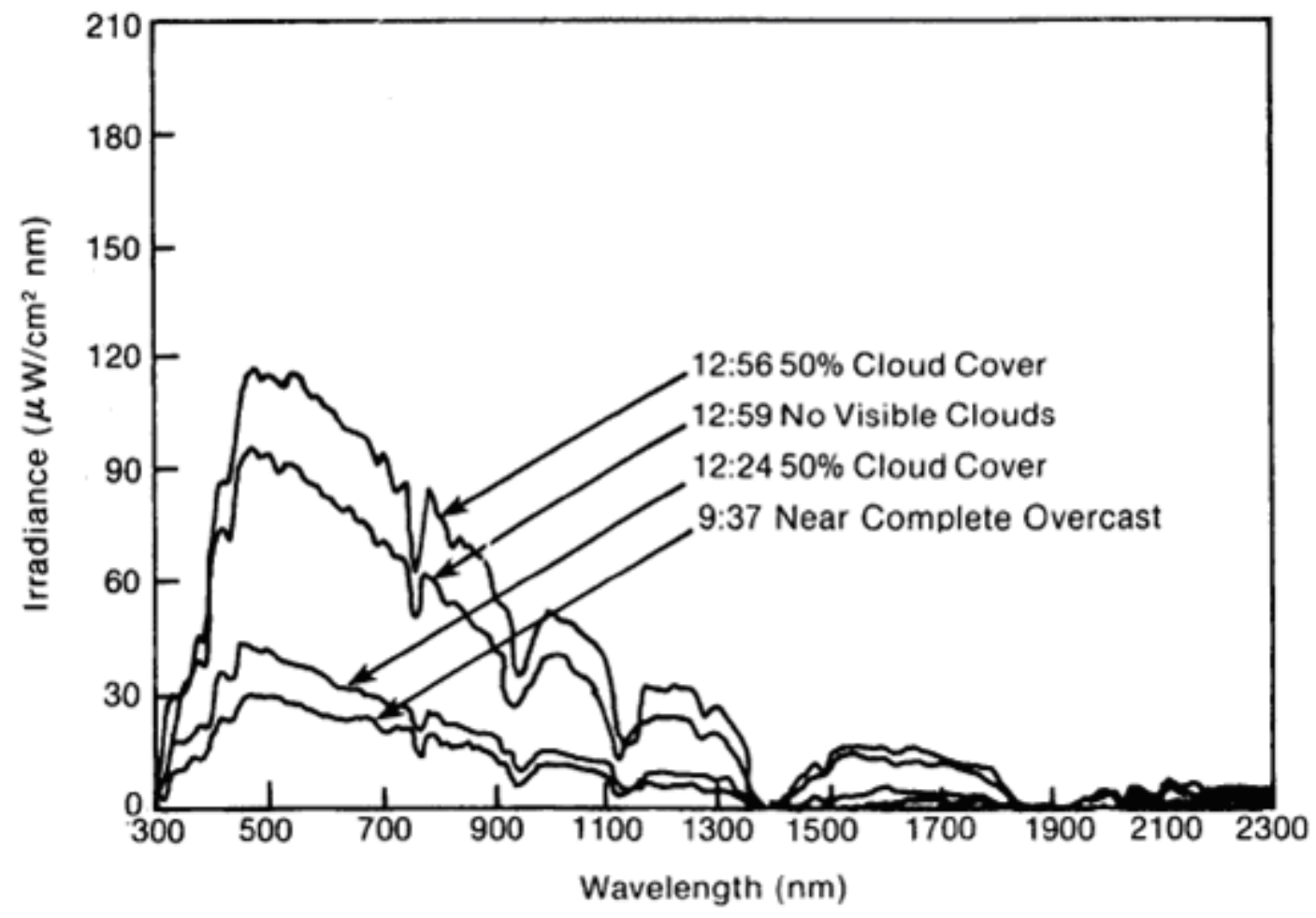

Figure 6-14. Global horizontal spectra

The view factor theory is known to be reliable when surface optical parameters are known and convergence in area subdivision is assured [21]. An equation for correcting infrared effects thus can be written. Consider the inside surface boundary conditions, as shown in Figure 6-15. At the $\mathrm{j}^{\text {th }}$ location where the film coefficient is measured, the local mean radiant temperature $T_{\mathrm{mrt}}$ and mean emissivity $\epsilon_{j}$ can be defined as:

$$
\begin{aligned}
& T_{M R T, j} \equiv \sum_{i=\text { surf }} F_{j i} T_{i}, F_{i i}=0 \\
& \bar{\epsilon}_{j} \equiv \sum_{i=\text { surf }} F_{j i} \in_{i}
\end{aligned}
$$

Where,

$\begin{array}{lll}\mathrm{i} & =\text { all surfaces, } \\ \mathrm{T}_{\mathrm{i}} & =\text { temperature at center of } \mathrm{i}_{\mathrm{th}} \text { surface, and } \\ \mathrm{F}_{\mathrm{ij}} & =\text { view factor. }\end{array}$




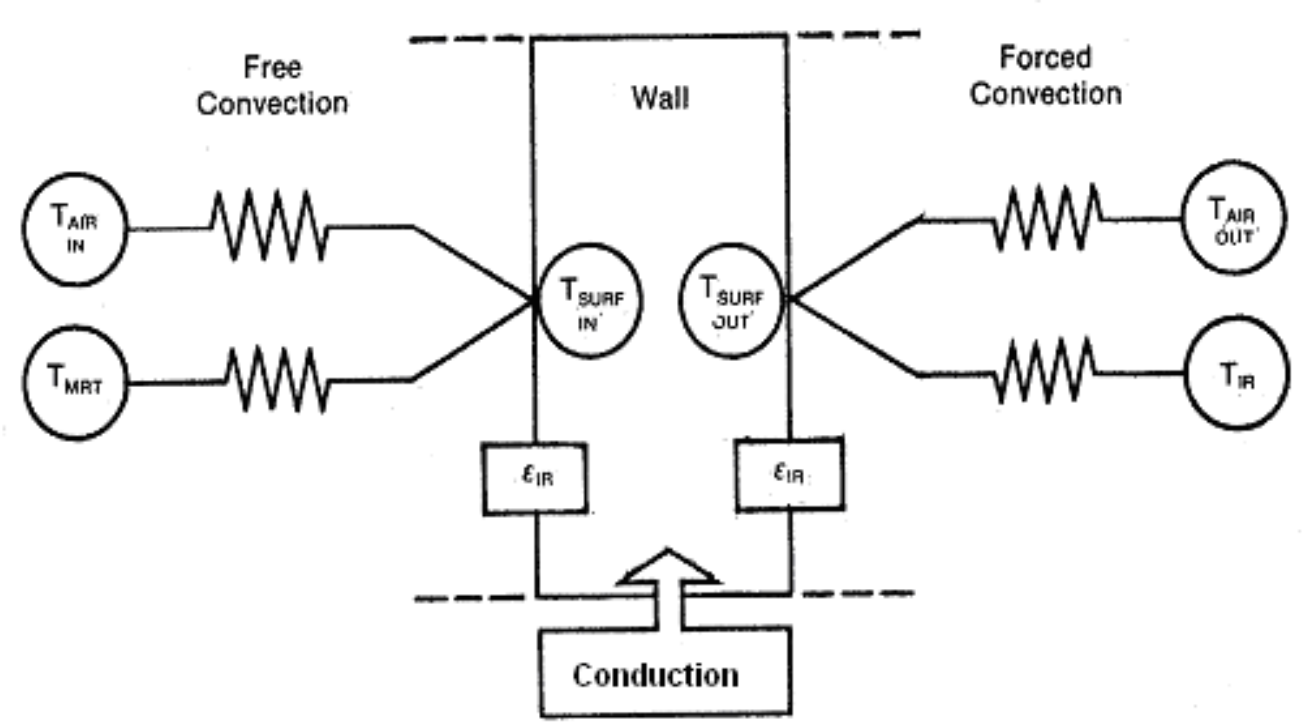

1. Low emissivity foil coating : $\varepsilon<0.05 \rightarrow \dot{Q}_{I R} \cong h_{I R}\left(T_{S U R F}-T_{M R T}\right)$

$$
\rightarrow h_{c v} \cong \frac{Q_{M E A S}-\dot{Q}_{I R}}{T_{S U R F}-T_{A I R}}
$$

2. No coating $: \varepsilon_{I R} \sim 0.95$.

$$
\begin{aligned}
-\dot{Q}_{M E A S} & =h_{C V}\left(\Delta T_{A I R}\right)+h_{I R}\left(\Delta T_{I R}\right) \\
& \rightarrow h_{I R}=\frac{\dot{Q}_{M E A S}-h_{C V} \Delta T_{A I R}}{\Delta T_{I R}}(\text { consistency check }) \\
& \rightarrow h_{C V} \cong \frac{\dot{Q}_{M E A S}-h_{I R}\left(\Delta T_{I R}\right)}{\Delta T_{A I R}}
\end{aligned}
$$

Figure 6-15. Wall boundary conditions

Note that $\mathrm{T}_{\text {mrt,j }}$ will be calculated from measured surface temperatures $\mathrm{T}_{\mathrm{i}}$. Also, local air temperature at $T_{j}$ may differ significantly from the zone average air temperature and, hence, must be measured close to the $\mathrm{j}^{\text {th }}$ location. The result for one surface entirely surrounding another [22] leads to

$$
\begin{aligned}
& \dot{Q}_{I R}=\varepsilon_{j}^{1} \sigma\left(T_{j}^{4}-T_{M R T}^{4}\right)=h_{I R, j}\left(T_{j}-T_{M R T}\right), \\
& h_{I R j}=\varepsilon_{j}^{1} \sigma\left(T_{j}^{2}+T_{M R T}^{2}\right)\left(T_{j}+T_{M R T}\right), \\
& \varepsilon_{j}^{1}=\left(1 / \epsilon_{j}+1 / \bar{\varepsilon}-1\right)^{-1}
\end{aligned}
$$

Since convection exists simultaneously, the measured flux includes both convective and infrared fluxes, $\dot{Q}_{\text {meas }}=\dot{Q}_{\text {conv }}+\dot{Q}_{\text {ir }}$ 
Now consider the $\mathrm{j}_{\text {th }}$ location being covered with low emissivity aluminum foil $(\varepsilon<0.05)$. In this case, $\dot{Q}_{\text {ir }}$ is small and $\dot{Q}_{\text {meas }}$ is nearly all due to convection. Failure to correct for $\dot{Q}_{\text {r }}$ leads to about a $6 \%$ overestimation for $\varepsilon=0.05$ and other reasonable assumptions on the convective term (21]. The same fluxmeter should be used to avoid effects of calibration error.

Now consider the $\mathrm{k}^{\text {th }}$ location nearby at the same orientation and height, but with normal IR emissivity in the 0.9-0.95 range. If the surface temperature difference $\mathrm{T}_{\mathrm{k}}-\mathrm{T}_{\mathrm{j}}$ is small (as for insulated walls away from glazings), then $\mathrm{h}_{\text {conv }, \mathrm{j}}=\mathrm{h}_{\text {conv,k }}$ and $\dot{Q}_{\text {ir }}=\dot{Q}_{\text {meas }}-\dot{Q}_{\text {conv. }}$

Choice of $n$ value is a second order effect. This value can now be compared to the computed enclosure theory result, again cross-checking results to give a measure of the internal consistency of the method and establish confidence levels.

Convective film coefficients will be measured for horizontal, upward, and downward flow conditions. In the test cell this theory is tested more rigorously by examining wall locations both close to and far from glazings, thus having very different view factors to the cold infrared sink, the glazing. Since areas near the glazings also have the largest solar flux, the cell data will indicate whether average irradiances and couplings lead to correct total flux for side walls and floor.

In the case of external surfaces it is inappropriate to repeat this procedure because of the continual wind-driven variation in the film value. This induces dramatic changes in measured surface flux and presents problems in sampling and averaging. Hence, our approach is to determine the external convection film only as an average during the coheating experiment, computing infrared flux average directly and subtracting it from the long-term average flux measured at the interior surface; $\mathrm{h}_{\text {conv }}=\left(\dot{Q}_{\text {meas }}-\dot{Q}_{\text {ir }}\right) / \Delta \mathrm{T}$. To compute the infrared flux, one must know the infrared background temperature. For the horizontal roof, this is the sky temperature as measured. For the vertical walls, this exchange temperature will be computed as a view-factor-weighted average with ground, ambient, and sky temperature, assuming that from horizontal to $\Theta_{\text {cut }}=30 \mathrm{deg}$, ambient $=$ infrared temperature in that direction. Further study is needed to improve tilted surface infrared irradiance from measured $\dot{Q}_{\text {ir,horiz. }}$

\subsubsection{Experiment Planning}

In the case of the cell, experiments are planned that proceed from the simple to the complex, in which additional mechanisms or complexities are introduced one at a time to isolate groups of mechanisms. In addition, the point where a code stops agreeing with data will indicate that the model or solution process for the added mechanisms may cause the error. In the case of the house, each configuration will be studied with all mechanisms present.

Test cell experiments. Test cell experiment plans are given in Table 6-7, divided into heating season and cooling season. The heating season experiments start with conduction experiments, with the sun excluded from the cell interior. This leaves the wall and glazing conduction as the dominant mechanisms. The remaining experiments include solar gain in two zones and conduction. 
The first class of heating season experiments is performed with the glazings entirely blocked with white paint. The first three experiments differ only in the conditions of the internal heat addition. In experiment No. 1, only destratifier power is input, and the zones float mainly in relation to ambient temperature. The damping of the temperature swing and the phase shifts are the key system validation variables. The code predictions should closely follow these data, since errors in mass and load computation will change the amplitude and phase only slightly.

Experiment No. 2 adds a substantial amount of heat to each cell, elevating the average zone temperature $\sim 40^{\circ} \mathrm{C}\left(70^{\circ} \mathrm{F}\right)$ and $\sim 20^{\circ} \mathrm{C}\left(35^{\circ} \mathrm{F}\right)$ above ambient on east and west zones, respectively. Errors in load will be amplified on the zone temperature variable, with $\delta \mathrm{T} \approx(\mathrm{Q} / \mathrm{L})(\delta \mathrm{L} / \mathrm{L})$ where $\delta$ indicates deviation between code and data or actual value. For $\delta \mathrm{L} / \mathrm{L} \approx 10 \%, \delta \mathrm{T} \sim 40^{\circ} \mathrm{C}\left(70^{\circ} \mathrm{F}\right)$ (east) or $2^{\circ} \mathrm{C}\left(4^{\circ} \mathrm{F}\right)$ (west). The third experiment constrains the temperature of each zone to be $\sim 24^{\circ} \mathrm{C}\left(75^{\circ} \mathrm{F}\right)$. The auxiliary used is the key system variable, with $\delta \mathrm{Q} \sim \delta \mathrm{L} \times \bar{\Delta} \bar{T}$ where the bar indicates averaging. A $10 \%$ load error produces a $10 \%$ auxiliary error. Coheating data will be extracted from this experiment.

At this stage when the codes are compared to the available data, conduction through the walls and the glazing will be the dominant mechanism. Secondary mechanisms are infiltration, external surface solar loading, and external infrared coupling. If there is a significant discrepancy between code and data, it must be investigated and corrected before the solar mechanism is added. The only significant problem might be the U-value assumed for the glazings, which may need adjustment as indicated by fluxmeter and temperature data on glazings. The codes simulate the opaque wall conduction problem quite well (see Section 5.0 and Appendix A). Therefore, the wall conduction problem in the codes, in essence, is normalized here, correctly fitting the conduction problems before the solar mechanism is added.

The remaining experiments involve strong solar collection through the glazings, different control strategies, and zone coupling. The first sun experiment (No. 4) is the same as conduction experiment No. 3 except for the glazings, and it therefore focuses on the solar driving effects. The second sun experiment (No. 5) differs from the first in that the west zone is thermostated and includes setback at night, while the east zone is not thermostated. Hence, the focus is on the effects of the thermostat strategy change. In both experiments, there will be considerable interzonal flux, with more in No. 5 than in No. 4. Experiment No. 5 is purposefully like a living space (west zone-low mass, reduced glazing, and small temperature swing) coupled by conduction to a sun space (east zone - high mass, high sun input, and large temperature swing). The remaining experiments differ from case No. 5 in one aspect.

First, interzonal coupling can be inhibited with addition of insulation (No. 6), or it can be maximized by sealing the west glazing entirely (No. 7). If experiment No. 5 is successful with the code comparison, No. 6 can be eliminated. However, if there is confusion about sources of the errors, then No. 6 will be done because it presents a simpler case than No. 5 .

If time or funding permits, additional heating experiments will include night insulation on the high-mass glazing (No. 8) to provide high-quality data on this important feature of passive systems. Thermax boards will be manually raised and lowered and will be applied externally with special attention to edge sealing. Finally, free-floating data will be taken. Low priority is 
attached to free-floating data because several existing test cells already provide this kind of data [18].

The cooling season experiments, like those for heating, emphasize auxiliary mechanisms. An air conditioner will be used to maintain control on the west zone, in the sense $T_{\text {west }} \leq \mathrm{T}_{\text {set. }}$ Some free-float data will also be obtained (experiment No. 1) to test temperature swing calculations in the simplest case where no auxiliary mechanisms are present.

As in the heating case, variations on a base case are used to isolate specific mechanisms for achieving desired cooling load reduction. The base case is experiment No. 2, wherein solar loading is countered only by operation of an air conditioner in the west zone. Next, night ventilation can be tested. The control strategy, for example, could be to ventilate at $\sim 15 \mathrm{ACH}$ when $\mathrm{T}_{\text {out }}<\mathrm{T}_{\text {zone }}-2^{\circ} \mathrm{C}\left(5^{\circ} \mathrm{F}\right)$. Second, code calculations for shading the glazings can be tested. An overhang $\sim 2 \mathrm{~m}(6 \mathrm{ft})$ wide and $\sim 3 \mathrm{~m}(10 \mathrm{ft})$ long will extend from the roof over the glazings, resulting in considerable reduction of the zone loading. Ventilation and shading can be tested together as a reasonable approach to minimizing or eliminating the cooling load in the west zone.

Additional data that must be acquired include time intervals with the tracking pyrheliometer behind the glazing. Starting with experiment No. 4, the pyrheliometer will be run for the first several days of each experiment. Using this approach, both clear and cloudy days will be measured at a variety of sun angles. Cell albedo will be measured twice between runs.

House experiments. House heating season experiment plans are given in Table 6-8. The cases to be studied during the heating seasons are: No. 1, the base house (house before retrofits), No. 2, the conservation house (insulated zone skin as reasonable, installed storm windows, tight structure, etc.), and No. 3, the house with an added solar gain component. In each configuration, an experiment is planned in which the zones are uncoupled except for interior wall conduction, and zone temperatures are constrained as $\mathrm{T}_{\text {zonej }}>\mathrm{T}_{\text {set }}$ with $\mathrm{T}_{\text {set }}$ the same for all zones. This run will be used to extract coheating wall property data in addition to providing validation data where multi-zone effects are simplified. Solar heat, skin conduction, and infiltration will be the dominant mechanisms in the building energy balance.

Additional two-week runs will be made with a different zone control that leads to much larger interzonal thermal coupling. In the base case house, a free float run should show large temperature swings in the south zones and considerable interzonal wall conduction between north and south zones.

In the conservation and solar houses, runs will be made with the doors open, once we can measure interzonal flow via a multiple tracer gas system presently under conceptual development. The interzonal advection problem has been reserved for special attention. Convective flow is sufficiently complex that it should be measured directly and studied in a building for which accurate data on all other mechanisms have already been obtained.

Plans for cooling experiments are not listed in Table 6-8, but include night ventilation of the structure, window shading, and an evaporative cooler. Note that this work will be achieved in the future. 
Table 6-7. Cell Experiment Schedule

\begin{tabular}{|c|c|c|c|c|c|}
\hline & Experiment & Duration & Zone Controls & Sun Condition & Description and Purpose \\
\hline \multicolumn{6}{|c|}{ Heating Season Experiments: } \\
\hline 1 & No sun + floating & 1 week & None & Blocked in both zones & $\begin{array}{l}\text { Simplest possible dynamic conduction loads problem; } \\
\text { no interior sunlight or temperature constraints }\end{array}$ \\
\hline 2 & No sun + constant input & 2 weeks & $\begin{array}{l}250-W(700-W) \text { heaters on } \\
\text { continuously in west (east) } \\
\text { zone }\end{array}$ & - & $\begin{array}{l}\text { Constant heat addition to the zone air only, simplest } \\
\text { energy input }\end{array}$ \\
\hline 3 & No sun + controlled & $\begin{array}{l}2-3 \text { weeks } \\
\text { (weather } \\
\text { dependent) }\end{array}$ & Both sides $T_{\text {zone }}(T) \geq T_{\text {set }}$ & Blocked in both zones & $\begin{array}{l}\text { Temperature-controlled case, simplest loads; coheating } \\
\text { data taken out during steadiest period(s) }\end{array}$ \\
\hline 4 & Sun + controlled & 2 weeks & Both sides $T_{\text {zone }}(T) \geq T_{\text {set }}$ & $\begin{array}{l}\text { Sun admitted to both } \\
\text { zones }\end{array}$ & $\begin{array}{l}\text { Only change from No. } 2 \text { is sun with interior: isolate } \\
\text { interior solar effects }\end{array}$ \\
\hline 5 & Sun + partial control & 2 weeks & $\begin{array}{l}\text { Free-float, high-mass; } \\
\text { constrained low-mass only, } \\
\text { with night setback }\end{array}$ & $\begin{array}{l}\text { Sun admitted to both } \\
\text { zones }\end{array}$ & $\begin{array}{l}\text { Only change from No. } 3 \text { is removal of constraint control } \\
\text { in high-mass zone, making system like realistic } \\
\text { sunspace + controlled living space. Will obtain large } \\
\text { interzonal flux; test night setback effects. }\end{array}$ \\
\hline 6 & $\begin{array}{l}\text { Sun + partial control }+ \\
\text { inhibited interzone } \\
\text { coupling }\end{array}$ & 2 weeks & $\begin{array}{l}\text { Free-float, high-mass; control } \\
\text { in constrained low-mass only, } \\
\text { with night setback }\end{array}$ & $\begin{array}{l}\text { Sun admitted to both } \\
\text { zones }\end{array}$ & $\begin{array}{l}\text { Only change from No. } 5 \text { is order of magnitude increase } \\
\text { in partition wall } R \text { value, helping isolate effects of } \\
\text { interzonal flux mechanism by nearly eliminating it here }\end{array}$ \\
\hline \multicolumn{6}{|c|}{ As time permits: } \\
\hline 7 & $\begin{array}{l}\text { Partial sun + partial } \\
\text { control }\end{array}$ & 2 weeks & $\begin{array}{l}\text { Free-float, high-mass; control } \\
\text { in constrained low-mass only, } \\
\text { with night setback }\end{array}$ & $\begin{array}{l}\text { Block sun on low-mass } \\
\text { only }\end{array}$ & $\begin{array}{l}\text { Only change from No. } 5 \text { is sun blockage on west; will } \\
\text { get maximum interzonal coupling }\end{array}$ \\
\hline 8 & $\begin{array}{l}\text { Sun + partial control + } \\
\text { night insulation }\end{array}$ & $\begin{array}{l}2 \text { weeks } \\
\text { (time } \\
\text { permitting) }\end{array}$ & $\begin{array}{l}\text { Free-float, high-mass; control } \\
\text { in constrained low-mass only, } \\
\text { with night setback }\end{array}$ & Sun to both sides & $\begin{array}{l}\text { Same as No. } 5 \text { except high-mass glazing annually } \\
\text { insulated at night with external R-12 board }\end{array}$ \\
\hline 9 & Sun + floating & 2 weeks & None & Sun to both sides & Same as No. 5 except free-floating zone condition \\
\hline \multicolumn{6}{|c|}{ Cooling Season Experiments: } \\
\hline 1 & Overheating base case & $\sim 2$ weeks & None & Sun admitted with zones & $\begin{array}{l}\text { Testing overheating and temperature swing } \\
\text { calculations when inputs exceed load at comfort and no } \\
\text { control is used }\end{array}$ \\
\hline 2 & Cooling load base case & $\sim 3$ weeks & Control $\mathrm{T}_{w} \leq \mathrm{t}_{\text {set }}$ & $\begin{array}{l}\text { Sun admitted to both } \\
\text { zones }\end{array}$ & $\begin{array}{l}\text { Cooling load in west zone during overheating period } \\
\text { including conduction from overheated east zone }\end{array}$ \\
\hline 3 & Night ventilation & $4-6$ weeks & $\begin{array}{l}\text { Ventilation when } \mathrm{T}_{\text {out }}<\mathrm{T}_{\text {zone }}- \\
\sim 2^{\circ} \mathrm{C}\left(5^{\circ} \mathrm{F}\right) \text { and } \mathrm{T}_{\text {west }} \leq \mathrm{T}_{\text {set }}\end{array}$ & $\begin{array}{l}\text { Sun admitted to both } \\
\text { zones }\end{array}$ & $\begin{array}{l}\text { Determining effect of ventilation in the high-mass and } \\
\text { low-mass cells, at } 15 \mathrm{ACH}\end{array}$ \\
\hline 4 & Shading & 3 weeks & - & $\begin{array}{l}\text { Substantial overhang } \\
\text { introduced over both } \\
\text { glazings }\end{array}$ & $\begin{array}{l}\text { Determining cooling load when there is significant } \\
\text { shading }\end{array}$ \\
\hline 5 & Shading + ventilation & $\begin{array}{l}\sim 3 \text { weeks } \\
\text { load }\end{array}$ & Control $\mathrm{T}_{\text {west }} \leq \mathrm{T}_{\text {set }}$ & Substantial shading & Combined mechanism approach to eliminating cooling \\
\hline
\end{tabular}


Table 6-8. Validation Test House Experiments for the Heating Season

\begin{tabular}{|l|l|l|}
\hline \multicolumn{1}{|c|}{ Experiment } & \multicolumn{1}{|c|}{ Description } & \multicolumn{1}{c|}{ Characteristics } \\
\hline No. 1: Base House & $\begin{array}{l}\text { Loads dominated with high } \\
\text { infiltration; moderate direct gain, } \\
\text { low mass. High energy } \\
\text { consumption. }\end{array}$ & $\begin{array}{l}\text { Free float: large temperature } \\
\text { swings, large interzonal } \\
\text { conduction flux } \\
\text { Controlled temperature: } \\
\text { coheating data and simplest } \\
\text { system }\end{array}$ \\
\hline No. 2: Conservation House & $\begin{array}{l}\text { Modestly tight house, reduced } \\
\text { energy consumption, moderate } \\
\text { direct gain, low mass }\end{array}$ & $\begin{array}{l}\text { Controlled temperature: } \\
\text { coheating data and simplest } \\
\text { system } \\
\text { Varied control strategy }\end{array}$ \\
\hline No. 3: Solar House & $\begin{array}{l}\text { Moderately tight house, } \\
\text { moderate direct gain, medium- } \\
\text { sized passive solar system, low } \\
\text { energy use }\end{array}$ & $\begin{array}{l}\text { Controlled temperature: } \\
\text { coheating data and simplest } \\
\text { system } \\
\text { Varied control strategy }\end{array}$ \\
\hline
\end{tabular}

\subsection{Data Processing}

In this section, the hardware and software used to process the data are described briefly.

\subsubsection{Data Hardware}

The data acquisition system (DAS) is shown in Figure 6-16. Data flow is controlled by a multitask LSI 11/23 microprocessor (128K bytes), with inputs and outputs routed through CAMAC crates. The microprocessor has disk storage of $\sim 5$ megabytes to store processing software and keep data files temporarily. Disk storage retains a week's time-averaged data.

\subsubsection{Inputs}

Sensor inputs include thermocouples, fluxmeters, watt meters, and pyranometers. Except for fast-sampling channels (auxiliary power and pyranometers), signals are sampled by a Fluke 2240B, multiplexing signals through Fluke 2204A remote scanner units behind the cell and in the utility room in the house. Temperature $\left(0^{\circ} \mathrm{C}\right.$ and $38^{\circ} \mathrm{C}\left[32^{\circ}\right.$ and $\left.\left.10^{\circ} \mathrm{F}\right]\right)$ and millivolt $(5.0 \mathrm{mV})$ calibration signals are also input each scan at 5-min intervals. The temperatures are converted to ${ }^{\circ} \mathrm{F}$ in the Fluke 2240B.

The digitized signals are then routed to the LSI/11 through CAMAC RS232C serial data ports. The Fluke loggers are controlled for data flow by the LSI/11 through a CAMAC interface board. The LSI reads 2000 Fluke channels every five minutes, and performs hourly time averages.

Fast scan data are brought directly to multiplexers in the CANAC crates, digitized once every 10 seconds and routed to the LSI/II. Hourly averages are performed and stored on the disk. Other front end data loggers can also be used to digitize signals. The site possesses several Auto Data 10/10, Digi3, and Kaye data loggers that can communicate through RS232C ports. All together, the field site can process nearly 3000 channels of data. At present, approximately 2100 channels are being read, resulting in $\sim 1.5$ megasamples per day. 


\subsubsection{Outputs}

Researchers at the site can examine any portion of the data stored on disk. RS232C data ports link the LSI to several terminals for screen monitoring, printing, or plotting on a Hewlett Packard Plotter. At present, once each week the data are recorded on 9-track tape for processing on SERI's mainframe computer, a CYBER 170/720. The LSI/11 can also control experimental devices through CAMAC on/off switches. Movable glass louvers, bead-board, and aluminized plastic film insulation devices are controlled with other test cell data discussed in Section 6.4. The researchers will control the heaters in the cell and the house and use automatic lowering of thermostats at night.

As shown in Figure 6-16, a backup data output path is available if the LSI/CAMAC system is inoperative. Front-end data loggers can use various recording media that can be hand-carried to the CYBER. Sample rates and channel volume are curtailed in this backup mode.

\subsubsection{Data Software}

The bulk of the data processing and analysis will be done on the mainframe computers.

\subsubsection{Data Processing}

Software is being developed to perform the following data processing tasks:

- Convert differential thermocouple voltages to absolute temperature difference.

- Include temperature dependence in the fluxmeter and C·15/6 pyranometer outputs, and convert to physical units.

- Check calibration channels and filter data for unreasonable values.

- Compute sun angles and subtract beam components from global pyranometer reading.

\subsubsection{Data Analysis}

Data analysis on the SERI CYBER will be performed with the user code SPSS, a welldocumented data handling and statistics package [23]. SPSS will be used to compute the following:

- Time averaging for the backup data flow option and for the minute-average environmental data from the resource assessment group [6]

- File merging and arranging

- Deriving weighted averages from temperature and flux data at different wall locations, including average surface temperatures, flux. and $\mathrm{Q}_{\text {store }}$

- Computing the code-data comparison variables of Table 6-4 for code-data comparisons 


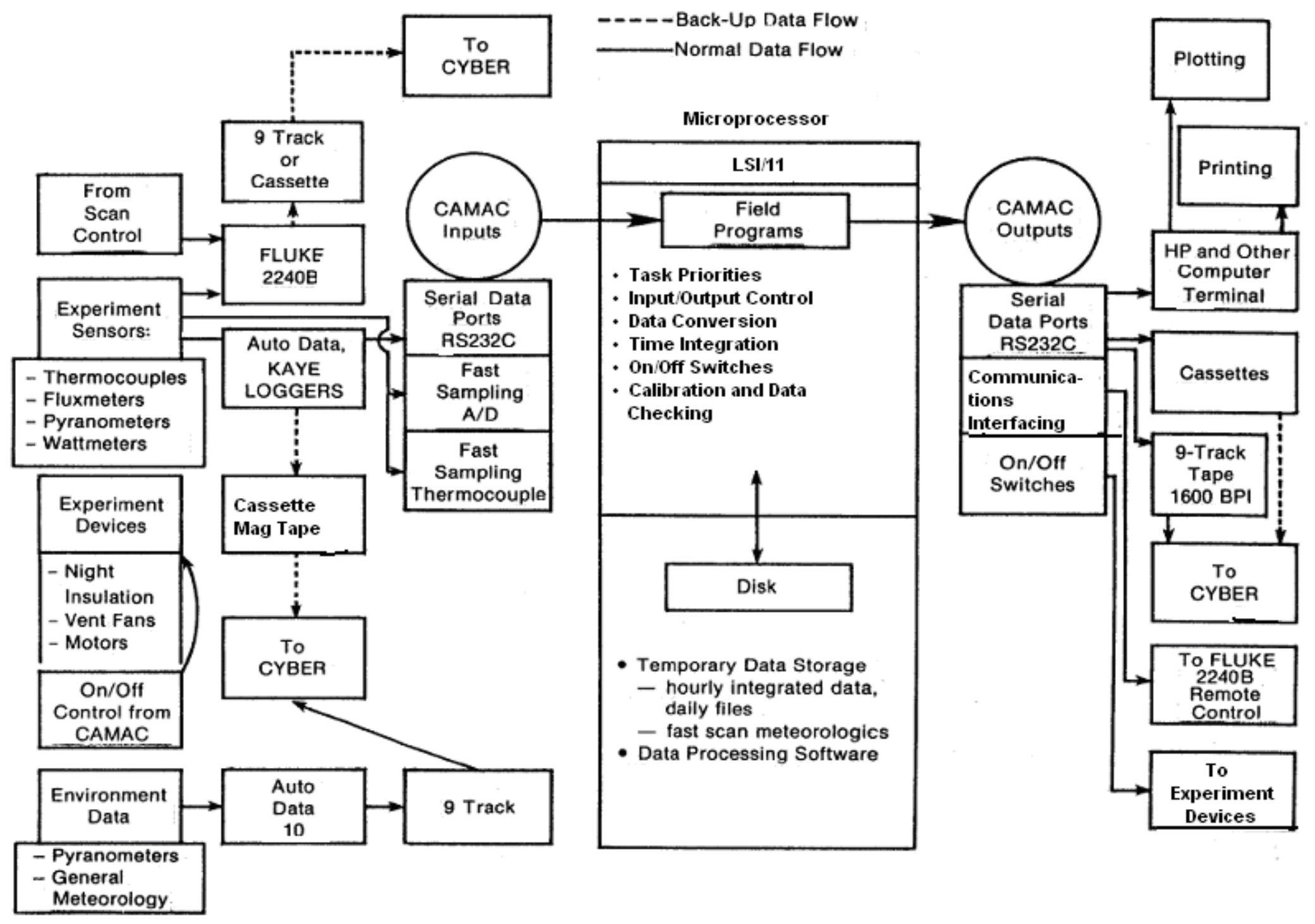

Figure 6-16. Data flow chart 
- Correlating variables over short and long duration: bivariate or multiple; linear or nonlinear

- Extracting best fit glazing input parameters from transmission data and other similar data and parameter least squares analyses.

Raw and processed data files will be maintained so that, in addition to selected published data, researchers can access data from the validation experiments or any of the other validation data sources in Section 6.4. The system will be able to: (1) display and print tabular values at the terminal; (2) plot specified channels for specified intervals, either on a line printer for many plots or on system plotters for low-volume plotting; (3) produce line printer hard copy of values; and (4) produce magnetic tape copies for non-SERI validations. These specific tasks will be incorporated into interactive procedure, files that minimize the keystrokes required.

Four types of files will be maintained on the CYBER:

- Raw data files (archived on disk pack and on magnetic tape)

- Summary level files, consisting of environmental data and all system-level raw data and related computed quantities (archived on disk pack)

- Mechanism level data files, containing raw and computed variables relating to solar processing and other mechanisms (archived on magnetic tape)

- Message and description files that document experiment setup, data anomalies, and control actions.

\subsection{Other Sources of Validation Data at SERI}

The test cell is specifically dedicated to providing code validation sources. However, data from several other projects can be used for code validation as a secondary purpose. These data sources and the intended data use are briefly described in this section.

\subsubsection{Performance Test Group Cells, Nos. 1 to 4}

Four test cells, identical in framing and glazing to the validation group cell, have been installed and instrumented by SERI's performance test group [24]. These cells have about half of the insulation in the building skin as that of the validation test cell. Each cell is divided into two zones and contains an identical water or masonry mass wall. The zones are separated by an insulated partition that minimizes the zone coupling coefficient. In each cell, one zone consists of a standard mass wall. The other zone contains either nighttime insulation with foils and boards, or front and back louvers or removable film barriers to change zone-wall coupling. In two of the cells, the mass walls are immediately behind the glazings. In the other two cells, the mass walls are about $2 \mathrm{~m}(5 \mathrm{ft})$ behind the glazing to allow variable controls on the front surface.

Some zones that contain the control features will not be useful for validation, because the codes are not capable of incorporating such features. These data, however, may be useful for future code developments. The codes can simulate some standard night insulation on mass wall surfaces. In addition, the uncontrolled zones are within the codes' capabilities. Hence, at least four of these zones will provide data directly useful for code validation. 
The zones are generally instrumented for air temperature, wall surface temperature, mass wall temperature, and mass wall flux. Overall load coefficient has been measured for each zone in a coheating experiment.

Indirect as opposed to direct charging of mass walls can be studied from these data. For both the water and masonry wall standard case, the zones are identical with walls both next to the

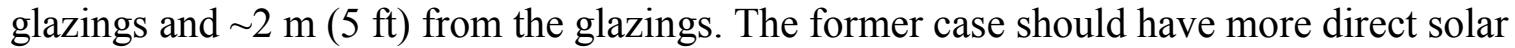
radiation. Hence, comparisons will yield an efficiency decrease because of a decrease in the direct charging. This comparison is best made in fall or spring when sun angles are still relatively high.

\subsubsection{Selective Surface Test Box}

As shown in Figure 6-17, a $1 \times 1 \times 1 \times 1-\mathrm{m}(3 \times 3 \times 3 \times 3$-ft $)$ test box has been built and instrumented to assess selective surface performance directly. The effect of delamination of foil with the masonry is emphasized. Four Trombe wall segments are to be compared and consist of: (1) a standard black-painted wall; (2) a wall with selective surface foil; (3) a wall with the foil set back $3 \mathrm{~mm}$ (0.13 in.) from surface; and (4) the same wall as (3), but with 6-mm (0.25-in.) spacing. The box is double-glazed, and all wall units are thermally isolated, constrained to onedimensional flow conditions, and identically shaded. The temperature of the zone behind the 3.5$\mathrm{m}$ (12-ft) thick mass units is constrained. Flux from the back of each unit is measured directly, and interval integrations of this flux are a suitable direct measure of performance comparison. In addition, front and back surface, mass midpoint, glazing gap, and inner surface temperatures are measured for each wall unit to provide insight into some of the thermal dynamics. 


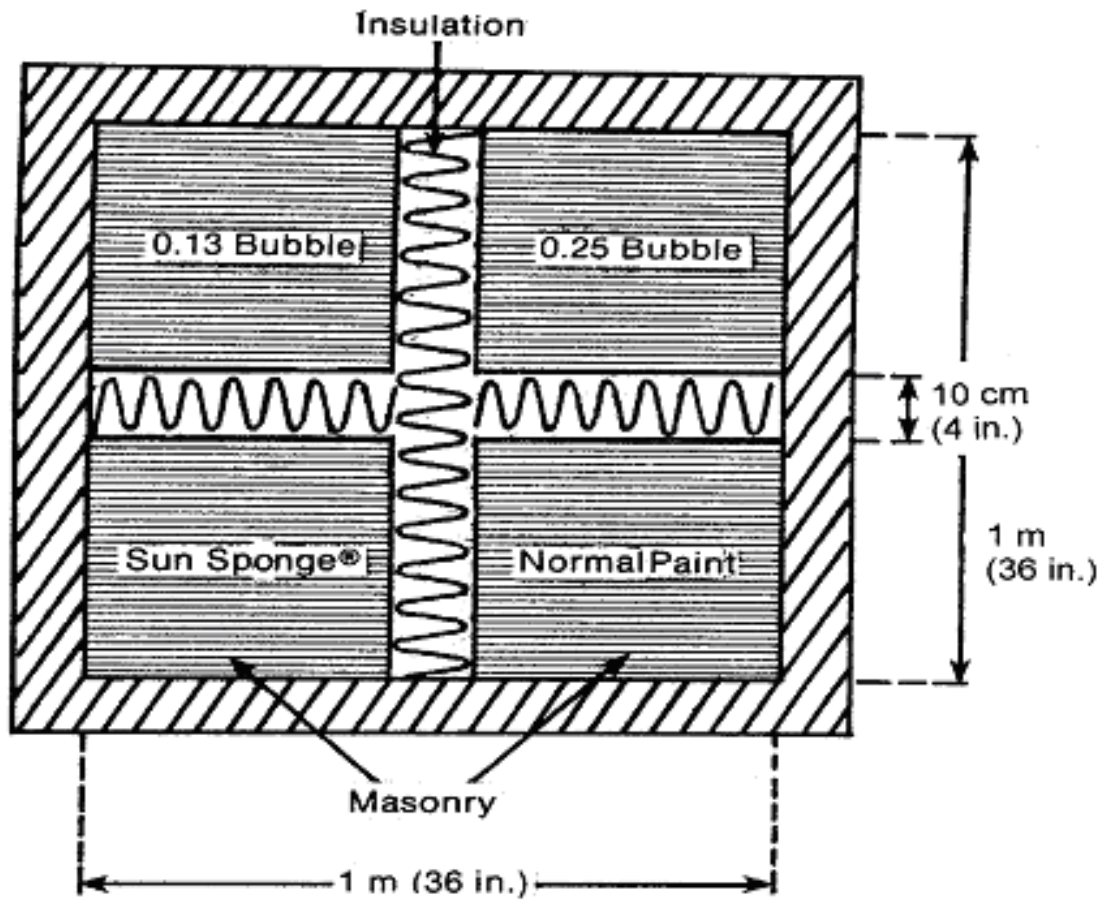

Front View

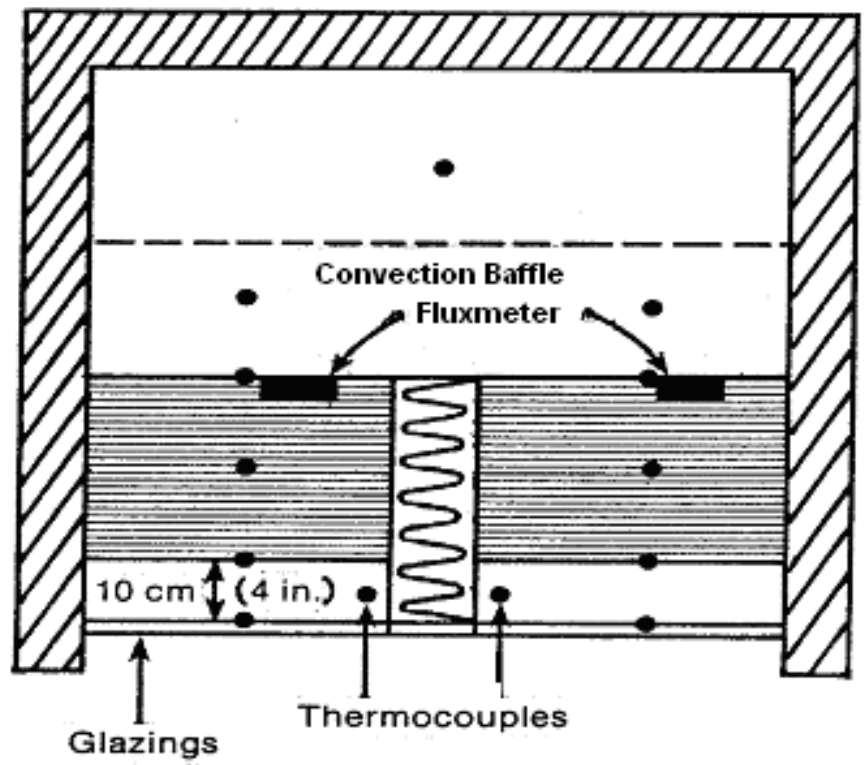

Top View

Figure 6-17. Selective surface test box 


\subsection{Nomenclature}

$\begin{array}{ll}\text { Symbols } & \\ \text { A } & \text { area, or constant } \\ \text { c } & \text { specific heat } \\ \text { C } & \text { heat capacity or tracer gas concentration } \\ \text { E } & \text { designed error or effective emissivity } \\ \text { f } & \text { weighting factor } \\ \text { F } & \text { view factor } \\ \text { G } & \text { global irradiance (2 } \pi \text { steradians) } \\ \text { h } & \text { film coefficient, linearized } \\ \text { H } & \text { floor-to-ceiling height } \\ \text { I } & \text { solar beam, normal incidence } \\ \text { K } & \text { conductivity } \\ \text { L } & \text { load coefficient, or length } \\ \text { n } & \text { index of refraction for glazings } \\ \mathrm{N} & \text { number of samples per hour } \\ \mathrm{Q} & \text { energy } \\ \dot{Q} & \text { power (dQ/dt) } \\ \mathrm{R} & \text { sky radiance } \\ \mathrm{S} & \text { signal } \\ \mathrm{t} & \text { time } \\ \mathrm{T} & \text { temperature } \\ \mathrm{U} & \mathrm{U} \text {-value, steady-state } \\ \dot{V} & \text { volume flow rate } \\ \omega & \text { angular frequency } \\ \mathrm{X} & \text { energy-related variables } \\ \mathrm{z} & \text { zone solar back-loss coefficient } \\ \alpha & \text { opaque surface absorptivity, or sun altitude angle } \\ \Delta, \delta & \text { difference or deviation } \\ \varepsilon & \text { surface infrared emissivity } \\ \theta, \varphi & \text { spherical polar coordinate angles } \\ \eta & \text { constant efficiency, }<1 \\ \kappa & \text { extinction coefficient for glazings or exponent } \\ \lambda & \text { eigenvalues } \\ \rho & \text { mass density } \\ \sigma & \text { Stefan-Boltzman constant, or standard deviation } \\ \tau & \text { transmission of glazings, or time constant } \\ \psi & \text { incident angle } \\ \varphi & \text { sun azimuth angle, or phase shift in radians } \\ \Omega & \text { solid angle, sin d } \theta \text { d } \theta \text { d } \varphi \\ & \end{array}$




$\begin{array}{ll}\text { Subscripts } & \text { and Superscripts } \\ \text { air } & \text { ambient air } \\ \text { amb } & \text { ambient temperature } \\ \text { baf } & \text { baffle } \\ \text { beam } & \text { beam component } \\ \text { cond } & \text { conduction } \\ \text { comp } & \text { comparison } \\ \text { conv } & \text { convection } \\ \text { cut } & \text { cutoff value } \\ \text { dif } & \text { diffuse from sky component } \\ \text { eff } & \text { effective } \\ \text { fm } & \text { fluxmeter } \\ \text { glaz } & \text { glazing air gap } \\ \text { gr } & \text { ground diffuse component } \\ \text { hi } & \text { high mass } \\ \text { hor } & \text { horizontal } \\ \text { i } & \text { wall mass layers, all surfaces } \\ \text { in } & \text { interior } \\ \text { inc } & \text { incident } \\ \text { inf } & \text { infiltration } \\ \text { ir } & \text { infrared } \\ \text { j } & \text { zone } \\ \text { meas } & \text { solar energy measured } \\ \text { mrt } & \text { mean radiant temperature } \\ \text { nb } & \text { normal beam } \\ \text { N.S.E.W. } & \text { direction } \\ \text { out } & \text { outside or ambient } \\ \text { rms } & \text { root mean square } \\ \text { set } & \text { set point } \\ \text { sh } & \text { shielded with baffle } \\ \text { skin } & \text { walls } \\ \text { sky } & \text { sky temperature } \\ \text { so } & \text { solar } \\ \text { stored } & \text { energy stored } \\ \text { surf } & \text { surface } \\ \text { - } & \text { time average } \\ \text { tran } & \text { time differentiation } \\ \text { vert } & \text { transmission } \\ \text { wind } & \text { vertical } \\ \text { zone } & \text { thermal zone in building } \\ & \end{array}$




\subsection{References}

1. Burch, J. 1980. Analytic Validation for Transfer Mechanisms in Passive Simulation Codes, unpublished. Golden, CO: Solar Energy Research Institute.

2. Diamond, S. et a1. 1981. DOE-2 Verification Project: Phase I: Interim Report. LA-8295MS. Washington, DC: U.S. Department of Energy.

3. Myers, J. 1981. Private communication, Lakewood, CO: Valley Laboratory.

4. Shadow Band Instruction Manual, LICOR 810S-22, 1981. Lincoln, NE: LICOR, Inc.,

5. Flowers, E. 1979. Comparison of Solar Radiation Sensors from Various Manufacturers. Boulder, CO: NOAA, ERL.

6. Stoffel, T. et al. 1981. Personal communication. Golden, CO: Solar Energy Research Institute.

7. Cooper, P.I. et al.1981. "A Method of Measuring Sky Temperature," Solar Energy vol. 26, p. 153.P Parkville, Victoria, Australia: Pergamon Press.

8. Standard Practice for Measuring Air Leakage Rate by the Tracer Gas Dilution Method. 1980. E 741-80, ANSI. Philadelphia, PA: ASTM.

9. Cannon, T. and Dwyer, L. 1981. "An All-Sky, Video-Based Luminance Mapper for Daylighting Research,” Proc. 6th Nat. Pas. Conf., Portland, OR.

10. Bird, R. et al. Solar Spectrum Measurements in the Terrestrial Environment. To be published app. Oct. 1982.

11. Burch, J., Seman, J., Wortman, D., and Judkoff, R. Site Handbook for the SERI Test House, SERI Report to be published.

12. Burch, J., Seman, J., Wortman, D., and Judkoff, R. Site Handbook for the SERI Validation Test Cell, SERI Report to be published.

13. Sears, B. et al. Personal communication. Golden, CO: SERI, Buildings Systems Branch.

14. Users Manual: MIDAS II. 1976. Martin Marietta Corp., M-76-2.

15. Hunn, B. Personal Communication. Los Alamos, NM: Los Alamos Scientific Laboratory.

16. "Standard Test Method for Thermal Conductance and Transmittance of Builtup Sections by Means of the Guarded Hot Box." 1973. ASTM Standard C236-66. Philadelphia, PA: ANSI.

17. Sherman, M.H. et al. 1980. Measurement of Infiltration Using Fan Pressurization and Weather Data. Berkeley, CA: Lawrence Laboratories.

18. Andersson, B. et al. Verification of BLAST by Comparison with Direct Gain Test Cell Measurement, LBL-I0619, Berkeley, CA: Lawrence Laboratories.

19. LeBaron, B. et al. 1980. "Corrections for Diffuse Irrad1 ance Measured with Shadowbands," Solar Engineering, vol. 25; p. 1.

20. Winn, C.B. et al. 1981. Continued Studies of Passive SHAC Systems in the REPEAT Facility. Fort Collins, CO: Colorado State University. 
21. Howell, J. and Siegel, R. 1969. Thermal Radiation Heat Transfer, Vol. I-III, NASA-SP164. Houston, TX: National Aeronautical and Space Administration.

22. Chapman, A.J. 1976. Heat Transfer, 3rd Ed. New York: McGraw Hill.

23. Nie, N. et al. 1975. SPSS. A Statistical Package for the Social Sciences. New York: McGraw Hill.

24. Ortega, K. and Connolly, M. 1982. Personal communication, Golden., CO: Solar Energy Research Institute. 


\subsection{Program Descriptions and Comparisons}

\subsection{Introduction}

This section describes and compares the four building energy analysis codes: BLAST 3.0 [1], DEROB 4 [2]. DOE 2.1 [3]. and SUNCAT 2.4 [4]. Section 7.2 briefly compares the four codes and has a table that summarizes the codes' capabilities. Sections 7.3 through 7.6 describe each code individually. We compare and describe the areas of user interface, solution technique, radiation modeling, convection and conduction modeling, and systems and schedules. Although we recognize that each of the codes may have undocumented capabilities, only those capabilities in the code documentation or available to the user on a default basis are described here.

\subsection{Program Comparisons}

BLAST 3.0, DEROB 4, DOE 2.1, and SUNCAT 2.4 are designed to assess the thermal response and energy use of buildings. All are suitable for residential buildings, but BLAST 3.0 and DOE 2.1 are better suited for commercial buildings than SUNCAT 2.4 and DEROB 4 . All compute monthly and yearly thermal loads and hourly temperature variations. Additionally, DEROB 4 can simulate the effects of finite-sized heating and cooling equipment; BLAST 3.0 and DOE 2.1 can simulate the performance of that equipment. BLAST 3.0 and DOE 2.1 include calculations of the life-cycle cost of a building; DEROB 4 and SUNCAT 2.4 do not. All four codes simulate passive solar designs, but DEROB 4 and BLAST 3.0 contain more rigorous modeling of the mechanisms important in such designs. Table 7-1 compares the capabilities of these four codes.

\subsubsection{User Interface}

The user interfaces for BLAST 3.0 and DOE 2.1 differ from those for DEROB 4 and SUNCAT 2.4. BLAST 3.0 and DOE 2.1 use building description languages that have very flexible formats. These languages make input easier for the experienced user, but may be more difficult for the novice. SUNCAT 2.4 has a fixed input structure that is cumbersome for the experienced user but leads the novice toward an acceptable building description. Additionally, the limited scope of SUNCAT 2.4 makes the necessary inputs easier to learn. The building geometry description in DEROB 4 is difficult to prepare, even for an experienced user. Only DEROB 4 requires that input data be placed in the proper columns to be interpreted correctly. The documentation for BLAST 3.0 and DOE 2.1 provides examples of how a user should prepare the input. BLAST 3.0, DOE 2.1, and DEROB 4 contain material property libraries that ease the burden of collecting input data.

SUNCAT 2.4 executes interactively, while the other codes execute in the batch mode. If the interactive and batch modes cost the same to use on a computer system, then SUNCAT 2.4, BLAST 3.0, and DOE 2. 1 require nearly the same resources to simulate a single zone direct gain building for one year. DEROB 4 requires substantially more resources to perform the same simulation.

BLAST 3.0 and DOE 2.1 have the most extensive and flexible output capabilities. DOE 2.1 and DEROB 4 allow the user to observe temperatures and heat flow rates on a surface-by-surface basis. All four codes are capable of hourly output and provide sufficient information to make important design decisions. 
Table 7-1. Program Comparison of the Four Computer Codes

\begin{tabular}{|c|c|c|c|c|}
\hline $\begin{array}{c}\text { Capabilities } \\
\end{array}$ & BLAST 3.0 & DOE 2.1 & DEROB 4 & SUNCAT 2.4 \\
\hline $\begin{array}{l}\text { Residential and commercial } \\
\text { applicability }\end{array}$ & Residential and commercial & Residential and commercial & $\begin{array}{l}\text { Residential, limited } \\
\text { commercial }\end{array}$ & Residential \\
\hline $\begin{array}{l}\text { Systems (e.g., furnaces, } \\
\text { chillers) }\end{array}$ & Yes & Yes & Limited & No \\
\hline Latent loads & Yes & Yes & No & No \\
\hline \multicolumn{5}{|l|}{ User interface } \\
\hline $\begin{array}{l}\text { User consultation and } \\
\text { support }\end{array}$ & $\begin{array}{l}\text { Available from time share } \\
\text { services }\end{array}$ & $\begin{array}{l}\text { Available from time share } \\
\text { services }\end{array}$ & $\begin{array}{l}\text { Available from code author on } \\
\text { contract basis }\end{array}$ & $\begin{array}{l}\text { Available from code author on } \\
\text { contract basis }\end{array}$ \\
\hline Documentation & Fair & Good & Fair & Poor \\
\hline Input & $\begin{array}{l}\text { Building design language, } \\
\text { extensive data libraries }\end{array}$ & $\begin{array}{l}\text { Building design language, } \\
\text { extensive data libraries }\end{array}$ & $\begin{array}{l}\text { Fixed format structure, } \\
\text { material properties libraries }\end{array}$ & Interactive input \\
\hline Output & $\begin{array}{l}\text { Monthly and yearly } \\
\text { summaries, hourly output, } \\
\text { design days, numerous other } \\
\text { output }\end{array}$ & $\begin{array}{l}\text { Monthly and yearly } \\
\text { summaries, hourly output, } \\
\text { design days, numerous other } \\
\text { output }\end{array}$ & $\begin{array}{l}\text { Monthly and yearly } \\
\text { summaries, hourly output, } \\
\text { detailed typical day output }\end{array}$ & $\begin{array}{l}\text { Monthly and yearly } \\
\text { summaries, hourly output }\end{array}$ \\
\hline Error messages & Input variable range checking & Input variable range checking & $\begin{array}{l}\text { Check for gross } \\
\text { inconsistencies }\end{array}$ & $\begin{array}{l}\text { Check for gross } \\
\text { inconsistencies }\end{array}$ \\
\hline $\begin{array}{l}\text { Run cost (one year, typical } \\
\text { passive structure on SERI } \\
\text { computer) }\end{array}$ & Inexpensive (\$8) & Inexpensive (\$5) & Expensive (\$20) & Inexpensive (\$5) \\
\hline Execution mode & Batch & Batch & Batch & Interactive \\
\hline $\begin{array}{l}\text { Time to create a building input } \\
\text { file }\end{array}$ & Longer & Longer & Longer & Shortest \\
\hline $\begin{array}{l}\text { Generality of building } \\
\text { geometry description }\end{array}$ & General & General & $\begin{array}{l}\text { Most general (limited to } 2120 \\
\text { temperature nodes, } 12 \\
\text { surfaces/volume }\end{array}$ & Limited (only one sunlit zone) \\
\hline \multicolumn{5}{|l|}{$\begin{array}{l}\text { Solution technique for heat } \\
\text { transfer equations }\end{array}$} \\
\hline Walls & Transfer functions & Response factors & $\begin{array}{l}\text { Implicit integration for wall } \\
\text { nodes }\end{array}$ & $\begin{array}{l}\text { Explicit integration for wall } \\
\text { nodes }\end{array}$ \\
\hline Zones & $\begin{array}{l}\text { Solution of set of linear or } \\
\text { nonlinear equations }\end{array}$ & Custom weighting factors & $\begin{array}{l}\text { Solution of set of linear or } \\
\text { nonlinear equations }\end{array}$ & $\begin{array}{l}\text { Solution of set of nonlinear } \\
\text { equations for two zones, } \\
\text { remaining zone temperatures } \\
\text { calculated explicitly }\end{array}$ \\
\hline \multicolumn{5}{|l|}{ Solar model } \\
\hline $\begin{array}{l}\text { Solar radiation and weather } \\
\text { input data }\end{array}$ & $\begin{array}{l}\text { Accepts TMY, TRY, } 1440 \text {, } \\
\text { and } 280 \text { format tapes. Can } \\
\text { use internal solar radiation } \\
\text { model. }\end{array}$ & $\begin{array}{l}\text { Accepts TMY, TRY, and } \\
\text { special California data tapes. } \\
\text { Can use internal solar } \\
\text { radiation model. }\end{array}$ & $\begin{array}{l}\text { Accepts direct and diffuse } \\
\text { solar radiation data. Expects } \\
\text { weather data in its own } \\
\text { format. Can use internal solar } \\
\text { radiation model. }\end{array}$ & $\begin{array}{l}\text { Accepts direct normal and } \\
\text { total horizontal solar radiation } \\
\text { data. Expects weather data in } \\
\text { its own format. No internal } \\
\text { solar radiation model }\end{array}$ \\
\hline Diffuse solar radiation model & Isotropic & Anisotropic & Anisotropic & Isotropic \\
\hline
\end{tabular}




\begin{tabular}{|c|c|c|c|c|}
\hline Capabilities & BLAST 3.0 & DOE 2.1 & DEROB 4 & $\begin{array}{l}\text { SUNCAT } 2.4 \\
\end{array}$ \\
\hline Ground reflectance & $\begin{array}{l}\text { Defined internal to the } \\
\text { program }\end{array}$ & $\begin{array}{l}\text { User-defined constant, one } \\
\text { for each external wall surface }\end{array}$ & $\begin{array}{l}\text { Defined internal to the } \\
\text { program }\end{array}$ & $\begin{array}{l}\text { User-defined constant (one } \\
\text { for each glazing type) }\end{array}$ \\
\hline \multicolumn{5}{|l|}{ Solar radiation } \\
\hline $\begin{array}{l}\text { External surface } \\
\text { absorptivity modeled }\end{array}$ & Yes & Yes & Yes & No \\
\hline $\begin{array}{l}\text { Distribution of radiation } \\
\text { onto internal surfaces }\end{array}$ & $\begin{array}{l}\text { Several levels of model } \\
\text { available, including one that } \\
\text { dynamically considers } \\
\text { geometry }\end{array}$ & $\begin{array}{l}\text { Area weighted or user-defined } \\
\text { constant split }\end{array}$ & $\begin{array}{l}\text { Approximate model that } \\
\text { dynamically considers } \\
\text { geometry }\end{array}$ & Area weighted \\
\hline Shadowing & $\begin{array}{l}\text { Shadowing for windows, } \\
\text { general shadowing if } \\
\text { requested }\end{array}$ & $\begin{array}{l}\text { Shadowing for windows, } \\
\text { general shadowing if } \\
\text { requested }\end{array}$ & $\begin{array}{l}\text { General shadowing for all } \\
\text { surfaces }\end{array}$ & Shadowing for windows \\
\hline Glass transmittance & $\begin{array}{l}\text { User defined or obtained from } \\
\text { library }\end{array}$ & Obtained from library & Defined internal to program & User defined \\
\hline Infrared radiation & $\begin{array}{l}\text { Approximate treatment using } \\
\text { "mean radiant temperature" } \\
\text { for internal surfaces only }\end{array}$ & $\begin{array}{l}\text { Considered when generating } \\
\text { custom weighting factors, } \\
\text { reradiation to sky } \\
\text { approximated }\end{array}$ & $\begin{array}{l}\text { General infrared radiation } \\
\text { network, sky temperature } \\
\text { considered for all external } \\
\text { surfaces }\end{array}$ & $\begin{array}{l}\text { Linearized infrared term } \\
\text { included as part of film } \\
\text { coefficient }\end{array}$ \\
\hline \multicolumn{5}{|l|}{ Convection } \\
\hline $\begin{array}{l}\text { Internal surface film } \\
\text { coefficient }\end{array}$ & $\begin{array}{l}\text { Function of wall orientation } \\
\text { and direction of temperature } \\
\text { difference }\end{array}$ & $\begin{array}{l}\text { User-specified constant for } \\
\text { each surface }\end{array}$ & $\begin{array}{l}\text { Function of surface and air } \\
\text { temperature }\end{array}$ & $\begin{array}{l}\text { User-specified constant for } \\
\text { each surface }\end{array}$ \\
\hline $\begin{array}{l}\text { External surface film } \\
\text { coefficient }\end{array}$ & $\begin{array}{l}\text { Function of wind speed and } \\
\text { user-specified surface } \\
\text { roughness }\end{array}$ & $\begin{array}{l}\text { Function of wind speed and } \\
\text { user-specified surface } \\
\text { roughness }\end{array}$ & $\begin{array}{l}\text { Function of wind speed and } \\
\text { surface and air temperature }\end{array}$ & $\begin{array}{l}\text { Constant coefficient input that } \\
\text { includes nonmassive } \\
\text { insulation }\end{array}$ \\
\hline \multicolumn{5}{|l|}{ Advection } \\
\hline Infiltration & $\begin{array}{l}\text { Constant or can include wind } \\
\text { speed and temperature } \\
\text { difference effects }\end{array}$ & $\begin{array}{l}\text { Constant or can include wind } \\
\text { speed and temperature } \\
\text { difference effects }\end{array}$ & Constant & Constant \\
\hline Natural interzone advection & Yes & No & Yes & Limited \\
\hline Fans & General & Limited & Limited & Limited \\
\hline \multicolumn{5}{|l|}{ Schedules } \\
\hline Internal gains & Yes & Yes & Limited & Limited to constant \\
\hline Night insulation & Yes & Yes & Limited & Limited \\
\hline Thermostats & Yes & Yes & Limited & Limited, no setback \\
\hline \multicolumn{5}{|l|}{ Specific systems } \\
\hline HVAC systems & Yes & Yes & No & No \\
\hline Finite capacity furnaces & Yes & Yes & Yes & No \\
\hline Trombe wall & Yes & No & Yes & Yes \\
\hline Rock bed & No & No & Yes & No \\
\hline Active solar systems & Yes & Yes & No & No \\
\hline Water wall & Yes & No & Yes & No \\
\hline
\end{tabular}




\begin{tabular}{|l|l|l|l|l|}
\hline \multicolumn{1}{|c|}{ Capabilities } & \multicolumn{1}{c|}{ DLAST 3.0 } & \multicolumn{1}{c|}{ DER 2.1 } & Yes \\
\hline Thermosiphon air panel & No & No & No \\
\hline Lighting & Yes & Yes & No \\
\hline Daylighting & Yes & No & No \\
\hline Phase-change materials & No & No & No \\
\hline Furniture thermal mass & No & Yes & No \\
\hline
\end{tabular}




\subsubsection{Solution Technique}

All four codes use a nodal network or transfer functions (or both) to solve the heat transfer equations governing the thermal performance of a building. They all assume that

- Material properties are isotropic and constant.

- Boundary conditions (air and ground temperatures and incident radiation) at a surface are isotropic.

- Heat flow through walls is one dimensional.

- Zone air is isothermal.

As a result, one temperature represents the temperature of an entire surface (except in SUNCAT 2.4 where surface temperatures are not computed), and one temperature represents the temperature of the air in a zone. DEROB 4 uses a nodal network and solves a complete set of simultaneous equations at each time step, a rigorous and costly method. BLAST 3.0 uses transfer functions for wall conduction and solves a simplified set of simultaneous equations to provide fairly rigorous modeling at a greatly reduced cost. DOE 2.1 uses transfer functions exclusively and an approximate, noniterative solution to the simultaneous heat transfer equations to provide the least costly simulation. However, some of the assumptions required for this solution strategy may not be acceptable to passive solar building designers.

SUNCAT 2.4 uses an explicit integration technique for internal wall nodes and solves for a maximum of two zone air temperatures simultaneously. The reduced scope addressed by SUNCAT 2.4 provides for inexpensive simulations; again, some of the assumptions required may not be applicable to passive solar designs.

\subsubsection{Radiation Modeling}

All four codes model solar radiation as a combination of direct beam and diffuse radiation. They obtain solar radiation data on an hourly basis from input weather files. BLAST 3.0 can use the solar radiation data on a Typical Meteorological Year (TMY) format tape (direct normal and total horizontal radiation). It can also merge data from a 280 format solar data tape with the data from a 1440 or Test Reference Year (TRY) format tape to produce an integrated weather file. (TRY and 1440 format tapes contain no radiation data themselves.) Finally, if no solar radiation data are available, BLAST 3.0 can create solar data using cloud cover data from surface data tapes and approximative functions.

DOE 2.1 can use TMY format data as well as the TRY format data or a special set of data for California climate zones collected by the California Energy Resources Conservation and Development Commission. The TRY and California data are combined with solar radiation data calculated using algorithms that consider cloud cover. DOE 2.1 does not use 280 format solar data tapes.

DEROB 4 requires the user to transform the weather data to its own format. It can accept direct normal and diffuse horizontal radiation data. (Diffuse horizontal radiation can be calculated from TMY data.) If no solar radiation data are available, DEROB 4 can calculate the data using a model that includes air temperature, pressure, and relative humidity as well as an approximation of Liu-Jordan data [2]. 
SUNCAT 2.4 has no internal model to provide solar radiation data. It expects direct normal and total horizontal radiation data in its own format to be provided on an input weather file. BLAST 3.0 and SUNCAT 2.4 use isotropic diffuse sky radiation models; DEROB 4 and DOE 2.1 use anisotropic models. All are capable of modeling window shading devices; all but SUNCAT 2.4 can model the shading of one exterior surface by another. Ground reflectance is a user-specified constant for each wall or window for DOE 2.1 and SUNCAT 2.4. It is set internally by BLAST 3.0 and DEROB 4. The physical properties of the window glazings can be user-specified in all the codes except DEROB 4. In DEROB 4, the user must select from a library of window types. Once the radiation has entered a zone, SUNCAT 2.4 and DOE 2.1 allocate a constant fraction to the various internal surfaces; BLAST 3.0 and DEROB 4 can trace the beam radiation to the surfaces it would strike. BLAST 3.0 is the only code that allows the user to specify the fraction of incoming solar radiation that can be used to reduce the energy demand caused by lighting requirements (daylighting).

DEROB 4 explicitly models the nonlinear infrared radiation exchange between interior surfaces and between the exterior surfaces and the ground and air. BLAST 3.0 does not model the infrared radiation exchange for exterior surfaces but approximates the infrared radiation exchange for internal surfaces with a mean radiant temperature for each surface. Each internal surface is assumed to radiate to a fictitious surface that has about the same heat transfer from the internal surface as in the real multi-surface case. DOE 2.1 includes linearized infrared radiation exchange in generating the weighting factors that characterize a room. It also uses a simple model to approximate the infrared radiation exchange between an exterior surface and the sky. SUNCAT 2.4 contains no explicit infrared radiation modeling.

\subsubsection{Convection and Conduction Modeling}

The film coefficients in DEROB 4 are functions of surface orientation, wall and air temperatures, and the speed of air passing the surface. In BLAST 3.0 the interior film coefficients are functions of surface orientation and the direction of heat flow at the surface (into or out of the wall); exterior film coefficients are functions of wind speed and surface roughness. Exterior film coefficients in DOE 2.1 are also functions of wind speed and surface roughness, while interior film coefficients are user specified constants. Note that film coefficients for DOE 2.1 are expected to contain an approximation of the infrared heat transfer at the wall surface. The program automatically separates infrared and convective contributions when generating custom weighting factors. Film coefficients can be included only as constants in SUNCAT 2.4.

The infiltration rate in BLAST 3.0 and DOE 2.1 can be a constant or a function of inside/outside air temperature differences and wind speed. It can also be scheduled on an hourly basis. The infiltration rate in DEROB 4 and SUNCAT 2.4 is input as a constant.

Limited models for natural advection between zones in BLAST 3.0, DEROB 4, and SUNCAT 2.4 allow for the modeling of Trombe walls. Interzone advection can be treated only as a constant in DOE 2.1.

Conduction is treated by transfer functions in BLAST 3.0 and DOE 2.1, while internal wall nodes are used in DEROB 4 and SUNCAT 2.4. The wall node temperatures are integrated explicitly in SUNCAT 2.4 and implicitly in DEROB 4. 
Ground coupling in the four codes is treated similarly to coupling to ambient air. However, the ground temperature is not read from the input weather file. Ground temperatures are input as a sine wave in SUNCAT 2.4 (one year period). They are input on a monthly basis in BLAST 3.0 and DOE 2.1. DEROB 4 calculates ground temperature each day as the average ambient air temperature for that day. The walls connected to the ground are specified as any other wall. DEROB 4 adds a resistance between the wall's exterior surface and the ground approximately equal to the thermal resistance of 10 feet of dry soil. BLAST 3.0, DOE 2.1, and SUNCAT 2.4 add no resistance and the user must input all resistances between the wall and the ground as part of the wall specification. The effects of the ground's thermal capacitance can be treated only by creating an earth "wall" to represent the ground in closest contact with the building.

\subsubsection{Systems and Schedules}

BLAST 3.0 and DOE 2.1 can simulate the performance of heating and cooling equipment including active solar systems and can calculate thermal loads. They can also consider the effects of latent loads due to humidity in the air on building energy use. DEROB 4 can simulate the effects of finite capacity heating and cooling equipment but not the performance of the equipment. SUNCAT 2.4 provides only the thermal loads (the heating and cooling capacity is considered infinite). DEROB 4 and SUNCAT 2.4 do not consider latent loads.

BLAST 3.0 and DOE 2.1 have extensive capability to schedule internal gains. DEROB 4 allows one set of 24 hourly internal gains to be used for the whole simulation. In SUNCAT 2.4 the internal gains are constants.

The effects of lighting on energy requirements can be simulated by BLAST 3.0 and DOE 2.1. BLAST 3.0 can also simulate the effects of daylighting on lighting requirements. Trombe walls can be simulated explicitly by BLAST 3.0, DEROB 4, and SUNCAT 2.4. Water walls are explicitly modeled by BLAST 3.0 and DEROB 4. Rock beds are explicitly modeled by DEROB 4. None of the codes explicitly models phase change materials or thermosiphon air panels.

Night insulation can be simulated by all four codes. Night setback can be simulated by all but SUNCAT 2.4. BLAST 3.0 and DOE 2.1 can vary the transmittance of shading surfaces as a function of the time of year; shading transmittances are constant in DEROB 4 and SUNCAT 2.4.

All the codes except SUNCAT 2.4 can simulate economizer cycles. BLAST 3.0 and SUNCAT 2.4 can simulate forced convection between zones based on a prescribed temperature difference between the zones. BLAST 3.0 can also simulate an hourly schedule for forced convection between zones.

\subsubsection{Passive Solar Applications}

Several building energy analysis capabilities are particularly important in dealing with passive strategies for energy conservation. First, it is important to find the correct amount of sunlight entering an interior zone. All the codes consider the direct beam portion of the sunlight in detail. However, the diffuse component of the solar radiation is also important to the performance of passive solar designs. DEROB 4 and DOE 2.1 use anisotropic sky models to produce the diffuse radiation, concentrating more diffuse radiation near the sun's position. SUNCAT 2.4 and BLAST 3.0 use isotropic sky models. Anisotropic sky models can easily be added to either 
BLAST 3.0 or SUNCAT 2.4. Second, the ground reflectance is a constant in all four codes, so the additional solar radiation gain caused by snow in the winter is neglected. Variable ground reflectances could be easily added to all the codes. One innovative passive strategy is the use of reflecting surfaces to increase the amount of solar radiation collected by the windows. None of the codes currently has the capability to model this strategy, but it could be added to all the codes for specific reflector geometries. All the codes calculate window shading sufficiently.

Once the radiation has entered a zone in a passive solar building, it is important that the radiation strike a mass wall - a wall with sufficient thermal mass (such as brick, tile, concrete, or water) to act as an energy storage device. DEROB 4 and BLAST 3.0 can trace the direct beam solar radiation to the interior surface it would strike. DOE 2.1 and SUNCAT 2.4 do not. DOE 2.1 allows the user to select the portion of radiation striking each surface on an average basis, but SUNCAT 2.4 assumes that all radiation strikes specified mass walls and distributes it on an areaweighted basis between those walls. Adding beam tracing to DOE 2.1 would be reasonably straightforward since it can already model the general shading of one exterior surface by another. However, it would be difficult to add to SUNCAT 2.4 since the current shading model in SUNCAT 2.4 is more limited.

Another use of the solar radiation entering a zone is for daylighting. Currently, BLAST 3.0 allows the user to specify the portion of the solar radiation entering a zone that may be used to offset lighting energy requirements. The other codes could be easily modified to add this capability. More detailed analysis of daylighting (calculation of the total illumination in the room) could be added easily only to DEROB 4 since DEROB 4 rigorously calculates view factors and treats multiple reflections.

Another common passive solar technique is the use of innovative window glazing to decrease the heat loss through the windows in the heating season or to decrease the solar heat gain through the windows in the cooling season. All the codes allow the user to include the physical properties of the windows except DEROB 4. DEROB 4 allows only glazings specified from its internal library; however, it would not be difficult to add more glazing types to the program. One particular innovative glazing is a selective surface that transmits visible radiation but reflects infrared radiation and decreases the heat loss through a window. Another example of a selective surface is a coating used as part of a Trombe wall. A Trombe wall surface should absorb a maximum amount of visible radiation while emitting a minimum amount of infrared radiation. DOE 2.1, BLAST 3.0, and DEROB 4 model infrared radiation, but only BLAST 3.0 allows the user to specify infrared absorptivity for the surfaces. A minor change in the other two codes would allow a user to input these quantities. SUNCAT 2.4 does not treat infrared radiation, and modifying it to explicitly model selective surfaces would require a major change. Note that infrared radiation exchange between interior wall surfaces is very important in passive solar designs because the temperatures of some wall surfaces (mass walls and windows) differ substantially with the other surfaces.

Since the walls and floor of a passive solar design are commonly used as energy storage devices, a building energy analysis code should model their thermal capacitance. All the codes model wall thermal capacitance. One innovative passive design technique, currently not modeled by the codes, is the use of phase-change materials in walls to store energy. A phase-change material 
stores energy by changing from one phase to another (e.g., solid to liquid) when energy is added. The effect is nonlinear. Some additions of energy cause temperature changes, some cause phase changes. DOE 2.1 is the only code for which linearity is a pervasive assumption. Modifying DOE 2.1 to model phase-change materials would be very difficult. BLAST 3.0 uses transfer functions to model walls (linearity assumed) but also uses surface and air temperature nodes that are determined by solving simultaneous, nonlinear, algebraic equations. Adding phase-change materials to BLAST 3.0 would be cumbersome but not impossible. DEROB 4 and SUNCAT 2.4 use temperature nodes to model walls; similarly, it would be cumbersome but not impossible to modify them to model phase-change materials.

Another passive design option is the water wall, which is a wall made up of containers filled with water. This device can be adequately modeled by all the codes by defining a wall with the appropriate high thermal conductance and high thermal mass.

The wall models in the codes can also be used to model the effects of thermal capacitance in the ground under the building. The ground temperatures specified in the codes are used much like the ambient air temperature. A wall or floor is in contact with the ground at that temperature, but the wall has no effect on the ground temperature. To simulate the mechanism by which the ground near a building heats up and stores energy, a ground "wall" must be defined. Correctly defining and using such walls requires substantial engineering judgment. The difficulty of treating local terrain effects has resulted in ground thermal capacitance not being included specifically in any of the codes.

One major passive design strategy involves collecting and/or storing heat in one part of a building and then using fans to move it to another part of the building when necessary and when possible. The determination of whether a transfer of heat is necessary and possible is usually based on temperature differences. One example is an attached sunspace with a rockbed. The sunspace is an added room designed to collect a large amount of solar radiation. If the sunspace gets too warm, a fan blows air from the sunspace over a bed of rocks to cool the sunspace and heat the rocks. If other rooms need heat, air is blown either directly from the sunspace to the rooms or through the rock bed to the rooms needing heat.

The use of fans with Trombe walls and thermosiphon air panels is similar to the use of fans with a sunspace. In a Trombe wall, a small space is bounded by glazing on one side and a mass wall on the other. In a thermosiphon air panel, a small space is bounded by glazing on one side and an ordinary wall on the other (usually an inexpensive retrofit option). Both systems can be designed to use natural advection to move heat from the small space to an adjoining room. They can also be designed to use fans. To model this set of options requires that a code contain the logic to switch the fans on and off and the ability to solve for the temperatures of several zones simultaneously. BLAST 3.0, DEROB 4, and SUNCAT 2.4 can solve for zone temperatures simultaneously. Each of these three codes contains some logic to implement the above passive strategies. Altering the logic or adding to it is not difficult. However, if the zone temperatures are not solved simultaneously at each time step, the strategies cannot be accurately simulated. DOE 2.1 does not solve for zone temperatures simultaneously, and changing DOE 2.1 to allow for these strategies would require substantial changes to the program or substantial approximation. 
Two strategies that can be considered passive or conservation strategies are the use of night insulation and thermostat setback. Insulation is placed over windows to reduce heat loss when the sun is not shining. Night setback refers to the practice of lowering thermostat settings at night and raising them in the morning. In general, all the codes have some capability to simulate these strategies. However, SUNCAT 2.4 cannot simulate night setback. DEROB 4 has limited capabilities for simulating both strategies. BLAST 3.0 and DOE 2.1 contain the most general capabilities. Adding the capability to simulate either option is not difficult.

Finally, although heating and cooling equipment is predominantly backup for passive solar applications, it should be mentioned here. Ideally, the performance of heating and cooling equipment should be simulated simultaneously with the building thermal response. However, BLAST 3.0 and DOE 2.1 simulate the equipment performance based on the results of the thermal response calculations. Changing this strategy would require significant structural changes in the program. DEROB 4 contains only limited equipment simulation, and SUNCAT 2.4 contains no equipment simulation. The structure of both codes allows for simultaneous equipment/thermal response simulation, but adding the large number of equipment options contained in BLAST 3.0 and DOE 2.1 would require a major effort.

\subsection{SUNCAT 2.4}

SUNCAT 2.4 is a program generally suited to analyzing the thermal response of small, simple buildings. It is able to simulate the hourly temperature variation in a building and to determine the amount of energy required to keep the interior of the building between an upper and a lower set point. However, it has no capability for simulating specific heating and cooling devices (e.g., furnaces and chillers), considering latent loads, or performing economic analyses for various designs. These capabilities are generally desired when analyzing commercial buildings.

\subsubsection{User Interface}

SUNCAT 2.4 requires a fairly knowledgeable user. The inputs to the program are in engineering units; for example, wall thermal properties rather than the material making up a wall. Additionally, the documentation for the program is sparse and contains no examples of input files. The program is applicable to a limited range of problems. Only one zone may be sunlit or contain massive walls, and there may be no more than five zones in all. As a result of the limited scope, SUNCAT is easy to learn. The interactive input sequence leads a novice user through the necessary input steps. However, the limited scope also requires that substantial engineering judgment be exercised to model even the most ordinary residential structure.

SUNCAT 2.4 runs interactively on the computer. The user modifies inputs to and controls the execution of the program while it is running. This characteristic makes the program relatively easy to use but requires the user to remain at the computer terminal while the program is running. Additionally, some computer systems charge significantly more for interactive execution than they do for batch execution. In batch execution, the input files are prepared in advance and submitted with the program as a job, which may be run at any time. Simulating a single zone direct-gain building for one year (on the SERI computer system) using SUNCAT 2.4 costs about $\$ 5.00$. 
The program has two types of output: monthly and yearly summaries for various energy gains and losses, and hourly radiation and temperature variations. The only flexibility that exists in choosing output data is the ability to eliminate the hourly output data.

\subsubsection{Solution Technique}

SUNCAT 2.4 solves the heat transfer equations governing the thermal performance of a building by setting up a nodal network. That is, the temperature variation throughout the building is approximated by the temperature at a limited number of points (nodes) within the building. In particular, SUNCAT 2.4 uses one node for each zone temperature and the user must specify the number of nodes to be used to characterize each wall. Usually, the more massive the wall, the greater the number of nodes needed to accurately characterize it.

The program uses an explicit integration technique to calculate the temperatures of the wall nodes. That is, the temperature for a wall node at any given time is calculated using its temperature and the temperatures of surrounding nodes from the previous point in time. Once the wall temperatures have been computed at the current time, the zone temperatures are calculated using heat balance equations. The amount of heat entering the zone balances the amount leaving the zone. In the absence of advective coupling, this solution strategy allows SUNCAT 2.4 to proceed without having to iterate to solve the equations at each time step. The program iterates to solve simultaneously for the temperature of the sunlit zone and one other zone when advective coupling is indicated. The advantage of the SUNCAT 2.4 approach is that the program executes quickly and thus inexpensively. The disadvantage is that the simultaneous interactions between the walls and the zones have been simplified. This lessens the accuracy of the solution and in particular of modeling convection.

The choice of explicit integration for the wall nodes limits the time step that may be chosen. Once the user chooses the number of nodes for each wall, the second law of thermodynamics dictates an allowable maximum time step. The user must choose the time step, but SUNCAT 2.4 informs him or her if the choice is unacceptable. More wall nodes imply a shorter time step, which implies longer and more expensive computer runs.

\subsubsection{Radiation Modeling}

SUNCAT 2.4 expects solar radiation data (direct normal and total horizontal radiation) to be provided as part of the input weather data. The solar radiation striking the building is modeled as a combination of direct beam and diffuse radiation. The diffuse contribution is isotropic, that is, the diffuse radiation intensity is the same for each portion of the sky. The radiation striking the building affects only the windows in the sunlit zone; the heat absorbed by the exterior walls is not modeled. The windows also receive radiation reflected from the ground; however, ground reflectance is a user-specified constant for each window and cannot reflect seasonal variations (e.g., the effect of snow).

A maximum of 10 windows may be modeled for the sunlit zone. Each window may have multiple layers of the same glazing material, and the user specifies the optical properties of a single layer. These properties, along with the angle at which the radiation strikes the window, are used to calculate the amount of radiation transmitted through the window. For diffuse radiation, the user inputs an equivalent incidence angle. 
A window may face any direction and a variety of shading devices may be specified for it such as overhangs and mullions. The shading devices are considered infinite in length so that edge effects are not considered. Shading of one part of the building by another part is not modeled. Once the radiation entering the sunlit zone has been calculated, a constant fraction (user input) is lost, representing the radiation reflected back out the windows (cavity albedo effect). Another constant fraction (user input) is transferred directly to the air, representing the energy absorbed by furnishings and quickly transferred to the air through convection. The remainder is allocated on an area weighted basis to choose walls that contain mass.

No explicit infrared radiation modeling is done in SUNCAT 2.4. However, the user may approximate infrared radiation losses (or gains) by including an extra contribution when specifying surface film coefficients.

\subsubsection{Convection and Conduction Modeling}

SUNCAT 2.4 does not allow the user to model walls made of multiple massive layers, as DOE 2.1, BLAST 3.0, and DEROB 4 do. Each wall is assumed to contain one homogeneous mass material. Conduction is modeled in two different ways. For mass walls with one side facing the sunlit zone, conduction is treated as part of the nodal equations discussed earlier. All other walls are treated as pure thermal resistances between one zone and another or between one zone and the ground or ambient air. The pure resistance accounts for all conductive and convective coupling due to the wall. Convective couplings between the walls and the zones they enclose are always user-specified constants. When one of the walls is bounded by the ground, the coupling coefficient should represent not only the resistance due to contact between the wall surface and the ground, but also any resistance meant to represent an effective ground thickness that intervenes before the ground temperature is "seen" by the wall surface. The thermal capacitance of the ground is not explicitly treated. Ground temperature is input as a sinusoidal function with a period of one year.

Coupling coefficients can also be used to describe a constant advective coupling between zones. However, specifying constant values for convective and advective couplings precludes the ability to model time varying effects such as the sensitivity of film coefficients to temperature and wind speed. SUNCAT 2.4 has the ability to model a free convective loop between the sunlit zone and one other zone, specifically intended for modeling a Trombe wall with vents. The coupling for this loop is temperature dependent.

\subsubsection{Systems and Schedules}

SUNCAT 2.4 can model a window shutter schedule that opens and closes the shutters (that have a thermal resistance) according to several criteria. Also, forced convection between the sunlit zone and one other zone may be controlled automatically so that heat is moved from the sunlit zone to the other zone under appropriate conditions. These are the only items within the program that can be scheduled. Internal gains (e.g., appliances) and thermostat set points are constants. Night setback cannot be simulated.

The only specific system modeled in SUNCAT 2.4 is the Trombe wall. Rock beds, water walls, phase change materials, and thermosiphon air panels are not. 
In summary, SUNCAT 2.4 is designed primarily to compute the thermal loads for passive residential buildings and has only limited capability for modeling devices and systems.

\subsection{DEROB 4}

DEROB 4 is a program that models in detail the various heat transfer mechanisms of a building. It allows the user to input a building description without knowing the physical properties of the building materials. The program is designed to model buildings of arbitrary shapes and sizes with few restrictions on building geometry. The number of walls and the number of zones allowed are limited. A substantial amount of input is necessary to adequately describe the building for this code. DEROB 4 also uses a rigorous solution technique that causes relatively long execution times. As a practical matter, therefore, this code is primarily applicable to residential and small commercial buildings. It can model finite capacity heating and cooling devices, and can schedule some night insulation, internal gains, and thermostat settings. It does not consider latent loads. Hourly temperature variations and monthly and yearly heating and cooling loads can be obtained. DEROB 4 has no capability to perform economic analyses.

\subsubsection{User Interface}

Theoretically, DEROB 4 can be run by users without an engineering background. The inputs describe the geometry of the building and specify which materials are used in its construction. However, the amount of input becomes tedious and time consuming when a detailed representation of a building is input. As a result, a user is likely to make assumptions to simplify the building description input to the program.

The code requires four formatted input files (each item has to be placed in its proper column). Thus, preparing the input for DEROB 4 is a more tedious and error prone process than it is for programs with free format input (SUNCAT 2.4) or input languages (BLAST 3.0, DOE 2.1). DEROB 4 consists of six programs that run in the batch mode. The user prepares the input files and then submits a job for processing. It is relatively expensive to run, costing about $\$ 20$ to simulate a single zone direct-gain building for one year (on the SERI computer system); however, the program has several options that allow the user to decrease the modeling level of detail and thus speed execution.

DEROB 4 provides a large amount of output describing the properties of the building. For example, the output contains the fraction of each wall surface visible to the other wall surfaces, and each wall's conductance and time constant. Additionally, the hourly solar radiation entering each zone and striking each wall is available for a representative day each month. Hourly values for zone temperatures and the energy used to heat or cool each zone are available. A detailed description of the hourly heat flow rates on all interior surfaces can be obtained for the middle day each month. Monthly and yearly totals for the energy used to heat and cool the building are also provided.

\subsubsection{Solution Technique}

DEROB 4 solves the heat transfer equations governing the thermal performance of a building by setting up a nodal network. One node represents the temperature of each zone (a zone may be filled with air, water, or rock) and there is one node for each surface of the building. Finally, the program automatically selects the number of nodes that represent the temperature variation in the 
interior of the walls. Thus, the program may use no nodes for the interior of a wall that has little thermal mass.

The program uses an implicit integration technique to calculate nodal temperatures; that is, the temperature of the node for any given time is calculated using temperatures from the previous point in time and current temperatures. The wall surface nodes and the air zone temperatures are calculated using heat balance equations, so that heat entering the node is balanced by the heat leaving the node. As a result of this strategy, DEROB 4 must iteratively solve the equations for the internal, surface, and zone nodes at each time step. However, the program may always use a one hour time step without violating the second law of thermodynamics. The advantage of the DEROB 4 strategy is that temperature-dependent variations in the convective and advective couplings may be modeled explicitly. Film coefficients, infrared radiation, and free advection between zones are examples of temperature-dependent couplings.

\subsubsection{Radiation Modeling}

DEROB 4 can accept direct and diffuse radiation data from an input weather file or calculate them internally. It calculates direct normal radiation using an internal model that considers temperature, pressure, and relative humidity, and diffuse horizontal radiation using an approximation of data reported by Liu-Jordan [2].

The diffuse portion of solar radiation is anisotropic, concentrating radiation near the sun's position. Also, some radiation is reflected diffusely by the ground. The ground reflectance is set within the program and is the same value for all walls and windows (0.1). Seasonal variations in ground reflectance and different reflectances for different sides of the building cannot be modeled. DEROB 4 models the shading of any surface within the building by another surface. The shaded portion of a surface is approximated by dividing it into a 3 by 3 set of smaller surfaces. If the center of a smaller surface is shaded, the entire smaller surface is considered to be shaded. Shading patterns are calculated for each hour once per month and used for the whole month.

Since surfaces of arbitrary shape can be constructed, the program is set up to model general shading schemes. Solar radiation is absorbed and reflected by every surface that it strikes, including windows and exterior and interior walls. After the first reflection, all radiation is considered diffuse, with full treatment given to multiple reflections within a zone. The absorptivity of each opaque surface is a user input.

The type of windows in the building is specified by choosing either single, double, or triple glazing. The optical properties of the window are then taken from an internal program library. Thus specification of the type of windows is easier, but glazing schemes that may be simulated are limited. Additionally, diffuse radiation is treated as normal to the window in DEROB 4, whereas in most other codes some other effective incidence angle is chosen. This treatment will lead to larger values for transmitted diffuse radiation for a given set of glazing properties.

DEROB 4 fully models the nonlinear infrared radiation exchange between pairs of wall surfaces and between exterior surfaces and the sky and exterior surfaces and the ground. Multiple reflections are considered for interior surfaces. For exterior surfaces, a sky temperature is 
calculated using an algorithm from Gates [5], and the reflection of infrared radiation by the ground is considered. This capability can be important in passive solar buildings, in which walls may differ significantly in temperature and in passive cooling designs.

\subsubsection{Convection and Conduction Modeling}

The film coefficients in DEROB 4 are a function of the temperature of the wall surface, the orientation of the wall (e.g., ceiling, floor, vertical wall), the temperature of the air with which the surface is in contact, and the speed of the air passing the surface. The air speed for exterior surfaces is the wind velocity from the input weather file. Interior air speed is set to a constant 2 mph. This level of detail more closely resembles the real world than setting the coefficients to constants. In particular, the film coefficients have an important effect on the heat loss through the windows, and the temperature differences between the window surface and the interior air are considerable.

DEROB 4 also contains a model of the coupling due to natural advection. The model is dependent on the height difference between two openings in a wall or the vertical length of a single opening. It is also dependent on the temperature difference between the coupled zones. The DEROB strategy, which solves for all node temperatures simultaneously at each time point, allows convective and advective modeling at this level of detail. Note also that a building with several coupled zones will require a large amount of computer time to simulate.

Conduction within the walls is handled by implicit integration of the temperature of the wall nodes. DEROB 4 automatically selects the number and the spacing of nodes within the wall according to its own internal check of the resulting solution accuracy. If the wall has little mass (e.g., interior gypsum board walls in a residential structure), DEROB 4 may choose to use no nodes, making the wall a pure resistance. Ground coupling in DEROB 4 is handled similar to coupling to ambient air. A wall connected to the ground is specified just as any other wall. However, instead of an external film coefficient, an external resistance approximately equal to the thermal resistance of 10 feet of dry soil 1s added to the wall resistance. Ground temperature is calculated each day as the average ambient air temperature for that day. The thermal capacitance of the ground is not explicitly considered.

\subsubsection{Systems and Schedules}

DEROB 4 can model both finite and infinite capacity heating and cooling equipment. It can model economizer cycles and actively charged rock beds (although the documentation for these two capabilities must be obtained from the source code for the program). It can also model Trombe walls and water walls.

DEROB 4 allows the user to specify different values for internal gains for each hour of the day; the same set of 24 values is used for each day of the year. Night setback may be scheduled for both cooling and heating set points. Physical properties of night insulation may be specified separately for each window. Night insulation can then be scheduled (on a monthly basis) to be in place during the night or during the day. As a result of these capabilities, major passive heating and cooling schemes can be modeled. Also, the generality of the DEROB 4 solution technique would allow new passive algorithms to be included easily. 
DEROB 4 lacks the capability to schedule fans other than those used for the rock beds and economizer cycles. Other fans between zones are assumed on all the time. Thus, a fan used to transfer heat from a sunspace to the remainder of a building on an intermittent basis cannot be modeled.

\subsection{BLAST 3.0}

BLAST 3.0 is a flexible program designed to analyze the thermal response and energy consumption of residential and commercial buildings. In particular, the program models thermal processes in sufficient detail that passive solar designs may be evaluated while retaining computational efficiency. In addition to computing temperatures and thermal loads, it can simulate the equipment used to produce and distribute heating and cooling to the spaces being conditioned (including active solar systems). It also considers latent loads. The program can compute life-cycle costs including capital, operation, and energy costs, thus allowing the user to assess the cost-effectiveness of energy conserving options.

\subsubsection{User Interface}

Users describe their building to BLAST 3.0 with a building description language. The input format is easy to use and allows written comments within the input file. The input language allows great flexibility and power in preparing a building description. The generality and flexibility of the input can be intimidating to a novice user; however, the documentation provides a sample problem and should ease the burden of learning the input procedure. The program executes in the batch mode. The user prepares the input file and then submits the job for processing. The input building description is automatically checked for errors. If critical input errors are found, the program is aborted. Simulating a single zone direct-gain building for one year on the SERI computer system using BLAST 3.0 costs about $\$ 8.00$.

The output from the program is fairly extensive and includes detailed wall properties. Yearly and monthly energy use summaries are printed for each zone and for the whole building. Peak loads are also printed. Additionally, hourly output for many variables is available, excluding hourly loads associated with individual walls and windows. Various other reports are available; for example, a summary of the monthly and yearly loads not met by the heating/cooling equipment, and printer plots showing a plan view of each zone. Reports associated with the economic analyses are available but they are not discussed here.

\subsubsection{Solution Technique}

BLAST 3.0 uses a nodal network and transfer functions [6] to solve the heat transfer equations governing the thermal performance of a building. The transfer functions relate the current values of wall surface temperatures and heat flow rates through the walls to past values. The nodal network relates the temperature and heat flow rates at each surface to each other and to the zone air temperatures. Additionally, the radiant interchange between the surfaces of a zone is modeled by assuming that each surface radiates to a fictitious surface that has about the same heat transfer from the surface as in the real multi-surface case [6]. The program uses successive substitution to solve for all the surface and air temperatures simultaneously. As a result, BLAST 3.0 can account for nonlinear, nonconstant convection coefficients and heat transfer between simultaneously simulated rooms, while retaining computational efficiency. Thus, it provides an accurate and economical simulation of passive heating and cooling schemes. 


\subsubsection{Radiation Modeling}

BLAST 3.0 can accept solar radiation data from a TMY or 280 format data tape. It can also calculate solar radiation data using cloud cover data from a TRY or 1440 format weather tape along with approximative functions.

The solar radiation model in BLAST 3.0 calculates the radiation striking the building as a combination of direct beam and diffuse radiation. The diffuse sky radiation model is isotropic. That is, the diffuse radiation intensity is the same for each portion of the sky. Also, some radiation is reflected diffusely by the ground. Ground reflectance is set within the program so that seasonal variations in ground reflectance (snow) are not modeled. BLAST 3.0 models the shading of a window by wings and overhangs; it also allows the shading of one surface by another surface. The flexibility exists to model only important shading surfaces, neglecting the effects of minor shading. Additionally, the transmittance of a surface may be changed with the season, thus allowing the simulation of variable shading mechanisms such as trees. Hourly shading patterns are calculated for at least 14 periods throughout a year (more if shading changes do not coincide with these periods). A constant shading pattern is used throughout each period. The shading calculations are exact for surfaces that can be represented as convex polygons. BLAST represents its surfaces as sets of convex polygons.

To determine the amount of radiation that enters the building through the windows, the program uses a solar transmission algorithm developed from the fundamental physics of the process. The physical characteristics of the window may be input by the user, or the window may be selected from a library. Once the solar radiation has entered a window, the program provides two options for the distribution of radiation onto interior surfaces. The simple option assumes that all the solar radiation is incident on the floor. The solar radiation not absorbed by the floor is added to the diffuse radiation, which is uniformly incident on all interior surfaces. The detailed option calculates the beam radiation falling on each interior surface. After the first reflection, beam radiation is combined with diffuse radiation and distributed to the interior surfaces on an areaemissivity-weighted basis. A portion of this diffuse radiation distributed to the inside of the windows is transmitted out of the building (cavity albedo effect). Multiple reflections are not modeled.

Infrared radiation exchange between internal surfaces is modeled by assuming that each surface radiates to a fictitious surface that has about the same heat transfer from the surface as in the real multi-surface case [6]. The fictitious surface is considered to enclose the original surface completely, and multiple reflections between the two are considered. Infrared radiation exchange is not explicitly modeled for exterior surfaces but can be approximated by adding an extra contribution to the exterior film coefficients.

\subsubsection{Convection and Conduction Modeling}

The film coefficients for interior surfaces in BLAST 3.0 are functions of the orientation of the surface (e.g., ceiling. floor. wall) and the direction of heat flow (i.e., whether the surface or the air is warmer). The exterior film coefficients are functions of the wind speed and the userspecified roughness of the surface. Without changing the structure of the program, the film coefficients could become functions of the magnitude of the surface/air temperature difference. 
Infiltration may be input as a constant value. a function of the difference between inside and outside air temperature and/or a function of the wind speed. Additionally, the infiltration that has been calculated by one of these options may be scaled on an hourly basis by a user input schedule.

The program also can model the mixing of air from one zone with the air in another based on the temperature difference between the zones and on a user-specified schedule. This strategy is often used in passive designs.

Conduction in BLAST 3.0 is handled through transfer functions. Transfer functions relate current values of temperature and heat flow rate at the wall surfaces with past values. They simulate the effects of thermal mass in the wall. A user may create walls for a simulation or select walls from the program library. Nonmassive walls may appear as pure resistances.

To compute the heat exchange with the ground, ground temperatures are needed. The average ground temperature for each month is input by the user. Any thermal resistance from the ground (i.e., between an exterior wall surface and the ground temperature) must be input as part of the wall specification. The thermal capacitance of the ground is not explicitly considered.

\subsubsection{Systems and Schedules}

In addition to calculating the thermal loads and temperatures for the building, BLAST 3.0 has the ability to simulate the performance of the heating and cooling equipment in meeting these loads. Thus, the program not only simulates the effects of finite sized equipment, but also the efficiency with which the equipment operates. Among the types of equipment that can be simulated are active solar heating devices for space or water heating. BLAST 3.0 can also simulate the effects of artificial lighting on building energy requirements and the effectiveness of daylighting in reducing those requirements. Daylighting is modeled by allowing the user to specify (on an hourly basis) the fraction of the incoming solar radiation used to replace artificial lighting. The program contains explicit models for Trombe walls and water walls; it does not explicitly model rock beds, phase change materials, or thermosiphon air panels.

The program has extensive capability to simulate schedules. Internal gains (such as people, equipment, and lighting) can be individually scheduled. Night insulation and shading can be scheduled for each window. Economizer cycles can also be modeled.

As a result of the program's structure and internal models, BLAST 3.0 can simulate most passive solar designs. New passive simulation algorithms can be added without significantly changing the main program.

\subsection{DOE 2.1}

DOE 2.1 is a flexible program designed to analyze the thermal response and energy consumption of residential and commercial buildings. In addition to computing temperatures and thermal loads, it can simulate the equipment used to produce and distribute heating and cooling to the spaces being conditioned. It can also consider latent loads. The program can compute lifecycle costs including capital, operation, and energy costs, thus allowing the user to assess the cost effectiveness of energy-conserving options. 
However, because it makes a number of assumptions to allow the program to execute quickly, DOE 2.1 may not be suitable for the simulation of multi-zone passive heating and cooling schemes.

\subsubsection{User Interface}

Users describe their building to DOE 2.1 through the use of a building description language. The input format is easy to use and allows written comments within the input file. The input language allows great flexibility and power in preparing a building description.

The generality and flexibility of the input can be intimidating to a novice user; however, the documentation provides a sample problem and should make input preparation straightforward. DOE 2.1 can accept simple or detailed building descriptions. It does not require the description to be complete, but will analyze whatever part of the building is described. This capability is useful for preliminary design studies but leaves the burden of selecting the appropriate level of detail with the user.

The program executes in the batch mode. The user prepares the input file and then submits the job for processing. DOE 2.1 consists of two programs: one to process the input building description and one to perform the calculations. If there are errors in the building description, the second program is not executed. Simulating a single zone direct-gain building for one year on the SERI computer system using DOE 2.1 costs about $\$ 5$.

The output from the program is extensive and flexible. Yearly and monthly energy use summaries may be obtained for the whole building and the individual zones within the building. Peak loads are available, as are hourly gains and losses for individual walls, windows, and zones. Indeed, most variables may be output on an hourly basis.

Various other reports are available; for example, the hourly distribution of demand for heat from the heating equipment, and the number of hours during which the heating equipment could not meet demand. Reports associated with the economic analyses are available but they are not discussed here.

\subsubsection{Solution Technique}

DOE 2.1 solves for the hourly temperatures and heating and cooling loads in two steps. In the first step, the loads are calculated assuming a fixed indoor air temperature. In the second step, the fixed temperature solution is used as a basis for finding the floating temperatures within a thermostat dead band and the resulting modified loads. DOE 2.1 uses transfer functions rather than a nodal network to compute the temperatures for each wall surface and the air temperature for each zone [3].

Transfer functions relate the current values of temperatures and heating loads to past values. They are the mechanisms by which DOE 2.1 accounts for the effects of thermal mass in the building. One set of transfer functions (response factors) is used to compute the heat flow rates at the wall surfaces for the fixed indoor air temperature solution. Another set of transfer functions 
(weighting factors) is used to compute the load that must be met to keep the zone temperatures constant given the wall surface heat flow rates. Finally, a set of room air transfer functions and the characteristics of the heating and cooling equipment for a zone are used to calculate the floating air temperature and zone loads. Note that the loads calculated with floating temperatures may differ substantially from the constant temperature loads calculated by the first part of the program. With this solution strategy, DOE 2.1 has eliminated the need for solving simultaneous equations to obtain the various zone air temperatures at each time step, thus allowing for fast (inexpensive) execution of the program. The price for this speed is that the dynamic interaction between zones that are closely coupled and exhibit large temperature variations can only be approximated. Atriums or attached sunspaces are examples of passive solar designs in which this situation can occur.

\subsubsection{Radiation Modeling}

DOE 2.1 can accept solar radiation data from a TMY format tape. It can also calculate solar radiation data using algorithms that consider cloud cover data contained on TRY format or special California data tapes. The solar radiation model for DOE 2.1 calculates the radiation striking the building as a combination of direct beam and diffuse radiation. The diffuse radiation model is anisotropic, concentrating radiation near the sun's position. There is also a diffuse contribution due to radiation reflected from the ground. Ground reflectance is specified as a constant for each wall. Thus, seasonal variations in ground reflectance (snow) are not modeled. DOE 2.1 models the shading of the surface by another only when explicitly directed to do so by the user. Thus, the flexibility exists to model only important shading surfaces, neglecting the effects of minor shading. To find the portion of a surface that is shaded, the surface is divided into a user-specified number of strips. A rectangular shadow area for each strip is then calculated. Shading patterns are computed for each hour one day per month and are used for the entire month. The program is capable of modeling only rectangular surfaces so that some areas can only be approximated for shading calculations. The absorptance of opaque exterior surfaces is a user input.

DOE 2.1 provides three alternatives to determine the amount of radiation that enters the building through the windows. Window properties may be completely specified by the user, windows may be specified as single, double, or triple pane with the glass type selected from a library; or the user may select a default glass and input a shading coefficient. The view factors for diffuse radiation from the sky and diffuse radiation from the ground are user-specified constants. Once the solar radiation has entered a window, the user may specify the distribution of radiation onto each interior surface as a constant. Thus, the hourly and seasonal variation of the distribution is not modeled. The program calculates the amount reflected back out the windows using a simple algorithm that assumes all interior surfaces have the same absorptivity and considers multiple reflections. All reflected radiation is considered to be diffuse.

Linearized infrared radiation exchange between the wall surfaces bounding a zone is considered when generating the custom weighting factors that characterize the zone. Additionally, the infrared reradiation to the sky is estimated as $63 \mathrm{~W} / \mathrm{m}^{2}\left(20 \mathrm{Btu} / \mathrm{h} \mathrm{ft}^{2}\right)$ for an upward-facing horizontal surface assuming a clear sky. This number is then scaled using the cloud cover and the cosine of the wall zenith angle. Reradiation is zero for a vertical wall or for a cloud-covered sky. 
The simplifications in solar radiation distribution within zones and in infrared radiation modeling may be unacceptable to a user simulating a passive solar design.

\subsubsection{Convection and Conduction Modeling}

The film coefficients for interior surfaces in DOE 2.1 are user-specified constants. The program expects these coefficients to represent contributions from both infrared and convective heat transfer. It automatically separates the contributions when generating custom weighting factors. The film coefficients for exterior surfaces are calculated as a function of the user-specified roughness of the surface and the wind speed taken from the weather file. Specifying the film coefficients as independent of surface temperatures and, more importantly, the direction of heat flow, allows the program to solve for the heat flow through the surfaces without using an iterative procedure. Execution time is thus decreased.

Infiltration can be modeled in one of three ways:

- The air changes per hour may be specified for each zone.

- The "crack" method [3] may be employed. Infiltration coefficients are specified for each exterior wall and window, and a neutral point (at which inside-outside pressures are the same) is specified for each zone.

- A "residential" method may be used that calculates the air changes per hour as a function of the wind speed and the outdoor and indoor temperature difference.

Additionally, the calculated infiltration may be scaled on an hourly basis by a user input schedule. Natural advection or scheduled fans between zones are not modeled.

Conduction in DOE 2.1 is handled through the transfer functions. The exterior wall properties are embodied in the response factors; the interior wall properties are embodied in the weighting factors. A user may create walls for a simulation or select walls from the program library. Light walls may appear as pure resistances. To compute the heat exchange with the ground, average ground temperature for each month is input by the user. Any thermal resistance from the ground (i.e., between an exterior wall surface and the ground temperature) must be input as part of the wall specification. The thermal capacitance of the ground is not explicitly considered.

\subsubsection{Systems and Schedules}

In addition to calculating the thermal loads and temperatures for the building, DOE $2.1 \mathrm{can}$ simulate the performance of the heating and cooling equipment in meeting these loads. Thus, the program simulates not only the effects of finite sized equipment, but also the efficiency with which the equipment operates. Among the equipment types that can be simulated are active solar heating devices for space or water heating. DOE 2.1 can also simulate the effects of lighting and furniture on the building energy consumption. It does not contain the capability to explicitly model Trombe walls, rock beds, water walls, phase change materials, daylighting, or thermosiphon air panels.

The program has extensive capability to simulate schedules. Internal gains (such as people, equipment, and lighting) can be individually scheduled. 
Night insulation and shading can be scheduled for each window. Economizer cycles can also be modeled.

The systems and schedules in DOE 2.1 allow most conservation measures to be simulated, but using the program to assess the performance of an aggressive solar design may be difficult.

\subsection{References}

1. Hittle, D.C. 1979. The Buildings Loads Analysis and System Thermodynamics Program, Version 2.0 Users Manual, Vols. I and II. Technical Reports E-153/ADA072272 and ADA0722730, Washington, DC: U.S. Army Construction Engineering Research Laboratory (CERL).

2. Arumi, F.N. et al. 1979. The DEROB III System: Users Manual Vols. I and II. Austin, TX: Solenco.

3. Lawrence Laboratories. 1980. DOE-2 Users Guide Version 2.1, Report \#LBL-8689, Rev. 1, Berkeley, CA: Lawrence Laboratories.

4. Palmiter, L. and Wheeling, T. 1979. "SUNCAT 2.4 User Notes." Butte, MT: Center for Appropriate Technology.

5. Gates. 1964. Energy Exchange in the Biosphere. New York: Harper and Row, 1964.

6. Walton, G. N. 1980. A New Algorithm for Radiant Interchange in Room Loads Calculations. ASHRAE Transactions, Vol. 86, Pt. 2. 


\subsection{Conclusions and Future Work}

Validating building energy analysis simulations (BEAS) is a task that will require a multiyear effort. Ultimately, the validation effort will answer the question "How accurate is this code for this building type and configuration in this climate?" Although this question cannot yet be confidently answered, we have made significant progress in developing a methodology for so doing. An overview of our results is given in Sec. 8.1. Directions for future work are stated in Sec. 8.2.

\subsection{Conclusions}

Our examination of previous work indicates a lack of appropriate data for validation. The existing data base is neither detailed enough to properly quantify important input parameters, nor broad enough to include a sufficient variety of building types, configurations, and locations. Previous validation attempts have not propagated input errors onto output variables to define the total problem uncertainty. Furthermore, little effort has been directed to mechanism-level validation; and, thus, there has been no way to resolve the ambiguity in system-level comparisons caused by compensating errors. We therefore conclude that empirical validation studies should measure key inputs, address error propagation, and isolate those individual mechanisms responsible for model or input uncertainty.

Our comparative studies show that the various codes have uncomfortably large discrepancies, even for a very simple building. To state the source of these discrepancies, we need to define a few terms. A building consists of components (walls, windows, HVAC, ground, etc.) that together form a system driven by the environment and internal loads. Hence, we can divide the general problem into two areas: component treatment, given the boundary conditions, and establishment of boundary conditions. The latter consists of two types: forcing functions, such as sun, internal loads, ambient air, and ground temperature; and internal coupling between components, such as convection, infrared radiation, and reflected solar radiation.

It is clear from our analytical validation work that the simulations correctly handle thin-skin components dominated by the linear solids conduction mechanism, subject to either convective or radiative boundary conditions. (DEROB-3 is an exception here; see Section 5.0) This is no real surprise since all the codes document rigorous approaches to solving the conduction field problem, given the boundary conditions. Since the comparative studies show that large differences in system predictions exist, we conclude that the boundary condition models inherent in BEAS give rise to the bulk of these differences and to internal errors in general.

Uncertainty in external boundary conditions includes (1) sky diffuse irradiance, (2) sky infrared flux, and (3) infiltration rates. Internal boundary conditions that are uncertain are: (1) intrazone convective coupling, (2) interzone air flow, (3) infrared coupling, and (4) solar internal distribution.

Our examination of code documentation indicates several areas of component uncertainty. First, the ground beneath the building is a potentially important component that is poorly treated by all the simulations. It is generally unclear, and code documentation provides little guidance on how to force this inherently multidimensional problem into the one-dimensional framework assumed 
by the simulations. Basic problems or uncertainty concerning water transport and ground thermal conductivity are also barriers to effective ground coupling model development. Second, most of the codes do not consider phase change components despite their potential importance. Third, BEAS predictions of optical behavior of glazings (transmission, absorption in panes) have not been directly validated, although, except for diffuse transmission and edge support effects, it probably is treated adequately by the simulations. Fourth, considerable differences exist in default average window $U$-values and need to be resolved. Fifth, treatment of moisture adsorption/desorption should be incorporated into the codes.

\subsection{Future Work}

SERI's validation methodology has included comparative, analytical, and empirical approaches. In general, we recommend that the empirical Class A validation methodology be refined in four major areas:

- Problem uncertainty. Any empirical validation must include a quantitative definition. Quadrature addition of contributing errors with numerical computation of the relevant partial derivatives should be explored for deriving a quantitative definition of validation.

- Compensating errors. Measurements must be taken at the mechanism level to resolve the effects of compensating errors. Data must be detailed enough to separate skin-load and solar absorbed quantities.

- System performance indices. A few significant validation indicators need to be developed. Standard statistical indices such as RMS differences can be misleading unless appropriately applied.

- Building energy metrology. We need to characterize those key mechanisms that cannot currently be measured adequately. These areas (and probable solutions) include:

- Air flow (multiple tracer gas approaches)

- Total transmitted solar radiation (linearly integrating pyranometer)

- Sky diffuse irradiance (sky radiance mapper)

- Ground coupling (ground conductivity and water content measurements).

The empirical Class A methodology developed here must be systematically applied. It will be necessary to develop software, which automatically performs the key energy balance computations that establish completeness and accuracy of monitoring.

We need to gather and analyze Class A data from additional building types and configurations to establish the parametric range within which the BEAS are reliable. This should include, but not be limited to, sunspaces, thermal storage walls, advectively coupled source and sink zones, and natural cooling systems.

The work completed to date has focused on envelope load-dominated buildings. It is a logical next step to extend the monitoring and validation methodologies to include larger internally loaddominated buildings including the associated heating, ventilating, and air conditioning equipment commonly used in these buildings. 
A methodology needs to be developed that will allow a systematic prediction of the probable error to be associated with a given simulation problem caused by either external or internal uncertainties. Although present approaches can quantify the error for a given system in a given climate, it is important to be able to predict errors for any configuration in any climate. Such a methodology would probably be centered around the impact of boundary condition uncertainty. 


\section{Appendix A}

The analytical verification procedure consists of several tests, each of which verifies a combination of mechanisms in a code. These tests were chosen because they verify the algorithms for the mechanisms that are most important in predicting the thermal performance of buildings. Each test consists of a building description, an analytical model of the heat transfer characteristics of the building, a weather file, and a set of expected results for the test.

The tests can be divided into two groups: Sections A.1 through A.6 contain tests that do not use mechanisms involving solar radiation; Sections A.7 through A.10 contains those that do.

The first group of tests used the building model A; and the second, model B (see below).

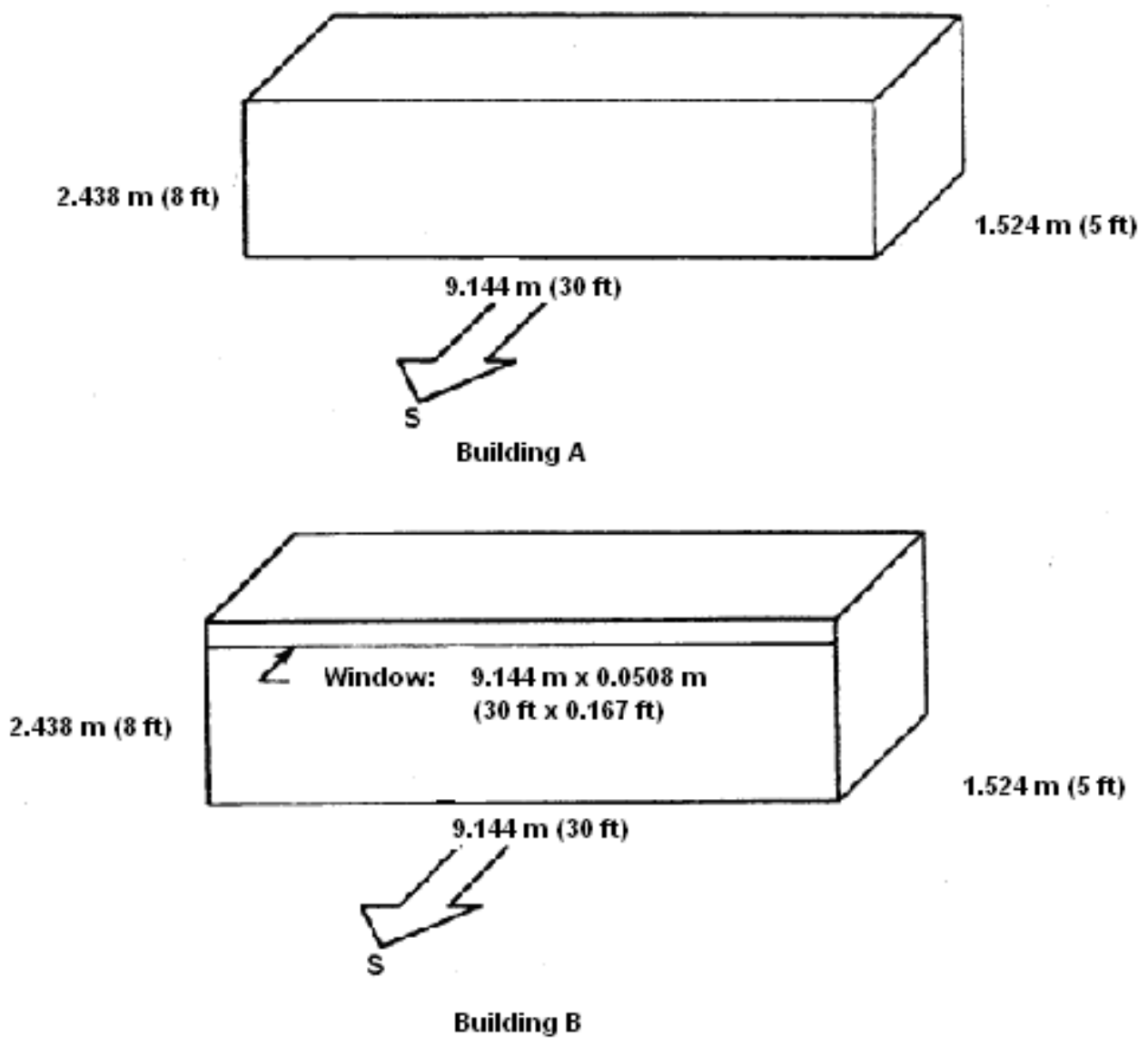

\section{A.1 Low-Mass Decay and Steady-State Heat Loss Test}

\section{Mechanisms Tested}

Steady-state heating and cooling loads

Wall conductivity

Response of internal temperature to changes in external temperature 


\section{Weather Type}

A

Test Building

A

\section{Test Building Description}

No windows

No infiltration

No ground coupling

Zero external absorptivity/emissivity

Zero internal absorptivity/emissivity

Single zone

Building is at sea level

Zone air is massless

Wall characteristics:

- Inside film coefficient $=5.6786 \mathrm{~W} / \mathrm{m}^{2}{ }^{\circ} \mathrm{C}\left(1 \mathrm{Btu} / \mathrm{h} \mathrm{ft}^{2}{ }^{\circ} \mathrm{F}\right)$

0.0127-m (0.5-in.) gypsum board $\left(\mathrm{k}=0.4327 \mathrm{~W} / \mathrm{m}^{\circ} \mathrm{C}\left(0.25 \mathrm{Btu} / \mathrm{h} \mathrm{ft}{ }^{\circ} \mathrm{F}\right)\right.$

$\rho=1249 \mathrm{~kg} / \mathrm{m}^{3}\left(78 \mathrm{lb} / \mathrm{ft}^{3}\right), \mathrm{C}_{\mathrm{p}}=1.089 \mathrm{~kJ} / \mathrm{kg}{ }^{\circ} \mathrm{C}\left(0.26 \mathrm{Btu} / \mathrm{lb}{ }^{\circ} \mathrm{F}\right)$

- Zero mass insulation with an R-value of $2.25 \mathrm{~m}^{2}{ }^{\circ} \mathrm{C} / \mathrm{W}\left(12.783 \mathrm{Btu} / \mathrm{h} \mathrm{ft}{ }^{\circ} \mathrm{F}\right)$

- $\quad$ Outside film coefficient $=22.7 \mathrm{~W} / \mathrm{m}^{2}{ }^{\circ} \mathrm{C}\left(4 \mathrm{Btu} / \mathrm{h} \mathrm{ft}{ }^{\circ}{ }^{\circ} \mathrm{F}\right)$

Note: This wall section applies to south, east, north, and west walls and ceiling

$$
\begin{array}{lll}
\text { Total wall area } & =65.96 \mathrm{~m}^{2}\left(710 \mathrm{ft}^{2}\right) \\
\text { Wall U-value } & =0.4 \mathrm{~W} / \mathrm{m}^{2}{ }^{\circ} \mathrm{C}\left(0.0704 \mathrm{Btu} / \mathrm{h}{ }^{\circ} \mathrm{F}\right) \\
\text { Wall R-value } & =2.5 \mathrm{~m}^{\circ} \mathrm{C} / \mathrm{W}\left(14.2 \mathrm{~h}{ }^{\circ} \mathrm{F} / \mathrm{Btu}\right) \\
\text { Building UA } & =26.375 \mathrm{~W} /{ }^{\circ} \mathrm{C}\left(50 \mathrm{Btu} / \mathrm{h}{ }^{\circ} \mathrm{F}\right) \\
\text { Heating set point } & =26.67^{\circ} \mathrm{C}\left(80^{\circ} \mathrm{F}\right) \\
\text { Cooling set point } & =-6.67^{\circ} \mathrm{C}\left(20^{\circ} \mathrm{F}\right)
\end{array}
$$

\section{Procedure}

Run this test with Type A weather from January through July.

\section{Interpretation of Results}

\section{Steady-State Tests}

The building steady-state heating load is read as the auxiliary heating load in the output of the program being tested after this load has reached a steady-state value in January.

\section{Setting}

$\mathrm{q}^{\cdot}$ auxiliary heating load $=\mathrm{UA}\left(\mathrm{T}_{\text {inside }}-\mathrm{T}_{\text {outside }}\right)$, and $\mathrm{T}_{\text {inside }}-\mathrm{T}_{\text {outside }}=-6.67^{\circ} \mathrm{C}-\left(-28.89^{\circ} \mathrm{C}\right)=$ $22.22^{\circ} \mathrm{C}\left(40^{\circ} \mathrm{F}\right) \mathrm{UA}=\mathrm{q}^{\cdot} / 22.22^{\circ} \mathrm{C}\left(\mathrm{q}^{\circ} / 40^{\circ} \mathrm{F}\right)$ 
Table A1. Normalized Low-Mass Temperature Decay

\begin{tabular}{|c|c|}
\hline $\mathbf{t}(\mathbf{h r})$ & $\mathbf{T}_{\mathbf{N A}}{ }^{(\tau)}$ \\
\hline 0 & 1.0 \\
\hline 1 & 0.914 \\
\hline 2 & 0.835 \\
\hline 3 & 0.763 \\
\hline 4 & 0.697 \\
\hline 5 & 0.637 \\
\hline 6 & 0.582 \\
\hline 7 & 0.532 \\
\hline 8 & 0.486 \\
\hline
\end{tabular}

The building UA should be $26.375 \mathrm{~W} /{ }^{\circ} \mathrm{C}$. If it differs from this value, and the building model and weather data have been correctly input, something is wrong with the simulation model or the solution technique.

A similar result can be found for the steady-state cooling load using the steady-state auxiliary cooling load in April.

\section{Dynamic Tests}

The dynamic tests use the hourly temperature profiles for March (temperature rise) and June (temperature decay). The hourly temperatures, when normalized, should agree with those values in Table A-I. The procedure for normalization and check of the output data for temperature rise is as follows:

- To ensure that the exponential nature of the temperature decay is fully developed, use $\mathrm{T}_{\mathrm{wo}}$, the initial wall temperature (or inside wall surface temperature) for hour 3 on March 1.

- Calculate $T_{N}(t)=\frac{T_{w}(t)-T_{\infty}}{T_{w o}-T_{\infty}}, 0<t \leq 8 \mathrm{hr}$

Where,

$\begin{array}{lll}\mathrm{T}_{\mathrm{N}}(\mathrm{t}) & = & \text { normalized temperature } \\ \mathrm{T}_{\mathrm{w}} & = & \text { room air temperature (inside wall surface temperature) } \\ \mathrm{T}_{\mathrm{oo}} & = & \text { ambient temperature }\left(48.89^{\circ} \mathrm{C}\right) \\ \mathrm{T}_{\mathrm{wo}} & = & \text { initial room air temperature }(\text { defined earlier) } \\ \mathrm{t} & = & \text { time after } \mathrm{T}_{\mathrm{wo}} \text { is set. }\end{array}$

Calculate: $T_{N A}(t)=\exp \left[\left(-U_{o} / C_{w}\right) t\right], 0<t \leq 8 h r$

Where,

$$
\begin{array}{rll}
\mathrm{T}_{\mathrm{NA}}(\mathrm{t}) & = & \text { normalized temperature } \\
\mathrm{U}_{\mathrm{o}} & = & \left(0.5 \mathrm{R}_{\text {gyp }}+\mathrm{R}_{\text {ins }}+\mathrm{R}_{\text {ofc }}\right)-1 \\
\mathrm{R}_{\text {gyp }} & = & \text { R-value of gypsum board } \\
& = & (0.0127 \mathrm{~m}) /\left(0.4327 \mathrm{~W} / \mathrm{m}^{\circ} \mathrm{C}\right) \\
& = & 0.0294 \mathrm{~m}^{2}{ }^{\circ} \mathrm{C} / \mathrm{W}\left(0.167 \mathrm{~h} \mathrm{ft}^{2}{ }^{\circ} \mathrm{F} / \mathrm{Btu}\right)
\end{array}
$$




$$
\begin{aligned}
\mathrm{R}_{\text {ins }}=\quad & \text { R-value of insulation }=2.25 \mathrm{~m}^{2}{ }^{\circ} \mathrm{C} / \mathrm{W} \\
\mathrm{R}_{\text {ofc }}=\quad & \left(12.78^{3} \mathrm{~h} \mathrm{ft}^{2}{ }^{\circ} \mathrm{F} / \text { Btu }\right) \\
& \begin{array}{l}
\text { R-value of exterior air film }=0.044 \mathrm{~W} / \mathrm{m}^{2}{ }^{\circ} \mathrm{C} \\
\left(0.25 \mathrm{~h} \mathrm{ft}^{2}{ }^{\circ} \mathrm{F} / \text { Btu }\right)
\end{array}
\end{aligned}
$$

Therefore,

$$
\begin{array}{rll}
\mathrm{U}_{\mathrm{o}} & = & {[0.5(0.0294)+2.25+0.044]^{-1} \mathrm{~W} / \mathrm{m}^{2}{ }^{\circ} \mathrm{C}} \\
& = & 0.433 \mathrm{~W} / \mathrm{m}^{2}{ }^{\circ} \mathrm{C} \\
\mathrm{C}_{\mathrm{w}} & = & \text { thermal capacity of a unit area of wall } \\
& = & (0.0127 \mathrm{~m})\left(1249 \mathrm{~kg} / \mathrm{m}^{3}\right)\left(1089 \mathrm{~J} / \mathrm{kg}^{\circ} \mathrm{C}\right) \\
& =17,274 \mathrm{~J} / \mathrm{m}^{2}{ }^{\circ} \mathrm{C} \\
\mathrm{T}_{\mathrm{NA}}(\mathrm{t}) & = & \exp -\left(0.433 \mathrm{~J} / \mathrm{m}^{2}{ }^{\circ} \mathrm{C}\right) /\left(17,274 \mathrm{~J} / \mathrm{m}^{2}{ }^{\circ} \mathrm{C}\right) \\
& = & \mathrm{e}-(0.000251 / \mathrm{s}) \tau={ }_{\mathrm{e}}-(0.0902 / \mathrm{h}) \tau
\end{array}
$$

These values are presented in Table A-1 and Figure A-1.

Compare $\mathrm{Tn}(\mathrm{t})$ and $\mathrm{T}_{\mathrm{NA}}(\mathrm{t})$ for each $\mathrm{t}$.

Quantitative methods of comparing these values have not been developed. However, extreme differences, such as in the DEROB-3 results indicate a problem with the program being tested. Note also that the convergence limits in a code using an iterative solution technique should be made extremely narrow for this test. Otherwise, an apparent discrepancy could result between the output of the code and the analytical solution.

The normalized temperature values for the temperature decay (June) should be identical to the temperature rise. To calculate these values, the same procedure is used, except that $\mathrm{T}=\infty-$ $28.89^{\circ} \mathrm{C}\left(-20^{\circ} \mathrm{F}\right)$.

Selected codes may place limits on the choices of thermal mass $\left(\mathrm{C}_{\mathrm{w}}\right)$ and heat transfer coefficients $\left(U_{0}\right)$. If this is true, then normalized values of $T_{N A}(t)$ must be calculated with the values of $\mathrm{C}_{\mathrm{w}}$ and $\mathrm{U}_{\mathrm{o}}$ used in each code. The same procedure is used to evaluate the results with a possible increase in the time parameter $\tau$ for more massive or better insulated buildings. The temperature profiles for the March and June test periods can also be compared directly to the infinite series solution presented in Table A-2 and Figure A-1.

\section{Notes on Implementation of the Test}

This test is the basis for several others, and its accuracy will be based on careful input development and possible code modifications. Mechanisms that do not conform to the boundary and initial conditions of the analytical solution must be eliminated or changed. In the codes we have tested, these mechanisms include internal infrared radiation networks, variable film coefficients, insulation with mass, and others. Additionally, if the weather processors will not accept synthetic and unusual weather data files, they will have to be changed so that the test can be run. 


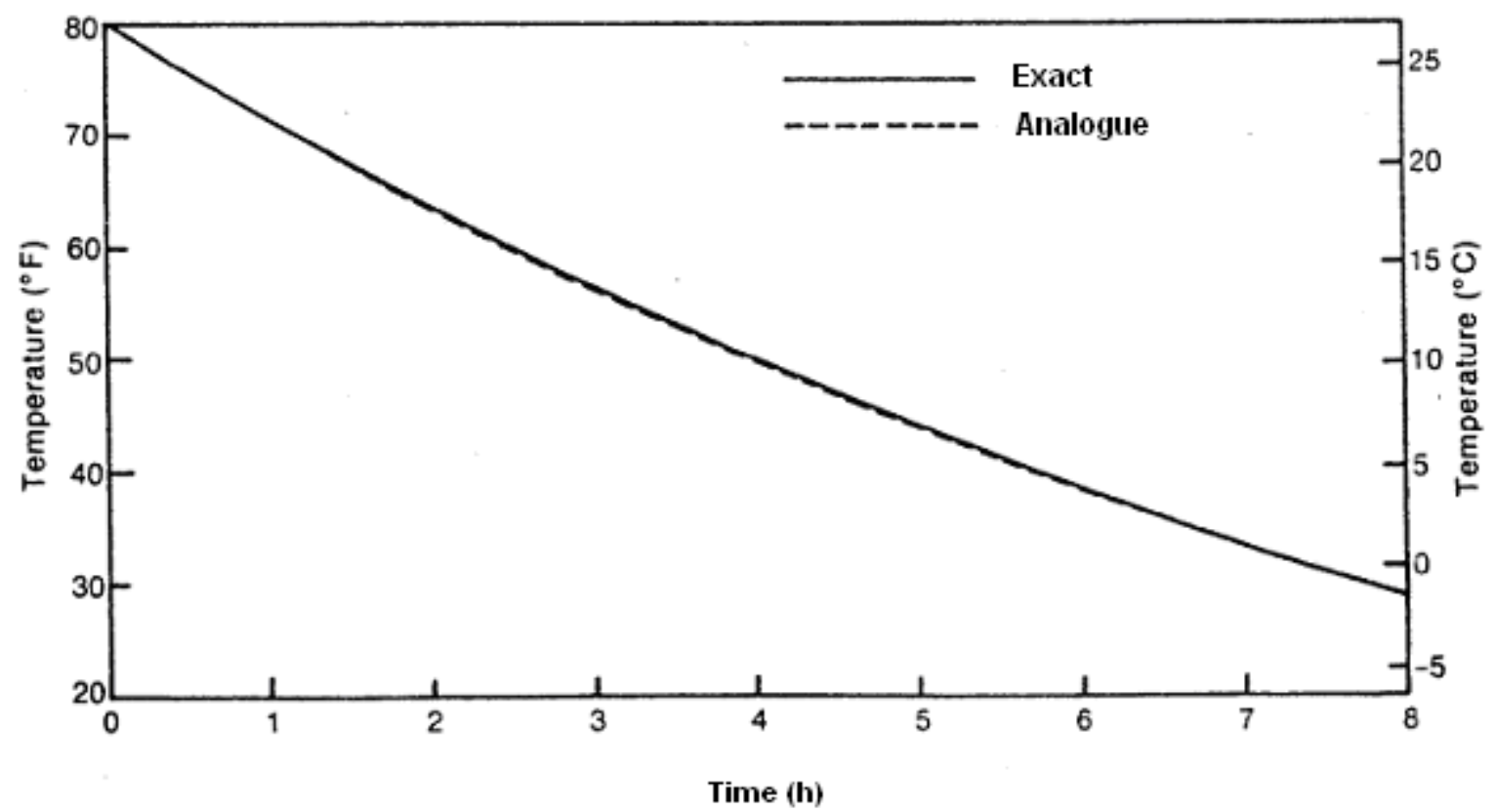

Figure A-1. Low-mass decay solutions

Table A-2. Exact Solution, Low-Mass Temperature Rise and Decay

\begin{tabular}{|c|c|c|c|c|}
\hline \multirow{2}{*}{$\mathbf{T}(\mathbf{h})$} & \multicolumn{2}{|c|}{$\mathbf{T}_{\text {rise }}$} & \multicolumn{2}{|c|}{$\mathbf{T}_{\text {decay }}$} \\
\cline { 2 - 5 } & $\left.\mathbf{(}{ }^{\circ} \mathbf{C}\right)$ & $\left({ }^{\circ} \mathbf{F}\right)$ & $\left({ }^{\circ} \mathbf{C}\right)$ & 80.0 \\
\hline 0 & -6.7 & 20.0 & 26.7 & 71.6 \\
\hline 1 & -2.0 & 28.4 & 22.0 & 63.6 \\
\hline 2 & 2.4 & 36.4 & 17.6 & 56.4 \\
\hline 3 & 6.4 & 43.6 & 13.6 & 49.8 \\
\hline 4 & 10.1 & 50.2 & 9.9 & 43.8 \\
\hline 5 & 13.4 & 56.2 & 6.6 & 38.3 \\
\hline 6 & 16.5 & 61.7 & 3.5 & 33.2 \\
\hline 7 & 19.3 & 66.8 & 0.7 & 28.6 \\
\hline 8 & 21.9 & 71.4 & -1.9 & \\
\hline
\end{tabular}

\section{A.2 Mid-Mass Decay and Steady-State Heat Loss Test}

\section{Mechanisms Tested}

The effects of a change of thermal mass on the mechanisms tested in Section A.1.

\section{Weather Type}

A

\section{Test Building}

A

\section{Test Building Description}

Same as in Section A.I except 
Wall characteristics:

- $\quad$ Inside film coefficient $=5.6786 \mathrm{~W} / \mathrm{m}^{2}{ }^{\circ} \mathrm{C}\left(1 \mathrm{Btu} / \mathrm{h} \mathrm{ft}^{2}{ }^{\circ} \mathrm{F}\right)$

- $\quad 0.016-\mathrm{m}(4-i n$.$) concrete \left[\mathrm{k}=0.935 \mathrm{~W} / \mathrm{m}^{\circ} \mathrm{C}\left(0.54 \mathrm{Btu} / \mathrm{h} \mathrm{ft}^{2}{ }^{\circ} \mathrm{F}\right)\right.$ $\rho=2307 \mathrm{~kg} / \mathrm{m}^{3}\left(144 \mathrm{lb} / \mathrm{ft}^{3}\right), \mathrm{C}_{\mathrm{p}}=0.67 \mathrm{~kJ} / \mathrm{kg}{ }^{\circ} \mathrm{C}\left(0.16 \mathrm{Btu} / \mathrm{lb}{ }^{\circ} \mathrm{F}\right)$

- Zero mass insulation with an R-value of $2.172 \mathrm{~m}^{2}{ }^{\circ} \mathrm{C} / \mathrm{W}\left(12.332 \mathrm{~h} \mathrm{ft}{ }^{\circ} \mathrm{F} / \mathrm{Btu}\right)$

- $\quad$ Outside fi $1 \mathrm{~m}$ coefficient $=22.7 \mathrm{~W} / \mathrm{m}^{2}{ }^{\circ} \mathrm{C}\left(4 \mathrm{Btu} / \mathrm{h} \mathrm{ft}^{2}{ }^{\circ} \mathrm{F}\right)$

Note: The thickness of the insulation has changed so that the overall U- value of the wall remains the same as in Section A.1.

\section{Procedure}

Same as in Section A.1.

\section{Interpretation of Results}

\section{Steady-State Tests}

Same as in Section A.1.

\section{Dynamic Tests}

The procedure is the same as in Section A.1, except that the values of some parameters have changed. In particular, the value of $\tau$ for which $T_{w o}$ is chosen and the $C_{w}$ and $U_{o}$ ) are different: $\mathrm{T}_{\mathrm{wo}}$ is set as the zone air temperature at eight hours after the temperature rise test period has begun, and is measured from $\tau=0$ at this time.

Where,

$\begin{array}{lll}\mathrm{U}_{\mathrm{o}} & = & \left(0.5 \mathrm{R}_{\text {concrete }}+\mathrm{R}_{\text {ins }}+\mathrm{R}_{\text {off }}\right)^{-1} \\ \mathrm{R}_{\text {concrete }} & = & 0.1016 \mathrm{~m} / 0.935 \mathrm{~W} / \mathrm{m}^{2}{ }^{\circ} \mathrm{C}=0.1087 \mathrm{~W} / \mathrm{m}^{2}{ }^{\circ} \mathrm{C} \\ \mathrm{R}_{\text {ins }} & = & 2.172 \mathrm{~m}^{2}{ }^{\circ} \mathrm{C} / \mathrm{W}\left(12.332 \mathrm{~h} \mathrm{ft}^{\circ}{ }^{\circ} \mathrm{F} / \mathrm{Btu}\right) \\ \mathrm{R}_{\text {ofc }} & = & 0.044 \mathrm{~m}^{2} \mathrm{C} / \mathrm{W}\left(0.25 \mathrm{~h} \mathrm{ft}{ }^{\circ} \mathrm{F} / \mathrm{Btu}\right)\end{array}$

Therefore,

$$
\begin{array}{lll}
\mathrm{U}_{\mathrm{o}} & = & {[0.5(0.1087)+2.172+0.044]^{-1} \mathrm{~W} / \mathrm{m}^{2}{ }^{\circ} \mathrm{C}} \\
& = & 0.44 \mathrm{~W} / \mathrm{m}^{\circ} \mathrm{C} \\
\mathrm{C}_{\mathrm{w}} & = & (0.1016 \mathrm{~m})\left(2307 \mathrm{~kg} / \mathrm{m}^{3}\right)\left(0.67 \mathrm{~kJ} / \mathrm{kg}{ }^{\circ} \mathrm{C}\right) \\
& = & 157,042 \mathrm{~J} / \mathrm{m}^{2}{ }^{\circ} \mathrm{C} \\
\mathrm{T}_{\mathrm{NA}}(\tau) & \left.=\exp -\left[\left(0.44 \mathrm{~J} / \mathrm{s} \mathrm{m}^{2}{ }^{\circ} \mathrm{C}\right) / 157,042 \mathrm{~J} / \mathrm{m}^{2}{ }^{\circ} \mathrm{C}\right)\right] \tau \\
& & \exp -(0.00000280 / \mathrm{s}) \tau=\exp -(0.0101 / \mathrm{h}) \tau / .
\end{array}
$$

These values are presented in Table A-3 and Figure A.2. The exact solution values are presented in Table A-4 and Figure A-2. 


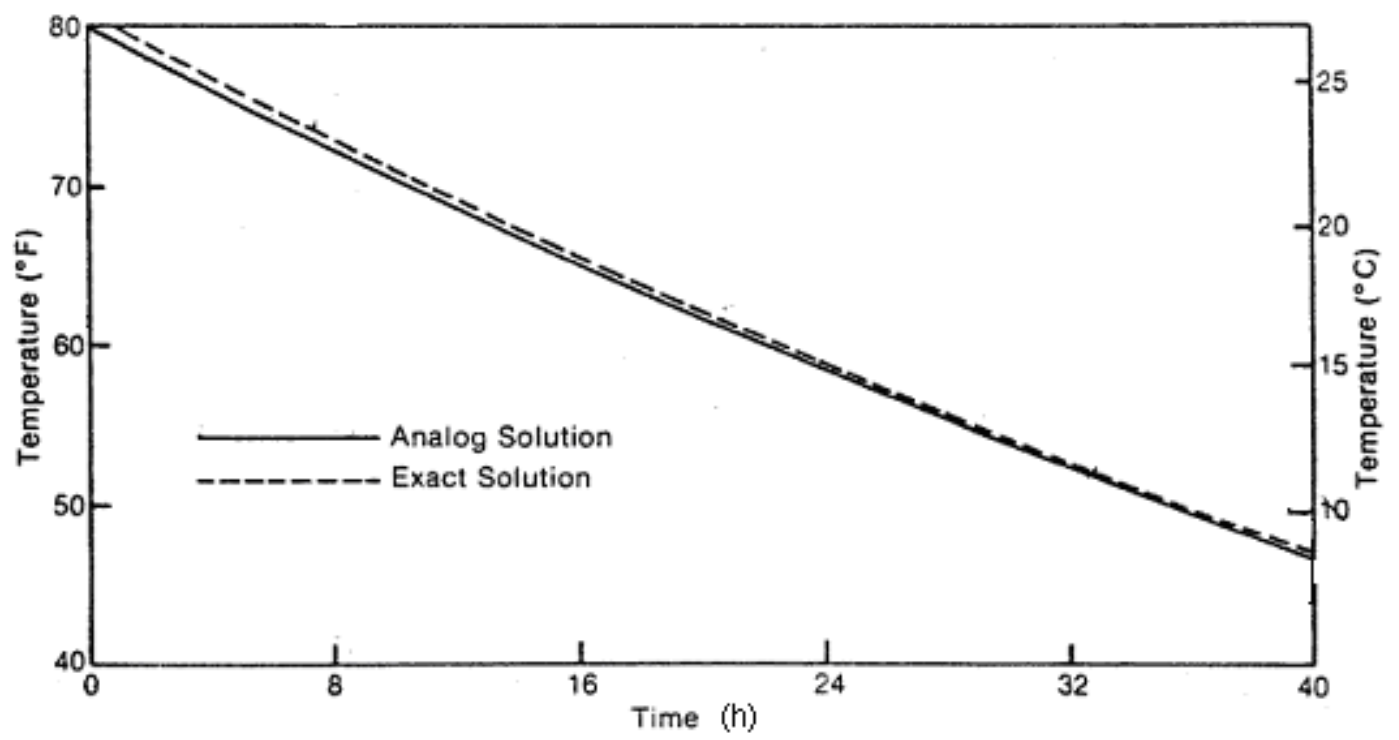

Figure A-2. Mid-mass decay test solutions

Table A-3. Normalized Mid-Mass Temperature Decay

\begin{tabular}{|c|c|}
\hline $\mathbf{t}(\mathbf{h r})$ & $\mathbf{T}_{\mathbf{N A}}(\mathbf{t})$ \\
\hline 0 & 1.0 \\
\hline 4 & 0.960 \\
\hline 8 & 0.922 \\
\hline 12 & 0.886 \\
\hline 16 & 0.851 \\
\hline 20 & 0.817 \\
\hline 24 & 0.785 \\
\hline 28 & 0.754 \\
\hline 32 & 0.724 \\
\hline 40 & 0.695 \\
\hline & 0.668 \\
\hline
\end{tabular}

Table A-4. Exact Solution Mid-Mass Temperature Rise and Decay

\begin{tabular}{|c|c|c|c|c|}
\hline $\mathbf{t}(\mathbf{h r})$ & \multicolumn{2}{|c|}{$\mathbf{T}_{\text {rise }}$} & \multicolumn{2}{c|}{$\mathbf{T}_{\text {decay }}$} \\
\hline & $\left({ }^{\mathbf{C}} \mathbf{C}\right)$ & $\left({ }^{\circ} \mathbf{F}\right)$ & $\left({ }^{\circ} \mathbf{C}\right)$ & $\left({ }^{\circ} \mathbf{F}\right)$ \\
\hline 0 & -6.67 & 20.0 & 26.67 & 80.0 \\
\hline 4 & -4.89 & 23.2 & 24.89 & 76.8 \\
\hline 8 & -2.72 & 27.1 & 22.72 & 72.9 \\
\hline 12 & -0.67 & 30.8 & 20.67 & 69.2 \\
\hline 16 & 1.33 & 34.4 & 18.67 & 65.6 \\
\hline 20 & 7.22 & 27.8 & 16.78 & 62.2 \\
\hline 24 & 5.06 & 41.1 & 14.94 & 58.9 \\
\hline 28 & 6.78 & 44.2 & 13.22 & 55.8 \\
\hline 32 & 8.44 & 47.2 & 11.56 & 52.8 \\
\hline 36 & 10.06 & 50.1 & 9.94 & 49.9 \\
\hline 40 & 11.61 & 52.9 & 8.39 & 47.1 \\
\hline
\end{tabular}


Notes on Implementation of the Test

See Section A.1.

\section{A.3 High-Mass Decay and Steady-State Beat Loss Test}

\section{Mechanisms Tested}

The effects of a change of thermal mass on the mechanisms tested in Section A.1.

\section{Weather Type}

A

\section{Test Building}

A

\section{Test Building Description}

Same as in Sec. A.1 except

Wall characteristics:

- Inside film coefficient $=5.6786 \mathrm{~W} / \mathrm{m}^{2}{ }^{\circ} \mathrm{C}\left(1 \mathrm{Btu} / \mathrm{h} \mathrm{ft}{ }^{\circ}{ }^{\circ} \mathrm{F}\right)$

- 0.1778 -m (7-in.) concrete

$$
\begin{aligned}
& {\left[\mathrm{k}=0.935 \mathrm{w} / \mathrm{m}^{2}{ }^{\circ} \mathrm{C}\left(0.54 \mathrm{Btu} / \mathrm{h} \mathrm{ft}{ }^{\circ} \mathrm{F}\right),\right.} \\
& \rho=2307 \mathrm{~kg} / \mathrm{m}^{3}\left(144 \mathrm{lb} / \mathrm{ft}^{3}\right), \\
& \left.c_{\mathrm{p}}=0.67 \mathrm{~kJ} / \mathrm{kg}^{\circ} \mathrm{C}\left(0.16 \mathrm{Btu} / \mathrm{lb}{ }^{\circ} \mathrm{F}\right)\right]
\end{aligned}
$$

- Zero mass insulation with an R-value of $2.091 \mathrm{~m}^{2}\left(11.872 \mathrm{~h} \mathrm{ft}^{2}{ }^{\circ} \mathrm{F} / \mathrm{Btu}\right)$

- Outside film coefficient $=22.7 \mathrm{~W} / \mathrm{m}^{2}{ }^{\circ} \mathrm{C}\left(4 \mathrm{Btu} / \mathrm{h} \mathrm{ft}^{2}{ }^{\circ} \mathrm{F}\right)$

Note: The thickness of the insulation has changed so that the overall U-value of the wall remains the same as in Section A.l.

\section{Procedure}

Same as in Section A.1.

\section{Interpretation of Results}

\section{Steady-State Tests}

The same as in Section A.1.

\section{Dynamic Tests}

The procedure is the same as in Section A.l, except that the values of some parameters have changed. In particular, the value of $\tau$ for which $T_{w o}$ is chosen and the $C_{w}$ and $U_{o}$ are different: $\mathrm{T}_{\mathrm{wo}}$ is set as the zone air temperature at 30 hours, after the temperature rise test period has begun (hour 6 on March 2), and $\tau$ is measured from $\tau=0$ at this time. 
Where,

$\begin{array}{lll}\mathrm{U}_{\mathrm{o}} & = & \left(0.5 \mathrm{R}_{\text {concrete }}+\mathrm{R}_{\text {ins }}+\mathrm{R}_{\text {ofc }}\right)^{-1} \\ \mathrm{R}_{\text {concrete }} & = & (0.1778 \mathrm{~m}) /\left(0.935 \mathrm{~W} / \mathrm{m}^{\circ} \mathrm{C}\right)=0.1902 \mathrm{~m}^{2}{ }^{\circ} \mathrm{C} / \mathrm{W} \\ & & \left(1.08 \mathrm{~h} \mathrm{ft}^{2}{ }^{\circ} \mathrm{F} / \mathrm{Btu}\right) \\ \mathrm{R}_{\text {ins }} & = & 2.091 \mathrm{~m}^{2}{ }^{\circ} \mathrm{C} / \mathrm{W}\left(11.872 \mathrm{~h} \mathrm{ft}^{2}{ }^{\circ} \mathrm{F} / \mathrm{Btu}\right) \\ \mathrm{R}_{\text {ofc }} & = & 0.044 \mathrm{~m}^{2}{ }^{\circ} \mathrm{C} / \mathrm{W}\left(0.25 \mathrm{~h} \mathrm{ft}^{2}{ }^{\circ} \mathrm{F} / \mathrm{Btu}\right) / .\end{array}$

Therefore,

$$
\begin{array}{lll}
\mathrm{U}_{\mathrm{o}} & = & {[0.5(0.1902)+2.091+0.044)^{-1} \mathrm{~W} / \mathrm{m}^{2}{ }^{\circ} \mathrm{C}} \\
& = & 0.448 \mathrm{~W} / \mathrm{m}^{2}{ }^{\circ} \mathrm{C} \\
\mathrm{C}_{\mathrm{w}} & = & (0.1778 \mathrm{~m})\left(2307 \mathrm{~kg} / \mathrm{m}^{3}\right)\left(0.67 \mathrm{~kJ} / \mathrm{kg}{ }^{\circ} \mathrm{C}\right) \\
& =274,824 \mathrm{~J} / \mathrm{m}^{2}{ }^{\circ} \mathrm{C} \\
\mathrm{T}_{\mathrm{NA}}(\mathrm{t}) & \exp -\left[\left(0.448 \mathrm{~W} / \mathrm{m}^{2}{ }^{\circ} \mathrm{C}\right) /\left(274,824 \mathrm{~J} / \mathrm{m}^{2}{ }^{\circ} \mathrm{C}\right)\right] \tau \\
& =\exp -(0.00000163 / \mathrm{s}) \tau=\exp -(0.00587 / \mathrm{h}) \tau / .
\end{array}
$$

These values are presented in Table A-5 and Figure A-3. The exact solution values are presented in Table A-6 and Figure A-3.

Table A-5. Normalized High-Mass Temperature Decay

\begin{tabular}{|c|c|}
\hline $\mathbf{t}(\mathbf{h r})$ & $\mathbf{T}_{\mathbf{N A}}(\mathbf{t})$ \\
\hline 0 & 1.0 \\
\hline 10 & 0.942 \\
\hline 20 & 0.889 \\
\hline 30 & 0.838 \\
\hline 40 & 0.791 \\
\hline 50 & 0.745 \\
\hline 60 & 0.703 \\
\hline 70 & 0.663 \\
\hline 80 & 0.625 \\
\hline
\end{tabular}

Table A-6. Exact Solution, High-Mass Temperature Rise and Decay

\begin{tabular}{|c|c|c|c|c|}
\hline \multirow{2}{*}{$\mathbf{t}(\mathbf{h r})$} & \multicolumn{2}{|c|}{$\mathbf{T}_{\text {rise }}$} & \multicolumn{2}{|c|}{$\mathbf{T}_{\text {decay }}$} \\
\cline { 2 - 5 } & $\left({ }^{\circ} \mathbf{C}\right)$ & $\left({ }^{\circ} \mathbf{F}\right)$ & $\left({ }^{\circ} \mathbf{C}\right)$ & 80.0 \\
\hline 0 & -6.67 & 20.0 & 26.67 & 75.6 \\
\hline 10 & -4.22 & 24.4 & 24.42 & 70.0 \\
\hline 20 & -1.11 & 30.0 & 21.11 & 64.8 \\
\hline 30 & 1.78 & 35.2 & 17.22 & 59.9 \\
\hline 40 & 4.50 & 40.1 & 15.50 & 55.3 \\
\hline 50 & 7.06 & 44.7 & 12.94 & 50.9 \\
\hline 60 & 9.50 & 49.1 & 10.50 & 46.8 \\
\hline 70 & 11.78 & 53.2 & 7.22 & 43.0 \\
\hline 80 & 13.89 & 57.0 & 6.11 & \\
\hline
\end{tabular}




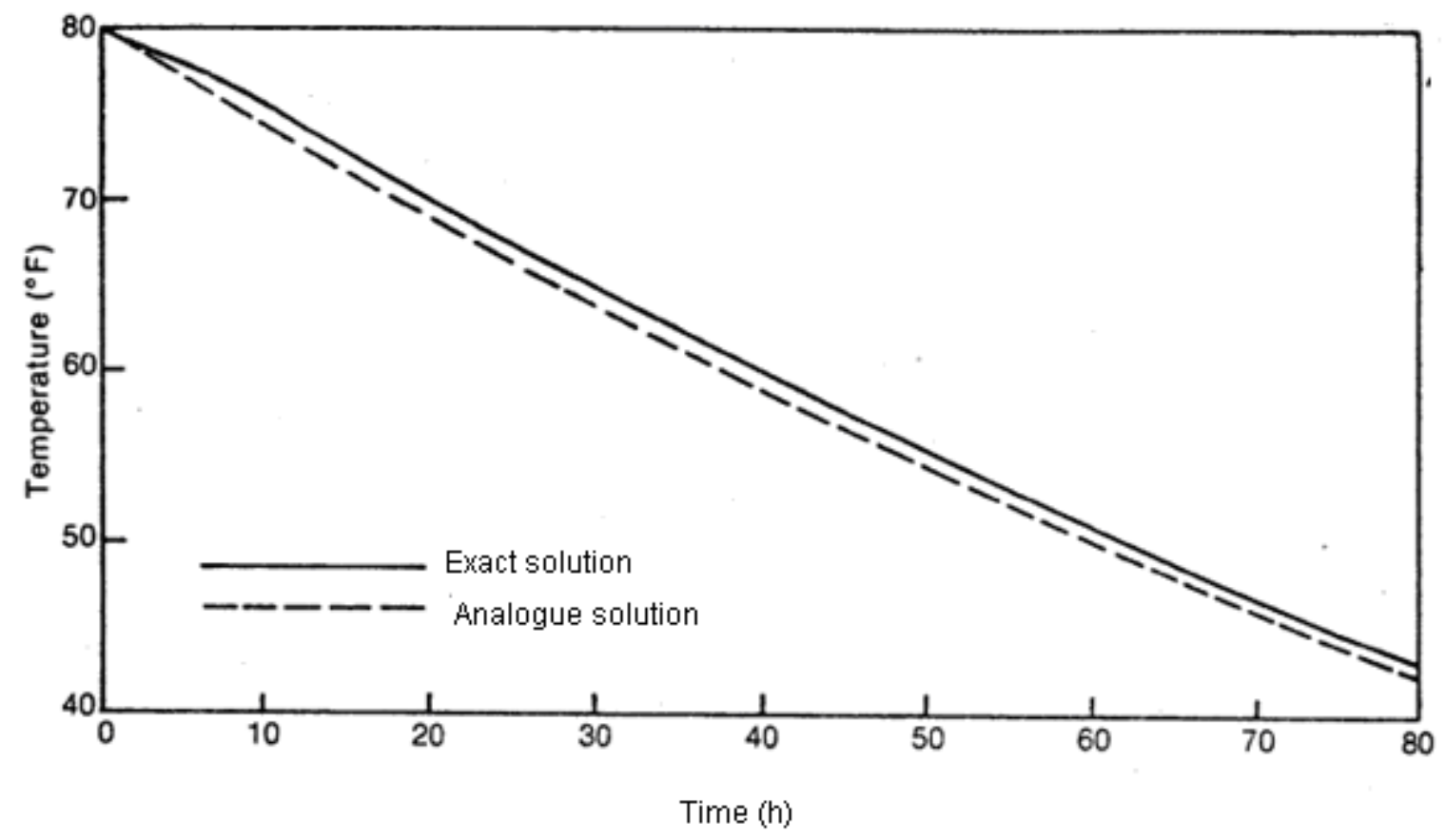

Figure A-3. High-mass decay test solutions

Notes on Implementation of the Test

See Sec. A-I.

\section{A.4 Glazing Test}

\section{Mechanisms Tested}

U-value of glazing, including film coefficients, for single-, double-, and triple-pane windows. This test is a check on the assumptions involving glazing U-values in a code.

\section{Weather Type}

A

\section{Test Building}

\section{A}

\section{Test Building Description}

Same as in Section A.1 except

$0.465-\mathrm{m}^{2}\left(5-\mathrm{ft}^{2}\right)$ window in south wall

Wall area (less window) $=65.5 \mathrm{~m}^{2}\left(705 \mathrm{ft}^{2}\right)$

Window: Normal window glass

Pane thickness

Extinction coefficient Index of refraction

Air gap

$$
\begin{array}{ll}
= & 3.18 \mathrm{~mm}(0.125 \text { in. }) \\
= & 0.197 / \mathrm{mm}(0.5 / \text { in. }) \\
= & 1.526 \\
= & 6.35 \mathrm{~mm}(0.25 \text { in. })
\end{array}
$$


Diffuse angle of incidence $\quad=\quad 60 \mathrm{deg}$

Single, double, or triple glazed

\section{Procedure}

Run this test three times, once for each single, double, and triple glazing. The test is run using the January Type A weather only.

\section{Interpretation of Results}

This test is used to calculate the steady-state U-value of the glazings by subtracting the UA for the walls from the overall UA value of the entire building.

Where,

$$
\begin{aligned}
& (\mathrm{UA})_{\text {window }}=(\mathrm{UA})_{\text {building }}-(\mathrm{UA})_{\text {walls }} \\
& (\mathrm{UA})_{\text {building }}=\dot{q} /\left(\mathrm{T}_{\text {inside }}-\mathrm{T}_{\text {outside }}\right),
\end{aligned}
$$

$\begin{array}{lll}\dot{q} & = & \text { auxiliary heating load from computer output } \\ \mathrm{T}^{\text {inside }} & = & -6.67^{\circ} \mathrm{C}\left(20^{\circ} \mathrm{F}\right) \\ \mathrm{T}_{\text {outside }} & = & -28.89^{\circ} \mathrm{C}\left(-20^{\circ} \mathrm{F}\right) \\ \text { (UA }_{\text {building }} & = & \dot{q} / 22.2^{\circ} \mathrm{CC}\left(\dot{q} / 40^{\circ} \mathrm{F}\right)\end{array}$

and

where

$$
(\mathrm{UA})_{\mathrm{walls}}=\left(\mathrm{U}_{\mathrm{walls}}\right)\left(\mathrm{A}_{\mathrm{walls}}\right)
$$

$$
\begin{array}{lll}
\mathrm{U}_{\text {walls }} & = & \begin{array}{l}
\text { results of Section A.1 [should be } 0.400 \mathrm{~W} / \mathrm{m}^{2}{ }^{\circ} \mathrm{C} \\
\left.\left(0.0704 \mathrm{Btu} / \mathrm{h} \mathrm{ft}^{2}{ }^{\circ} \mathrm{F}\right)\right]
\end{array} \\
\mathrm{A}_{\text {walls }} & = & 65.5 \mathrm{~m}^{2}\left(705 \mathrm{ft}^{2}\right) \\
(\mathrm{UA})_{\text {walls }}= & \left(0.400 \mathrm{~W} / \mathrm{m}^{2}{ }^{\circ} \mathrm{C}\right)\left(65.5 \mathrm{~m}^{2}\right)=26.19 \mathrm{~W} /{ }^{\circ} \mathrm{C}\left(49.63 \mathrm{Btu} / \mathrm{h}{ }^{\circ} \mathrm{F}\right) \\
(\mathrm{UA})_{\text {window }}= & \dot{q} / 22.2^{\circ} \mathrm{C}-26.19 \mathrm{~W} /{ }^{\circ} \mathrm{C} \\
\mathrm{U}_{\text {window }} & = & (\mathrm{UA})_{\text {window }} /(\text { area of windows })=(\mathrm{UA})_{\text {window }} / 0.465 \mathrm{~m}^{2} \\
& {\left[(\mathrm{UA})_{\text {window }} / 5 \mathrm{ft}^{2}\right] .}
\end{array}
$$

This value of $U_{\text {window }}$ should be calculated for single, double, and triple glazings. For comparison, ASHRAE [2] values are given in Table A-7.

Table A-7. ASHRAE Glazing U-Values

\begin{tabular}{|l|c|c|}
\hline \multirow{2}{*}{\multicolumn{1}{c|}{ Glazing Type }} & \multicolumn{2}{|c|}{ U-Value } \\
\cline { 2 - 3 } & $\mathbf{( W / \mathbf { m } ^ { 2 } { } ^ { \circ } \mathbf { C } )}$ & $\left.\mathbf{( B t u / h} \mathbf{~ f t}^{2}{ }^{\circ} \mathbf{F}\right)$ \\
\hline Single & $5.91-6.25$ & $1.04-1.10$ \\
\hline Double & $2.78-3.69$ & $0.49-0.65$ \\
\hline Triple & $1.76-2.50$ & $0.31-0.44$ \\
\hline
\end{tabular}




\section{Notes on the Implementation of the Test}

Glazing materials have a variety of physical properties. The properties listed in the building description were chosen to be typical of normal window glass. If the code has a library containing several types of glazings, choose the one that corresponds to regular glass (as opposed to heat absorbing, reflective, or other types).

\section{A.5 Conservation Test}

\section{Mechanisms Tested}

The effects of changes in insulation levels on the mechanisms tested in Section A.1.

\section{Weather Type}

A

\section{Test Building}

A

\section{Test Building Description}

Same as in Section A.1 except that the test is run twice, first with a building UA of $10.55 \mathrm{~W} /{ }^{\circ} \mathrm{C}$ $\left(20 \mathrm{Btu} / \mathrm{h}{ }^{\circ} \mathrm{F}\right)$ and then with a building $\mathrm{UA}$ of $79.13 \mathrm{~W} /{ }^{\circ} \mathrm{C}\left(150 \mathrm{Btu} / \mathrm{h}{ }^{\circ} \mathrm{F}\right)$. For building $\mathrm{UA}=$ $10.55 \mathrm{~W} /{ }^{\circ} \mathrm{C}\left(20 \mathrm{Btu} / \mathrm{h}{ }^{\circ} \mathrm{F}\right)$, wall section is:

- Inside film coefficient $=5.6786 \mathrm{~W} / \mathrm{m}^{2}{ }^{\circ} \mathrm{C}\left(1 \mathrm{Btu} / \mathrm{h} \mathrm{ft}{ }^{\circ} \mathrm{F}\right)$

- $0.0127-\mathrm{m}\left(0.5\right.$-in) gypsum board $\left[\mathrm{k}=0.4327 \mathrm{~W} / \mathrm{m}{ }^{\circ} \mathrm{C}\left(0.25 \mathrm{Btu} / \mathrm{h} \mathrm{ft}{ }^{\circ} \mathrm{F}\right)\right.$,

$$
\begin{aligned}
& \rho=1249 \mathrm{~kg} / \mathrm{m}^{3}\left(78 \mathrm{lb} / \mathrm{ft}^{3}\right), \\
& \left.\mathrm{c}_{\mathrm{p}}=1.087 \mathrm{~kJ} / \mathrm{kg}{ }^{\circ} \mathrm{C}\left(0.26 \mathrm{Btu} / \mathrm{lb}{ }^{\circ} \mathrm{F}\right)\right]
\end{aligned}
$$

- Zero mass insulation with an R-value of $6.002 \mathrm{~m}^{2}{ }^{\circ} \mathrm{C} / \mathrm{W}\left(34.084 \mathrm{~h} \mathrm{ft}^{2}{ }^{\circ} \mathrm{F} / \mathrm{Btu}\right)$

- Outside film coefficient $=22.7 \mathrm{~W} / \mathrm{m}^{2}{ }^{\circ} \mathrm{C}\left(4 \mathrm{Btu} / \mathrm{h} \mathrm{ft}{ }^{2}{ }^{\circ} \mathrm{F}\right)$

For building $\mathrm{UA}=79.13 \mathrm{~W} /{ }^{\circ} \mathrm{C}\left(150 \mathrm{Btu} / \mathrm{h}{ }^{\circ} \mathrm{F}\right)$, wall section is:

- Inside film coefficient $=5.6786 \mathrm{~W} / \mathrm{m}^{2}{ }^{\circ} \mathrm{C}\left(1 \mathrm{Btu} / \mathrm{h} \mathrm{ft}{ }^{\circ} \mathrm{F}\right)$

- 0.0127 -m (0.5-in.) gypsum board $\left[\mathrm{k}=0.4327 \mathrm{~W} / \mathrm{m}^{\circ} \mathrm{C}\left(0.25 \mathrm{Btu} / \mathrm{h} \mathrm{ft}{ }^{\circ} \mathrm{F}\right)\right.$

$$
\begin{aligned}
& \rho=1249 \mathrm{~kg} / \mathrm{m}^{3}\left(78 \mathrm{lb} / \mathrm{ft}^{3}\right), \\
& \left.\mathrm{c}_{\mathrm{p}}=1.089 \mathrm{~kJ} / \mathrm{kg}{ }^{\circ} \mathrm{C}\left(0.26 \mathrm{Btu} / \mathrm{lb}{ }^{\circ} \mathrm{F}\right)\right]
\end{aligned}
$$

- Zero mass insulation with an R-value of $0.584 \mathrm{~m}^{2}{ }^{\circ} \mathrm{C} / \mathrm{W}\left(3.316 \mathrm{~h} \mathrm{ft}^{2}{ }^{\circ} \mathrm{F} / \mathrm{Btu}\right)$

- Outside film coefficient $=22.7 \mathrm{~W} / \mathrm{m}^{2}{ }^{\circ} \mathrm{C}\left(4 \mathrm{Btu} / \mathrm{h} \mathrm{ft}^{2}{ }^{\circ} \mathrm{F}\right)$

\section{Procedure}

Run the test twice, once for each insulation level. Run the $79.13 \mathrm{~W} /{ }^{\circ} \mathrm{C}\left(150 \mathrm{Btu} / \mathrm{h}{ }^{\circ} \mathrm{F}\right)$ building for January only. 


\section{Interpretation of Results}

\section{Steady-State Tests}

The same as in Section A.1, except that the steady-state building UA values should equal 10.55 $\mathrm{W} / \mathrm{Q}\left(20 \mathrm{Btu} / \mathrm{h}{ }^{\circ} \mathrm{F}\right)$ and $79.13 \mathrm{~W} /{ }^{\circ} \mathrm{C}\left(150 \mathrm{Btu} / \mathrm{h}{ }^{\circ} \mathrm{F}\right)$, respectively.

\section{Dynamic Tests}

For the $10.55 \mathrm{~W} /{ }^{\circ} \mathrm{C}\left(20 \mathrm{Btu} / \mathrm{h}{ }^{\circ} \mathrm{F}\right)$ building, this is the same as in Section A.1 except we extended the limits of $\tau$ so $0<\tau<20$, and computed $\tau=0$ at hour 10 of the dynamic test period.

$$
\mathrm{T}_{\mathrm{NA}}(\mathrm{t})=\exp \left[-\left(\mathrm{U}_{\mathrm{o}} / \mathrm{C}_{\mathrm{w}}\right) \mathrm{t}\right], 0<\mathrm{t} \leq 20 \mathrm{hr}
$$

Where,

$$
\begin{array}{ll}
\mathrm{U}_{\mathrm{o}} & =\left(0.5 \mathrm{R}_{\text {gyp }}+\mathrm{R}_{\text {ins }}+\mathrm{R}_{\text {off }}\right)^{-1} \\
\mathrm{R}_{\text {gyp }} & =0.0294 \mathrm{~m}^{2}{ }^{\circ} \mathrm{C} / \mathrm{W}\left(0.167 \mathrm{~h} \mathrm{ft}^{2}{ }^{\circ} \mathrm{F} / \mathrm{Btu}\right) \\
\mathrm{R}_{\text {ins }} & =6.002 \mathrm{~m}^{2}{ }^{\circ} \mathrm{C} / \mathrm{W}\left(34.084 \mathrm{~h} \mathrm{ft}^{2}{ }^{\circ} \mathrm{F} / \mathrm{Btu}\right) \\
\mathrm{R}_{\text {ofc }}= & 0.044 \mathrm{~W} / \mathrm{m}^{2}{ }^{\circ} \mathrm{C}\left(0.25 \mathrm{Btu} / \mathrm{h} \mathrm{ft}^{2}{ }^{\circ} \mathrm{F}\right) .
\end{array}
$$

Therefore,

$$
\begin{aligned}
\mathrm{U}_{\mathrm{o}} & =[0.5(0.0294)+6.002+0.044]^{-1} \mathrm{~W} / \mathrm{m}^{2}{ }^{\circ} \mathrm{C} \\
& =0.165 \mathrm{~W} / \mathrm{m}^{\circ} \mathrm{C} \\
\mathrm{C}_{\mathrm{w}} & =17,274 \mathrm{~J} / \mathrm{m}^{2}{ }^{\circ} \mathrm{C} .
\end{aligned}
$$

Therefore,

$$
\begin{aligned}
\mathrm{T}_{\mathrm{NA}}(\tau) & =\exp -\left[\left(0.165 \mathrm{~W} / \mathrm{m}^{2}{ }^{\circ} \mathrm{C}\right) /\left(17,274 \mathrm{~J} / \mathrm{m}^{2}{ }^{\circ} \mathrm{C}\right)\right] \tau \\
& =\exp -(0.000096 / \mathrm{s}) \tau=\exp -(0.0344 / \mathrm{h}) \tau /
\end{aligned}
$$

These values are presented in Table A-8 and Figure A-4.

For the $79.13 \mathrm{~W} /{ }^{\circ} \mathrm{C}\left(150 \mathrm{Btu} / \mathrm{h}{ }^{\circ} \mathrm{F}\right)$ building, do not run a dynamic test since the building changes temperature so quickly it is difficult to obtain meaningful results.

\section{Notes on Implementation of the Test}

See Section. A.1.

Table A-8. Normalized Highly Insulated Low-Mass Temperature Decay

\begin{tabular}{|c|c|}
\hline $\mathbf{t}(\mathbf{h r})$ & TNA(T) \\
\hline 0 & 1.0 \\
\hline 2 & 0.934 \\
\hline 4 & 0.872 \\
\hline 6 & 0.814 \\
\hline 8 & 0.760 \\
\hline 10 & 0.709 \\
\hline 12 & 0.662 \\
\hline 14 & 0.618 \\
\hline 16 & 0.577 \\
\hline 18 & 0.539 \\
\hline 20 & 0.503 \\
\hline
\end{tabular}




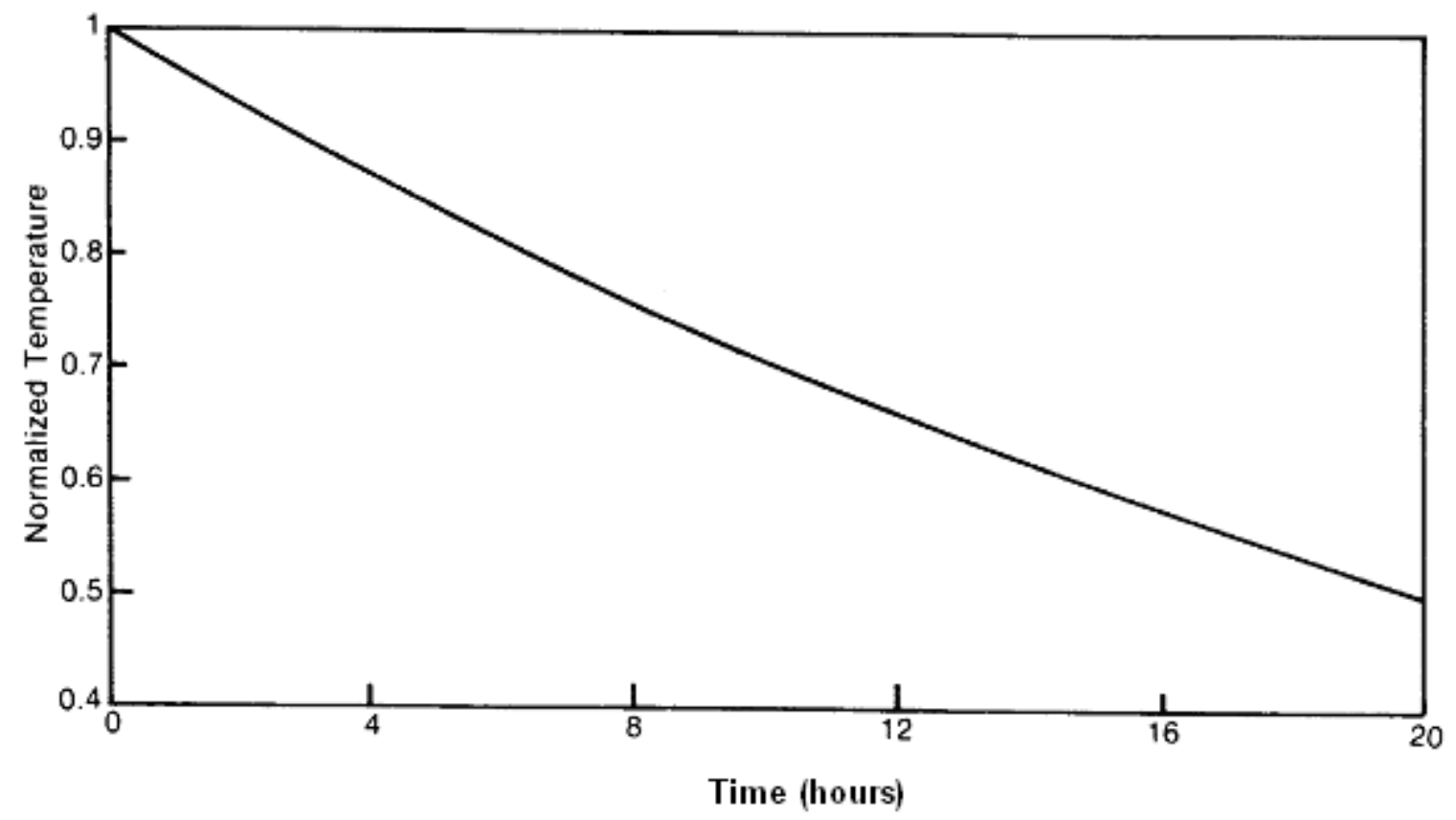

Figure A-4. Conservation test solution

\section{A.6 Infiltration Test}

\section{Mechanisms Tested}

Heat transfer rates are associated with the infiltration rate. This test is a check on the assumptions involving the infiltration mechanisms in a code.

\section{Weather Type}

A (January)

\section{Test Building}

A

\section{Test Building Description}

Same as in Sec. A.1 except that the test is run six times with air infiltration rates of $0.5,1,1.5,2$, 3 , and 5 air changes per hour $(\mathrm{ACH})$. These rates expressed in equivalent units are listed in Table A-9. The volume of the test building is $33.98 \mathrm{~m}^{3}\left(1200 \mathrm{ft}^{3}\right)$.

\section{Procedure}

Run this test six times, once for each infiltration level. The $0 \mathrm{ACH}$ run is same as in Section A.1. 
Table A-9. Air Change Rate Conversion Factors

\begin{tabular}{|c|c|c|c|}
\hline $\mathbf{A C H}$ & $\begin{array}{c}\mathbf{m}^{3} / \mathbf{h} \\
\left(\mathbf{f t}^{3} / \mathbf{h}\right)\end{array}$ & $\begin{array}{c}\mathbf{m}^{3} / \mathbf{m i n} \\
\left(\mathrm{ft}^{3} / \mathbf{m i n}\right)\end{array}$ & $\begin{array}{c}\left(\mathbf{m}^{3} / \mathbf{m i n}\right) / \mathbf{m}^{2} \mathbf{a} \\
{\left[\left(\mathbf{f t}^{3} / \mathbf{m i n}^{2} / \mathrm{ft}^{2}\right]^{\mathbf{~}}\right.}\end{array}$ \\
\hline 0 & 0 & 0 & 0 \\
\hline 0.5 & $16.99(600)$ & $0.283(10)$ & $0.0203(0.067)$ \\
\hline 1 & $33.98(1200)$ & $0.566(20)$ & $0.0406(0.133)$ \\
\hline 1.5 & $50.97(1800)$ & $0.850(30)$ & $0.0610(0.200)$ \\
\hline 2 & $67.96(2400)$ & $1.133(40)$ & $0.0813(0.267)$ \\
\hline 3 & $101.94(3600)$ & $1.699(60)$ & $0.122(0.400)$ \\
\hline 5 & $169.90(6000)$ & $2.832(100)$ & $0.203(0.667)$ \\
\hline
\end{tabular}

${ }^{a}$ This is the air change rate in $\mathrm{m}^{3} / \mathrm{min}$ divided by the floor area.

${ }^{\mathrm{b}}$ This is the air change rate in $\mathrm{ft}^{3} / \mathrm{min}$ divided by the floor area.

\section{Interpretation of Results}

The steady-state auxiliary heating load is read from the output of each run. These loads can be tabulated and the effective heat transfer coefficient for each infiltration rate can be calculated in the following manner:

$$
\dot{q}_{\text {total }}=\dot{q}_{\text {shell }}+\dot{q}_{\text {infiltration }}
$$

or

$$
\dot{q}_{\text {infiltration }}=\dot{q}_{\text {total }}-\dot{q}_{\text {shell }}
$$

Where,

$$
\begin{array}{lll}
\dot{q}_{\text {total }} & = & \text { total building heating load } \\
\dot{q}_{\text {shell }} & = & \begin{array}{l}
\text { building load from conduction losses through the building } \\
\text { shell }
\end{array} \\
\dot{q}_{\text {infiltration }} & = & \text { building load from infiltration losses }
\end{array}
$$

but:

$$
\dot{q}_{\text {shell }}=\quad \begin{aligned}
& \dot{q}_{\text {shell }} \text { from Section A.1, which should equal } 586.1 \mathrm{~W}(2000) \\
& \text { Btu/h) }
\end{aligned}
$$

Therefore,

$$
\dot{q}_{\text {infiltration }} \quad=\quad \dot{q}_{\text {total }}-586.1 \mathrm{~W}(2000 \mathrm{Btu} / \mathrm{h}) .
$$

Also,

$$
\begin{aligned}
\left(\mathrm{UA}_{\mathrm{eff}}\right)_{\text {infiltration }}= & \dot{q}_{\text {infiltration }} /\left(\mathrm{T}_{\text {inside }}-\mathrm{T}_{\text {outside }}\right) \\
& =\quad \dot{q}_{\text {infiltration }} / 22.2^{\circ} \mathrm{C}\left(40^{\circ} \mathrm{F}\right)
\end{aligned}
$$

Where

$$
\begin{array}{ll}
\left(\mathrm{UA}_{\mathrm{eff}}\right)_{\text {infiltration }}=\quad \begin{array}{l}
\text { the effective heat transfer coefficient resulting from } \\
\text { infiltration. }
\end{array}
\end{array}
$$

These values can be compared to the values derived from the formula,

$$
(\mathrm{UA})_{\mathrm{eff}}=\left(1.207 \mathrm{~kJ} / \mathrm{m}^{3}{ }^{\circ} \mathrm{C}\right)(\mathrm{V})(\mathrm{ACH})=\left(0.018 \mathrm{Btu} / \mathrm{ft}^{3}{ }^{\circ} \mathrm{F}\right)(\mathrm{V})(\mathrm{ACH})
$$


where

$$
\begin{array}{lll}
\mathrm{V} & = & \text { building volume }=33.98 \mathrm{~m}^{3}\left(1200 \mathrm{ft}^{3}\right) \\
\mathrm{ACH} & = & \text { infiltration rate in air changes/hour } \\
1.207 \mathrm{kJm}^{3}{ }^{\circ} \mathrm{C} & = & \left(0.018 \mathrm{Btu} / \mathrm{ft}{ }^{\circ} \mathrm{F}\right) \\
& = & \text { volumetric heat capacity of dry air at sea level pressure and } \\
& 21.1^{\circ} \mathrm{C}\left(70^{\circ} \mathrm{F}\right)
\end{array}
$$

These values are given in Table A-10.

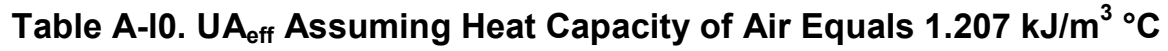

\begin{tabular}{|c|c|c|}
\hline $\begin{array}{c}\text { Infiltration Rate } \\
\text { (ACH) }\end{array}$ & \multicolumn{2}{|c|}{ UA $_{\text {eff }}$} \\
\hline & $\mathbf{W} /{ }^{\circ} \mathbf{C}$ & (Btu/h \\
& \\
& & $\mathbf{F})$ \\
\hline 0 & 0 & 0 \\
\hline 0.5 & 5.7 & 10.8 \\
\hline 1 & 11.4 & 21.6 \\
\hline 1.5 & 17.1 & 32.4 \\
\hline 2 & 22.8 & 43.2 \\
\hline 3 & 34.2 & 64.8 \\
\hline 5 & 57.0 & 108.0 \\
\hline
\end{tabular}

Different algorithms for infiltration could produce results that differ from these values. The differences could be caused by algorithms that account for the effect of attitude, air pressure, and air moisture content. These effects should be reduced by using the values in weather file A. Large differences between the results from the code and these values should be investigated.

\section{Notes on Implementation of the Test}

These tests assume a constant infiltration rate, unaffected by wind or differences between the internal and external temperatures. Care must be taken to ensure that these conditions are satisfied by either the input files or temporary modification to the code.

\section{A.7 Solar, Charging, and Window Transmissivity Test}

\section{Mechanism Tested}

Window transmissivity for diffuse radiation

The thermal response of the thermal mass to solar radiation

\section{Weather Type}

\section{B}

\section{Test Building}

B

\section{Test Building Description}

- No infiltration

- No ground coupling 
- Zero external absorptivity/emissivity

- Internal absorptivity/emissivity $=1.0$ (solar radiation)

- If possible, internal absorptivity/emissivity $=0$ (longwave radiation)

- Single zone

- Building is at sea level

Wall characteristics:

- Inside film coefficient $5.6786 \mathrm{~W} / \mathrm{m}^{2}{ }^{\circ} \mathrm{C}\left(1 \mathrm{Btu} / \mathrm{h} \mathrm{ft}^{2}{ }^{\circ} \mathrm{F}\right)$

- $0.0127-\mathrm{m}\left(0.5\right.$-in.) gypsum board $\left[\mathrm{k}=0.4327 \mathrm{~W} / \mathrm{m}^{\circ} \mathrm{C}\left(0.25 \mathrm{Btu} / \mathrm{h} \mathrm{ft}{ }^{\circ} \mathrm{F}\right)\right.$,

$$
\begin{aligned}
& \rho=1249 \mathrm{~kg} / \mathrm{m}^{3}\left(78 \mathrm{lb} / \mathrm{ft}^{3}\right), \\
& \left.\mathrm{Cp}=1.089 \mathrm{~kJ} / \mathrm{kg}{ }^{\circ} \mathrm{C}\left(0.26 \mathrm{Btu} / \mathrm{lb}{ }^{\circ} \mathrm{F}\right)\right]
\end{aligned}
$$

- Enough pure resistance (zero mass insulation) to decouple the gypsum board from the outside temperature. If input requires a conductivity, set it equal to 0 ; if an R-value is required, set it to 1 million or as large a number as the code will accept.

- Wall area $=65.5 \mathrm{~m}^{2}\left(705 \mathrm{ft}^{2}\right)$

Note: This wall section applies to south, east, north, and west walls and ceiling.

Window characteristics:

- Window area $=0.465 \mathrm{~m}^{2}\left(705 \mathrm{ft}^{2}\right)$

- Vertical

- Normal window glass

- Pane thickness $=3.18 \mathrm{~mm}(0.125 \mathrm{in}$. $)$

- $\quad$ Extinction coefficient $=0.197 / \mathrm{cm}(0.5 / \mathrm{in}$. $)$

- Index of refraction $=1.526$

- Air-gap $=6.35 \mathrm{~mm}(0.25 \mathrm{in}$.)

- Diffuse angle of incidence $=60 \mathrm{deg}$

- Double glazed

- Zero window conductivity if possible, or as low as the code will allow

- Cooling set point $=26.67^{\circ} \mathrm{C}\left(80^{\circ} \mathrm{F}\right)$ for first part, or very high $\left[>93.3^{\circ} \mathrm{C}\left(200^{\circ} \mathrm{F}\right)\right]$ for second part of this test

- Zero ground reflectivity

\section{Procedure}

Run the code with weather type B for January and February and a cooling set point of $26.67^{\circ} \mathrm{C}$ $\left(80^{\circ} \mathrm{F}\right)$. The results from this run will be used to calculate the effective diffuse transmissivity of 
the glazings. Then run the test again with the cooling set point over $93.3^{\circ} \mathrm{C}\left(200^{\circ} \mathrm{F}\right)$. This run will be used to determine the effect of solar radiation on the thermal mass in the building.

\section{Interpretation of Results}

The diffuse transmissivity of the glazing can be determined from the first run through this test; the one with the cooling set point of $26.7^{\circ} \mathrm{C}\left(80^{\circ} \mathrm{F}\right)$. The diffuse transmissivity is the ratio of the transmitted radiation divided by the radiant energy incident on the exterior of the glazing, which is all diffuse. The incident radiation can be read as an output in many codes, or can be calculated as:

$$
G_{V}^{D}=0.5 G_{H}^{D}+0.5 \rho_{\mathrm{G}}(\mathrm{GDH})
$$

Where,

$$
\begin{array}{rll}
G_{V}^{D} & = & \text { incident diffuse radiation flux on a vertical surface } \\
G_{H}^{D} & = & \text { global diffuse radiation flux } \\
\rho_{\mathrm{G}} & = & \text { ground reflectivity. }
\end{array}
$$

Notes: If $\rho_{\mathrm{gr}}=0$, then $G_{V}^{D}=(0.5) G_{H}^{D}$. The 0.5 in the above equation is the radiation view factor between the sky and a vertical surface and between the ground and a vertical surface.

The transmitted diffuse radiation flux $G_{V}^{\text {Tran }}$ can be read as the building cooling load in February after steady-state conditions have been reached. Thus, the glazing transmissivity is

$$
\tau_{G}=G_{V}^{\operatorname{Tran}} / G_{V}^{D}
$$

Note that this may include thermal transfer of energy absorbed in the glass if relevant mechanisms have not been eliminated. The temperature response of the internal mass due to the transmitted diffuse solar radiation can be determined by the run in which the cooling set point is at or above $93^{\circ} \mathrm{C}\left(200^{\circ} \mathrm{F}\right)$. The incoming diffuse radiation is assumed to hit all interior building surfaces with equal flux, which is:

$$
Q=G_{V}^{\text {Tran }} A_{\text {Window }} / A_{\text {Wall }}
$$

Where,

$$
\begin{array}{lll}
\mathrm{A}_{\text {window }} & = & \text { window area }=0.465 \mathrm{~m}^{2}\left(5 \mathrm{ft}^{2}\right) \\
\mathrm{A}_{\text {wall }} & = & \text { internal wall area }=65.5 \mathrm{~m}^{2}\left(705 \mathrm{ft}^{2}\right) .
\end{array}
$$

The interior air temperature can be read from the output of the code, and this temperature will exactly equal the interior wall surface temperature if there are no heat flow paths between the air and the outside except for through the massive walls. For comparison to the analytical solution, this air temperature must be normalized to the incoming radiant flux, such that equation TNR can be compared with the results from the analytical solution shown in Table A-11 and Figure A-5. 
Table A-11. Normalized Low-Mass Solar-Driven Temperature Rise

\begin{tabular}{|c|c|c|}
\hline $\mathbf{t}(\mathbf{h r})$ & $\mathbf{m}^{\mathbf{2}}{ }^{\circ} \mathbf{C} \mathbf{W}$ & $\mathbf{h ~ f t}^{2}{ }^{\circ} \mathbf{F} / \mathbf{B t u}$ \\
\hline 0 & 0 & 0 \\
\hline 1 & 0.218 & 1.238 \\
\hline 2 & 0.426 & 2.421 \\
\hline 3 & 0.635 & 3.603 \\
\hline 4 & 0.843 & 4.786 \\
\hline 5 & 1.051 & 5.968 \\
\hline 6 & 1.259 & 7.151 \\
\hline 7 & 1.468 & 8.333 \\
\hline 8 & 1.676 & 9.516 \\
\hline 9 & 1.884 & 10.698 \\
\hline 10 & 2.092 & 11.881 \\
\hline
\end{tabular}

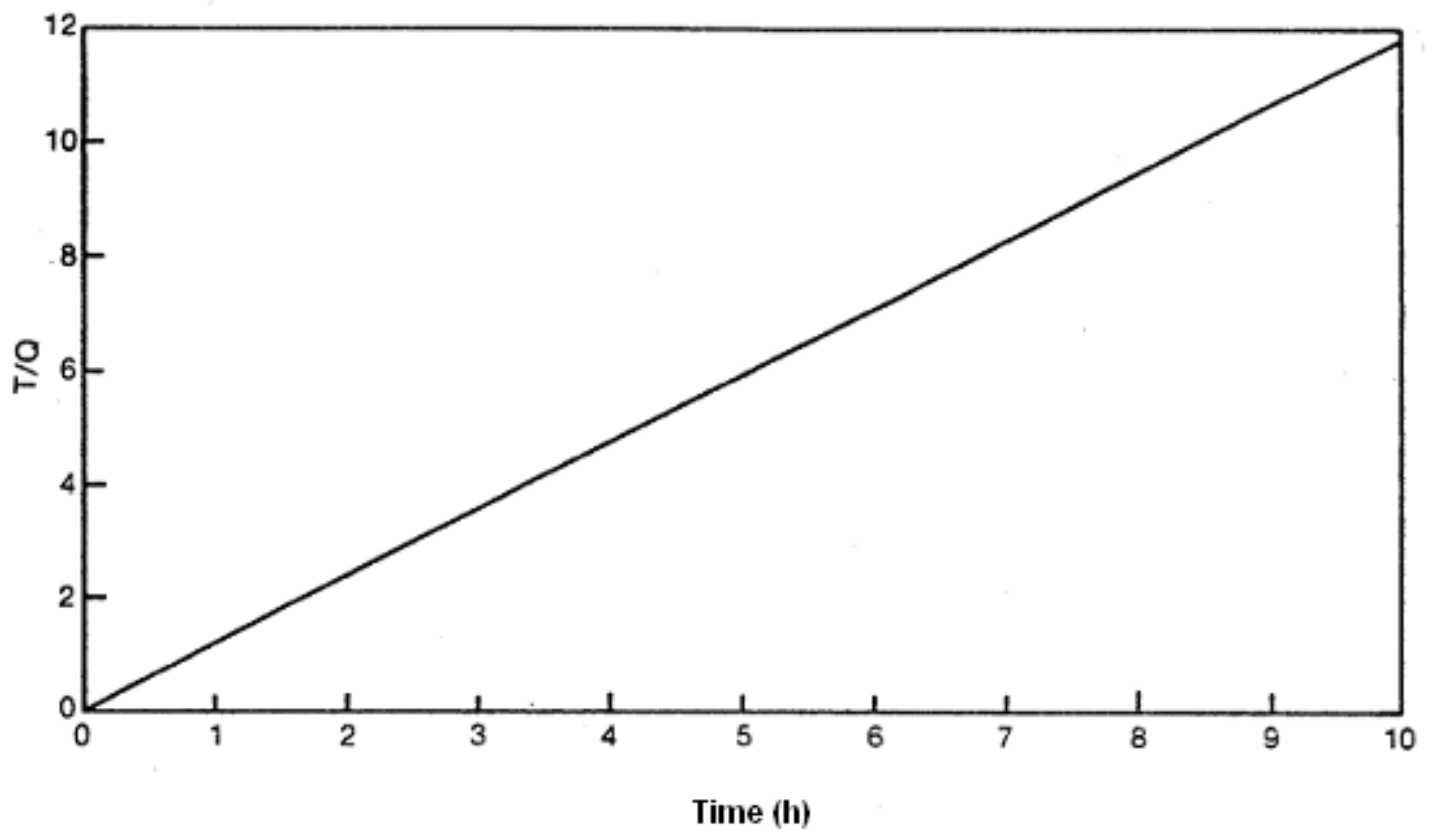

Figure A-5. Normalized low-mass solar driven temperature rise versus time

\section{Notes on Implementation of the Test}

This test is the basis of the remaining tests, and must be done accurately. Mechanisms that do not correspond to the initial and boundary conditions of the analytical solution must be eliminated or modified. These mechanisms include the zero window conductivity and infinite resistance (or zero conductance) between the mass and the outside. Mechanisms causing the transfer of absorbed energy into the room should also be eliminated. The cooling load and, if possible, solar radiation transmitted through the glazing must be output to enable calibration of the results against the analytical solution.

\section{A.8 Glazing Transmissivity Test}

\section{Mechanism Tested}

The effect of changes in the number of glazings on window transmissivity for diffuse radiation. This test is a check on a code's inherent assumptions about glazing transmissivity. 
Weather Type

$\mathrm{B}$

Test Building

B

\section{Test Building Description}

Same as in Section A.7 except run the test twice, once with single glazing and once with triple glazing.

\section{Procedure}

Run the test twice, once for each glazing configuration with the cooling set point at $26.67^{\circ} \mathrm{C}$ $\left(80^{\circ} \mathrm{F}\right)$. Rerun each with the higher cooling set point, as in Section A.7.

Table A-12. Glazing Diffuse Transmissivity

\begin{tabular}{|c|c|}
\hline Number of Glazings & Diffuse Transmissivity \\
\hline 1 & 0.78 \\
\hline 2 & 0.64 \\
\hline 3 & 0.54 \\
\hline
\end{tabular}

\section{Interpretation of Results}

Use the same procedure as in Section A.7 to determine the diffuse transmissivity for the single- and triple-glazed cases.. The results for the three glazing types can be compared to the values in Table A-12. These theoretical values are derived for glazing with the properties listed in the above Test Building Description by the methods described in Carlslaw and Jaeger.

\section{A.9 Mid-Mass Charge}

\section{Mechanism Tested}

The effect of changes in thermal mass on the temperature response of the mass to solar radiation.

\section{Weather Type}

$\mathrm{B}$

\section{Test Building}

B

\section{Test Building Description}

Same as in Section A.7 except

Wall characteristics:

- Inside film coefficient $=5.6786 \mathrm{~W} / \mathrm{m}^{2}{ }^{\circ} \mathrm{C}\left(1 \mathrm{Btu} / \mathrm{h} \mathrm{ft}{ }^{\circ} \mathrm{F}\right)$

- $0.1016-\mathrm{m}(0.25$-in. $)$ concrete $\left[\mathrm{k}=0.935 \mathrm{~W} / \mathrm{m}{ }^{\circ} \mathrm{C}\left(0.54 \mathrm{Btu} / \mathrm{h} \mathrm{ft}{ }^{\circ} \mathrm{F}\right)\right.$

$\left.\rho=144 \mathrm{lb} / \mathrm{ft}^{3}\left(2307 \mathrm{~kg} / \mathrm{m}^{3}\right), \mathrm{c}_{\mathrm{p}}=0.67 \mathrm{~kJ} / \mathrm{kg}{ }^{\circ} \mathrm{C}\left(0.16 \mathrm{Btu} / \mathrm{lb}{ }^{\circ} \mathrm{F}\right)\right]$

- Same as in Section A.7. 


\section{Procedure}

Same as in Section A.7. 
Table A-13. Normalized Mid-Mass Solar-Driven Temperature Rise

\begin{tabular}{|c|c|c|}
\hline $\mathbf{t}(\mathbf{h r})$ & $\mathbf{m}^{2}{ }^{\circ} \mathbf{C} / \mathbf{W}$ & $\mathbf{h ~ f t}^{2}{ }^{\circ} \mathbf{F} / \mathbf{B t u}$ \\
\hline 0 & 0 & 0 \\
\hline 4 & 0.128 & 0.7266 \\
\hline 8 & 0.220 & 1.2474 \\
\hline 12 & 0.311 & 1.7683 \\
\hline 16 & -0.403 & 2.2891 \\
\hline 20 & 0.495 & 2.8099 \\
\hline 24 & 0.587 & 3.3308 \\
\hline 28 & 0.678 & 3.8516 \\
\hline 32 & 0.770 & 4.3724 \\
\hline 36 & 0.861 & 4.8933 \\
\hline 40 & 0.954 & 5.4141 \\
\hline
\end{tabular}

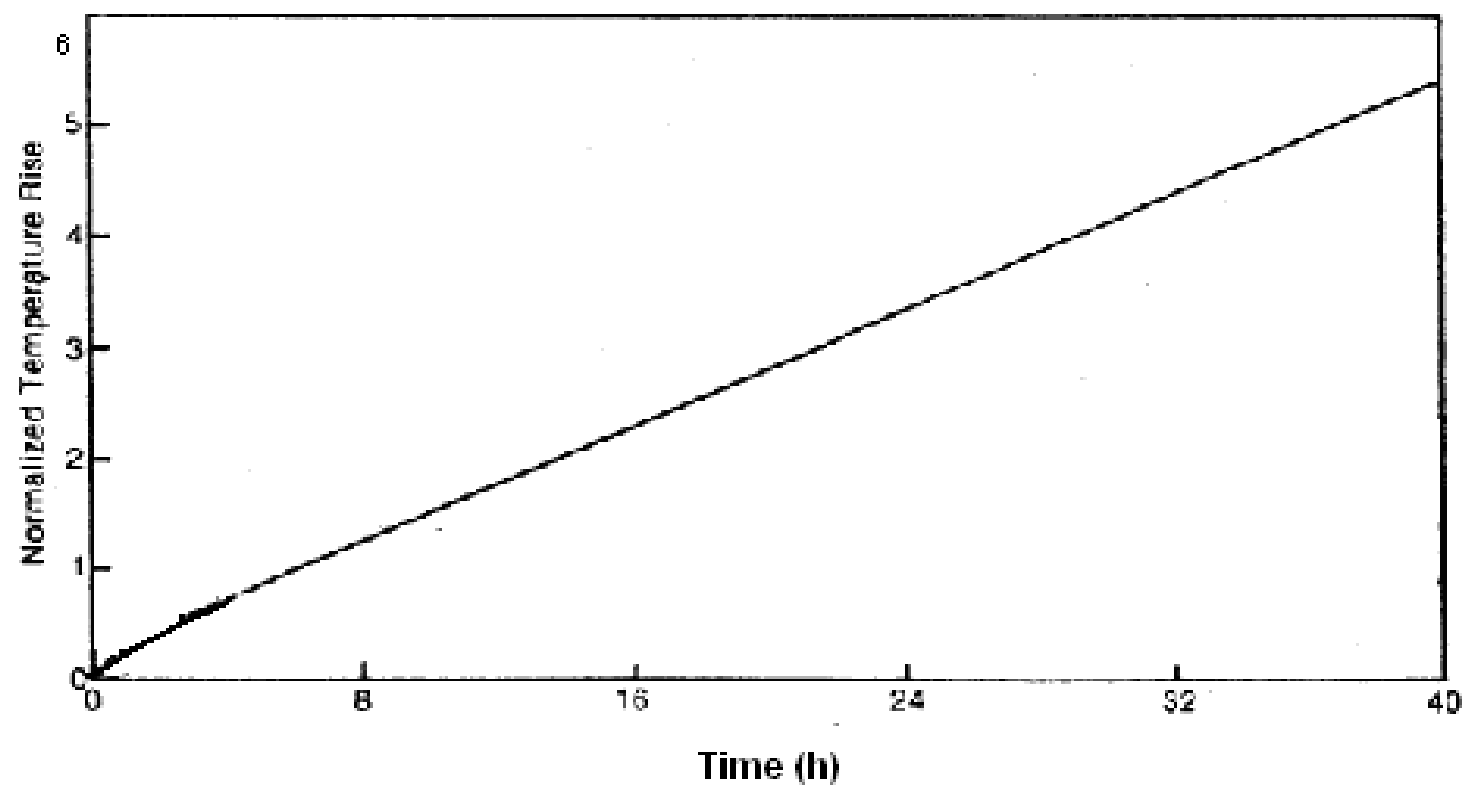

Figure A-6. Normalized mid-mass solar driven temperature rise versus time

\section{Interpretation of Results}

The transmissivity of the glazing can be calculated as in Section A.7, and the results should be identical. The normalized temperature response of the internal mass can be calculated as in Section A.7 and compared to Table A-13.

\section{Notes on Implementation of the Test}

See Sec. A.7.

\section{A.10 High-Mass Charge Test}

\section{Mechanism Tested}

The effect of changes in thermal mass on the temperature response of the mass to solar radiation. 


\section{Weather Type}

$\mathrm{B}$

\section{Test Building}

B

\section{Test Building Description}

Same as Section A.7, except

Wall characteristics:

$$
\begin{aligned}
& 0.1178-\mathrm{m}(7-\mathrm{ft} .) \text { concrete }\left[\mathrm{k} \bullet 0.935 \mathrm{~W} / \mathrm{m}^{\circ} \mathrm{C}\left(0.54 \mathrm{Btu} / \mathrm{h} \mathrm{ft}{ }^{\circ} \mathrm{F}\right),\right. \\
& \left.\rho=2307 \mathrm{~kg} / \mathrm{m}^{3}\left(144 \mathrm{lb} / \mathrm{ft}^{3}\right), \mathrm{Cp} \cdot 0.67 \mathrm{~kJ} / \mathrm{kg}{ }^{\circ} \mathrm{C}\left(0.16 \mathrm{Btu} / \mathrm{lb}{ }^{\circ} \mathrm{F}\right)\right]
\end{aligned}
$$

\section{Procedure}

Same as in Section A.7.

\section{Interpretation of Results}

The transmissivity of the glazing can be calculated as in Section A.7, and the results should be identical. The normalized temperature response of the internal mass can be calculated as in Section A.7, and compared to Table A-14 and Figure A.7.

Table A-14. Normalized High-Mass Solar-Driven Temperature Rise

\begin{tabular}{|c|c|c|}
\hline $\mathbf{t ~ ( h r ) ~}$ & $\mathbf{m}^{\mathbf{2}}{ }^{\circ} \mathbf{C} / \mathbf{W}$ & $\mathbf{h ~ f t}^{\mathbf{}}{ }^{\circ} \mathbf{F} / \mathbf{B t u}$ \\
\hline 0 & 0 & 0 \\
\hline 1 & 0.057 & 0.323 \\
\hline 2 & 0.080 & 0.453 \\
\hline 3 & 0.098 & 0.555 \\
\hline 4 & 0.113 & 0.643 \\
\hline 5 & 0.128 & 0.725 \\
\hline 10 & 0.194 & 1.104 \\
\hline 20 & 0.325 & 1.848 \\
\hline 30 & 0.457 & 2.592 \\
\hline 40 & 0.588 & 3.336 \\
\hline 50 & 0.719 & 4.081 \\
\hline 60 & 0.850 & 4.825 \\
\hline 70 & 0.981 & 5.569 \\
\hline 80 & 1.112 & 6.313 \\
\hline 90 & 1.243 & 7.057 \\
\hline 100 & 1.374 & 7.801 \\
\hline
\end{tabular}

Notes on Implementation of the Test

See Section A.7. 


\section{Appendix B}

Table B-1 gives estimates of clear day temperature elevation above ambient and temperature swing t treating the cell zones as if they were independent. Impact of various infiltration rates on the zone load coefficients is shown in Table B-2. Change in glazing air to air conductance is detailed in Table B-3.

Table B-1. Temperature Estimates

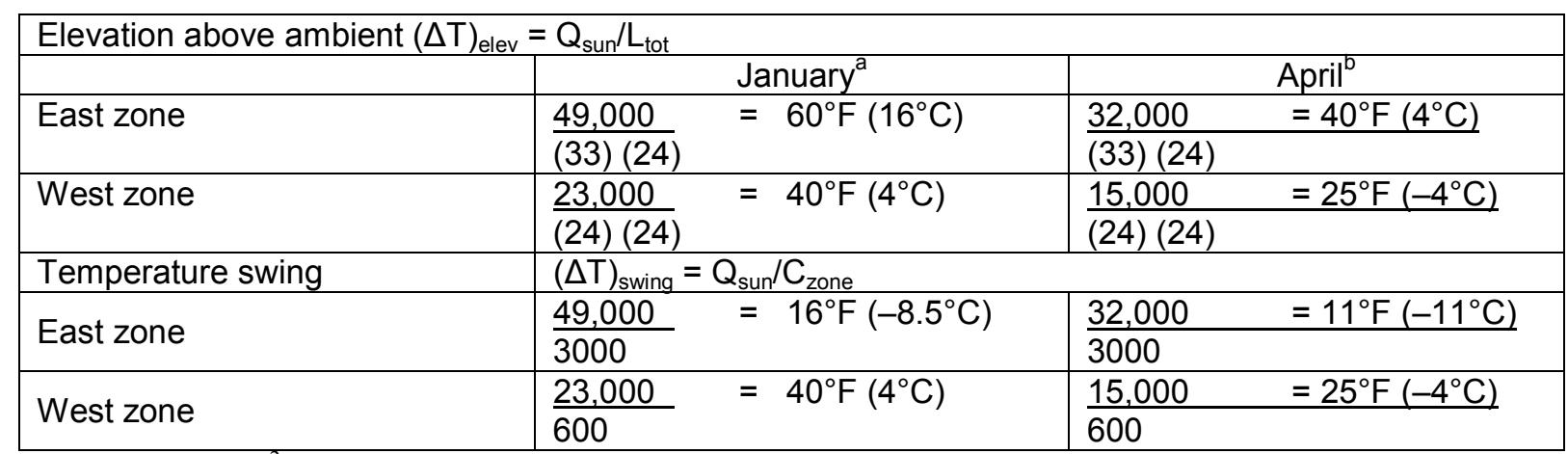

${ }^{\mathrm{a}} \mathrm{Q}_{\text {sun }}=1390 \mathrm{Btu} / \mathrm{ft}^{2}$-glazing-day

$\mathrm{b}_{\text {sun }}=900 \mathrm{Btu} / \mathrm{ft}^{2}$-glazing-day

Table B-2. Infiltration Loads

\begin{tabular}{|c|c|c|c|c|}
\hline \multirow{2}{*}{ Infiltration } & \multicolumn{2}{|c|}{ East Zone } & \multicolumn{2}{c|}{ West Zone } \\
\cline { 2 - 5 } & $(\mathbf{U A})_{\text {inf }}$ & $(\mathbf{U A})_{\text {inf }} /(\mathbf{U A})$ tot, $\%$ & $(\mathbf{U A})_{\text {inf }}$ & $(\mathbf{U A})_{\text {inf }} /(\mathbf{U A})_{\text {tot }}$ \\
\hline $0.07 \mathrm{ACH}$ & 0.6 & $2 \%$ & 0.6 & $2.5 \%$ \\
\hline $0.4 \mathrm{ACH}$ & 3.6 & $11 \%$ & 3.6 & $15 \%$ \\
\hline
\end{tabular}

Note: $(\mathrm{UA})_{\mathrm{inf}}=(\mathrm{pc}) \dot{V}$

Table B-3. Glazing Conductance

\begin{tabular}{|c|c|}
\hline $\begin{array}{c}\text { Wind Velocity } \\
\mathbf{m} / \mathbf{s}(\mathbf{m p h})\end{array}$ & $\begin{array}{c}\text { Glazing Conductance } \\
\left(\mathbf{w} / \mathbf{m}^{\circ} \mathbf{C}\left(\mathbf{B t u}_{\mathbf{f t}}{ }^{\circ} \mathbf{F}\right)\right.\end{array}$ \\
\hline $0(0)$ & $2.27(0.40)$ \\
\hline $6.7(15)$ & $2.78(0.49)$ \\
\hline $13.4(30)$ & $2.90(0.51)$ \\
\hline
\end{tabular}




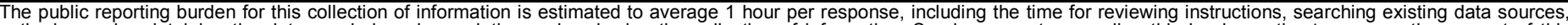

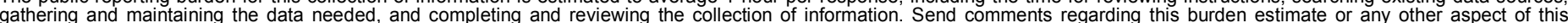

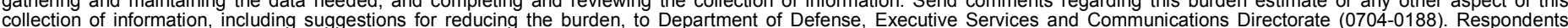

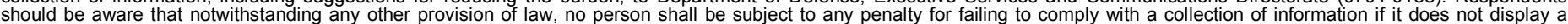

should be aware that notwithstanding

PLEASE DO NOT RETURN YOUR FORM TO THE ABOVE ORGANIZATION.

\begin{tabular}{l|l|l|l} 
1. REPORT DATE $(D D-M M-Y Y Y Y)$ & 2. REPORT TYPE & 3. DATES COVERED (FrOm - TO)
\end{tabular}

April 2008

Technical Report

4. TITLE AND SUBTITLE

A Methodology for Validating Building Energy Analysis Simulations

5a. CONTRACT NUMBER

DE-AC36-99-G010337

5b. GRANT NUMBER

5c. PROGRAM ELEMENT NUMBER

6. AUTHOR(S)

R. Judkoff, D. Wortman, B. O'Doherty, and J. Burch

5d. PROJECT NUMBER

NREL/TP-550-42059

5e. TASK NUMBER

54004000

5f. WORK UNIT NUMBER
7. PERFORMING ORGANIZATION NAME(S) AND ADDRESS(ES)

National Renewable Energy Laboratory

1617 Cole Blvd.

Golden, CO 80401-3393
8. PERFORMING ORGANIZATION REPORT NUMBER

NREL/TP-550-42059

9. SPONSORING/MONITORING AGENCY NAME(S) AND ADDRESS(ES)

10. SPONSOR/MONITOR'S ACRONYM(S) NREL

11. SPONSORING/MONITORING AGENCY REPORT NUMBER

12. DISTRIBUTION AVAILABILITY STATEMENT

National Technical Information Service

U.S. Department of Commerce

5285 Port Royal Road

Springfield, VA 22161

13. SUPPLEMENTARY NOTES

14. ABSTRACT (Maximum 200 Words)

The objective of this report was to develop a validation methodology for building energy analysis simulations, collect high-quality, unambiguous empirical data for validation, and apply the validation methodology to the DOE-2.1, BLAST-2MRT, BLAST-3.0, DEROB-3, DEROB-4, and SUNCAT 2.4 computer programs. This report covers background information, literature survey, validation methodology, comparative studies, analytical verification, empirical validation, comparative evaluation of codes, and conclusions.

15. SUBJECT TERMS

building energy analysis simulation; DOE-2.1; BLAST; BLAST; DEROB; SUNCAT

\begin{tabular}{l}
\hline \multicolumn{3}{|c|}{ 16. SECURITY CLASSIFICATION OF: } \\
\hline \begin{tabular}{l|l|l|} 
a. REPORT & b. ABSTRACT & c. THIS PAGE \\
Unclassified & Unclassified & Unclassified \\
& & \\
\end{tabular} \\
\hline
\end{tabular}

\begin{tabular}{|c|c|}
\hline $\begin{array}{l}\text { 17. LIMITATION } \\
\text { OF ABSTRACT }\end{array}$ & $\begin{array}{l}\text { 18. NUMBER } \\
\text { OF PAGES }\end{array}$ \\
\hline UL & \\
\hline
\end{tabular}

19a. NAME OF RESPONSIBLE PERSON

19b. TELEPHONE NUMBER (Include area code) 